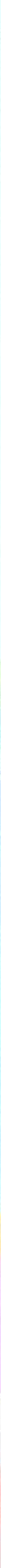




\section{-}

$x^{2}-12 x^{2}$ (2)

G

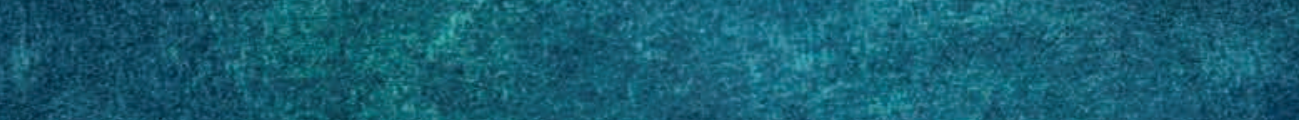
(15)

(1)

5.

3.7.

3.5. (1)

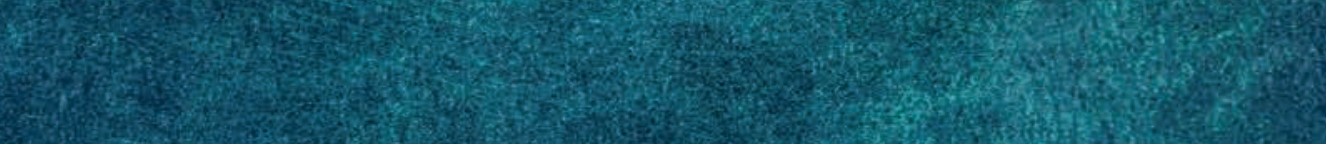

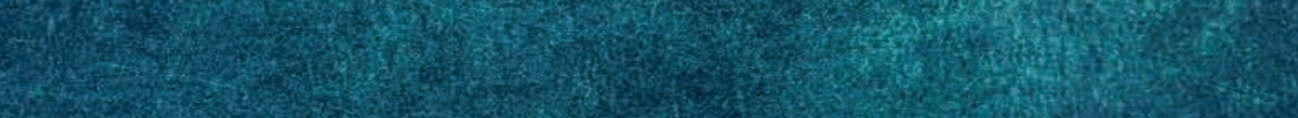

3.7.3.

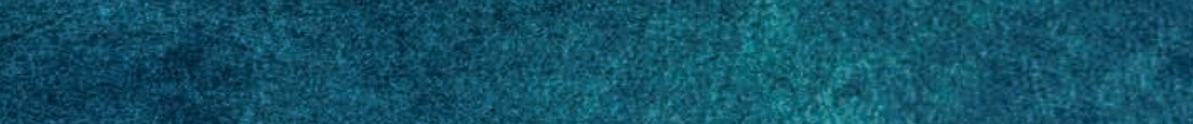

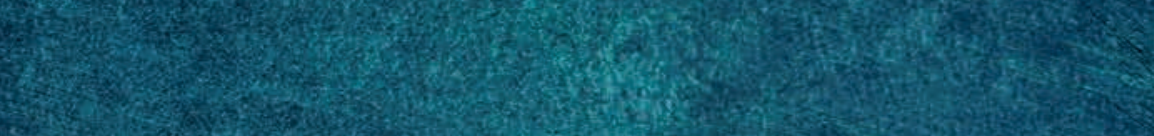

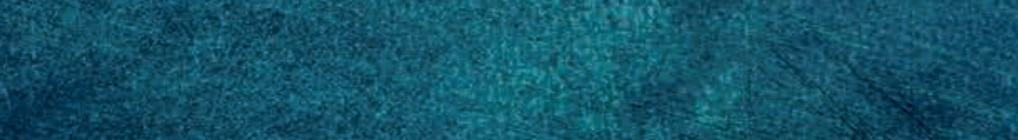




\title{
EFFICIENT MODELLING OF OFFSHORE PIPE-LAYING
}

\author{
Frans H. de Vries
}


Samenstelling van de promotiecommissie:

voorzitter en secretaris:

Prof.dr.ir. H.F.J.M. Koopman Universiteit Twente

promotoren:

Prof.dr.ir. A.H. van den Boogaard Universiteit Twente

Dr.ir. H.J.M Geijselaers Universiteit Twente

leden:

Prof.dr.ir. E.H. van Brummelen Technische Universiteit Eindhoven

Prof dr. A.V. Metrikine Technische Universiteit Delft

Dr. N.S. Ermolaeva Allseas

Prof.dr.ir. D.M. Brouwer Universiteit Twente

Prof.dr. S. Misra Universiteit Twente

Cover design \& illustrations: Ilse Schrauwers, isontwerp.nl

Printed by: Gildeprint

ISBN: 978-90-365-5030-7

DOI: $10.3990 / 1.9789036550307$

Funding information: The research leading to this thesis was financially supported by Allseas, which is gratefully acknowledged.

Copyright (C) 2020 by F.H. de Vries, Enschede, The Netherlands. All rights reserved. No parts of this thesis may be reproduced, stored in a retrieval system or transmitted in any form or by any means without permission of the author. Alle rechten voorbehouden. Niets uit deze uitgave mag worden vermenigvuldigd, in enige vorm of op enige wijze, zonder voorafgaande schriftelijke toestemming van de auteur. 


\title{
EFFICIENT MODELLING OF OFFSHORE PIPE-LAYING
}

\section{PROEFSCHRIFT}

\author{
ter verkrijging van \\ de graad van doctor aan de Universiteit Twente, \\ op gezag van de rector magnificus, \\ prof.dr. T.T.M. Palstra, \\ volgens besluit van het College voor Promoties \\ in het openbaar te verdedigen \\ op donderdag 17 september 2020 om 14:45 uur
}

door

\section{Frans Hildert de Vries}

geboren op 20 september 1988

te Sneek 
Dit proefschrift is goedgekeurd door de promotoren:

Prof.dr.ir. A.H. van den Boogaard

Dr.ir. H.J.M Geijselaers 



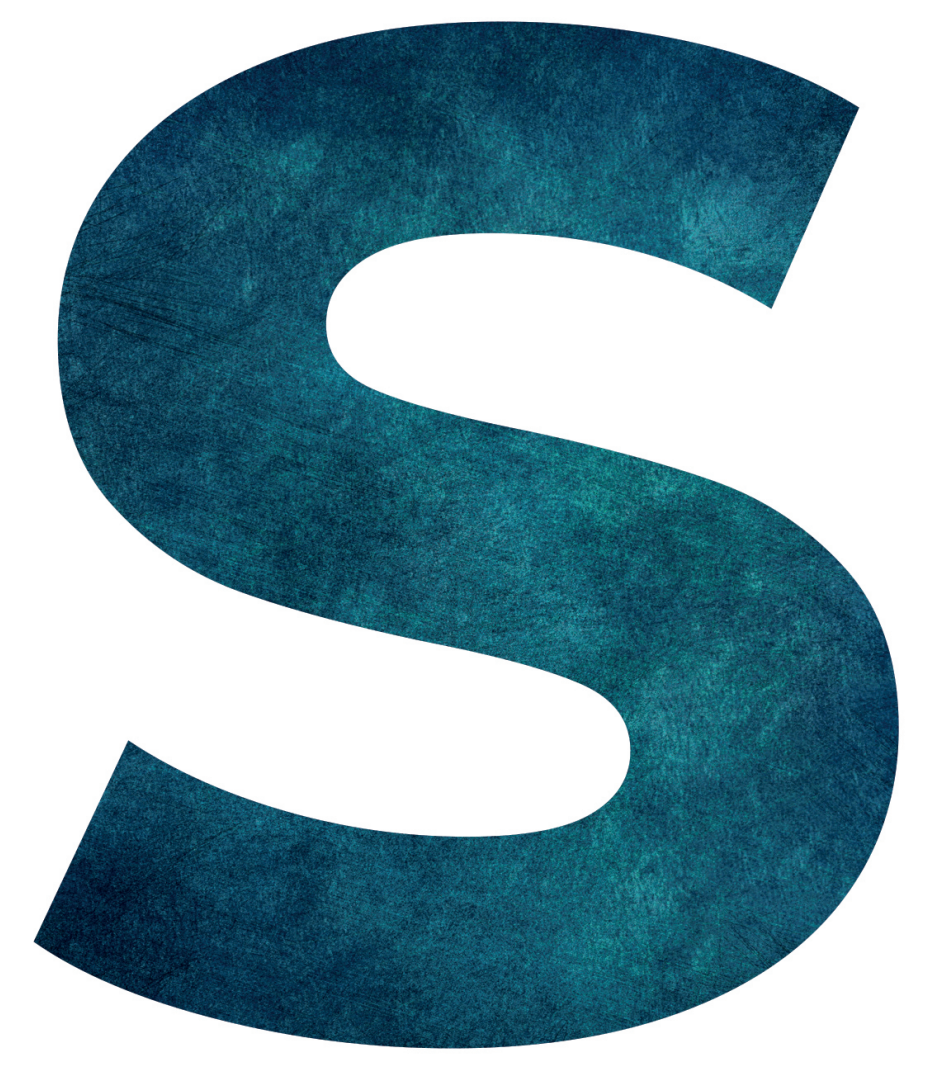




\section{Summary}

All over the world, offshore pipelines are used to transport oil and gas across sea. These pipes are installed on the sea floor by dedicated pipe-laying vessels, for which several laying methods are used. Examples of such methods are the J-lay method and S-lay method, of which the first letter refers to the shape of the pipe during installation. To ensure the integrity of the pipe, pipe-laying is simulated numerically. In this thesis, a numerical model for simulation of offshore pipe-laying is presented. This model has been developed to be substantially more efficient and physically more realistic than existing models.

In the numerical model the pipe is modelled with beam finite elements. These elements can be subjected to large displacements and rotations, which result in geometrically non-linear behaviour. A convenient approach to modelling this nonlinearity is the co-rotational formulation, in which a local coordinate system moves and rotates with the element. In this research, an existing co-rotational beam element formulation has been improved by increasing accuracy and efficiency.

Due to the non-linear relations in the numerical model, an iterative algorithm is required to obtain balance of forces and moments. In this work the NewtonRaphson algorithm is used to obtain quadratic convergence. This requires deriving consistent stiffness matrices to account for variations of all loads that are dependent on the degrees of freedom.

Static loads included are gravity and buoyancy, for which equivalent nodal forces and moments are derived. A detailed buoyancy model is compared with a simpler submerged weight model, which is based on Archimedes' law. The submerged weight model is inaccurate when plastic deformation occurs and when a free pipe end is involved.

Hydrodynamic loads are modelled by Morison's equation, modified for a moving cylinder. This equation consists of three parts: drag, added mass and a pressure gradient. The drag results in damping, the remaining parts are inertia terms.

Dynamic simulations are performed with the HHT- $\alpha$ time integration method. This is an implicit method, for which stability is not dependent on time step size. The HHT- $\alpha$ method is second-order accurate and has controllable numerical 
damping that damps higher frequencies without affecting the lower frequencies too much.

At the start of a dynamic numerical simulation, a load is suddenly applied to the structure. This sudden load can excite high natural frequencies that are physically not relevant. An initialisation procedure for the HHT- $\alpha$ method is presented, that avoids excitation of these high frequencies to prevent incorrect predictions of the pipe's kinematics.

In the pipe-lay model the pipe can be in contact with the seabed and with roller-boxes on the stinger and on the vessel. This contact is modelled with the Penalty method, in which the contact state is binary: there is either 'contact' or 'no contact'. The contact state can alternate between 'contact' and 'no contact' over consecutive Newton-Raphson iterations. A contact-state delay procedure is presented that prevents this alternating behaviour.

A tensioner controls the tension applied to the pipe by paying in or paying out the pipe when the measured tension exceeds predefined limits. In the tensioner model the tension is measured at the tensioner, while the pipe is payed in or out at the end of the stinger. This separation successfully ensures that the pipe nodes that are in contact with the roller-boxes remain in a correct position when the pipe moves axially.

In a thin-walled pipe stresses in radial direction are much smaller than stresses in circumferential and axial direction, such that a plane stress state can be assumed. Stresses in circumferential direction are defined by the internal and external pressure acting on the pipe. These assumptions are used to derive an efficient dedicated algorithm for plasticity in pipes with arbitrary isotropic hardening. Additionally, the plasticity algorithm is implemented such that it does not negatively affect the Newton-Raphson iteration behaviour.

Results of linear elastic J-lay simulations with the new pipe-lay model are similar to those of industry standard software Offpipe. Results of linear elastic S-lay simulations show that Offpipe overestimates bending strains for pipes with a small diameter. When plastic deformation occurs, Offpipe underestimates axial strains due to its simplistic material model. In the new pipe-lay model, these strains are calculated correctly.

The new pipe-lay model can also be used to simulate a start-up procedure with a free pipe end, which requires the detailed buoyancy model. The new pipe-lay model can also be used to model residual curvature in the pipe, which results from the plastic deformation on the stinger. These simulations are not possible in Offpipe.

In the new pipe-lay model numerical time integration can be applied with time steps that are up to 100 times larger than those in Offpipe. These larger time steps are possible due to high stability of the algorithms described in this thesis, such as the contact-state procedure and the implemented plasticity model. 



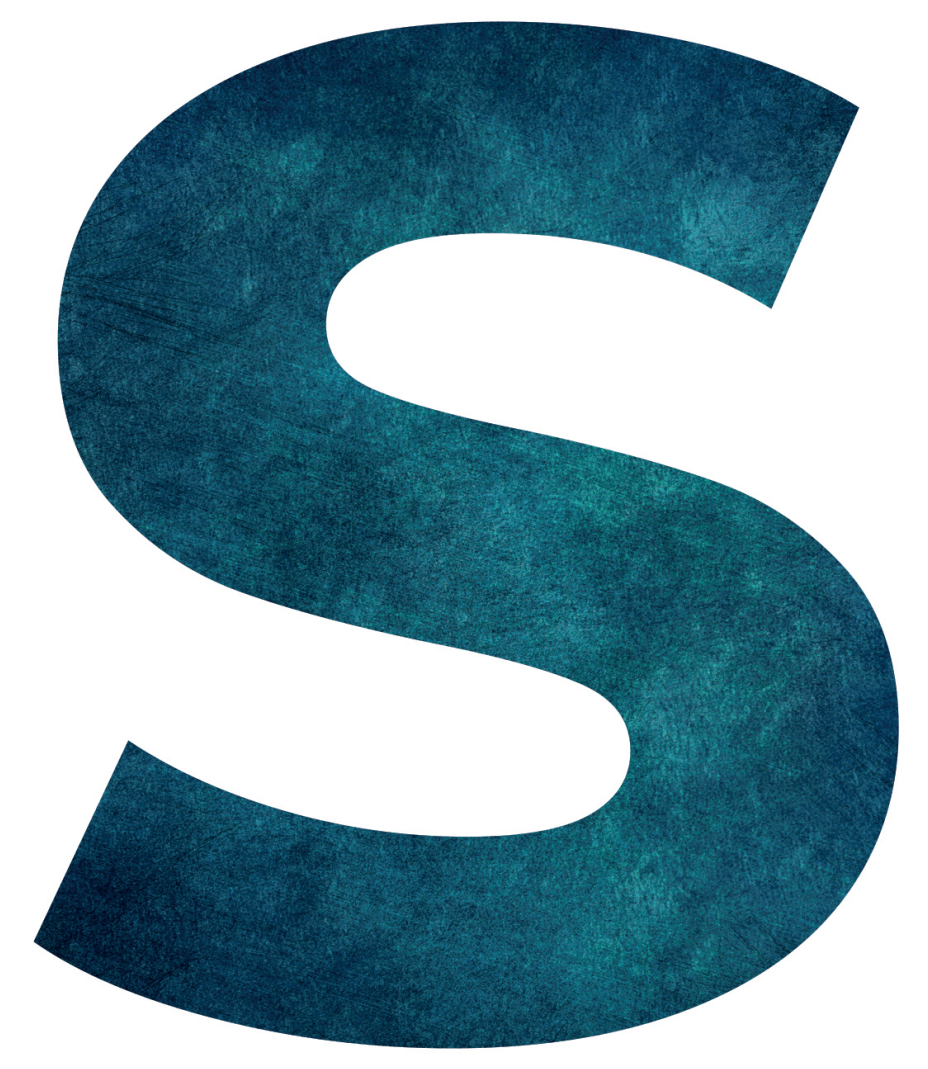




\section{Samenvatting}

Offshore pijpleidingen worden over de gehele wereld gebruikt om olie en gas over zee te transporteren. Deze pijpen worden op de zeebodem geïnstalleerd door speciale pijp-leggende schepen. Deze schepen maken gebruik van verschillende legmethoden, zoals de J-lay-methode en S-lay-methode. De eerste letter van deze methoden beschrijft de vorm van de pijp tijdens installatie. Om de deugdelijkheid van de pijp te waarborgen, wordt het leggen van pijpleidingen numeriek gesimuleerd. In dit proefschrift wordt een numeriek model voor zulke simulaties beschreven. Dit model is ontwikkeld om aanzienlijk efficiënter en fysisch realistischer te zijn vergeleken met bestaande modellen.

De pijp is in het numerieke model gemodelleerd met eindige balkelementen. Deze elementen kunnen grote verplaatsingen en rotaties ondergaan, wat resulteert in geometrisch niet-lineair gedrag. Een geschikte benadering om deze niet-lineariteit te modelleren is de co-rotational formulering, waarbij een lokaal coördinatensysteem verplaatst en roteert met het element. In dit onderzoek is een bestaande co-rotational formulering verbeterd door nauwkeurigheid en efficiëntie te verhogen.

Door de niet-lineaire relaties in het numerieke model is een iteratief algoritme nodig om kracht- en momentbalans te bepalen. In dit proefschrift is gekozen voor het Newton-Raphson-algoritme. Om kwadratische convergentie te verkrijgen, zijn consistente stijfheidsmatrices vereist voor belastingen die afhankelijk zijn van de vrijheidsgraden.

In dit proefschrift zijn de statische belastingen zwaartekracht en drijfvermogen meegenomen, voor deze belastingen zijn equivalente knooppuntskrachten en -momenten afgeleid. Een gedetailleerd model voor waterdruk op de pijp is vergeleken met een eenvoudiger model voor het gewicht van de pijp onder water, dat is gebaseerd op de wet van Archimedes. Het onderwatergewichtsmodel is onnauwkeurig wanneer er plastische vervorming optreedt en wanneer een uiteinde van de pijp vrij kan bewegen.

Hydrodynamische belastingen zijn gemodelleerd met de vergelijking van Morison, aangepast voor een bewegende cilinder. Deze vergelijking bestaat uit drie delen: weerstand, toegevoegde massa en een drukgradiënt. De weerstand zorgt voor demping, de overige delen zijn traagheidstermen. 
De HHT- $\alpha$ methode voor tijdsintegratie is geïmplementeerd voor dynamische simulaties. Dit is een impliciete methode, waarbij de stabiliteit niet afhankelijk is van de tijdstapgrootte. Deze methode is tweede orde nauwkeurig en heeft regelbare numerieke demping, die hogere frequenties dempt zonder de lagere frequenties te veel te beïnvloeden.

Aan het begin van een dynamische numerieke simulatie wordt plotseling een belasting op de constructie gezet. Deze plotselinge belasting kan hoge natuurlijke frequenties opwekken die fysisch niet relevant zijn. $\mathrm{Er}$ is een initialisatieprocedure voor de HHT- $\alpha$ methode gepresenteerd, die het exciteren van deze hoge frequenties onderdrukken. Zo worden onjuiste voorspellingen van de kinematica van de pijp voorkomen.

De pijp kan in contact komen met de zeebodem en met roller-cassettes op de stinger en op het schip. Dit contact is gemodelleerd met de Penalty-methode, waarbij de contacttoestand binair is: er is 'contact' of 'geen contact'. De contacttoestand kan alterneren over opeenvolgende Newton-Raphson iteraties. Er is een contacttoestand-vertragingsprocedure ontwikkeld, die dit alternerende gedrag voorkomt.

Een tensioner regelt de axiale kracht die op de pijp wordt uitgeoefend door de pijp in te halen of te vieren wanneer de gemeten kracht de vooraf gedefinieerde limieten overschrijdt. In het tensionermodel wordt de kracht gemeten bij de tensioner, terwijl de pijp aan het uiteinde van de stinger wordt ingehaald of gevierd. Deze scheiding zorgt er met succes voor dat de knooppunten van de pijp die in contact komen met de roller-cassettes in de juiste positie blijven wanneer de pijp axiaal beweegt.

In een dunwandige pijp zijn spanningen in radiale richting veel kleiner dan spanningen in omtreks- en axiale richting, zodat een vlakspanningstoestand kan worden aangenomen. Spanningen in omtreksrichting worden bepaald door de interne en externe druk op de pijp. Deze aannames zijn gebruikt om een efficiënt algoritme voor plasticiteit af te leiden voor pijpen met willekeurige isotrope versteviging. Bovendien is het plasticiteitsalgoritme zodanig geïmplementeerd dat het iteratiegedrag van het Newton-Raphson algoritme niet negatief wordt beïnvloed.

Resultaten van lineaire elastische J-lay-simulaties met het nieuwe model voor pijpleggen zijn vergelijkbaar met die van de standaardsoftware Offpipe. Resultaten van lineaire elastische S-lay-simulaties tonen aan dat Offpipe buigrekken voor buizen met een kleine diameter overschat. Als gevolg van een simplistische materiaalmodel onderschat Offpipe axiale rekken bij plastische vervormingen. In het nieuwe model voor pijpleggen worden deze rekken correct berekend.

Het nieuwe model voor pijpleggen kan worden gebruikt om een opstartprocedure met een vrij beweegbaar uiteinde van een pijp te simuleren. Hiervoor is het gedetailleerde model voor waterdruk op de pijp nodig. Het nieuwe model voor 
pijpleggen kan ook worden gebruikt om de restkromming in de pijp te modelleren, welke een gevolg is van plastische vervormingen op de stinger. Deze simulaties zijn niet mogelijk met Offpipe.

In het nieuwe model voor pijpleggen kan numerieke tijdintegratie worden toegepast met tijdstappen die tot 100 keer groter zijn dan die in Offpipe. Deze grotere tijdsstappen zijn mogelijk vanwege de hoge stabiliteit van de algoritmes die in dit proefschrift zijn beschreven, zoals de contacttoestandsprocedure en het geïmplementeerde plasticiteitsmodel. 


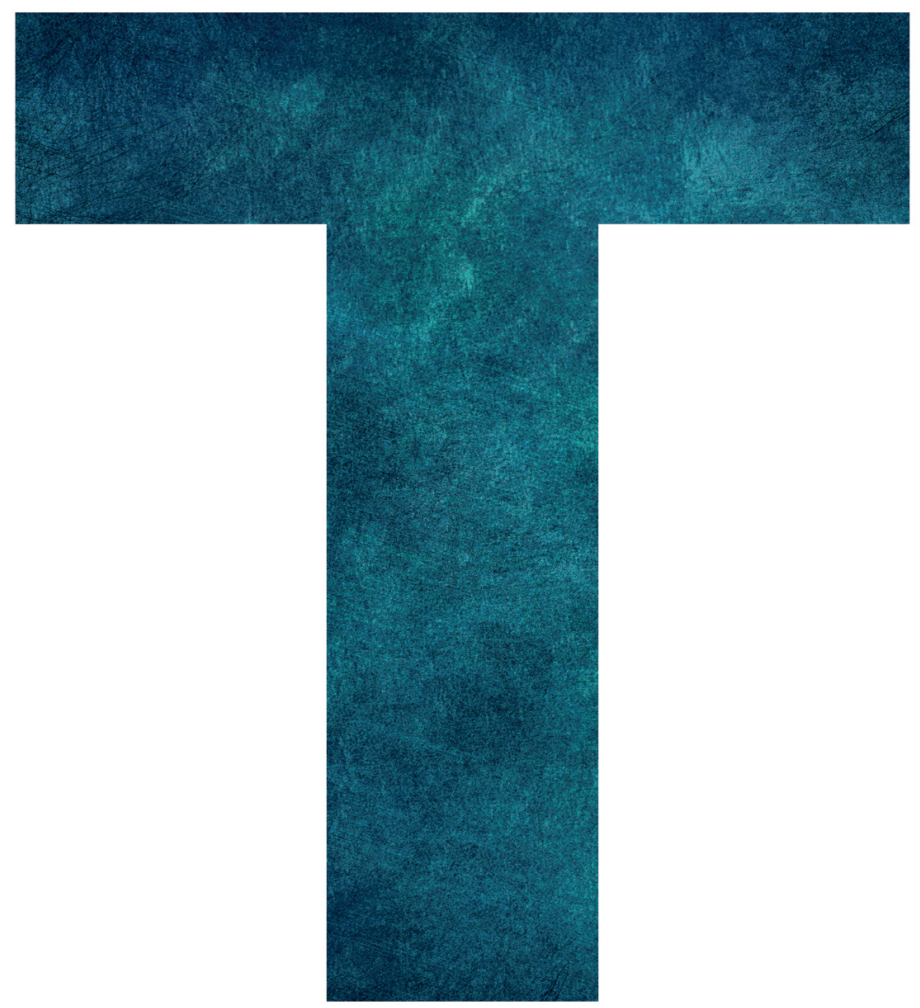




\section{Table of Contents}

Summary $v$

Samenvatting ix

1 Introduction 1

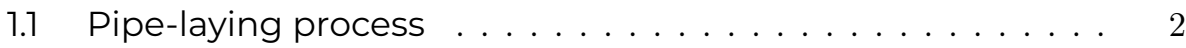

1.1.1 S-lay method ..................... 2

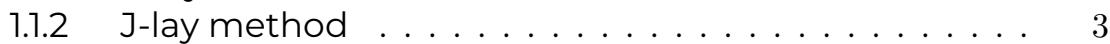

1.2 Numerical modelling of pipe-laying . . . . . . . . . . . . . . . . . 4

1.3 Research objective . . . . . . . . . . . . . . . . . . . . . 5

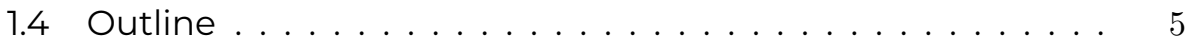

2 Geometrically nonlinear beam element 9

2.1 Element formulation . . . . . . . . . . . . . . . . . 10

2.1.1 Local degrees of freedom . . . . . . . . . . . . . . . . . . . 12

2.1.2 Transformation matrix . . . . . . . . . . . . . . 15

2.1 .3 Rotation matrix . . . . . . . . . . . . . . . . . . . . . . . . . . . . . . 16

2.1.4 Local material stiffness . . . . . . . . . . . . . . . . . . . . . . . . . . . . . . . . . . .

2.1 .5 Global stiffness matrix . . . . . . . . . . . . . . . . 20

2.2 Comparison of element formulations . . . . . . . . . . . . . . 22

2.2.1 Example 1: Two-dimensional circle . . . . . . . . . . . . . 23

2.2.2 Example 2: Helical beam .............. . . 24

2.3 Newton-Raphson algorithm . . . . . . . . . . . . . . . . . . 27

2.4 Distributed forces . . . . . . . . . . . . . . . . . . . . 28

2.5 Second-order axial deformation terms ........... 30

2.5.1 Derivation axial strain . . . . . . . . . . . . . . . . . 30

2.5.2 Element forces and moments . . . . . . . . . . . . 31

2.5 .3 Necessity of second-order terms . . . . . . . . . . . 34

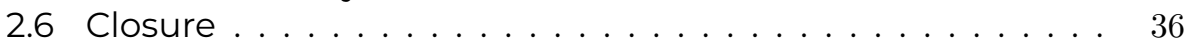

3 Numerical time integration 39

3.1 Method selection . . . . . . . . . . . . . . . . . . . 40 
3.1.1 Newmark- $\beta$ method . . . . . . . . . . . . . . . . . 42

3.1.2 Hilber-Hughes-Taylor- $\alpha$ method . . . . . . . . . . . . . 44

3.1.3 Generalised- $\alpha$ method . . . . . . . . . . . . . . . . 45

3.1 .4 Comparison . . . . . . . . . . . . . . . . . . . 47

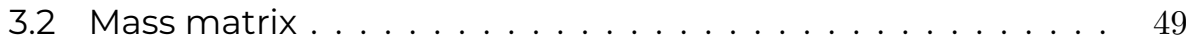

3.2 .1 Consistent . . . . . . . . . . . . . . . . . . . . . . . . . . . . . . . . 49

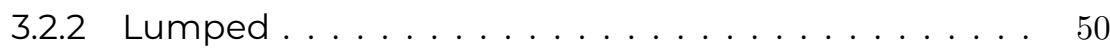

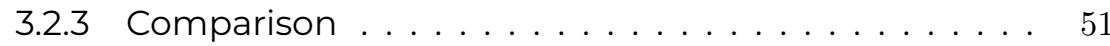

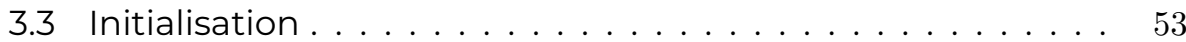

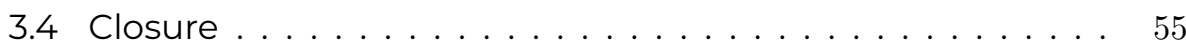

4 Contact modelling 59

4.1 Penalty method . . . . . . . . . . . . . . . . . . . . 60

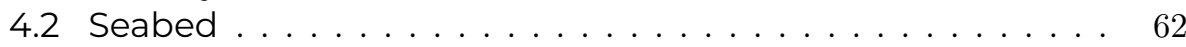

4.3 Roller-boxes. . . . . . . . . . . . . . . . . . . . . . 62

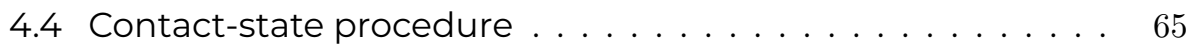

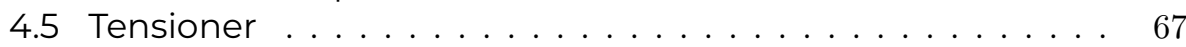

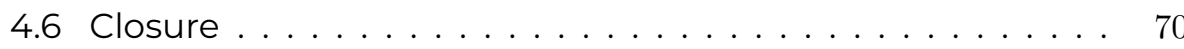

5 Loads and prescribed displacements $\quad 73$

5.1 Hydrostatic loads . . . . . . . . . . . . . . . . . . . . . . . . . . . . . . 74

5.1 .1 Submerged weight . . . . . . . . . . . . . . . . . . . . . . . . . . . 75

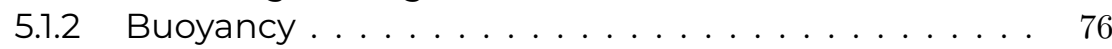

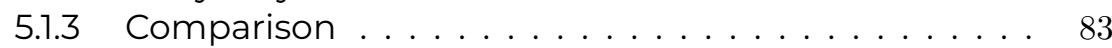

5.2 Hydrodynamic loads . . . . . . . . . . . . . . . . . . . . . . . . . . . . . 85

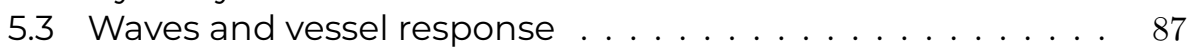

5.3 .1 Regular wave . . . . . . . . . . . . . . . . . . . . . . . . 89

5.3 .2 Generic wave . . . . . . . . . . . . . . . . . . . . . . . . . . . 90

5.3 .3 Vessel response. . . . . . . . . . . . . . . . . . . . . . . . . . . . 91

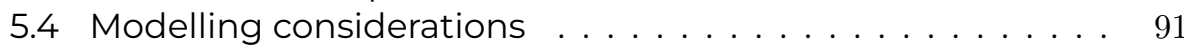

5.4 .1 Maximum time step size . . . . . . . . . . . . . . . . 92

5.4.2 Axial compression in submerged elements . . . . . . . . 94

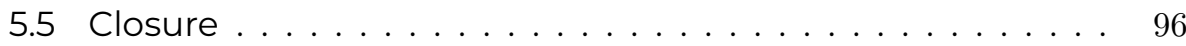

6 Plasticity 99

6.1 Dedicated algorithm for plasticity in pipes . . . . . . . . . . . . . . 99

6.1.1 Return mapping algorithm . . . . . . . . . . . . . 100

6.1 .2 Validation . . . . . . . . . . . . . . . . . 107

6.1 .3 Discussion . . . . . . . . . . . . . . . . . . 109

6.2 Implementation in co-rotational elements . . . . . . . . . . . . 110

6.2.1 Integration over the volume of an element . . . . . . . 110

6.2.2 Algorithm for co-rotational elements . . . . . . . . . . . 114

6.2.3 Second-order terms due to bending and plasticity . . 115

6.3 Closure ............................. 118 
7 Pipe-lay examples 121

7.1 J-lay procedure . . . . . . . . . . . . . . . . . . 122

7.1 .1 Static simulation . . . . . . . . . . . . . . . . . . . 123

7.1 .2 Dynamic simulation . . . . . . . . . . . . . 125

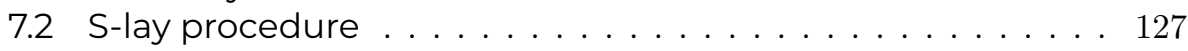

7.2 .1 Static simulation . . . . . . . . . . . . . . . . . . . . 129

7.2 .2 Dynamic simulation . . . . . . . . . . . . . . 131

7.3 Start-up with a free pipe end . . . . . . . . . . . . . 134

7.4 Closure . . . . . . . . . . . . . . . . . . 136

8 Conclusions and recommendations 139

8.1 Conclusions . . . . . . . . . . . . . . . . . . . . . . . 139

8.2 Recommendations . . . . . . . . . . . . . . . . . 142

A Contributions from external loads to the stiffness matrix $\quad 147$

A.1 Buoyancy . . . . . . . . . . . . . . . . . . . . . . . . . 147

A.1.1 Distributed pressure . . . . . . . . . . . . . . . . . 148

A.1.2 Curvature effects . . . . . . . . . . . . . . . . . . . . . 149

A.1.3 Capped ends . . . . . . . . . . . . . . . . . . 150

A.1.4 Correction on cross-sectional area . . . . . . . . . . . 151

A.2 Hydrodynamic loads . . . . . . . . . . . . . . . . . . 151

A.2.1 Water velocities . . . . . . . . . . . . . . . . . . . 151

A.2.2 Water accelerations . . . . . . . . . . . . . . . 152

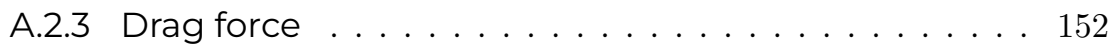

A.2.4 Added mass . . . . . . . . . . . . . . . . . . . . 153

A.2.5 Pressure gradient . . . . . . . . . . . . . 154

$\begin{array}{ll}\text { References } & 157\end{array}$ 


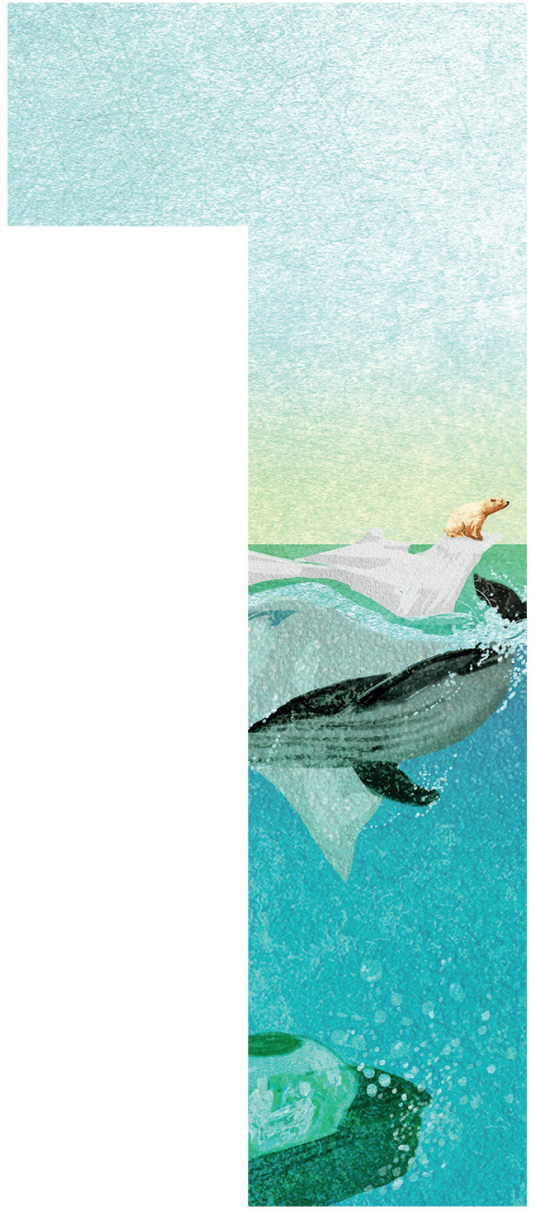




\section{1 \\ Introduction}

The world energy consumption has been growing for centuries. As shown in Figure 1.1, it is expected that people consume approximately 14 billion tonne of oil equivalent (toe) in 2020 , which is equivalent to 0.59 zettajoule $\left(10^{21} \mathrm{~J}\right)$ or 163 trillion kWh. More than half of this energy is harnessed from oil or gas. These energy carriers need to be transported, for example from production site to refineries. Oil and gas are normally transported by pipelines, trains, trucks or vessels. Of these transportation options, pipelines are the most economical and reliable means of transport.

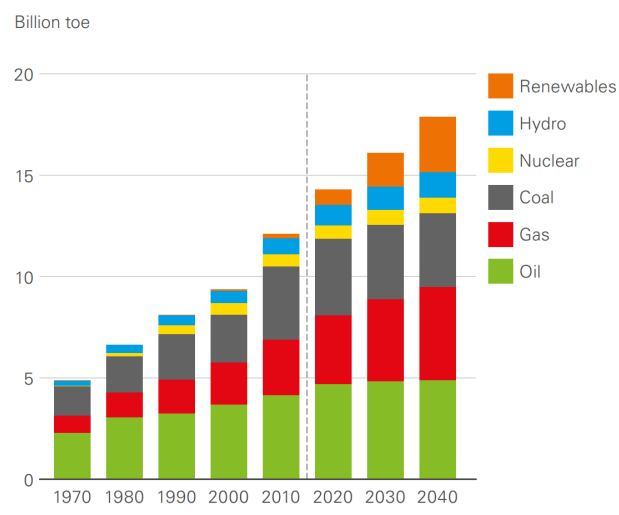

Figure 1.1: World energy consumption by fuel type [8].

The first offshore pipelines were installed in 1944, during the Second World War. In operation PLUTO (PipeLines Under The Ocean), the Allies set their sights on an innovative, reliable fuel supply from southern England to their armies in France, as conventional oil tankers were easy targets for German submarines and the Luftwaffe [56]. After some trial and error, they installed a number of 
prefabricated pipes by unrolling them from a large floating drum, which rolled across the English Channel.

More modern pipe-laying methods are presented in Section 1.1. Nowadays, instead of trial and error, numerical models of pipe-laying operations ensure the integrity of the pipeline. This thesis focuses on improving accuracy and efficiency of these numerical models.

\subsection{Pipe-laying process}

Several pipe-laying methods are used by the offshore industry, such as tow-in, reellay, J-lay and S-lay. Detailed descriptions of these methods can be found in [25]. The current work focuses on the S-lay and J-lay methods.

\subsubsection{S-lay method}

The S-lay method is named after the pipe's characteristic S-shape while being laid, as can be seen in Figure 1.2. The remainder of this section will give a further introduction to the S-lay method.

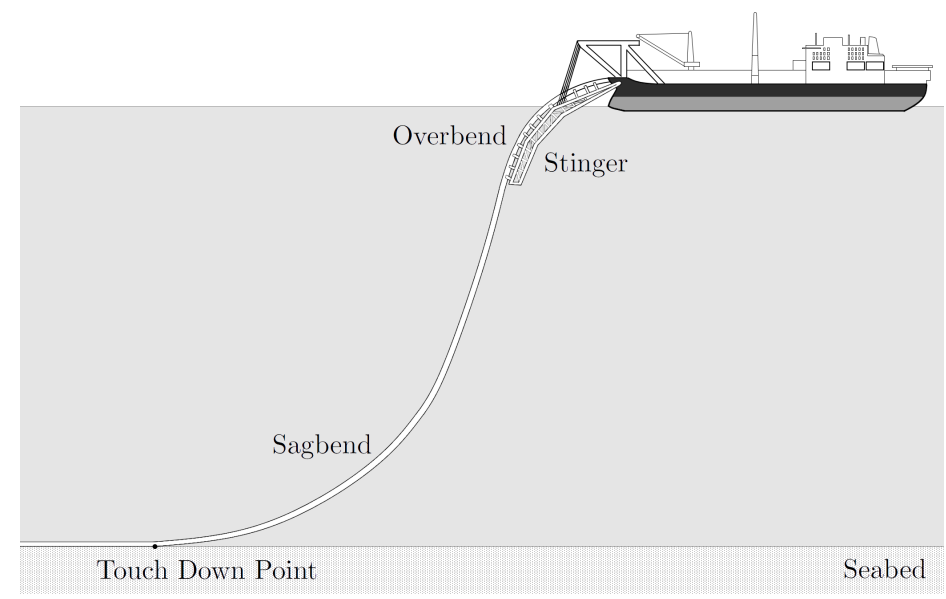

Figure 1.2: Schematic overview of the S-lay method.

On board the S-lay vessel, the pipe is assembled from shorter segments. These segments are delivered to the pipe-laying vessel by a supply barge and are transferred to the onboard storage by the vessel's cranes. From storage, the pipes are transferred to a firing line, where the pipe segments are welded together, inspected and coated. 
At the end of the firing line the pipe is held in place by one or more tensioners, which apply an axial tension to the pipe. These tensioners can pay out the pipe after a new pipe segment has been welded on. The tensioners can also pay out or pay in the pipe when the axial tension exceed certain predefined upper or lower bounds.

The pipe leaves the vessel over a stinger, which is a truss structure hanging from the stern or bow of the vessel. On the stinger, several roller-boxes support the pipe. The pipe section above the stinger is commonly referred to as the overbend. Here, pipe deformations are determined mainly by the shape of the stinger.

Moving further towards the sea floor, the pipe passes through a section called the sagbend, which ends at the touch down point on the seabed. The shape of the sagbend is determined mainly by the axial tension applied by the tensioners. Lowering the tension will result in a smaller bending radius, increasing the tension will result in a larger bending radius.

To ensure that pipe deformations in the sagbend stay within predefined limits, the contact force of the last roller-box can be monitored. Alternatively, if there is no contact between the last roller-box and the pipe, the distance between the pipe and the last roller-box can be monitored. This distance is called the tip separation.

An advantage of the S-lay method is that it can be applied in both shallow and deep water, with small and large diameter pipes. In the horizontal firing line, welding, inspection and coating stations can be placed in series, resulting in a relative high laying speed. A disadvantage of the method is that laying large diameter pipes in deep water requires a long stinger with a large radius, to prevent excessive deformation of the pipe in the overbend. Overall, S-lay is an efficient and widely applied method for pipe-laying.

\subsubsection{J-lay method}

The J-lay method is named after the pipe's characteristic J-shape while being laid. As for the S-lay method, the pipe is assembled from shorter segments on board the pipe-laying vessel. There are dedicated vessels designed for J-lay operations [25], which have a J-lay tower on board instead of a stinger. In this vertical tower, pipe sections are welded together, after which the pipe is lowered to the seabed in the J-shape.

In special cases, S-lay vessels use the J-lay technique without the vertical tower, for instance when an in-line structure must be installed that is too large to fit through the tensioners. An example of such an in-line structure is a manifold that connects a main pipeline to one or more branch lines.

To install this in-line structure, the pipe end on the stinger is lowered to the seabed. Subsequently, it is recovered in J-lay mode, in which the pipe end is hoisted up by a cable towards a J-lay hinge on the side of the vessel. Here, the in-line structure is connected to the pipe, after which it is lowered back on the seabed. 
In this thesis only J-lay procedures with a J-lay hinge are considered; those with a J-lay tower are out of scope.

\subsection{Numerical modelling of pipe-laying}

Numerical simulations of offshore pipelaying are typically performed using a Finite Element approach, in which the pipe is modelled using beam elements. These models are non-linear due to large rotations, due to contact with stinger and seabed, and due to non-linear material behaviour. Therefore, the static simulation is carried out with an iterative method. Dynamic simulations are performed using numerical time integration. Motions of the vessel can be determined from a linearised relation between these motions and the waves of the water surface, the Response Amplitude Operators (RAO's). Commercial software packages are available for the simulation of pipe-laying, such as Offpipe $[35,36]$, Orcaflex [46] and Pipelay [65]. These packages all have their advantages as well as limitations.

A pipe-laying project consists of several operations, such as the start-up at the beginning and laydown at the end of a project's execution. In bad weather conditions, an abandonment and recovery procedure is followed. Bad weather conditions can happen at any point along the pipe's route, which means that an abandonment and recovery procedure must be prepared for all possible water depths. Pipe-laying projects can also require the installation of in-line structures. In the preparation phase of a pipe-laying project safety and integrity of the pipeline must be ensured for all possible wave directions. This means that for each operation simulations are required for an entire wind rose.

The offshore industry follows certain standards, such as [20] for submarine pipeline systems. From this standard it follows that pipe-lay projects require a simulation that covers a period of three hours, which results in high computational costs. This, in combination with the large number of required simulations, justifies the need to reduce computational time of pipe-lay simulations.

Computational costs of a dynamic model can be reduced by choosing larger time steps. Time steps are limited in size due to accuracy, depending on the period of the waves and the response of the vessel and pipe. Subject to the choice of time integration method, time steps can be limited by numerical stability. When the time step is larger than a critical time step, numerical approximation errors are magnified over time. Time steps can also be limited by convergence issues. When the time step increases, the displacement increment also increases. In nonlinear aspects of the model, such as contact and plastic material behaviour, a large displacement increment can cause problems with convergence.

In the previously mentioned software packages, large time steps are possible for simple simulations, such as the simulation of a J-lay process with linear-elastic material. However, when non-linear material behaviour, tensioner control or 
stinger contact are introduced, the time step must be decreased to prevent non-convergence or instability. This increases the simulation time significantly.

Submerged pipes are subject to buoyancy forces, which result from a difference in pressure that acts on the pipe's surface. Existing pipe-lay models use Archimedes' law to determine an upward force. This force is subtracted from the gravity force, resulting in a so called submerged weight. This approach ignores the influence of the pipe's curvature and the pressure acting on the pipe's submerged end. Therefore, when applying Archimedes' law on a submerged pipe, a post-processing step is required where a correction is made to the axial force, known as effective tension [57]. Evaluating the model with an incorrect axial force can lead to inaccurate results.

Existing pipe-lay models include some form of non-linear material behaviour. O'Grady [45] pointed out that most commercially available installation software create moment-curvature and force-displacement curves from a user input material stress-strain curve, prior to the finite element simulation. Commonly, one moment-curvature curve is used for the entire pipe. This incorrectly assumes a constant axial tension and disregards the effects of torsion and pressure, leading to inaccurate results.

\subsection{Research objective}

The primary aim of this work is to develop an accurate numerical model for the simulation of pipe-laying that is substantially more efficient than existing models. An efficient beam element and efficient time integration is investigated. In the implementation of models for contact and plastic material behaviour, a study is made of whether large time steps can be used.

To model hydrostatic forces accurately, a detailed buoyancy model is investigated. This model includes the influence of the pipe's curvature and the pressure acting on the pipe's submerged end. Results obtained using this detailed buoyancy model are compared to those obtained using Archimedes' law with effective tension.

To overcome the issue where models use a single moment-curvature relation for the entire pipe, a dedicated plasticity model for pipes is developed. This model includes elasto-plastic loading-unloading behaviour. In this model, the pipe can be subject to bending, pressure, normal force and tension. The plasticity model must be computationally inexpensive.

\subsection{Outline}

In the numerical model the pipe will be modelled with beam elements of EulerBernoulli type. In Chapter 2 an efficient beam element is investigated. An existing 
beam element from literature is modified to increase efficiency and accuracy. The modified beam element is also presented in a recently submitted journal paper ${ }^{1}$.

In Chapter 3 requirements for the numerical time integration method are defined. The method that best fits these requirements is selected from literature. In the start-up of pipe-lay simulations an overshoot issue occurs. An initialisation method is presented to overcome this issue.

In Chapter 4 the model for contact between the pipe and the stinger and between the pipe and the seabed is presented. A dedicated procedure ensures that the contact model does not decrease efficiency. The model for contact between the pipe and the seabed is also part of journal paper [18], co-authored by H.J.M Geijselaers, A.H. van den Boogaard and A. Huisman.

In Chapter 5 external loads and prescribed displacements of the pipe-laying vessel are presented. Displacements of the pipe are dependent on these external loads, which include gravity, hydrostatic and hydrodynamic loads. Displacements of the pipe-laying vessel are prescribed by a response to waves at the water surface. The model for these external loads are also part of a conference paper [17] and a subsequent journal paper [18], both co-authored by H.J.M Geijselaers, A.H. van den Boogaard and A. Huisman.

In Chapter 6 a numerical plasticity model for pipes is presented. Results of this new model are compared to those of a radial return mapping algorithm. The plasticity model is also presented in a recently submitted journal paper ${ }^{2}$.

In Chapter 7 results of pipe-lay simulations with the new numerical model are compared to results of industry standard software Offpipe. This chapter includes J-lay and S-lay simulations.

Finally, in Chapter 8 the conclusions drawn from this study are summarised and recommendations for future research are presented.

\footnotetext{
${ }^{1}$ F.H. de Vries, H.J.M. Geijselaers and A.H. van den Boogaard. An efficient 3D corotational beam element for slender structures, Submitted, 2020

${ }^{2}$ F.H. de Vries, H.J.M. Geijselaers and A.H. van den Boogaard. Return mapping algorithm for elasto-plastic deformation of thin-walled pipes, Submitted, 2020
} 



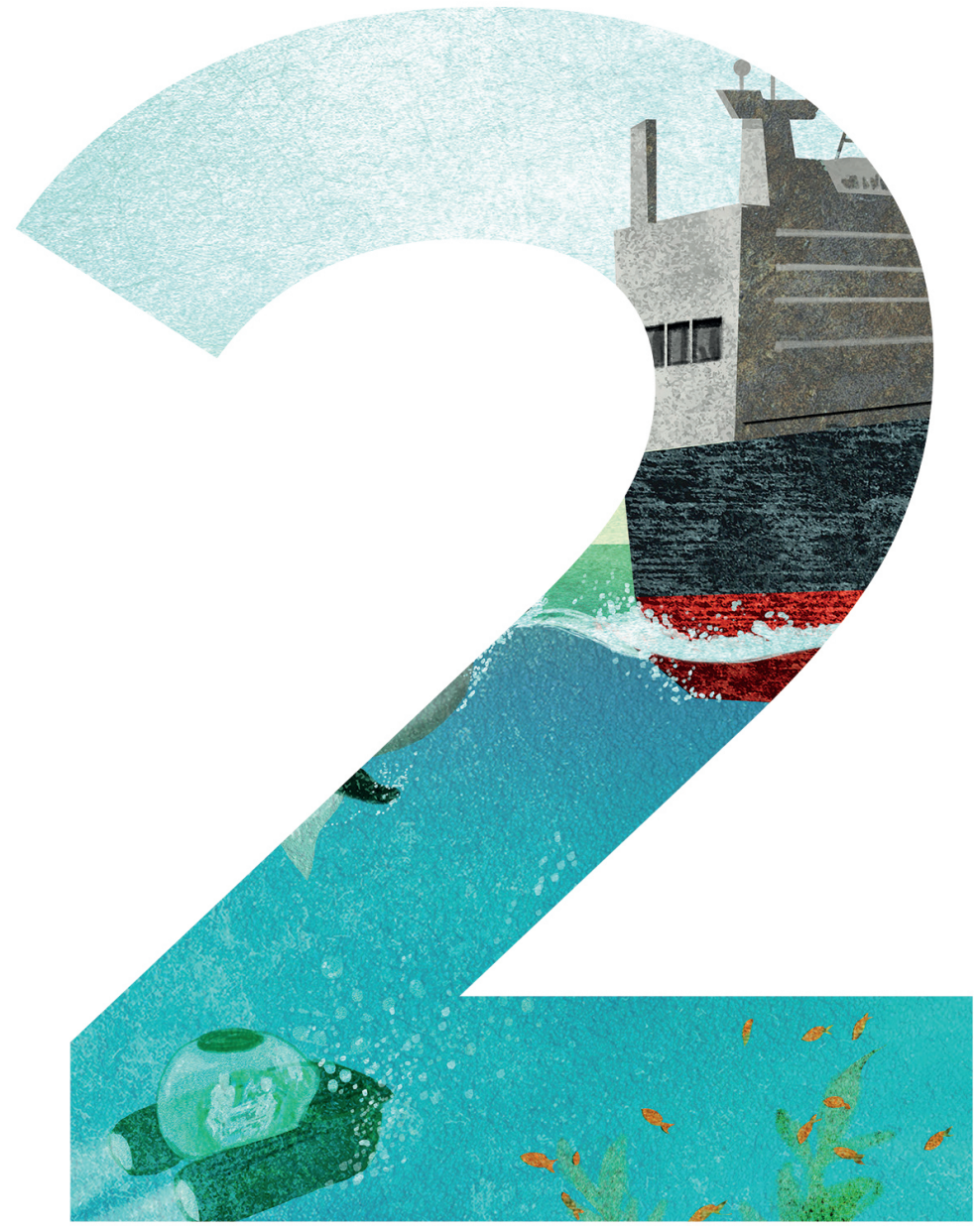




\section{Geometrically nonlinear beam element}

In offshore pipe-laying large displacements and rotations of the pipe often occur. In numerical models of pipe-laying internal forces and moments are nonlinear functions of the displacements and rotations. A popular approach to modelling these nonlinearities is the co-rotational method, which uses a beam finite element with a local coordinate system that moves and rotates with the element. The primary advantage of this method, as agreed on by most authors $[6,13,23]$, is the possibility to use efficient linear deformation theory within the local coordinate system. In this chapter a new and efficient co-rotational beam element is presented, based on De Vries et al. ${ }^{1}$

The idea of a coordinate system that moves and rotates with respect to the global coordinates originates from before the FEM era [67, 68], and was originally named 'le système de coordonnées mobile'. The first use of a co-rotational approach in a finite element context is attributed to Belytschko [7]. Crisfield $[14,15]$ developed a three dimensional co-rotational beam element. He focused on a consistent tangent stiffness matrix, which he found to be generally non-symmetric due to the non-commutativity of three dimensional rotations. Near convergence the tangent stiffness matrix becomes symmetric, which makes artificially symmetrising the matrix a possibility for enhancing efficiency [13].

Simultaneously, Rankin and Brogan [50] and later Nour-Omid and Rankin [44] developed a co-rotational formulation that is element independent. This means that it is not restricted to beam elements but is also applicable to plate elements. Pacoste and Erikson [47] extended this formulation with an additive rotational vector, based on the work of Ibrahimbegović et al. [33], to describe the three

\footnotetext{
${ }^{1}$ F.H. de Vries, H.J.M. Geijselaers and A.H. van den Boogaard. An efficient 3D corotational beam element for slender structures, Submitted, 2020
} 
dimensional rotations. Battini and Pacoste [6] continued development of this formulation, which in the remainder of this chapter is referred to as the element formulation of Battini-Pacoste. They introduced a two-step approach to determining the global force vector and stiffness matrix, which allows for any local constitutive model, including nonlinear material models with large strains. Battini and Pacoste also pointed out that co-rotational beam element formulations that assume linear deformation theory within the local model have restrictions for certain problem types. In particular they pointed out that these formulations are unable to predict correct results when torsion is involved. When the formulation of Battini-Pacoste is applied to problems that involve torsion, correct results are obtained.

In Section 2.1 a formulation for efficient Euler-Bernoulli type beam elements in a three dimensional co-rotational setting is presented. These elements can handle large displacements and rotations. The basis for this new approach is the beam element formulation of Crisfield, which is made more efficient by changing its most computationally intensive part. This part is the geometric stiffness matrix, which is derived from the rotation matrix. By introducing an alternative rotation matrix, a computationally inexpensive geometric stiffness matrix is derived. The alternative rotation matrix is defined such that components of moments are continuous at each node. This prevents incorrect results when large torsional moments are present, as will be explained in Section 2.1.3. Accuracy and efficiency of the new element formulation are compared to that of the original formulation of Crisfield and to that of Battini-Pacoste.

Because the relation between an element's degrees of freedom and its internal forces is nonlinear, an iterative method is required to obtain force equilibrium. In this thesis the Newton-Raphson algorithm is used as iterative method for static simulations, which is presented in Section 2.3. In Chapter 3 the co-rotational formulation is combined with a time integration method for dynamic simulations.

Loads can be applied only on the nodes of an element. In the pipe-lay simulation a uniformly distributed load due to gravity acts on the pipe. In Section 2.4 it is described how uniformly distributed loads can be applied using the co-rotational formulation.

In Section 2.1 it is assumed that the deformed length of an element is defined by the distance between its nodes. When an element is curved, this is an approximation. The correct length can be taken into account by second-order terms in the axial strain, which are derived in Section 2.5. With these terms the local constitutive model becomes nonlinear.

\subsection{Element formulation}

When rotations of beam elements are large, the relation between an element's degrees of freedom and its internal forces becomes nonlinear. This geometric 
nonlinearity can be accounted for by separating deformations from rigid translations and rotations by using a local coordinate system that translates and rotates with the element. In this local coordinate system, local forces and moments are determined from local degrees of freedom. These local forces are rotated to a global coordinate system. It is assumed that deformations in the local coordinate system are small. In this chapter a linear elastic deformation model is used to determine the local forces and moments from the local degrees of freedom. This model can be substituted by any linear or nonlinear local deformation model in which small deformations are assumed.

The three dimensional co-rotational beam element has three translational and three rotational degrees of freedom at each node, hence it has a total of twelve degrees of freedom. Due to the separation between deformations and rigid translations and rotations, the number of local degrees of freedom is smaller. The original element of Crisfield [15] has seven local degrees of freedom, which are presented in Section 2.1.1.

The original element formulation of Crisfield uses a transformation matrix $\mathbf{B}$ to transform forces from the local to the global coordinate system. This transformation matrix is obtained from the derivative of the local degrees of freedom to the global degrees of freedom. It is not a proper rotation matrix because it is not orthogonal: $\mathbf{B}^{\mathrm{T}} \mathbf{B} \neq \mathbf{I}$, where $\mathbf{I}$ is the identity matrix. This transformation matrix is presented in Section 2.1.2.

The new element formulation uses a separate rotation matrix $\mathbf{R}$ to rotate the local forces to the global coordinate system. This rotation matrix is orthogonal: $\mathbf{R}^{\mathrm{T}} \mathbf{R}=\mathbf{I}$. Locally, nodal moments are defined in directions relative to the crosssection. When these moments are not properly rotated, they can partly transfer to a different direction relative to the cross-section. For example, part of the torsional moment can transfer to a bending moment. This will be explained in Section 2.1.3. In the new rotation matrix the rotation is defined such that this transfer does not occur. This is referred to as continuity of moments at the nodes. The importance of continuity of moments at the nodes is demonstrated in Section 2.2.2.

The Newton-Raphson algorithm requires a consistently derived stiffness matrix, which consists of two contributions. The first contribution is referred to as the material stiffness matrix, which describes changes in internal forces by a change in the local degrees of freedom. This material stiffness matrix must be rotated to the global coordinate system. The second contribution is referred to as the geometric stiffness matrix, which is due to changes of the local coordinate system when an element rotates.

The local material stiffness matrix is non-square, because there are seven local degrees, while the local force vector has length 12 . This length is required for the square rotation matrix of size $12 \times 12$. The derivation of the local stiffness matrix is presented in Section 2.1.4.

The global material stiffness matrix is obtained from that in the local coordinate system by a rotation involving both the transformation matrix $\mathbf{B}$ and rotation 
matrix $\mathbf{R}$. The geometrical stiffness matrix is determined from the derivative of rotation matrix $\mathbf{R}$ to the global degrees of freedom. These stiffness matrices are presented in Section 2.1.5.

\subsubsection{Local degrees of freedom}

In three dimensions a single beam element has two nodes, each with six degrees of freedom: three translational and three rotational. In the deformed state of the element the origin of the local coordinate system is placed on the first node and the local $x_{\mathrm{L}}$-axis coincides with both nodes. By definition the displacement in $x_{\mathrm{L}}$-direction is zero at the first node and the displacements in $y_{\mathrm{L}^{-}}$and $z_{\mathrm{L}}$-direction are zero at both nodes. Following Crisfield the seven remaining degrees of freedom of an element are:

$$
\boldsymbol{p}_{\mathrm{L}}=\left[\begin{array}{lllllll}
\psi_{1} & \theta_{1} & \phi_{1} & u_{\mathrm{L}} & \psi_{2} & \theta_{2} & \phi_{2}
\end{array}\right]^{\mathrm{T}}
$$

$\psi_{j}, \theta_{j}$ and $\phi_{j}$ are the rotations about the $x_{\mathrm{L}^{-}}, y_{\mathrm{L}^{-}}$and $z_{\mathrm{L}^{-}}$-axis respectively, as can be seen in Figure 2.1. The subscript refers to the first or second node of the element, and $u_{\mathrm{L}}$ is the local displacement of the second node in local $x_{\mathrm{L}}$-direction.

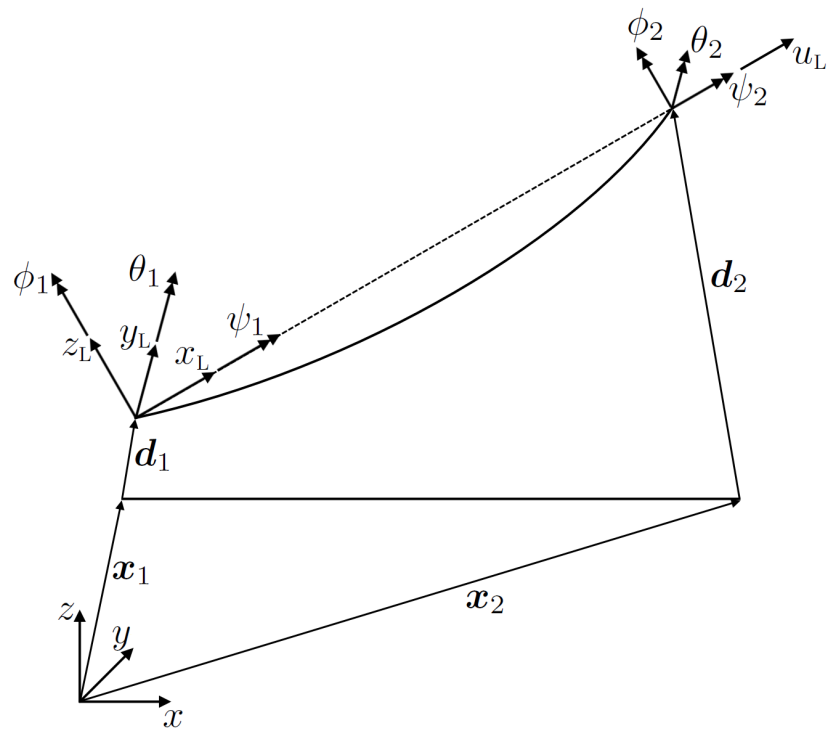

Figure 2.1: Local coordinate system with local degrees of freedom.

The local displacement and rotations are determined from the global displacements and rotations, as shown in the remainder of this section. For convenience and 
following Crisfield, two auxiliary vectors are defined:

$$
\boldsymbol{x}_{21}=\boldsymbol{x}_{2}-\boldsymbol{x}_{1} \quad \boldsymbol{d}_{21}=\boldsymbol{d}_{2}-\boldsymbol{d}_{1}
$$

Here, $\boldsymbol{x}_{j}$ is a vector representing the initial position of node $j$ in the global coordinate system, and $\boldsymbol{d}_{j}$ is a vector representing the displacement of node $j$ in the global coordinate system, as defined in Figure 2.1. The vectors $\boldsymbol{x}_{21}$ and $\boldsymbol{d}_{21}$ can be used to determine the undeformed and deformed lengths of an element:

$$
L_{0}=\left\|\boldsymbol{x}_{21}\right\| \quad L=\left\|\boldsymbol{x}_{21}+\boldsymbol{d}_{21}\right\|
$$

Here, the deformed length of an element is determined by the distance between the nodes. For curved elements, this is an approximation. The correct length of a curved element is presented in Section 2.5. Accounting for the correct length results in higher-order terms in the local deformation model. These terms can be included in all co-rotational element formulations, for example based on Green's strain [14].

A nodal frame $\mathbf{T}$ is assigned to each node, an element frame $\mathbf{E}$ is assigned to each element. The components of element frame $\mathbf{E}$ define the directions of the local coordinate system in Figure 2.1. Each frame is defined by three orthogonal unit vectors:

$$
\mathbf{T}=\left[\begin{array}{lll}
\boldsymbol{t}_{1} & \boldsymbol{t}_{2} & \boldsymbol{t}_{3}
\end{array}\right] \quad \mathbf{E}=\left[\begin{array}{lll}
e_{1} & e_{2} & e_{3}
\end{array}\right]
$$

To distinguish between the nodal frames in the context of an element, the nodal frame at the first node is referred to as $\mathbf{T}$ and that at the second node is referred to as $\mathbf{U}$. In the context of single nodes, the nodal frame is referred to as T. Figure 2.2 shows the three frames $\mathbf{T}, \mathbf{E}$ and $\mathbf{U}$ in the context of a single element and in the context of single nodes.

In each iteration nodal frame $\mathbf{T}$ is updated using an 'incremental spin vector' $\Delta \boldsymbol{\alpha}$. This vector represent the axis of rotation and its norm equals the angle of rotation. This vector is not additive but can be transformed into a rotation matrix $\Delta \mathbf{T}$ by Rodrigues' formula [51]. With this rotation matrix a nodal frame of iteration $n+1$ is determined from that in iteration $n$ :

$$
\mathbf{T}_{n+1}=\Delta \mathbf{T}(\Delta \boldsymbol{\alpha}) \mathbf{T}_{n}
$$

The global degrees of freedom vector of an element is defined by the global displacement vectors and by the incremental spin vectors at both nodes:

$$
\boldsymbol{p}=\left[\begin{array}{llll}
\boldsymbol{d}_{1}^{\mathrm{T}} & \Delta \boldsymbol{\alpha}_{1}^{\mathrm{T}} & \boldsymbol{d}_{2}^{\mathrm{T}} & \Delta \boldsymbol{\alpha}_{2}^{\mathrm{T}}
\end{array}\right]^{\mathrm{T}}
$$

The global degrees of freedom vector contains translational and rotational degrees of freedom. The translational degrees of freedom are additive and indicate the positions of the nodes, as in figure 2.1. The rotational degrees of freedom are not additive but represent the update of the last computed increment, as shown in Equation (2.5). 


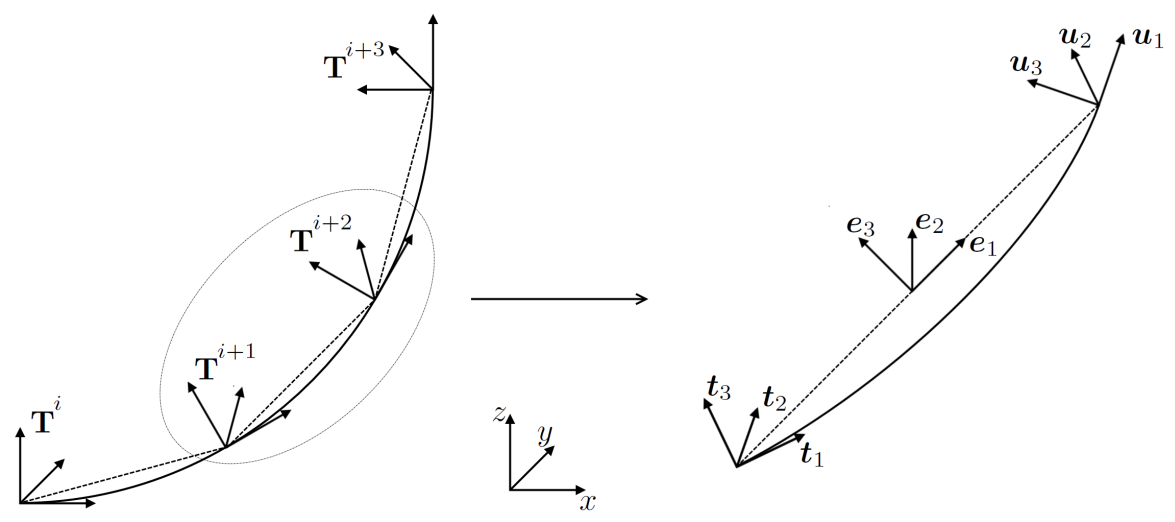

Figure 2.2: Co-rotational beam element with nodal frames of orthogonal unit vectors to define rotations in three dimensions.

In addition to the nodal frames, element frame $\mathbf{E}$ is updated in each iteration [15]. The first vector of this element frame points from the first node to the second, as can be seen in Figure 2.2. It is obtained by:

$$
e_{1}=\frac{1}{L}\left(\boldsymbol{x}_{21}+\boldsymbol{d}_{21}\right)
$$

Next, this element frame is rotated about the $\boldsymbol{e}_{1}$ axis, such that it is halfway between the torsional rotation of the first and the second node. The difference in rotation between the nodes is quantified with a rotation matrix that can rotate the frame of the first node to that of the second node:

$$
\mathbf{R}_{\boldsymbol{\gamma}}=\mathbf{U T}^{\mathrm{T}}
$$

A mean rotation matrix is obtained by rotating the frame of the first node $\mathbf{T}$ by half of the rotation $\mathbf{R}_{\boldsymbol{\gamma}}$, which is determined by extracting spin vector $\boldsymbol{\gamma}$ from $\mathbf{R}_{\boldsymbol{\gamma}}$ using Spurrier's algorithm [58]. Since the norm of $\gamma$ equals the angle of rotation, half of the rotation with $\mathbf{R}_{\boldsymbol{\gamma}}$ is obtained by applying Rodrigues' formula on $\gamma / 2$, which results in $\mathbf{R}_{\gamma / 2}$. The mean rotation matrix is determined by using the result of Rodrigues' formula to rotate the frame of the first node:

$$
\overline{\mathbf{R}}=\mathbf{R}_{\gamma / 2} \mathbf{T}
$$

Three orthogonal vectors can be extracted from the mean rotation matrix: $\overline{\mathbf{R}}=$ $\left[\boldsymbol{r}_{1}, \boldsymbol{r}_{2}, \boldsymbol{r}_{3}\right]$. These are used to determine the remaining components of $\mathbf{E}$ :

$$
e_{2}=\frac{r_{3} \times e_{1}}{\left\|r_{3} \times e_{1}\right\|} \quad e_{3}=e_{1} \times e_{2}
$$

The local rotational degrees of freedom, from here on referred to as local rotations, are the rotations of the nodes with respect to the local element basis. They can 
be determined from the nodal and element frames [15]:

$$
\begin{array}{ll}
2 \sin \psi_{1}=-\boldsymbol{t}_{3}^{\mathrm{T}} \boldsymbol{e}_{2}+\boldsymbol{e}_{3}^{\mathrm{T}} \boldsymbol{t}_{2} & 2 \sin \psi_{2}=-\boldsymbol{u}_{3}^{\mathrm{T}} \boldsymbol{e}_{2}+\boldsymbol{e}_{3}^{\mathrm{T}} \boldsymbol{u}_{2} \\
2 \sin \theta_{1}=\boldsymbol{t}_{3}^{\mathrm{T}} \boldsymbol{e}_{1}-\boldsymbol{e}_{3}^{\mathrm{T}} \boldsymbol{t}_{1} & 2 \sin \theta_{2}=\boldsymbol{u}_{3}^{\mathrm{T}} \boldsymbol{e}_{1}-\boldsymbol{e}_{3}^{\mathrm{T}} \boldsymbol{u}_{1} \\
2 \sin \phi_{1}=-\boldsymbol{t}_{2}^{\mathrm{T}} \boldsymbol{e}_{1}+\boldsymbol{e}_{2}^{\mathrm{T}} \boldsymbol{t}_{1} & 2 \sin \phi_{2}=-\boldsymbol{u}_{2}^{\mathrm{T}} \boldsymbol{e}_{1}+\boldsymbol{e}_{2}^{\mathrm{T}} \boldsymbol{u}_{1}
\end{array}
$$

\subsubsection{Transformation matrix}

The transformation matrix relates the local to the global degrees of freedom. It has size $7 \times 12$ and is derived by taking the variation of the local degrees of freedom expressed by Equation (2.1). Taking the variation of a vector, indicated by $\delta$, means finding the change of that vector by an infinitesimal change in the global degrees of freedom vector $\boldsymbol{p}$ :

$$
\delta \boldsymbol{p}_{\mathrm{L}}=\frac{\partial \boldsymbol{p}_{\mathrm{L}}}{\partial \boldsymbol{p}} \delta \boldsymbol{p}=\mathbf{B} \delta \boldsymbol{p}
$$

The derivation of transformation matrix $\mathbf{B}$ can be found in [15] and results in:

$$
\mathbf{B}=\left[\begin{array}{lllllll}
\frac{\overline{\boldsymbol{b}}_{1}^{\mathrm{T}}}{2 \cos \psi_{1}} & \frac{-\overline{\boldsymbol{b}}_{2}^{\mathrm{T}}}{2 \cos \theta_{1}} & \frac{\overline{\boldsymbol{b}}_{3}^{\mathrm{T}}}{2 \cos \phi_{1}} & \overline{\boldsymbol{b}}_{4}^{\mathrm{T}} & \frac{\overline{\boldsymbol{b}}_{5}^{\mathrm{T}}}{2 \cos \psi_{2}} & \frac{-\overline{\boldsymbol{b}}_{6}^{\mathrm{T}}}{2 \cos \theta_{2}} & \frac{\overline{\boldsymbol{b}}_{7}^{\mathrm{T}}}{2 \cos \phi_{2}}
\end{array}\right]^{\mathrm{T}}
$$

with

$$
\begin{aligned}
& \overline{\boldsymbol{b}}_{1}=\left[\begin{array}{cccc}
\mathbf{0} & \boldsymbol{e}_{2}^{\mathrm{T}} \tilde{\boldsymbol{t}}_{3}-\boldsymbol{e}_{3}^{\mathrm{T}} \tilde{\boldsymbol{t}}_{2} & \mathbf{0} & \mathbf{0}
\end{array}\right]+\boldsymbol{t}_{2}^{\mathrm{T}} \mathbf{Q}_{3}-\boldsymbol{t}_{3}^{\mathrm{T}} \mathbf{Q}_{2} \\
& \overline{\boldsymbol{b}}_{2}=\left[\begin{array}{llll}
\boldsymbol{t}_{3}^{\mathrm{T}} \mathbf{A} & \boldsymbol{e}_{1}^{\mathrm{T}} \tilde{\boldsymbol{t}}_{3}-\boldsymbol{e}_{3}^{\mathrm{T}} \tilde{\boldsymbol{t}}_{1}-\boldsymbol{t}_{3}^{\mathrm{T}} \mathbf{A} & \mathbf{0}
\end{array}\right] \quad \begin{array}{l}
+\boldsymbol{t}_{1}^{\mathrm{T}} \mathbf{Q}_{3} \\
\boldsymbol{t}_{2}^{\mathrm{T}} \mathbf{A}
\end{array} \\
& \overline{\boldsymbol{b}}_{3}=\left[\begin{array}{lll}
\boldsymbol{t}_{2}^{\mathrm{T}} \mathbf{A} & \boldsymbol{e}_{1}^{\mathrm{T}} \tilde{\boldsymbol{t}}_{2}-\boldsymbol{e}_{2}^{\mathrm{T}} \tilde{\boldsymbol{t}}_{1}-\boldsymbol{t}_{2}^{\mathrm{T}} \mathbf{A} \quad \mathbf{0}
\end{array}\right]+\boldsymbol{t}_{1}^{\mathrm{T}} \mathbf{Q}_{2} \\
& \overline{\boldsymbol{b}}_{4}=\left[\begin{array}{cccc}
-e_{1}^{\mathrm{T}} & \mathbf{0} & \boldsymbol{e}_{1}^{\mathrm{T}} & \mathbf{0}
\end{array}\right. \\
& \overline{\boldsymbol{b}}_{5}=\left[\begin{array}{cccc}
\mathbf{0} & \mathbf{0} & \mathbf{0} & \boldsymbol{e}_{2}^{\mathrm{T}} \tilde{\boldsymbol{u}}_{3}-\boldsymbol{e}_{3}^{\mathrm{T}} \tilde{\boldsymbol{u}}_{2}
\end{array}\right]+\boldsymbol{u}_{2}^{\mathrm{T}} \mathbf{Q}_{3}-\boldsymbol{u}_{3}^{\mathrm{T}} \mathbf{Q}_{2} \\
& \overline{\boldsymbol{b}}_{6}=\left[\begin{array}{lll}
\boldsymbol{u}_{3}^{\mathrm{T}} \mathbf{A} & \mathbf{0} & -\boldsymbol{u}_{3}^{\mathrm{T}} \mathbf{A} \boldsymbol{e}_{1}^{\mathrm{T}} \tilde{\boldsymbol{u}}_{3}-\boldsymbol{e}_{3}^{\mathrm{T}} \tilde{\boldsymbol{u}}_{1}
\end{array}\right] \quad+\boldsymbol{u}_{1}^{\mathrm{T}} \mathbf{Q}_{3} \\
& \overline{\boldsymbol{b}}_{7}=\left[\begin{array}{lll}
\boldsymbol{u}_{2}^{\mathrm{T}} \mathbf{A} & \mathbf{0} & -\boldsymbol{u}_{2}^{\mathrm{T}} \mathbf{A} \boldsymbol{e}_{1}^{\mathrm{T}} \tilde{\boldsymbol{u}}_{2}-\boldsymbol{e}_{2}^{\mathrm{T}} \tilde{\boldsymbol{u}}_{1}
\end{array}\right] \quad+\boldsymbol{u}_{1}^{\mathrm{T}} \mathbf{Q}_{2}
\end{aligned}
$$

Here, $\mathbf{0}$ is a $1 \times 3$ zero matrix and $\tilde{\boldsymbol{t}}_{i}$ and $\tilde{\boldsymbol{u}}_{i}$ denote skew symmetric cross product matrices of $\boldsymbol{t}_{i}$ and $\boldsymbol{u}_{i}$, such as:

$$
\tilde{\boldsymbol{t}}_{i}=\left[\begin{array}{ccc}
0 & -t_{i 3} & t_{i 2} \\
t_{i 3} & 0 & -t_{i 1} \\
-t_{i 2} & t_{i 1} & 0
\end{array}\right]
$$

Matrix A follows from the variation of $\boldsymbol{e}_{1}$ :

$$
\delta \boldsymbol{e}_{1}=\mathbf{A} \delta \boldsymbol{d}_{21} \quad \text { with } \quad \mathbf{A}=\frac{1}{L}\left(\mathbf{I}-\boldsymbol{e}_{1} \boldsymbol{e}_{1}^{\mathrm{T}}\right)
$$

Here, $\mathbf{I}$ is the identity matrix. $\mathbf{Q}_{2}$ and $\mathbf{Q}_{3}$ are $3 \times 12$ matrices that result from the variation $\delta \boldsymbol{e}_{2}$ and $\delta \boldsymbol{e}_{3}$. With ancillary vector $\overline{\boldsymbol{e}}_{2}=\boldsymbol{r}_{3} \times \boldsymbol{e}_{1}$, these variations and matrices are:

$$
\begin{array}{llll}
\delta \boldsymbol{e}_{2}=\mathbf{Q}_{2} \delta \boldsymbol{p} & \text { with } & \mathbf{Q}_{2}=\frac{\mathbf{I}-\overline{\boldsymbol{e}}_{2} \overline{\boldsymbol{e}}_{2}^{\mathrm{T}}}{\left\|\overline{\boldsymbol{e}}_{2}\right\|}\left[\begin{array}{lllll}
-\tilde{\boldsymbol{r}}_{3} \mathbf{A} & \frac{1}{2} \tilde{\boldsymbol{e}}_{1} \tilde{\boldsymbol{r}}_{3} & \tilde{\boldsymbol{r}}_{3} \mathbf{A} & \frac{1}{2} \tilde{\boldsymbol{e}}_{1} \tilde{\boldsymbol{r}}_{3}
\end{array}\right] \\
\delta \boldsymbol{e}_{3}=\mathbf{Q}_{3} \delta \boldsymbol{p} & \text { with } & \mathbf{Q}_{3}=\left[\begin{array}{lllll}
\tilde{\boldsymbol{e}}_{2} \mathbf{A} & \mathbf{0} & -\tilde{\boldsymbol{e}}_{2} \mathbf{A} & \mathbf{0}
\end{array}\right]+\tilde{\boldsymbol{e}}_{1} \mathbf{Q}_{2}
\end{array}
$$




\subsubsection{Rotation matrix}

In the element formulation of Crisfield the transformation matrix is used to transform the forces from the local to the global coordinate system:

$$
\boldsymbol{F}=\mathbf{B}^{\mathrm{T}} \boldsymbol{F}_{\mathrm{L}}^{7}
$$

Here, $\boldsymbol{F}$ is a vector of length 12 with forces and moments in the global coordinate system and $\boldsymbol{F}_{\mathrm{L}}^{7}$ is a vector of length 7 with forces and moments in the local coordinate system, that correspond to the 7 local degrees of freedom in Equation (2.1).

The transformation matrix used in the original element formulation of Crisfield consists of several sub-matrices, which are functions of the nodal and element frame, as can be seen in Equations (2.13-2.17). The geometric stiffness matrix is determined from the derivatives of all these sub-matrices to the global degrees of freedom, which makes the calculation of the geometric stiffness matrix computationally expensive. To reduce computational costs a less complicated rotation matrix is proposed, from which an inexpensive geometric stiffness matrix can be derived. As in the formulation of Crisfield, it is assumed that local rotations are small. By using the same assumption, rotating the forces with the new rotation matrix does not compromise on accuracy. This section presents the new rotation matrix.

The element forces and moments are determined in the local coordinate system, which is represented by element frame $\mathbf{E}$. This frame can also be used to rotate forces and moments expressed in the local coordinate system to the global coordinate system. Battini and Pacoste [6], who use a two-step rotation of the element forces and moments, pointed out that a direct rotation of element moments results in an incorrect prediction of the displacements when torsional moments are involved. Therefore, rotating the element moments requires a different approach.

In Figure 2.3 the nodal moments of two adjacent elements are shown in their respective local coordinate systems. It can be seen that the directions of these moments are relative to element frame $\mathbf{E}$. In the nodal summation of the moments the torsional moment of an element is in equilibrium with the torsional moment and the bending moments of the adjacent element. Therefore, a transfer occurs between the torsional moment and the bending moments. In Figure 2.4 the nodal moments of two adjacent elements are shown in the coordinate system of their common node. When these moments are summed, the torsional moment of an element is in equilibrium with the torsional moment of the adjacent element. Each bending moment is in equilibrium with its respective bending moment of the adjacent element.

The representation in Figure 2.4 can be achieved assuming that local rotations are small, from which it follows that the magnitudes of the moments in frames $\mathbf{T}$ and $\mathbf{U}$ are approximately equal to those in frame $\mathbf{E}$. The obtained moments are rotated 


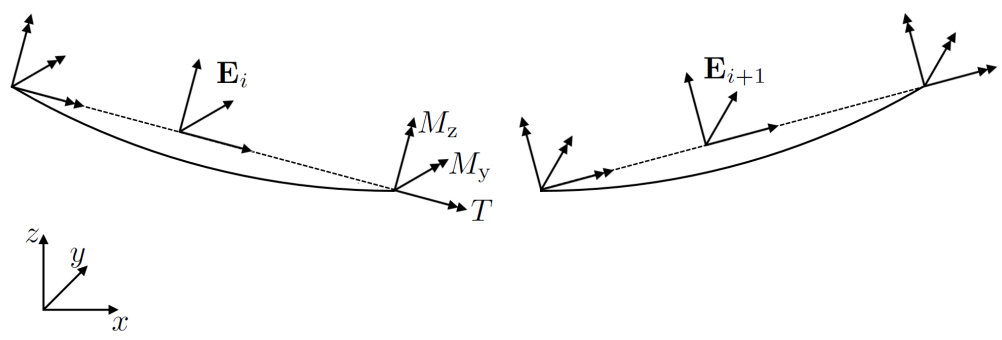

Figure 2.3: Nodal moments represented in element frames $\mathbf{E}$ of two adjacent elements.
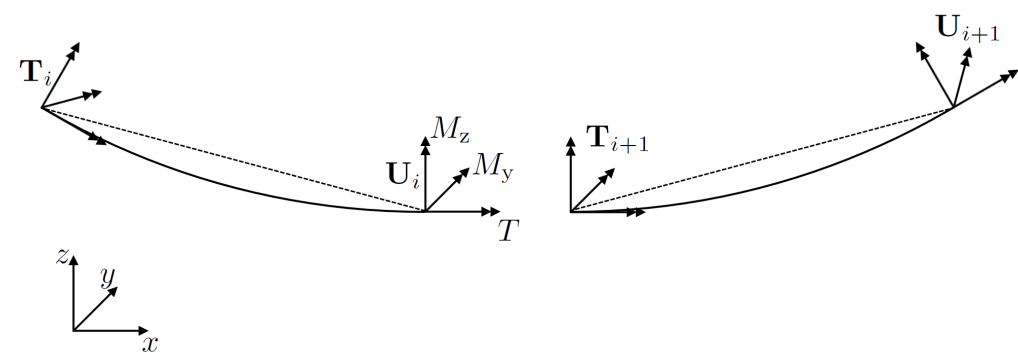

Figure 2.4: Nodal moments represented in the nodal frame that two adjacent elements have in common.

to the global coordinate system using frames $\mathbf{T}$ and $\mathbf{U}$. In Section 2.2 .2 it will be shown that when a rotation matrix with the nodal frames to rotate moments is used more accurate results are obtained for problems that involve torsion. With element frame $\mathbf{E}$ to rotate the forces and nodal frames $\mathbf{T}$ and $\mathbf{U}$ to rotate the moments, the rotation matrix is:

$$
\mathbf{R}=\left[\begin{array}{llll}
\mathbf{E}^{\mathrm{T}} & & & \\
& \mathbf{T}^{\mathrm{T}} & & \\
& & \mathbf{E}^{\mathrm{T}} & \\
& & & \mathbf{U}^{\mathrm{T}}
\end{array}\right]
$$

The new rotation matrix $\mathbf{R}$ rotates the local element forces and moments to the global coordinate system:

$$
\boldsymbol{F}=\mathbf{R}^{\mathrm{T}} \boldsymbol{F}_{\mathrm{L}}
$$

This matrix is of size $12 \times 12$ and the force vectors have length 12 . The individual elements of the force vectors can be seen in Figure 2.5. In the force vectors, they 
are ordered as:

$$
\begin{aligned}
& \boldsymbol{F}_{\mathrm{L}}=\left[\begin{array}{llllllllllll}
N_{1} & F_{\mathrm{y} 1} & F_{\mathrm{z} 1} & T_{1} & M_{\mathrm{y} 1} & M_{\mathrm{z} 1} & N_{2} & F_{\mathrm{y} 2} & F_{\mathrm{z} 2} & T_{2} & M_{\mathrm{y} 2} & M_{\mathrm{z} 2}
\end{array}\right]^{\mathrm{T}} \\
& \boldsymbol{F}=\left[\begin{array}{llllllllllll}
F_{\mathrm{x} 1}^{\mathrm{g}} & F_{\mathrm{y} 1}^{\mathrm{g}} & F_{\mathrm{z} 1}^{\mathrm{g}} & M_{\mathrm{x} 1}^{\mathrm{g}} & M_{\mathrm{y} 1}^{\mathrm{g}} & M_{\mathrm{z} 1}^{\mathrm{g}} & F_{\mathrm{x} 2}^{\mathrm{g}} & F_{\mathrm{y} 2}^{\mathrm{g}} & F_{\mathrm{z} 2}^{\mathrm{g}} & M_{\mathrm{x} 2}^{\mathrm{g}} & M_{\mathrm{y} 2}^{\mathrm{g}} & M_{\mathrm{z} 2}^{\mathrm{g}}
\end{array}\right]^{\mathrm{T}}
\end{aligned}
$$

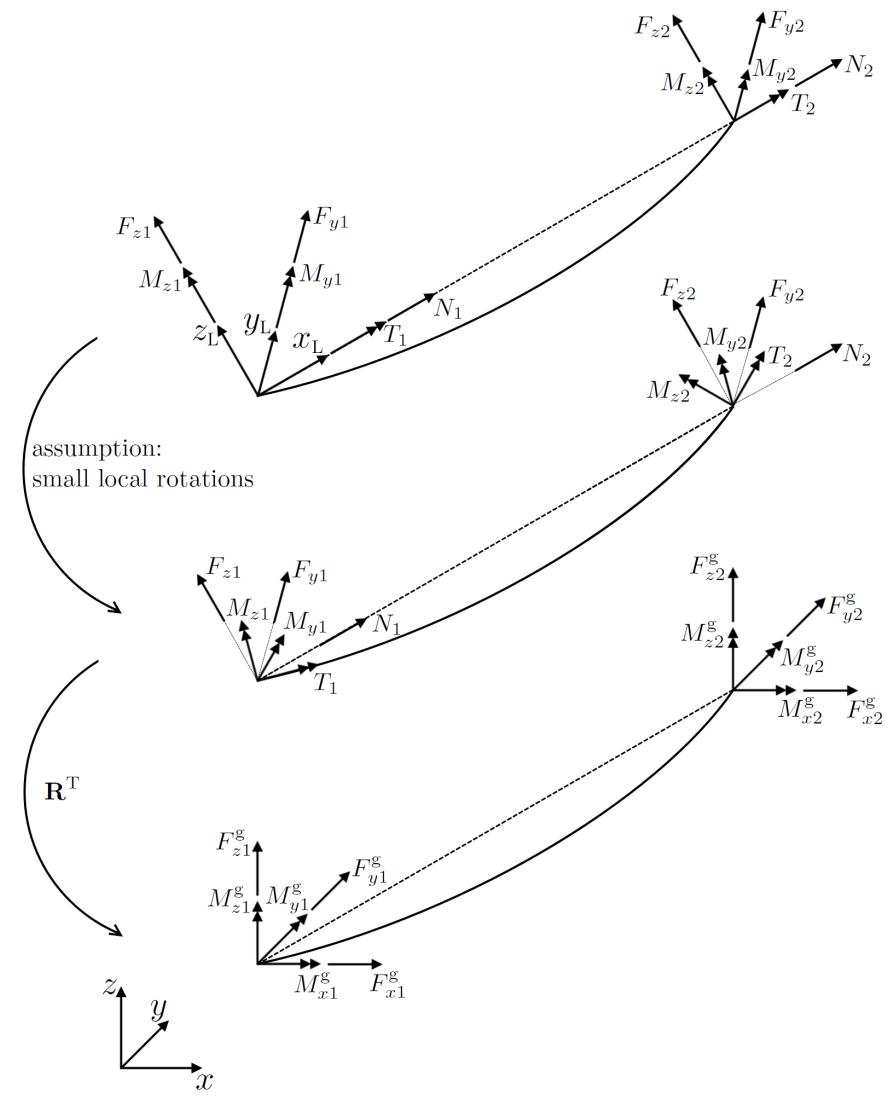

Figure 2.5: The forces are determined in the local coordinate system and rotated to the global coordinate system.

Numerical experiments were performed to see whether the nodal frames $\mathbf{T}$ and $\mathbf{U}$ can also be used to rotate the nodal forces. In these experiments the NewtonRaphson algorithm did not converge. A plausible cause of this convergence issue is that derivatives of the nodal frames only contain rotational terms, thus in this case the geometric stiffness matrix only has rotational terms. This means that, for example, an increased bending stiffness due to a positive axial force is not represented in the geometric stiffness matrix. 


\subsubsection{Local material stiffness}

The local element forces can be obtained from the local variables using local stiffness matrix $\mathbf{K}_{\mathrm{L}}$. The local displacement vector has length 7 , as there are seven local degrees of freedom. The local force vector has length 12 , hence a stiffness matrix of size $12 \times 7$ is required:

$$
\boldsymbol{F}_{\mathrm{L}}^{12}=\mathbf{K}_{\mathrm{L}}^{12 \times 7} \boldsymbol{p}_{\mathrm{L}}^{7}
$$

For beam elements, a material stiffness matrix can be determined with an integral over the element's length:

$$
\mathbf{K}_{\mathrm{L}}=\int_{0}^{L_{0}} \mathbf{C}^{\mathrm{T}} \mathbf{D C} d x
$$

Here, $\mathbf{D}$ is a diagonal matrix that relates the elements internal forces and moment to its strains and curvatures:

$$
\mathbf{D}=\operatorname{diag}\left(E A, G J, E I_{\mathrm{y}}, E I_{\mathrm{z}}\right)
$$

Matrix $\mathbf{C}$ contains derivatives of vectors with interpolation functions $\boldsymbol{N}_{i}$ :

$$
\mathbf{C}(x)=\left[\begin{array}{llll}
\frac{d \boldsymbol{N}_{\mathrm{u}}}{d x} & \frac{d \boldsymbol{N}_{\psi}}{d x} & \frac{d^{2} \boldsymbol{N}_{\mathrm{w}}}{d x^{2}} & \frac{d^{2} \boldsymbol{N}_{\mathrm{v}}}{d x^{2}}
\end{array}\right]^{\mathrm{T}}
$$

There are four vectors $\boldsymbol{N}_{i}$ : three for displacements in $x_{\mathrm{L}^{-}}, y_{\mathrm{L}^{-}}$and $z_{\mathrm{L}}$-direction, and one for torsional rotation about the $x_{\mathrm{L}}$-axis. The rotations about the $y_{\mathrm{L}^{-}}$and $z_{\mathrm{L}}$-axis do not have individual interpolation functions, as they can be determined from the derivative of displacements in $z_{\mathrm{L}}$ - and $y_{\mathrm{L}}$-direction.

In this work two linear $\left(l_{i}\right)$ and four Hermite $\left(h_{i}\right)$ interpolation functions are chosen. These are functions of a local coordinate $\xi$, which is 0 at the first node and 1 at the second node.

$$
\begin{array}{ll}
l_{1}=1-\xi & l_{2}=\xi \\
h_{1}=2 \xi^{3}-3 \xi^{2}+1 & h_{2}=\xi^{3}-2 \xi^{2}+\xi \\
h_{3}=-2 \xi^{3}+3 \xi^{2} & h_{4}=\xi^{3}-\xi^{2}
\end{array}
$$

Each interpolation function corresponds to a local degree of freedom. When twelve local degrees of freedom are involved, the vectors $\boldsymbol{N}_{i}$ and their corresponding degrees of freedom are:

$$
\begin{aligned}
& \begin{array}{llllllllllll}
u_{1} & v_{1} & w_{1} & \psi_{1} & \theta_{1} & \phi_{1} & u_{2} & v_{2} & w_{2} & \psi_{2} & \theta_{2} & \phi_{2}
\end{array}
\end{aligned}
$$

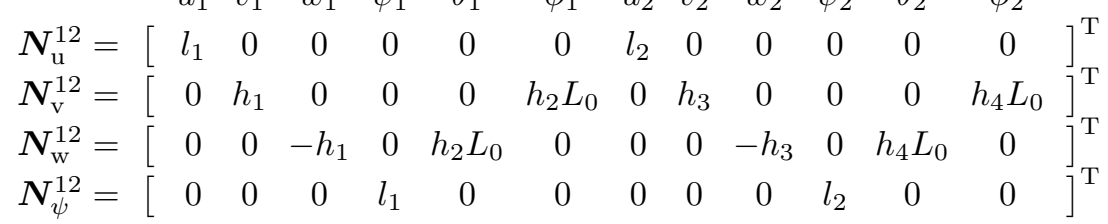


Substituting these vectors $\boldsymbol{N}_{i}$ in Equation (2.25) results in a $4 \times 12$ matrix:

$$
\mathbf{C}^{12}(x)=\left[\begin{array}{llll}
\frac{d \boldsymbol{N}_{\mathrm{u}}^{12}}{d x} & \frac{d \boldsymbol{N}_{\psi}^{12}}{d x} & \frac{d^{2} \boldsymbol{N}_{\mathrm{w}}^{12}}{d x^{2}} & \frac{d^{2} \boldsymbol{N}_{\mathrm{v}}^{12}}{d x^{2}}
\end{array}\right]^{\mathrm{T}}
$$

Using this matrix to evaluate Equation (2.23) leads to a stiffness matrix of size $12 \times 12$, corresponding to twelve local degrees of freedom.

Because five of the local degrees of freedom are always zero, the number of local degrees of freedom is reduced to seven. This reduces Equation (2.27) to:

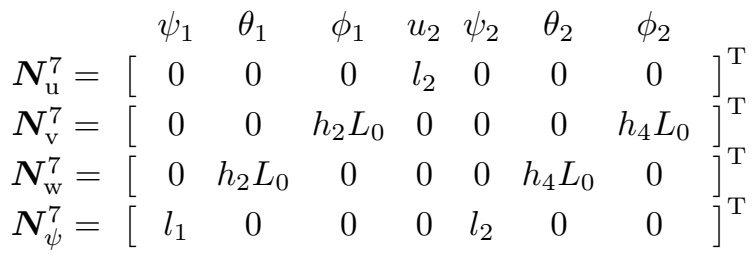

Substituting these vectors $\boldsymbol{N}_{i}$ in Equation (2.25) results in a $4 \times 7$ matrix:

$$
\mathbf{C}^{7}(x)=\left[\begin{array}{llll}
\frac{d \boldsymbol{N}_{\mathrm{u}}^{7}}{d x} & \frac{d \boldsymbol{N}_{\psi}^{7}}{d x} & \frac{d^{2} \boldsymbol{N}_{\mathrm{w}}^{7}}{d x^{2}} & \frac{d^{2} \boldsymbol{N}_{\mathrm{v}}^{7}}{d x^{2}}
\end{array}\right]^{\mathrm{T}}
$$

Using this matrix to evaluate Equation (2.23) leads to a stiffness matrix of size $7 \times 7$, as used in the element formulations of Crisfield and Battini-Pacoste.

The stiffness matrix of size $12 \times 7$ is determined by evaluating Equation (2.23) with both $\mathbf{C}^{12}$ and $\mathbf{C}^{7}$ from Equations (2.28) and (2.30) respectively:

$$
\mathbf{K}_{\mathrm{L}}^{12 \times 7}=\int_{0}^{L_{0}}\left(\mathbf{C}^{12}\right)^{\mathrm{T}} \mathbf{D} \mathbf{C}^{7} d x
$$

This stiffness matrix is constant and only needs to be computed once. In the next section it will be shown that this matrix must be rotated with its corresponding element.

\subsubsection{Global stiffness matrix}

The global stiffness matrix is determined by taking the variation of the force vector in Equation (2.20) with respect to the global degrees of freedom:

$$
\delta \boldsymbol{F}=\mathbf{R}^{\mathrm{T}} \delta \boldsymbol{F}_{\mathrm{L}}+\delta \mathbf{R}^{\mathrm{T}} \boldsymbol{F}_{\mathrm{L}}
$$

The first term in Equation (2.32) leads to the material stiffness matrix, which can be seen as the change in the local element forces due to a change in the global degrees of freedom, while the rotation matrix remains constant. The second term in Equation (2.32) leads to the geometric stiffness matrix, which can be seen as the change in the rotation matrix due to a change in the global degrees of freedom, 
while the local force vector remains constant. Substituting the variation of the local force vector $\delta \boldsymbol{f}_{\mathrm{L}}=\mathbf{K}_{\mathrm{L}} \delta \boldsymbol{p}_{\mathrm{L}}$ and the variation of the local degrees of freedom vector $\delta \boldsymbol{p}_{\mathrm{L}}=\mathbf{B} \delta \boldsymbol{p}$ yields:

$$
\delta \boldsymbol{F}=\mathbf{R}^{\mathrm{T}} \mathbf{K}_{\mathrm{L}} \mathbf{B} \delta \boldsymbol{p}+\delta \mathbf{R}^{\mathrm{T}} \boldsymbol{F}_{\mathrm{L}}
$$

The first term of Equation (2.33) results in the material stiffness matrix:

$$
\mathbf{K}_{\text {mat }}=\mathbf{R}^{\mathrm{T}} \mathbf{K}_{\mathrm{L}} \mathbf{B}
$$

It can be seen that the material stiffness matrix is determined by rotating the local material stiffness matrix from Section 2.1.4. In this rotation both the new rotation matrix $\mathbf{R}$ and the transformation matrix $\mathbf{B}$ from the original element formulation of Crisfield are used.

The geometric stiffness matrix is determined by expanding the second term of Equation (2.33). The variation $\delta \mathbf{R}^{\mathrm{T}}$ is determined from the variations of frames $\mathbf{T}, \mathbf{E}$ or $\mathbf{U}$ :

$$
\delta \mathbf{R}^{\mathrm{T}} \boldsymbol{F}_{\mathrm{L}}=\left[\begin{array}{cccc}
\delta \mathbf{E} & \mathbf{0} & \mathbf{0} & \mathbf{0} \\
\mathbf{0} & \delta \mathbf{T} & \mathbf{0} & \mathbf{0} \\
\mathbf{0} & \mathbf{0} & \delta \mathbf{E} & \mathbf{0} \\
\mathbf{0} & \mathbf{0} & \mathbf{0} & \delta \mathbf{U}
\end{array}\right] \boldsymbol{F}_{\mathrm{L}}
$$

Here, and in the remainder of this section, $\mathbf{0}$ is a zero matrix of size $3 \times 3$, unless a different size is indicated. Each column of $\delta \mathbf{R}^{\mathrm{T}}$ contains the variation of one vector from frames $\mathbf{T}, \mathbf{E}$ or $\mathbf{U}$. The variation of the vectors in frame $\mathbf{E}$ are shown in Equations (2.16) and (2.17). The variation of the vectors in $\mathbf{T}$ and $\mathbf{U}$ are:

$$
\delta \boldsymbol{t}_{i}=-\tilde{\boldsymbol{t}}_{i} \delta \Delta \boldsymbol{\alpha}_{1} \quad \delta \boldsymbol{u}_{i}=-\tilde{\boldsymbol{u}}_{i} \delta \Delta \boldsymbol{\alpha}_{2}
$$

Here, $\delta \Delta \boldsymbol{\alpha}_{1}$ and $\delta \Delta \boldsymbol{\alpha}_{2}$ are variations of the rotational components of the global degrees of freedom vector $\boldsymbol{p}$, which can be seen in Equation (2.6). Using these variations, a matrix multiplied with the variation of $\boldsymbol{p}$ can be determined from each individual column of Equation (2.35). For example, the first column results in:

$$
\delta \mathbf{R}_{1}^{\mathrm{T}} N_{1}=N_{1}\left[\begin{array}{c}
\delta \boldsymbol{e}_{1} \\
\mathbf{0}^{1 \times 3} \\
\mathbf{0}^{1 \times 3} \\
\mathbf{0}^{1 \times 3}
\end{array}\right]=N_{1}\left[\begin{array}{cccc}
-\mathbf{A} & \mathbf{0} & \mathbf{A} & \mathbf{0} \\
\mathbf{0} & \mathbf{0} & \mathbf{0} & \mathbf{0} \\
\mathbf{0} & \mathbf{0} & \mathbf{0} & \mathbf{0} \\
\mathbf{0} & \mathbf{0} & \mathbf{0} & \mathbf{0}
\end{array}\right] \delta \boldsymbol{p}
$$

Here, $\mathbf{A}$ is a matrix of size $3 \times 3$ as defined in Equation (2.16). The geometric stiffness matrix is determined by summing the results from each individual column of Equation (2.35):

$$
\mathbf{K}_{\text {geo }}=\left[\begin{array}{cccc}
-N_{1} \mathbf{A} & \mathbf{0} & N_{1} \mathbf{A} & \mathbf{0} \\
\mathbf{0} & \mathbf{G}_{1} & \mathbf{0} & \mathbf{0} \\
-N_{2} \mathbf{A} & \mathbf{0} & N_{2} \mathbf{A} & \mathbf{0} \\
\mathbf{0} & \mathbf{0} & \mathbf{0} & \mathbf{G}_{2}
\end{array}\right]+\left[\begin{array}{c}
F_{\mathrm{y} 1} \mathbf{Q}_{2}+F_{\mathrm{z} 1} \mathbf{Q}_{3} \\
\mathbf{0}^{3 \times 12} \\
F_{\mathrm{y} 2} \mathbf{Q}_{2}+F_{\mathrm{z} 2} \mathbf{Q}_{3} \\
\mathbf{0}^{3 \times 12}
\end{array}\right]
$$


where the terms following from the variation of $\mathbf{T}$ and $\mathbf{U}$ are:

$$
\mathbf{G}_{1}=-T_{1} \tilde{\boldsymbol{t}}_{1}-M_{\mathrm{y} 1} \tilde{\boldsymbol{t}}_{2}-M_{\mathrm{z} 1} \tilde{\boldsymbol{t}}_{3} \quad \mathbf{G}_{2}=-T_{2} \tilde{\boldsymbol{u}}_{1}-M_{\mathrm{y} 2} \tilde{\boldsymbol{u}}_{2}-M_{\mathrm{z} 2} \tilde{\boldsymbol{u}}_{3} .
$$

All sub-matrices of this geometric stiffness matrix have already been evaluated in the computation of the transformation matrix. Therefore, computing this geometric stiffness matrix is inexpensive.

The tangent stiffness matrix $\mathbf{K}=\mathbf{K}_{\text {mat }}+\mathbf{K}_{\text {geo }}$ is generally asymmetric, similar to the formulations of Crisfield and Battini-Pacoste. For both formulations the asymmetric part of the stiffness matrix becomes small when the Newton-Raphson algorithm has converged, except for problems where a concentrated moment is applied on the structure [6]. Apart from this exception, the matrix can be symmetrised without losing quadratic convergence in the Newton-Raphson algorithm.

Numerical comparisons show that the Frobenius norm of the asymmetrical part of the tangent stiffness matrix in the new formulation is smaller than that in the formulation of Crisfield:

$$
\frac{1}{2}\left\|\mathbf{K}-\mathbf{K}^{\mathrm{T}}\right\|<\frac{1}{2}\left\|\mathbf{K}_{\mathrm{C}}-\mathbf{K}_{\mathrm{C}}^{\mathrm{T}}\right\|
$$

It can be concluded that, in the new formulation, the stiffness matrix can be symmetrised without losing quadratic convergence in the Newton-Raphson algorithm, except for problems where a concentrated moment is applied on the structure. Numerical experiments with a symmetrised tangent stiffness matrix support this conclusion.

However, symmetrising the stiffness matrix does not significantly decrease computational time. The system of equations that needs to be solved in the Newton-Raphson algorithm involves a sparse matrix that only has terms on and near the diagonal. Solving this system is computational inexpensive, regardless of whether the matrix is symmetric or not. These computational costs are insignificant in comparison with the total costs of each element formulation. Therefore, the reduction in computational time of the element formulation when symmetrising the tangent stiffness matrix is insignificant.

\subsection{Comparison of element formulations}

The new element formulation is compared to the formulations of Crisfield and Battini-Pacoste, by an implementation of these formulations in Matlab [37]. The formulation of Crisfield is implemented as in [15], the formulation of BattiniPacoste is implemented as in [5]. To get a fair comparison in computation time, a small adjustment is made to the formulation of Battini-Pacoste. This formulation originally uses the exponential and logarithm of a matrix, but the standard Matlab functions for this have high computational costs. The exponential and logarithm of 
a matrix are replaced with, respectively, Rodrigues' rotation matrix and Spurrier's algorithm, which give the same results and are computationally less expensive.

The three element formulations will be compared in two examples. In the first example a pipe is bent to form a two-dimensional circle, in the second example a beam is loaded to form a three-dimensional helix.

\subsubsection{Example 1: Two-dimensional circle}

The example of a two-dimensional circle is taken from [13]; here it is implemented as a pipe with properties shown in Table 2.1. The pipe is fixed on one end and loaded with a moment on the other end. This moment is applied in ten equal increments, until the pipe forms a circle. Based on curvature, the total applied moment to form a closed circle is equal to:

$$
M=\frac{E I 2 \pi}{L}
$$

Table 2.1: Pipe properties.

\begin{tabular}{lll}
\hline Length & $L$ & $100 \mathrm{~m}$ \\
Diameter & $D$ & $457 \mathrm{~mm}(18 \mathrm{inch})$ \\
Wall Thickness & $t$ & $30.8 \mathrm{~mm}$ \\
Young's modulus & $E$ & $207 \mathrm{GPa}$ \\
Poisson's ratio & $\nu$ & 0.3
\end{tabular}

In this example the pipe consists of ten elements. The initial and deformed shape of the pipe are shown in Figure 2.6a. It can be seen that the initial shape of the pipe is horizontal and straight and that the deformed shape of the pipe is a circle.

Results obtained with the new element formulation are compared to those obtained with the formulations of Crisfield and Battini-Pacoste by the total displacement at each node. The difference in total displacement with the formulation of Crisfield is determined by:

$$
\Delta_{\mathrm{C}}=\frac{\left|\boldsymbol{d}-\boldsymbol{d}_{\mathrm{C}}\right|}{\left|\boldsymbol{d}_{\mathrm{C}}\right|}
$$

Here, $\boldsymbol{d}$ and $\boldsymbol{d}_{\mathrm{C}}$ are vectors with the displacements of all nodes obtained with the new formulation and the formulation of Crisfield respectively. The difference from results obtained with the formulation of Battini-Pacoste, $\Delta_{\mathrm{BP}}$, is determined similarly. Figure $2.6 \mathrm{~b}$ shows the logarithm of $\Delta_{\mathrm{C}}$ and $\Delta_{\mathrm{BP}}$ versus $s$, which is a coordinate along the length of the pipe. It can be seen that the differences between the results are very small.

In all three element formulations the local deformation model does not account for the correct length due to an element's curvature. Therefore, all three formulations 


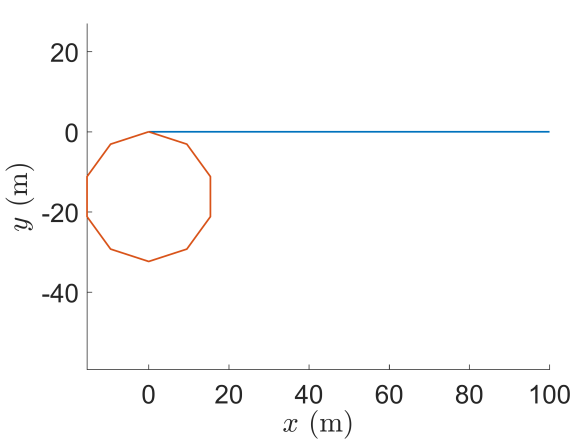

(a) Initial and deformed shape of the $100 \mathrm{~m}$ long pipe.

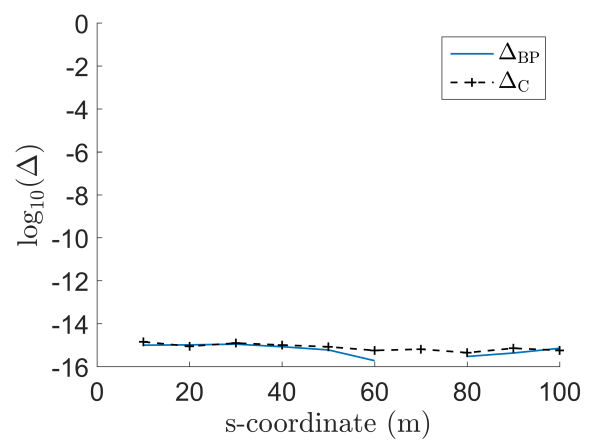

(b) The differences between results obtained with the new element formulation and those obtained with the formulations of Battini-Pacoste and Crisfield are small.

Figure 2.6: Example of a pipe bent in the shape of a circle.

overestimate the radius of the circle. This is not relevant for this comparison, because the local deformation models are equal; to include the correct length would be an identical change for each formulation.

Table 2.2 shows the calculation time of the example for all three formulations. The formulation of Battini-Pacoste is already much faster than that of Crisfield. The new formulation calculates the results of this example $30 \%$ faster than that of Battini-Pacoste.

Table 2.2: Calculation time and total number of iterations.

\begin{tabular}{llllll}
\hline \multirow{2}{*}{ Element formulation } & \multicolumn{2}{c}{$2 \mathrm{D}$ circle } & & \multicolumn{2}{c}{ Helical beam } \\
\cline { 2 - 3 } \cline { 5 - 6 } & Calc time & Iterations & & Calc time & Iterations \\
\hline Crisfield & $0.674 \mathrm{~s}$ & 60 & & $234.4 \mathrm{~s}$ & 3993 \\
Battini-Pacoste & $0.171 \mathrm{~s}$ & 60 & & $70.9 \mathrm{~s}$ & 4084 \\
New formulation & $0.121 \mathrm{~s}$ & 60 & & $53.8 \mathrm{~s}$ & 3960 \\
\hline
\end{tabular}

\subsubsection{Example 2: Helical beam}

The example of the helical beam, which involves a torsional load, was devised by Ibrahimbegović [32] and later adopted by Battini [5]. Here it is used to compare computational time of the considered co-rotational elements and to demonstrate accuracy of the element formulations when torsion moments are involved.

The beam is fixed on one end and on the free end it is loaded with a moment 
about the $y$-axis and a force in $y$-direction. The force and moment are increased proportionally up to their maximum values, which are $F_{\mathrm{y}}=30 \mathrm{~N}$ for the applied force and $M_{\mathrm{y}}=120 \pi \mathrm{Nm}$ for the maximum applied moment. The moment is chosen such that the beam 'rolls up' six times, hence it is equal to six times the moment in Equation (2.41). The applied force and moment are increased proportionally in 1000 steps and the beam is discretised into 60 elements. Properties of the beam are shown in Table 2.3 and the final shape of the helical beam can be seen in Figure 2.7a. This example is identical to the helical beam example in [5].

Table 2.3: Beam properties.

\begin{tabular}{lll}
\hline Length & $L$ & $10 \mathrm{~m}$ \\
Axial rigidity & $E A$ & $100 \mathrm{kN}$ \\
Bending rigidity & $E I$ & $100 \mathrm{Nm}^{2}$ \\
Torsional rigidity & $G J$ & $100 \mathrm{Nm}^{2}$ \\
\hline
\end{tabular}

Because the loads are applied in the global coordinate system, the applied moment is partially a torsion load when the displacement in $y$-direction of the free end is non-zero. Due to this torsional load the displacement of the free end in the direction of the applied force oscillates around zero during the proportional increase of the loads. This can be seen in Figure 2.7b, where the displacement of the free end in $y$-direction is plotted versus a pseudo-time. The domain of the pseudo-time is 0 to 6 , referring to the number of times the beam is rolled up about the $y$-axis.

Figure 2.7b is created using the element formulations of Crisfield, Battini-Pacoste and the new formulation. Results obtained with the formulation of Battini-Pacoste are expected to be accurate, as it uses a two-step algorithm to calculate the transformation and stiffness matrices to account for the difference between nodal and element rotations. It can be seen that the results obtained with the new formulation are similar to those obtained with the formulation of Battini-Pacoste. Up to a pseudo-time of 1.5 the result of the formulation of Crisfield is also similar, but for a higher pseudo-time it is different. When the number of elements is increased, the solution of the formulation of Crisfield converges towards the solutions of Battini-Pacoste and the new formulation, from which it can be concluded that the formulation of Crisfield is inaccurate when large torsional moments are involved.

In Table 2.4 the logarithm of an error norm is shown for each iteration in a representative increment. This error norm is defined by the change in displacement in one iteration as ratio of the total increment. It can be seen that, for all three formulations, the logarithm of the error norm in iteration 4 is more than twice that of iteration 3. All three formulations result in quadratic convergence in the Newton-Raphson algorithm. 


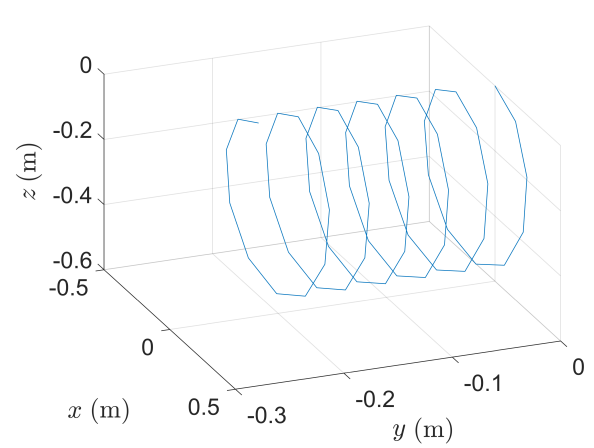

(a) Final shape of the beam obtained with the formulation of Battini-Pacoste.

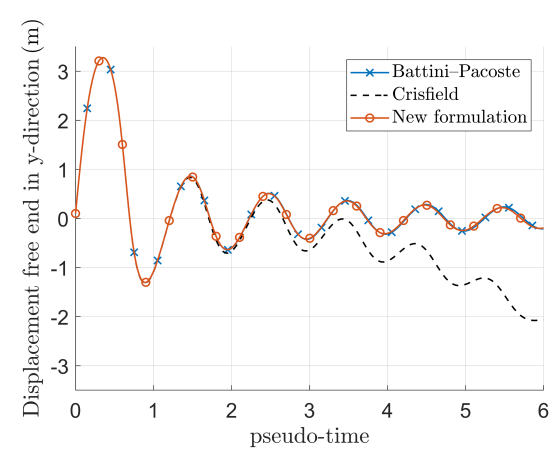

(b) The element formulation of Crisfield becomes inaccurate when the pseudo-time increases.

Figure 2.7: Results of the helical beam example.

Table 2.4: Convergence of the Newton-Raphson algorithm in a representative increment.

\begin{tabular}{llllll}
\hline Iteration number & 1 & 2 & 3 & \multicolumn{1}{l}{4} & 5 \\
\hline Crisfield & -0.30 & -2.52 & -4.01 & -9.94 & -14.88 \\
Battini-Pacoste & -0.30 & -2.52 & -4.01 & -9.95 & -14.19 \\
New formulation & -0.30 & -2.52 & -5.58 & -12.84 & \\
\hline
\end{tabular}

The computational costs of the different element formulations are shown in Table 2.2. Just as for the two-dimensional example, the formulation of Battini-Pacoste is computationally less expensive than that of Crisfield. The new formulation has the lowest computational cost, showing that the new formulation is the most efficient, also for this example.

When fewer elements are used in this example, local rotations become large. Of the three element formulations considered in this chapter, only that of Battini-Pacoste is derived without the assumption of small local rotations. Therefore, only the formulation of Battini-Pacoste is expected to remain accurate when fewer elements are used. The helical beam example is repeated with 30 elements instead of 60 elements, which means that there are only five elements per 'circle' at pseudo-time 6. The resulting displacements of the free end in $y$-direction are shown in Figure 2.8. It can be seen that when using the formulation of Crisfield, the result deviates significantly from the correct solution. To a lesser extend, results obtained with the new formulation and the formulation of Battini-Pacoste also deviate from the solution obtained with 60 elements. The result obtained with the formulation of Battini-Pacoste deviates slightly less than that obtained with the new formulation. 


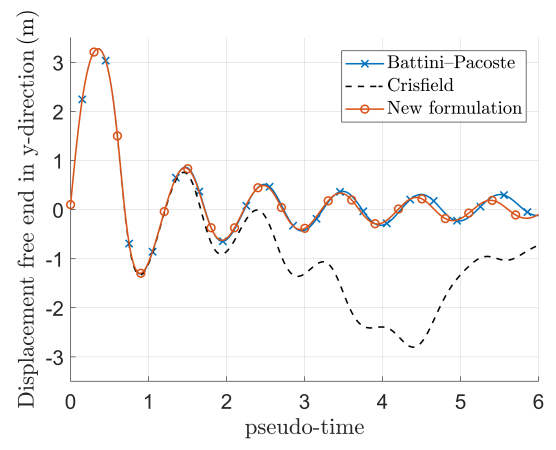

Figure 2.8: Results of the helical beam example with 30 elements.

\subsection{Newton-Raphson algorithm}

In a static pipe-lay simulation the structure is in static equilibrium. In equation form this is represented by a force balance between an internal and an external force vector:

$$
\boldsymbol{F}^{\mathrm{int}}(\Delta \boldsymbol{d})=\boldsymbol{F}^{\mathrm{ext}}(\Delta \boldsymbol{d})
$$

The internal force vector $\boldsymbol{F}^{\text {int }}$ consists of the internal element forces, which were presented in Section 2.1, and contact forces, which will be presented in Chapter 4. The external force vector $\boldsymbol{F}^{\text {ext }}$ consists of concentrated loads and distributed loads, which are the topic of Section 2.4. When the pipe is submerged the external force vector also contains hydrostatic loads, which will be derived in Section 5.1. $\Delta \boldsymbol{d}$ is the generalised displacement increment, containing both rotations and displacements of the pipe.

Both the internal and external force vectors are nonlinear functions of the displacement increment, therefore an iterative method is required to solve the displacement increment from Equation (2.43). In this work the Newton-Raphson algorithm [43] is used.

The goal of this algorithm is to determine a displacement increment $\Delta \boldsymbol{d}$ at which a residual $\boldsymbol{R}$ is equal to zero. This residual is a nonlinear function of the displacement increment $\boldsymbol{\Delta} \boldsymbol{d}$ and it is obtained by rearranging Equation (2.43):

$$
\boldsymbol{R}=\boldsymbol{F}^{\mathrm{int}}-\boldsymbol{F}^{\mathrm{ext}}=\mathbf{0}
$$

The displacement increment is determined iteratively, starting with an initial guess. This initial guess is a zero vector, such that the total displacements are equal to the result at the end of the previous increment. At the end of iteration $i$, the displacement increment is updated by the change of the displacement increment $\delta \boldsymbol{d}$ :

$$
\Delta \boldsymbol{d}_{i+1}=\Delta \boldsymbol{d}_{i}+\delta \boldsymbol{d}
$$


The residual is not equal to zero at the start of an increment. The iterative change of the displacement increment can be determined from the linearisation of the residual:

$$
\boldsymbol{R}_{i}+\frac{\partial \boldsymbol{R}_{i}}{\partial \Delta \boldsymbol{d}} \delta \boldsymbol{d}=\mathbf{0}
$$

Here, $\frac{\partial \boldsymbol{R}_{i}}{\partial \Delta \boldsymbol{d}}$ denotes the derivative of the residual to the displacement increment, which results in an effective stiffness matrix $\widehat{\mathbf{K}}_{i}$. By rearranging Equation (2.46), a set of equations is obtained from which the change of the displacement increment can be solved:

$$
\widehat{\mathbf{K}}_{i} \delta \boldsymbol{d}=-\boldsymbol{R}_{i} \quad \text { where } \quad \widehat{\mathbf{K}}_{i}=\frac{\partial \boldsymbol{R}_{i}}{\partial \Delta \boldsymbol{d}}=\mathbf{K}_{i}^{\mathrm{int}}-\mathbf{K}_{i}^{\mathrm{ext}}
$$

Here, $\mathbf{K}_{i}^{\text {int }}$ and $\mathbf{K}_{i}^{\text {ext }}$ are stiffness matrices resulting from the derivative of the internal and external force vectors to the displacement increment. The subscript $i$ indicates that these matrices are recomputed in each iteration.

Iterations continue until a relative norm is lower than a predefined tolerance. This relative norm is determined from the residual at the end of each iteration:

$$
\varepsilon=\frac{\left\|\boldsymbol{R}_{i}\right\|}{\left\|\boldsymbol{F}_{i}^{\text {ext }}\right\|}
$$

The Newton-Raphson algorithm is powerful because it can find an approximate solution for Equation (2.43) in a small number of iterations. When the effective stiffness matrix is derived consistently, convergence is asymptotically quadratic. This means that in each iteration near the solution, the error of the solution reduces with the square of the error of the previous iteration. When the initial guess is further away from the solution, convergence is not quadratic and the result of $\boldsymbol{R}_{i}$ might diverge. A disadvantage of the Newton-Raphson algorithm is that it requires the derivative of $\boldsymbol{R}_{i}$ in each iteration, which can be computationally expensive.

\subsection{Distributed forces}

In numerical analysis of pipe-laying the pipe is discretised. Due to this discretisation loads can be applied only on nodes. In this section it is described how uniformly distributed loads, such as gravity, can be applied on a co-rotational beam element.

The uniformly distributed load is defined by $\boldsymbol{q}$, which is a vector that contains a force per unit length in the three directions of the global coordinate system. Based on virtual work nodal forces and moments are derived such that they are statically and kinematically equivalent to this distributed load [11]. Figure 2.9 shows the 
equivalent nodal forces and moments of a beam element subject to a distributed load. The equivalent nodal forces are:

$$
\overline{\boldsymbol{F}}^{\mathrm{q}}=\frac{1}{2} L_{0} \boldsymbol{q}
$$

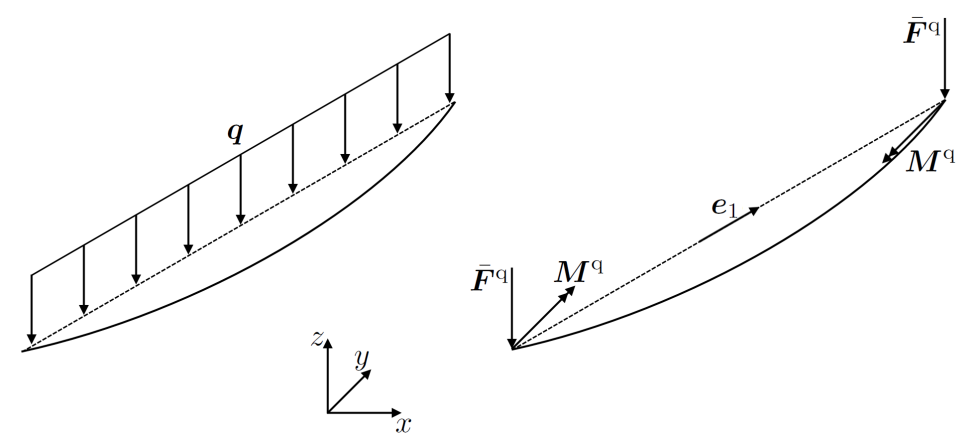

Figure 2.9: Equivalent nodal forces and moments of a beam element subject to a distributed load.

The equivalent nodal moments can also be derived based on virtual work. The moment around the local $x_{\mathrm{L}}$-axis is zero since distributed loads do not contribute to torsional moments. This is exact for an undeformed element and an approximation for a deformed element. Only the component of the distributed load $\boldsymbol{q}$ perpendicular to the $x_{\mathrm{L}}$-axis is used in the calculation of the equivalent moments in the global coordinate system:

$$
\boldsymbol{M}^{\mathrm{q}}=\frac{1}{12}\left(\mathbf{I}-\boldsymbol{e}_{1} \boldsymbol{e}_{1}^{\mathrm{T}}\right) \boldsymbol{q} L_{0}^{2}
$$

The moments at the second node are minus the moments at the first node, such that the equivalent force vector due to distributed forces for a single element becomes:

$$
\boldsymbol{F}^{\mathrm{q}}=\left[\begin{array}{c}
\overline{\boldsymbol{F}}^{\mathrm{q}} \\
\boldsymbol{M}^{\mathrm{q}} \\
\overline{\boldsymbol{F}}^{\mathrm{q}} \\
-\boldsymbol{M}^{\mathrm{q}}
\end{array}\right]
$$

Distributed loads are part of the external force vector. External forces, that are a function of the displacements of the pipe, contribute to the stiffness matrix that is required for good convergence of the Newton-Raphson algorithm, as shown in Section 2.3. This matrix is derived by taking the variation of $\boldsymbol{F}^{\mathrm{q}}$ with respect to the global degrees of freedom. Since $\delta \boldsymbol{q}=\mathbf{0}$, the variation of the forces at each node is zero. The variation of $\boldsymbol{M}^{\mathrm{q}}$ is calculated by:

$$
\delta \boldsymbol{M}^{\mathrm{q}}=\mathbf{K}_{\mathrm{M}} \delta \boldsymbol{p}
$$


with

$$
\mathbf{K}_{\mathrm{M}}=\frac{1}{12} L_{0}^{2}\left[\begin{array}{llll}
\mathbf{0}^{3 \times 3} & \boldsymbol{e}_{1} \boldsymbol{q}^{\mathrm{T}} \mathbf{A}+\boldsymbol{q}^{\mathrm{T}} \boldsymbol{e}_{1} \mathbf{A} & \mathbf{0}^{3 \times 3} & -\boldsymbol{e}_{1} \boldsymbol{q}^{\mathrm{T}} \mathbf{A}-\boldsymbol{q}^{\mathrm{T}} \boldsymbol{e}_{1} \mathbf{A}
\end{array}\right]
$$

This yields the following contribution to the stiffness matrix:

$$
\mathbf{K}^{\mathrm{q}}=\left[\begin{array}{c}
\mathbf{0}^{3 \times 12} \\
\mathbf{K}_{\mathrm{M}} \\
\mathbf{0}^{3 \times 12} \\
-\mathbf{K}_{\mathrm{M}}
\end{array}\right]
$$

\subsection{Second-order axial deformation terms}

In Equation (2.3) the deformed length of an element was approximated by the distance between the nodes. When the beam is curved, the real length is greater than this distance. The correct length can be taken into account by second-order terms for the axial strain, which is shown in Section 2.5.1.

Because of the second-order terms for the axial strain, the relation between the forces and degrees of freedom in the local coordinate system are nonlinear. These forces are derived from strain energy in Section 2.5.2.

By the addition of the second-order terms the accuracy increases, but at the cost of increased computation time. In Section 2.5.3 the necessity of adding these second-order terms is investigated.

\subsubsection{Derivation axial strain}

In the equation for axial strain, additional strain due to curvature is taken into account by $\lambda$ :

$$
\varepsilon_{\mathrm{x}}=\frac{L-L_{0}}{L_{0}}+\lambda
$$

The additional strain is determined from second-order terms of the axial strain [14]:

$$
\varepsilon_{\mathrm{x}}(\xi)=\frac{\partial u_{\mathrm{L}}}{\partial x_{\mathrm{L}}}+\frac{1}{2}\left(\frac{\partial u_{\mathrm{L}}}{\partial x_{\mathrm{L}}}\right)^{2}+\frac{1}{2}\left(\frac{\partial v_{\mathrm{L}}}{\partial x_{\mathrm{L}}}\right)^{2}+\frac{1}{2}\left(\frac{\partial w_{\mathrm{L}}}{\partial x_{\mathrm{L}}}\right)^{2}
$$

The first term of this equation leads to the first term of Equation (2.55). The second term of Equation (2.56) is a second-order term due to axial elongation, which is very small in comparison with the other second-order terms due to the high axial stiffness. The third and fourth terms of Equation (2.56) are responsible for the additional axial strain when the beam is not straight. Equation (2.56) is a function of element coordinate $\xi$, which is 0 at the first node and 1 at the second 
node. The total contribution of the additional axial strain is found by integrating over the element:

$$
\lambda=\int_{0}^{1} \frac{1}{2}\left(\frac{\partial v_{\mathrm{L}}}{\partial x_{\mathrm{L}}}\right)^{2}+\frac{1}{2}\left(\frac{\partial w_{\mathrm{L}}}{\partial x_{\mathrm{L}}}\right)^{2} d \xi
$$

$v_{\mathrm{L}}$ and $w_{\mathrm{L}}$ are the displacements in local $y_{\mathrm{L}}$ and $z_{\mathrm{L}}$-directions within an element and can be determined using the interpolation functions defined in Equation (2.29):

$$
v_{\mathrm{L}}=\boldsymbol{N}_{\mathrm{v}} \boldsymbol{p}_{\mathrm{L}} \quad w_{\mathrm{L}}=\boldsymbol{N}_{\mathrm{w}} \boldsymbol{p}_{\mathrm{L}}
$$

The integral in Equation (2.57) can be solved analytically:

$$
\lambda=\frac{1}{30}\left(2 \phi_{1}^{2}-\phi_{1} \phi_{2}+2 \phi_{2}^{2}\right)+\frac{1}{30}\left(2 \theta_{1}^{2}-\theta_{1} \theta_{2}+2 \theta_{2}^{2}\right)
$$

Alternatively, Equation (2.57) can be determined from the geometry of the deformed beam. An infinitesimal increment of the deformed length of an element can be determined as:

$$
d s^{2}=d x^{2}+d y^{2}+d z^{2} \quad \rightarrow \quad d s=d x \sqrt{1+\left(\frac{d y}{d x}\right)^{2}+\left(\frac{d z}{d x}\right)^{2}}
$$

This can be approximated by a Taylor series expansion: $\sqrt{1+a} \approx 1+a / 2$. The additional strain can be determined by an integrating over the length of the element:

$$
\lambda L_{0}=\int_{0}^{L_{0}}(d s-d x)
$$

The result of this integral is identical to Equation (2.57).

\subsubsection{Element forces and moments}

For elastic material behaviour, forces and moments in the local coordinate system can be determined from strain energy. The strain energy consists of three parts, strain energy due to axial strain, due to bending and due to torsion:

$$
U=U^{\text {axial }}+U^{\text {bending }}+U^{\text {torsion }}
$$

Strain energy due to axial strain is determined by an integral over the length of an element [11]:

$$
U^{\text {axial }}=\frac{1}{2} \int_{0}^{L_{0}} E A \varepsilon_{\mathrm{x}}^{2} d x_{\mathrm{L}}=\frac{1}{2} \frac{E A}{L_{0}}\left(L-L_{0}+\lambda L_{0}\right)^{2}
$$

Strain energy due to bending and that due to torsion are determined similarly:

$$
U^{\text {bending }}=\frac{1}{2} \int_{0}^{L_{0}} E I_{\mathrm{y}} \kappa_{\mathrm{y}}^{2}+E I_{\mathrm{z}} \kappa_{\mathrm{z}}^{2} d x_{\mathrm{L}}
$$


Here, the curvatures $\kappa_{\mathrm{y}}$ and $\kappa_{\mathrm{z}}$ are determined from the second derivative of the displacements $w_{\mathrm{L}}$ and $v_{\mathrm{L}}$ respectively:

$$
\kappa_{\mathrm{y}}=\frac{\partial^{2} w_{\mathrm{L}}}{\partial x_{\mathrm{L}}^{2}} \quad \kappa_{\mathrm{z}}=\frac{\partial^{2} v_{\mathrm{L}}}{\partial x_{\mathrm{L}}^{2}}
$$

These curvatures are second derivatives of Equation (2.58), which are obtained by the second derivatives of the interpolation functions in Equation (2.29) and result in:

$$
U^{\text {bending }}=\frac{1}{2} \frac{E I_{\mathrm{z}}}{L_{0}}\left(2 \theta_{1}^{2}+2 \theta_{1} \theta_{2}+2 \theta_{2}^{2}\right)+\frac{1}{2} \frac{E I_{\mathrm{y}}}{L_{0}}\left(2 \phi_{1}^{2}+2 \phi_{1} \phi_{2}+2 \phi_{2}^{2}\right)
$$

The strain energy due to torsion is determined from the shear strain $\gamma=\left(\psi_{2}-\right.$ $\left.\psi_{1}\right) / L_{0}$ :

$$
U^{\text {torsion }}=\frac{1}{2} \int_{0}^{L_{0}} G J \gamma^{2} d x_{\mathrm{L}}=\frac{1}{2} \frac{G J}{L_{0}}\left(\psi_{2}-\psi_{1}\right)^{2}
$$

The local element forces and moments, which are shown in Figure 2.5, are derived from strain energy using Castigliano's first theorem [27]. Each force and moment is determined by the derivative of the strain energy to the corresponding degree of freedom. The strain energy $U^{\text {axial }}$ is a function of the displacements in axial direction and, due to the second-order terms, of the rotations about the local $y_{\mathrm{L}}$ and $z_{\mathrm{L}}$-axis. Strain energy $U^{\text {bending }}$ is only a function of the rotations about the local $y_{\mathrm{L}}$ and $z_{\mathrm{L}}$-axis, and strain energy $U^{\text {torsion }}$ is only a function of the rotations about the local $x_{\mathrm{L}}$-axis. Therefore, including the correct length of a curved element results in additional terms in the axial forces and the moments about the local $y_{\mathrm{L}}$ and $z_{\mathrm{L}}$-axis.

The axial force and torsion at both nodes are determined as:

$$
\begin{array}{rlrl}
N_{1} & =\frac{\partial U}{\partial u_{1}}=-\frac{E A}{L_{0}}\left(L-L_{0}+\lambda L_{0}\right) & N_{2} & =\frac{\partial U}{\partial u_{2}}=\frac{E A}{L_{0}}\left(L-L_{0}+\lambda L_{0}\right) \\
T_{1}=\frac{\partial U}{\partial \psi_{1}}=-\frac{G J}{L_{0}}\left(\psi_{2}-\psi_{1}\right) & T_{2}=\frac{\partial U}{\partial \phi_{2}}=\frac{G J}{L_{0}}\left(\psi_{2}-\psi_{1}\right)
\end{array}
$$

The bending moments at both nodes are determined as:

$$
\begin{aligned}
M_{\mathrm{y} 1} & =\frac{\partial U}{\partial \theta_{1}}=\frac{E I}{L_{0}}\left(4 \theta_{1}+2 \theta_{2}\right)+N \frac{L_{0}}{30}\left(4 \theta_{1}-\theta_{2}\right) \\
M_{\mathrm{z} 1} & =\frac{E I}{L_{0}}\left(4 \phi_{1}+2 \phi_{2}\right)+N \frac{L_{0}}{30}\left(4 \phi_{1}-\phi_{2}\right) \\
M_{\mathrm{y} 2} & =\frac{E I}{L_{0}}\left(2 \theta_{1}+4 \theta_{2}\right)+N \frac{L_{0}}{30}\left(4 \theta_{2}-\theta_{1}\right) \\
M_{\mathrm{z} 2} & =\frac{E I}{L_{0}}\left(2 \phi_{1}+4 \phi_{2}\right)+N \frac{L_{0}}{30}\left(4 \phi_{2}-\phi_{1}\right)
\end{aligned}
$$


The forces in $y_{\mathrm{L}^{-}}$and $z_{\mathrm{L}}$-direction are determined from the balance of moments and the balance of forces within an element in the local coordinate system:

$$
\begin{aligned}
& F_{\mathrm{y} 1}=\frac{M_{\mathrm{z} 1}+M_{\mathrm{z} 2}}{L_{0}} \\
& F_{\mathrm{z} 1}=-\frac{M_{\mathrm{y} 1}+M_{\mathrm{y} 2}}{L_{0}} \\
& F_{\mathrm{y} 2}=-F_{\mathrm{y} 1} \\
& F_{\mathrm{z} 2}=-F_{\mathrm{z} 1}
\end{aligned}
$$

In Equation (2.22) the forces in the local coordinate system are calculated from a linear relation with the local degrees of freedom. When the second-order terms in the axial strain are taken into account, this relation is nonlinear: $\boldsymbol{F}_{\mathrm{L}}\left(\boldsymbol{p}_{\mathrm{L}}\right)$. The Newton-Raphson algorithm, which is presented in Section 2.3, requires a stiffness matrix. This matrix is derived from the variation of the forces in the local coordinate system. Part of the resulting matrix is constant and identical to that in Equation (2.31), which is renamed $\mathbf{K}_{\mathrm{L}}^{\mathrm{a}}$. The second part, named $\mathbf{K}_{\mathrm{L}}^{\mathrm{b}}$ results from the second-order terms and is not constant.

$$
\mathbf{K}_{\mathrm{L}}=\mathbf{K}_{\mathrm{L}}^{\mathrm{a}}+\mathbf{K}_{\mathrm{L}}^{\mathrm{b}}
$$

The first, constant part of this stiffness matrix, $\mathbf{K}_{\mathrm{L}}^{\mathrm{a}}$, is determined from Equation (2.31):

$$
\mathbf{K}_{\mathrm{L}}^{\mathrm{a}}=\frac{1}{L_{0}}\left[\begin{array}{ccccccc}
0 & 0 & 0 & -E A & 0 & 0 & 0 \\
0 & 0 & 6 \frac{E I}{L_{0}} & 0 & 0 & 0 & 6 \frac{E I}{L_{0}} \\
0 & -6 \frac{E I}{L_{0}} & 0 & 0 & 0 & -6 \frac{E I}{L_{0}} & 0 \\
G J & 0 & 0 & 0 & -G J & 0 & 0 \\
0 & 4 E I & 0 & 0 & 0 & 2 E I & 0 \\
0 & 0 & 4 E I & 0 & 0 & 0 & 2 E I \\
0 & 0 & 0 & E A & 0 & 0 & 0 \\
0 & 0 & -6 \frac{E I}{L_{0}} & 0 & 0 & 0 & -6 \frac{E I}{L_{0}} \\
0 & 6 \frac{E I}{L_{0}} & 0 & 0 & 0 & 6 \frac{E I}{L_{0}} & 0 \\
-G J & 0 & 0 & 0 & G J & 0 & 0 \\
0 & 2 E I & 0 & 0 & 0 & 4 E I & 0 \\
0 & 0 & 2 E I & 0 & 0 & 0 & 4 E I
\end{array}\right]
$$

The second part, $\mathbf{K}_{\mathrm{L}}^{\mathrm{b}}$, is determined from the variation of the second-order terms in Equations (2.68), (2.69) and (2.70). The resulting material stiffness matrix is dependent on the local degrees of freedom. 


$$
\mathbf{K}_{\mathrm{L}}^{\mathrm{b}}=\left[\begin{array}{ccccccc}
0 & -\frac{E A}{30} \Theta_{1} & -\frac{E A}{30} \Phi_{1} & 0 & 0 & -\frac{E A}{30} \Theta_{2} & -\frac{E A}{30} \Phi_{2} \\
0 & \frac{K_{6,2}+K_{12,2}}{L_{0}} & \frac{K_{6,3}+K_{12,3}}{L_{0}} & 0 & 0 & \frac{K_{6,6}+K_{12,6}}{L_{0}} & \frac{K_{6,7}+K_{12,7}}{L_{0}} \\
0 & -\frac{K_{5,2}+K_{11,2}}{L_{0}} & -\frac{K_{5,3}+K_{11,3}}{L_{0}} & 0 & 0 & -\frac{K_{5,6}+K_{11,6}}{L_{0}} & -\frac{K_{5,7}+K_{11,7}}{L_{0}} \\
0 & 0 & 0 & 0 & 0 & 0 & 0 \\
0 & a \Theta_{1}^{2}+\frac{4 L_{0} N}{30} & a \Theta_{1} \Phi_{2} & \frac{E A}{30} \Theta_{1} & 0 & a \Theta_{2} \Theta_{2}-\frac{L_{0} N}{30} & a \Theta_{1} \Phi_{2} \\
0 & a \Theta_{1} \Phi_{1} & a \Phi_{1}^{2}+\frac{4 L_{0} N}{30} & \frac{E A}{30} \Phi_{1} & 0 & a \Phi_{1} \Theta_{2} & a \Phi_{1} \Phi_{2}-\frac{L_{0} N}{30} \\
0 & \frac{E A}{30} \Theta_{1} & \frac{E A}{30} \Phi_{1} & 0 & 0 & \frac{E A}{30} \Theta_{2} & \frac{E A}{30} \Phi_{2} \\
0 & -\frac{K_{6,2}+K_{12,2}}{L_{0}} & -\frac{K_{6,3}+K_{12,3}}{L_{0}} & 0 & 0 & -\frac{K_{6,6}+K_{12,6}}{L_{0}} & -\frac{K_{6,7}+K_{12,7}}{L_{0}} \\
0 & \frac{K_{5,2}+K_{11,2}}{L_{0}} & \frac{K_{5,3}+K_{11,3}}{L_{0}} & 0 & 0 & \frac{K_{5,6}+K_{11,6}}{L_{0}} & \frac{K_{5,7}+K_{11,7}}{L_{0}} \\
0 & 0 & 0 & 0 & 0 & 0 & 0 \\
0 & a \Theta_{1} \Theta_{2}-\frac{L_{0} N}{30} & a \Theta_{2} \Phi_{1} & \frac{E A}{30} \Theta_{2} & 0 & a \Theta_{2}^{2}+\frac{4 L_{0} N}{30} & a \Theta_{2} \Phi_{2} \\
0 & a \Theta_{1} \Phi_{2} & a \Phi_{1} \Phi_{2}-\frac{L_{0} N}{30} & \frac{E A}{30} \Phi_{2} & 0 & a \Phi_{2} \Theta_{2} & a \Phi_{2}^{2}+\frac{4 L_{0} N}{30}
\end{array}\right]
$$

Here, $a$ is a constant and $\Theta_{i}$ and $\Phi_{i}$ are functions of the rotations about the local $y_{\mathrm{L}}$ and $z_{\mathrm{L}}$-axis:

$$
a=E A \frac{L_{0}}{900} \quad \begin{array}{ll}
\Theta_{1}=\left(4 \theta_{1}-\theta_{2}\right) & \Theta_{2}=\left(4 \theta_{2}-\theta_{1}\right) \\
\Phi_{1}=\left(4 \phi_{1}-\phi_{2}\right) & \Phi_{2}=\left(4 \phi_{2}-\phi_{1}\right)
\end{array}
$$

The forces in Equation (2.70) are computed from local moments, thus the contribution of these forces to the stiffness matrix can also be determined from the contribution of these moments. In Equation (2.73), this is represented using $K_{j, k}$, which is an element of matrix $\mathbf{K}_{\mathrm{L}}^{\mathrm{b}}$ in itself.

In the linear relation of Equation (2.22), the computation of the local forces is inexpensive. Additionally, the local stiffness matrix is constant and computed only once. When the curved length of an element is taken into account, this relation becomes nonlinear and a part of the stiffness matrix, $\mathbf{K}_{\mathrm{L}}^{\mathrm{b}}$, is not constant. Thus computational costs per element are increased and the accuracy of the obtained results also increases.

\subsubsection{Necessity of second-order terms}

In Equation (2.69) it can be seen that the nodal moments are defined by two terms. The first term contains the bending stiffness multiplied by a representation of the pipe's curvature. The second term, which results from the second-order terms in the axial strain, can be seen as an increase in bending stiffness due to an axial load. For long slender pipes the bending stiffness is low in comparison with the axial stiffness. When a significant axial load is applied, the nodal moments are dominated by the second term.

The importance of the additional term in the nodal moments is illustrated by an example of a slender pipe subject to an axial load of $F_{\mathrm{x}}=20 \mathrm{kN}$ and a distributed load of $q_{\mathrm{z}}=10 \mathrm{~N} / \mathrm{m}$, as can be seen in Figure 2.10. Properties of the pipe can be seen in Table 2.5 . 


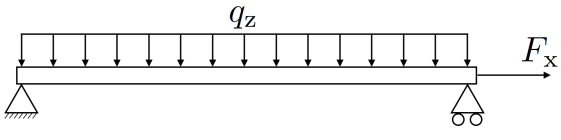

Figure 2.10: Pipe supported at both ends.
Table 2.5: Pipe properties.

\begin{tabular}{lll}
\hline Length & $L$ & $200 \mathrm{~m}$ \\
Diameter & $D$ & $20 \mathrm{~mm}$ \\
Wall Thickness & $t$ & $5 \mathrm{~mm}$ \\
Young's modulus & $E$ & $207 \mathrm{GPa}$ \\
Poisson's ratio & $\nu$ & 0.3
\end{tabular}

This example is computed with and without the second-order terms in the axial strain, the results of which are shown in Figure 2.11. The red line shows the interpolation between the two nodes of an element using Equations (2.29), the blue line is composed of straight lines between the nodes.

In Figure 2.11a it can be seen that the rotations at the nodes are incorrectly large, although the nodal positions are almost correct. This is caused by the equivalent nodal moments, which result from the distributed load, in combination with the low bending stiffness.

Figure 2.11b shows the shape of the deformed pipe when including the secondorder terms in the axial strain. Due to the second-order terms in the axial strain, the bending stiffness of the pipe increases when an axial load is applied. Due to the increased bending stiffness, the rotations at the nodes are computed correctly.

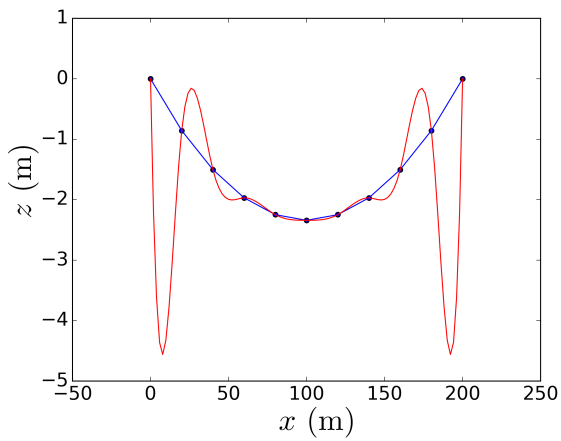

(a) Deformed pipe without second-order terms.

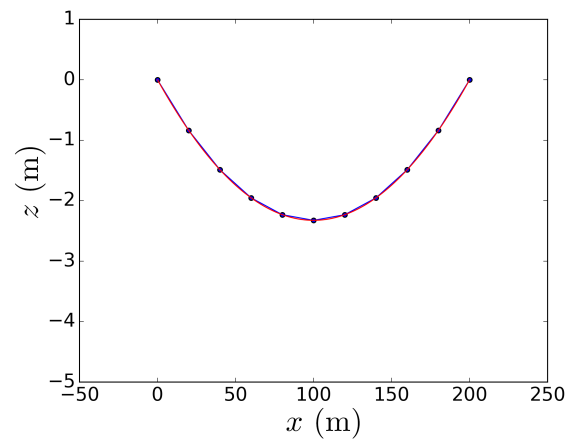

(b) Deformed pipe including second-order terms.

Figure 2.11: Example illustrating the necessity of applying the second-order terms in element forces.

The incorrect large rotations shown in Figure 2.11a can also be a problem in pipelay simulations, where distributed loads are applied to a long slender pipe that is subject to an axial load. In Section 5.4.2 this will be demonstrated on a J-lay simulation. 


\subsection{Closure}

The co-rotational beam element formulation of Crisfield is improved by changing the matrix that rotates the local element forces to the global coordinate system. Using the new rotation matrix a much simpler geometric stiffness matrix is derived.

The new rotation matrix consists of four sub-matrices. The forces are rotated with an element frame, while moments are rotated with nodal frames. Choosing to rotate the moments with the nodal frames is an approximation because the moments are still determined in the local coordinate system that is defined by the element frame. Therefore, the element formulation of Battini-Pacoste, which uses a two-step rotation to overcome this problem, is more accurate when local rotations are large.

The new element formulation is compared with the original formulation of Crisfield and with the formulation of Battini-Pacoste. Results of a two-dimensional example are similar for all three considered formulations. In a three-dimensional example involving torsional moments, the formulation of Crisfield gives incorrect results, of which the error increases when the loads increase. The new formulation and that of Battini-Pacoste give correct results. Both formulations are able to simulate large torsional moments.

The element formulation of Crisfield has higher computational costs than the new formulation and the formulation of Battini-Pacoste. The new element formulation is based on that of Crisfield; it requires the same computations, except for the geometric stiffness matrix. The geometric stiffness matrix of the new formulation is calculated at considerably lower computational costs than that of Crisfield's formulation. The new formulation is also computationally less expensive than the formulation of Battini-Pacoste. Because the new formulation has similar or more accurate results and lower computational cost than the other two formulations, it can be concluded that it is the most efficient of the three.

In practice most models with co-rotational elements will have small local rotations and can benefit from the efficiency of the new element formulation. When large local rotations are involved, the element of Battini-Pacoste is the more accurate.

Due to the nonlinear relation between the internal element forces and the displacements, an iterative method is required to obtain static equilibrium. Here, the Newton-Raphson algorithm is selected as iterative method. When a consistent tangent stiffness matrix is used, the Newton-Raphson algorithm shows asymptotic quadratic convergence.

The pipe is subject to distributed loads such as gravity. Because the co-rotational element formulation only allows nodal loads, equivalent nodal forces and moments were derived. These forces and moments are determined in the global coordinate system.

In a pipe-lay simulation a long slender pipe is subject to a distributed load and an axial force. Due to the axial force, the bending stiffness of the pipe increases. This 
effect can be accounted for by deriving the internal element moment including second-order terms of the axial strain. When the increase of bending stiffness is not accounted for, the equivalent nodal moments in combination with the low bending stiffness can result in an incorrect prediction of nodal rotations. 


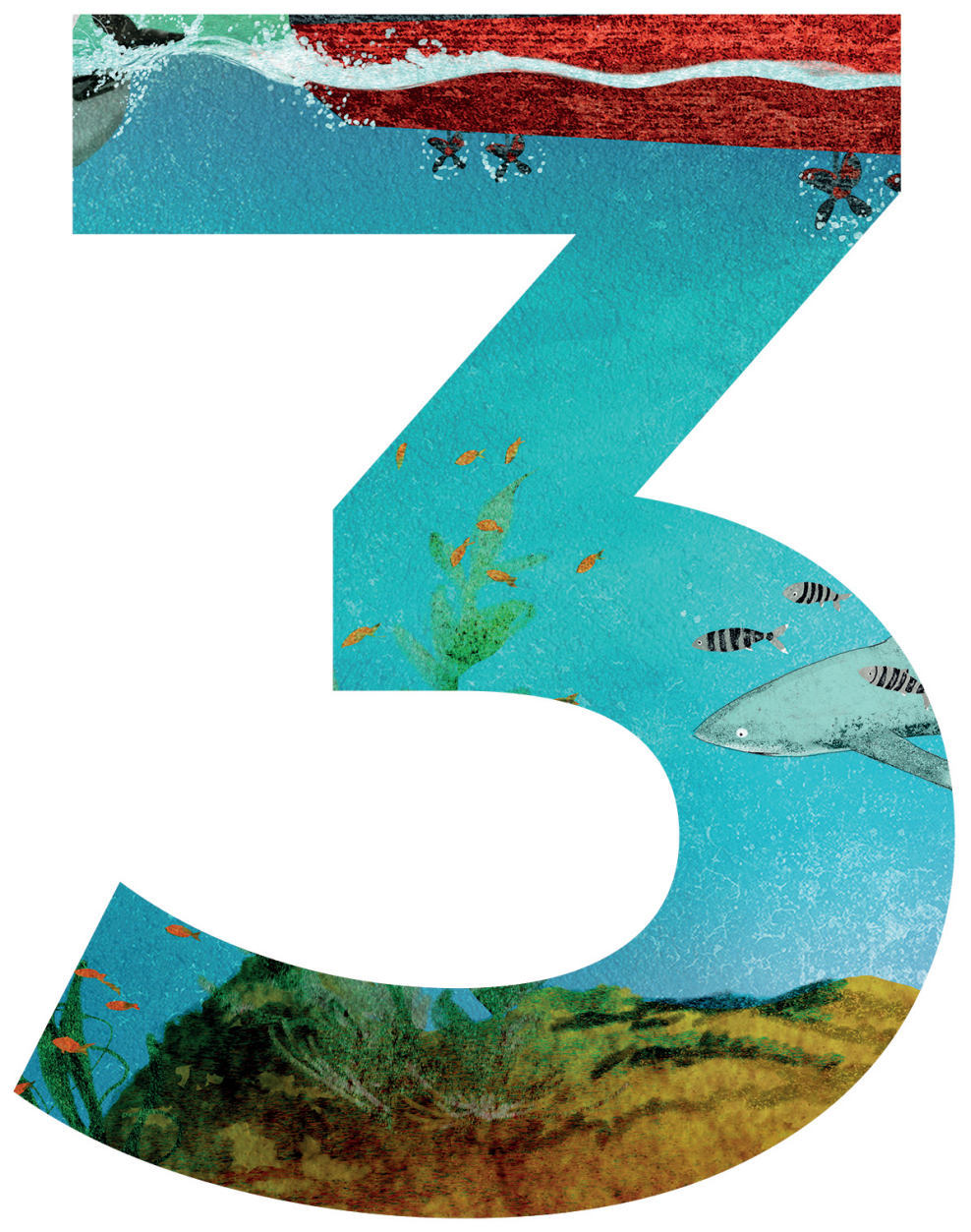




\section{3}

\section{Numerical time integration}

In the dynamic simulation of pipe-laying a mathematical model predicts the behaviour of a pipe subject to applied loads. This mathematical model is an extension of that used for static simulations, which was presented in Chapter 2. The main difference is that the mathematical model for dynamic simulations includes inertia of the pipe and has additional dynamic loads.

Internal forces are included in both the static and the dynamic model. These forces consist of internal element forces, which are described in Section 2.3, and contact forces, which are described in Chapter 4. These internal forces are a function of the displacement of the pipe. A mathematical model for dynamic simulations can also include structural damping in the internal forces, which makes the internal forces a function of the velocities of the pipe. In the pipe-lay model structural damping is disregarded because it is insignificant compared with hydrodynamic damping. Therefore, the internal forces are only a function of the displacements of the pipe and are determined as in the static model.

In the static model external forces consist of gravity loads and buoyancy loads, which are functions of the displacements of the pipe. In the dynamic model external forces can additionally include terms such as hydrodynamic damping, inertia of the water and wave loads. Models for these static and dynamic loads are considered in Chapter 5. Due to these damping and inertia terms the external forces can also be a function of the velocity and acceleration of the pipe.

The dynamic model describes motions of a structure subject to a dynamic loading by a nonlinear equation. This equation of motion is well known, see for example $[11,59]$ :

$$
\mathbf{M} \ddot{\boldsymbol{d}}+\boldsymbol{F}^{\mathrm{int}}=\boldsymbol{F}^{\mathrm{ext}}
$$

Here, $\mathbf{M} \ddot{\boldsymbol{d}}$ represents the inertia of the structure, $\boldsymbol{F}^{\text {int }}$ is a vector with the internal forces and $\boldsymbol{F}^{\text {ext }}$ is a vector with the external forces. 
The equation of motion is continuous in time and is approximated by a numerical integration method, which evaluates the mathematical model at discrete points in a time interval. These discrete points are separated by a time step $\Delta t$ and are evaluated in chronological order. In this work the state of the structure is known at current time $t$ and is unknown at the end of the time increment $t+\Delta t$. In Section 3.1 the time integration method, that will be used in the efficient pipe-lay model, is selected from literature on the basis of a set of requirements.

In the equation of motion the inertia term is described by a mass matrix $\mathbf{M}$ and accelerations of the pipe $\ddot{\boldsymbol{d}}$. In general the mass matrix is a function of the displacements of the pipe. This matrix can be simplified to a diagonal matrix that is independent of the pipe's displacements. In Section 3.2 it is investigated whether results remain sufficiently accurate when this reduced matrix is used.

A structure has as many natural frequencies as it has degrees of freedom, including high frequencies. High natural frequencies are usually physically not relevant, because they are not excited and are quickly damped. In a dynamic simulation time steps can be larger than the period of high frequencies that are not of interest. Because the time steps are larger than the period of these high frequencies, the simulation cannot predict the behaviour of the structure accurately. These high frequencies can be excited at the start of a dynamic simulation, when a structure is suddenly loaded. In Section 3.3 it will be shown that these high frequencies at the start of a simulation can be damped by an appropriate initialisation procedure.

\subsection{Method selection}

To select the time integration method best suited for efficient analysis of pipelaying, a set of requirements is composed. These requirements are based on the order of accuracy, stability and efficiency.

The order of accuracy of a method indicates the rate at which the discretisation error decreases when the time step is decreased. In a higher-order method this error decreases faster than in a lower-order method. A method is stable when the numerical error does not grow with time. In unstable methods the error can grow with time, resulting in an incorrect solution. The efficiency of a method is determined by the computational costs at equal accuracy.

The requirements for the time integration method are:

- The method needs to be second-order accurate: In structural dynamics second-order accurate methods are generally superior to first-order accurate methods $[28,60]$ because their higher order of accuracy comes with minimal increase in computational costs.

- The method needs to be stable when time steps are larger than the period of high frequencies: In the numerical model the pipe has high natural 
frequencies which are physically not relevant. In conditionally stable methods the maximum time step is determined in proportion to the period of the highest frequency in the structure. Evaluating the model with such small time steps would be highly inefficient. Unconditionally stable algorithms, where the time step is not restricted by the highest frequency, are therefore preferred. Formally, these algorithms are unconditionally stable only for linear systems. They can be applied in nonlinear systems as well, when possible instabilities are suppressed by damping.

- The method needs to have controllable numerical damping: The physically irrelevant high frequencies of the pipe can be exited during the dynamic simulation. When this happens, it is desirable that the algorithm can damp these frequencies. The numerical damping should not affect the low frequencies substantially.

- The method needs to be efficient: The goal of this research is to create an efficient pipe-lay model. An efficient time integration method is paramount to achieving this goal. When more than one method meets the previous three requirements, the method with the lowest computational costs is selected.

Many methods for time integration exist today [59], which can be split into two groups: explicit methods [21] and implicit methods [60]. Explicit methods calculate a solution to the equation of motion at the end of a time increment directly from the solution at the current time. Implicit methods find a solution at the end of a time increment by solving an equation involving both the solution at the current time and the unknowns at the end of the time increment.

In contrast to implicit methods, explicit methods do not require a system of equations to be solved when a diagonal mass matrix is used. Therefore, computational costs per time increment of explicit methods are lower than to those of implicit methods. In the pipe-lay model the pipe is modelled with a string of beam elements. The stiffness matrix becomes a band-matrix with a narrow band and solving the system of equations is computationally inexpensive, which limits the difference in computational costs per time step between implicit and explicit methods.

Stability of explicit methods is dependent on the size of the time step; the maximum time step is determined in proportion to the period of the highest frequency in the structure. Due to the high natural frequencies of the pipe, time steps must be very small. In an implicit method the time step does not have this restriction. Because the time integration method needs to evaluate the equation of motion over a long time period, implicit methods have lower computational costs over the entire time interval. Furthermore, results of implicit methods are generally more accurate than those of explicit methods because implicit methods evaluate the force balance of the equation of motion at the end of the time increment. In conclusion, explicit methods can be discarded because of the stability and efficiency requirements. 
Implicit time integration schemes can be split into three groups: single-step, multistep and sub-step methods. Single-step methods, such as the Newmark family of methods [42] or the Wilson- $\theta$ method [63], determine a solution from the nodal positions both at the current time and at the end of the time increment. Multistep methods, such as the Houbolt [31], determine a solution from also using the nodal positions before the current time. Sub-step methods, such as the Bathe [4] method, determine a solution from the nodal positions at the current time, at one or more intermediate times and at the end of the time increment.

Multi-step methods require a special starting procedure, as the nodal positions at previous time steps are not known. The Houbolt method has no controllable numerical damping. Furthermore, [24] concluded that, compared to the Newmark family of methods, the Houbolt method requires significantly more time steps for comparable accuracy. The Houbolt method does not fulfil the requirements on controllable damping and efficiency.

Sub-step methods require more computation time than single-step methods, because the equation of motion is also evaluated between the current time and the end of the time increment. Thus sub-step methods need a significant increase in accuracy to be more efficient. The Bathe method does not have controllable numerical damping, so it does not fulfil the requirements on controllable damping and efficiency.

Of the single-step methods the Wilson- $\theta$ method is known to overshoot substantially at the start of a simulation when large time steps are used [60], which means it overestimates displacement increments. This is considerably less of a problem for the Newmark family of methods. For comparable accuracy the Newmark family of methods requires significantly fewer time steps than the Wilson- $\theta$ method [24]. The methods from the Newmark family are the remaining candidates for the time integration method in the efficient pipe-lay model.

Three different methods from the Newmark-family are presented in this chapter: the Newmark- $\beta$ method [42], the Hilber-Hughes-Taylor- $\alpha$ (HHT- $\alpha$ ) method [29] and the Generalised- $\alpha$ method [10]. The Newmark- $\beta$ method is second-order accurate but loses this accuracy when numerical damping is included. The HHT- $\alpha$ and Generalised- $\alpha$ methods remain second-order accurate when numerical damping is included. All three methods are unconditionally stable for linear systems and have controllable numerical damping.

The Newmark- $\beta$ method, the HHT- $\alpha$ method and the Generalised- $\alpha$ method are presented in Sections 3.1.1-3.1.3. In Section 3.1.4 their results are compared and the method that best fits the requirements is selected.

\subsubsection{Newmark- $\beta$ method}

The Newmark- $\beta$ method [42] is one of the most frequently used time integration schemes in the numerical analysis of dynamic structures. It has two controllable 
parameters, $\beta$ and $\gamma$, which can be tuned for accuracy and stability. The derivation of this method can be found in many books such as $[15,59]$. This method is also the basis for the HHT- $\alpha$ method and Generalised- $\alpha$ method.

The Newmark- $\beta$ method defines the velocity at the end of a time increment and the displacement increment as functions of its controllable parameters. This definition can be rewritten such that the accelerations and velocities at the end of a time increment are functions of the displacement increment. Then the displacement increment is the only unknown variable. This unknown can be solved from a discretised equation of motion using the Newton-Raphson algorithm.

The nodal velocities $\dot{\boldsymbol{d}}_{t}$ and accelerations $\ddot{\boldsymbol{d}}_{t}$ at the current time are known. The nodal velocities $\dot{\boldsymbol{d}}_{t+\Delta t}$ at the end of a time step and displacement increment $\Delta \boldsymbol{d}$ are calculated by:

$$
\begin{aligned}
& \dot{\boldsymbol{d}}_{t+\Delta t}=\dot{\boldsymbol{d}}_{t}+\Delta t \ddot{\boldsymbol{d}}_{\gamma} \\
& \Delta \boldsymbol{d}=\Delta t \dot{\boldsymbol{d}}_{t}+\frac{1}{2} \Delta t^{2} \ddot{\boldsymbol{d}}_{\beta} \quad \text { where } \quad \begin{array}{l}
\ddot{\boldsymbol{d}}_{\gamma}=(1-\gamma) \ddot{\boldsymbol{d}}_{t}+\gamma \ddot{\boldsymbol{d}}_{t+\Delta t} \\
\ddot{\boldsymbol{d}}_{\beta}=(1-2 \beta) \ddot{\boldsymbol{d}}_{t}+2 \beta \ddot{\boldsymbol{d}}_{t+\Delta t}
\end{array}
\end{aligned}
$$

Equations (3.2) are rewritten such that the velocities and accelerations are expressed as functions of the displacement increment:

$$
\begin{aligned}
\ddot{\boldsymbol{d}}_{t+\Delta t} & =a_{0} \Delta \boldsymbol{d}-a_{2} \dot{\boldsymbol{d}}_{t}-a_{3} \ddot{\boldsymbol{d}}_{t} \\
\dot{\boldsymbol{d}}_{t+\Delta t} & =a_{1} \Delta \boldsymbol{d}-a_{4} \dot{\boldsymbol{d}}_{t}-a_{5} \ddot{\boldsymbol{d}}_{t}
\end{aligned}
$$

Here, $a_{i}$ are constants that are determined by the time step and by the controllable parameters $\beta$ and $\gamma$. These constants have different dimensions.

$$
\begin{array}{lll}
a_{0}=\frac{1}{\beta \Delta t^{2}} & a_{1}=\frac{\gamma}{\beta \Delta t} & a_{2}=\frac{1}{\beta \Delta t} \\
a_{3}=\frac{1}{2 \beta}-1 & a_{4}=\frac{\gamma}{\beta}-1 & a_{5}=\frac{\Delta t}{2}\left(\frac{\gamma}{\beta}-2\right)
\end{array}
$$

The displacement increment is solved from the equation of motion, Equation (3.1), which is discretised in time. Since all variables at the current time are known, the discretised equation of motion is a function of the variables at the end of time increment:

$$
\mathbf{M} \ddot{\boldsymbol{d}}_{t+\Delta t}+\boldsymbol{F}_{t+\Delta t}^{\mathrm{int}}=\boldsymbol{F}_{t+\Delta t}^{\mathrm{ext}}
$$

The velocity and force vectors in this equation have a subscript that indicates the end of the time increment. $\mathbf{M}$ is the mass matrix at the end of the current time increment, the subscript is omitted for readability. This equation is solved using the Newton-Raphson algorithm, as explained in Section 2.3 for static simulations. In this algorithm the displacement increment that makes the residual $\boldsymbol{R}$ equal to a vector of zeros is determined using an effective stiffness matrix $\widehat{\mathbf{K}}$. The residual is found by moving all terms of Equation (3.5) to the left and substituting Equation $(3.3)$ :

$$
\boldsymbol{R}=\boldsymbol{F}_{t+\Delta t}^{\mathrm{int}}-\boldsymbol{F}_{t+\Delta t}^{\mathrm{ext}}+\mathbf{M}\left(a_{0} \Delta \boldsymbol{d}-a_{2} \dot{\boldsymbol{d}}_{t}-a_{3} \ddot{\boldsymbol{d}}_{t}\right)
$$


The effective stiffness matrix is determined as shown in Section 2.3. The internal and external force vectors are linearised, resulting in an internal and external stiffness matrix. Additionally, the derivative of the inertia term with respect to the displacement increment is included in the effective stiffness matrix:

$$
\widehat{\mathbf{K}}=\mathbf{K}^{\text {int }}-\mathbf{K}^{\text {ext }}+a_{0} \mathbf{M}+\mathbf{M}^{\prime}\left(a_{0} \Delta \boldsymbol{d}-a_{2} \dot{\boldsymbol{d}}_{t}-a_{3} \ddot{\boldsymbol{d}}_{t}\right)
$$

Here $\mathbf{M}^{\prime}$ is the derivative of the mass matrix to the displacement increment. In Section 3.2 a consistent mass matrix is derived, which is a function of the displacement increment. It will be shown that this matrix can be simplified to a constant diagonal mass matrix, for which the last term of Equation (3.7) is zero, such that the equivalent stiffness matrix reduces to:

$$
\widehat{\mathbf{K}}=\mathbf{K}^{\text {int }}-\mathbf{K}^{\text {ext }}+a_{0} \mathbf{M}
$$

When Equation (3.8) is used in combination with a non-constant mass matrix, the number of iterations increases because the stiffness matrix is not consistent.

\section{Parameters $\beta$ and $\gamma$}

In the Newmark- $\beta$ method two parameters can be controlled. The parameter $\gamma$ determines numerical damping; when $\gamma=1 / 2$ there is no numerical damping and when $\gamma>1 / 2$ there is numerical damping. The resulting displacements from this method are second order accurate only when there is no numerical damping. With different choices of $\beta$, different methods can be obtained, as can be seen in Table 3.1. The parameters also have an influence on the numerical stability. For linear systems the method is unconditionally stable when [24]:

$$
\gamma \geq \frac{1}{2} \quad \beta \geq \frac{1}{4}\left(\gamma+\frac{1}{2}\right)^{2}
$$

Table 3.1: Choices of parameter $\beta[21]$

\begin{tabular}{lll}
\hline$\beta$ & $\gamma$ & Method \\
\hline $1 / 4$ & $1 / 2$ & Average acceleration / trapezoidal rule \\
$1 / 6$ & $1 / 2$ & Linear acceleration \\
0 & $1 / 2$ & Explicit central difference \\
\hline
\end{tabular}

\subsubsection{Hilber-Hughes-Taylor- $\alpha$ method}

The HHT- $\alpha$ method [29] is based on the Newmark- $\beta$ method, using the same equations to determine the velocities and accelerations. It has one controllable 
parameter, $\alpha$. Newmark- $\beta$ parameters $\beta$ and $\gamma$ are dependent on $\alpha$. This method remains second-order accurate when there is numerical damping. The HHT- $\alpha$ method can suffer from overshoot and high frequencies are damped gradually over time.

In the HHT- $\alpha$ method the equation of motion is discretised as:

$$
\mathbf{M} \ddot{\boldsymbol{d}}_{t+\Delta t}+\boldsymbol{F}_{\alpha}^{\mathrm{int}}=\boldsymbol{F}_{\alpha}^{\mathrm{ext}}
$$

Here, the internal and external forces are determined by scaling the force increments with $(1-\alpha)$ :

$$
\begin{aligned}
& \boldsymbol{F}_{\alpha}^{\text {int }}=\boldsymbol{F}_{t}^{\text {int }}+(1-\alpha) \Delta \boldsymbol{F}^{\text {int }} \\
& \boldsymbol{F}_{\alpha}^{\text {ext }}=\boldsymbol{F}_{t}^{\text {ext }}+(1-\alpha) \Delta \boldsymbol{F}^{\text {ext }}
\end{aligned}
$$

The displacement increment is solved from Equation (3.10) using the NewtonRaphson algorithm, as explained in Section 2.3 for the static simulation. The residual is determined by moving all terms of Equation (3.10) to the left and substituting Equation (3.3):

$$
\boldsymbol{R}=\boldsymbol{F}_{\alpha}^{\mathrm{int}}-\boldsymbol{F}_{\alpha}^{\mathrm{ext}}+\mathbf{M}\left(a_{0} \Delta \boldsymbol{d}-a_{2} \dot{\boldsymbol{d}}_{t}-a_{3} \ddot{\boldsymbol{d}}_{t}\right)
$$

The Newton-Raphson algorithm requires an effective stiffness matrix, which is determined as shown in Section 2.3. When assuming a constant mass matrix, the effective stiffness matrix becomes:

$$
\widehat{\mathbf{K}}=a_{0} \mathbf{M}+(1-\alpha)\left(\mathbf{K}^{\text {int }}-\mathbf{K}^{\text {ext }}\right)
$$

\section{Parameter $\alpha$}

There are three parameters in the HHT- $\alpha$ method: parameter $\alpha$ and Newmark- $\beta$ parameters $\beta$ and $\gamma$. The method is second-order accurate and unconditionally stable for linear systems when [29]:

$$
0 \leq \alpha \leq \frac{1}{3} \quad \beta=\frac{(1+\alpha)^{2}}{4} \quad \gamma=\frac{1}{2}+\alpha
$$

When $\alpha=0$ this method reduces to the Newmark- $\beta$ method with average acceleration. When $\alpha>0$ there is numerical damping added to the system, which damps mainly higher frequencies [29].

\subsubsection{Generalised- $\alpha$ method}

The Generalised- $\alpha$ method [10] is a combination of the HHT- $\alpha$ method and the Wood-Bossak-Zienkiewicz- $\alpha$, or WBZ- $\alpha$ method [64]. The WBZ- $\alpha$ method is a 
modification of the Newmark- $\beta$ method that applies a parameter $\alpha$ on the inertia term in the equation of motion, in a similar way to the HHT- $\alpha$ method that applies a parameter $\alpha$ on the force vectors in the equation of motion. In the Generalised- $\alpha$ method, the parameter $\alpha$ of the HHT- $\alpha$ method is renamed $\alpha_{\mathrm{f}}$ and a parameter $\alpha$ of the WBZ- $\alpha$ method is renamed $\alpha_{\mathrm{m}}$.

The Generalised- $\alpha$ method has better damping properties than the HHT- $\alpha$ method. It introduces a new controllable parameter $\rho_{\infty}$, which is derived to minimise the dissipation of low frequencies while damping the high frequencies.

In the Generalised- $\alpha$ method the discretised equation of motion is defined as:

$$
(\mathbf{M} \ddot{\boldsymbol{d}})_{\alpha_{\mathrm{m}}}+\boldsymbol{F}_{\alpha_{\mathrm{f}}}^{\mathrm{int}}=\boldsymbol{F}_{\alpha_{\mathrm{f}}}^{\mathrm{ext}}
$$

The first term of the discretised equation of motion is defined as:

$$
(\mathbf{M} \ddot{\boldsymbol{d}})_{\alpha_{\mathrm{m}}}=\left(1-\alpha_{\mathrm{m}}\right) \mathbf{M} \ddot{\boldsymbol{d}}_{t+\Delta t}+\alpha_{\mathrm{m}} \mathbf{M}_{t} \ddot{\boldsymbol{d}}_{t}
$$

Equation (3.15) is solved using the Newton-Raphson algorithm, as explained in Section 2.3 for the static simulation. The equivalent force vector is determined by moving all terms of Equation (3.15) to the left and substituting Equation (3.3):

$$
\boldsymbol{R}=\boldsymbol{F}_{\alpha_{\mathrm{f}}}^{\mathrm{int}}-\boldsymbol{F}_{\alpha_{\mathrm{f}}}^{\mathrm{ext}}+\left(1-\alpha_{\mathrm{m}}\right) \mathbf{M}\left(a_{0} \Delta \boldsymbol{d}-a_{2} \dot{\boldsymbol{d}}_{t}-a_{3} \ddot{\boldsymbol{d}}_{t}\right)+\alpha_{\mathrm{m}} \mathbf{M}_{t} \ddot{\boldsymbol{d}}_{t}
$$

The Newton-Raphson algorithm requires an effective stiffness matrix, which is determined as shown in Section 2.3. When assuming a constant mass matrix, the effective stiffness matrix becomes:

$$
\widehat{\mathbf{K}}=\left(1-\alpha_{\mathrm{m}}\right) a_{0} \mathbf{M}+\left(1-\alpha_{\mathrm{f}}\right)\left(\mathbf{K}^{\mathrm{int}}-\mathbf{K}^{\mathrm{ext}}\right)
$$

\section{Parameters $\alpha_{\mathrm{m}}$ and $\alpha_{\mathrm{f}}$}

From equations (3.15) and (3.16) it can be seen that the Generalised- $\alpha$ method reduces to the HHT- $\alpha$ method when $\alpha_{\mathrm{m}}=0$. When $\alpha_{\mathrm{m}}=0$ and $\alpha_{\mathrm{f}}=0$, it reduces to the Newmark- $\beta$ method. The Newmark- $\beta$ parameters are determined as:

$$
\gamma=\frac{1}{2}-\alpha_{\mathrm{m}}+\alpha_{\mathrm{f}} \quad \beta=\frac{1}{4}\left(\frac{1}{2}+\gamma\right)^{2}
$$

This ensures that the Generalised- $\alpha$ method is unconditionally stable for linear systems and is second-order accurate. $\alpha_{\mathrm{m}}$ and $\alpha_{\mathrm{f}}$ are determined from the new parameter $\rho_{\infty}[10]$ :

$$
\alpha_{\mathrm{m}}=\frac{2 \rho_{\infty}-1}{\rho_{\infty}+1} \quad \alpha_{\mathrm{f}}=\frac{\rho_{\infty}}{\rho_{\infty}+1}
$$

When $\rho_{\infty}=1$ there is no damping, when $\rho_{\infty}<1$ there is damping. When $\rho_{\infty}=0$ the high frequency response is damped after one time step. More on these parameters can be found in [59]. 


\subsubsection{Comparison}

The three remaining candidates for the time integration method best suited for efficient analysis of pipe-laying are the Newmark- $\beta$ method, the HHT- $\alpha$ method and the Generalised- $\alpha$ method. They are evaluated on the basis of the requirements presented at the beginning of Section 3.1. The candidates are compared using a dynamic simulation of a cantilevered pipe. At the pipe's tip two equally large moments of $500 \mathrm{kNm}$ are applied, as can be seen in Figure 3.1. The pipe is divided into ten co-rotational beam elements, properties of the pipe are given in Table 3.2. Displacements are calculated over a period of $60 \mathrm{~s}$, using a time step of $0.1 \mathrm{~s}$.

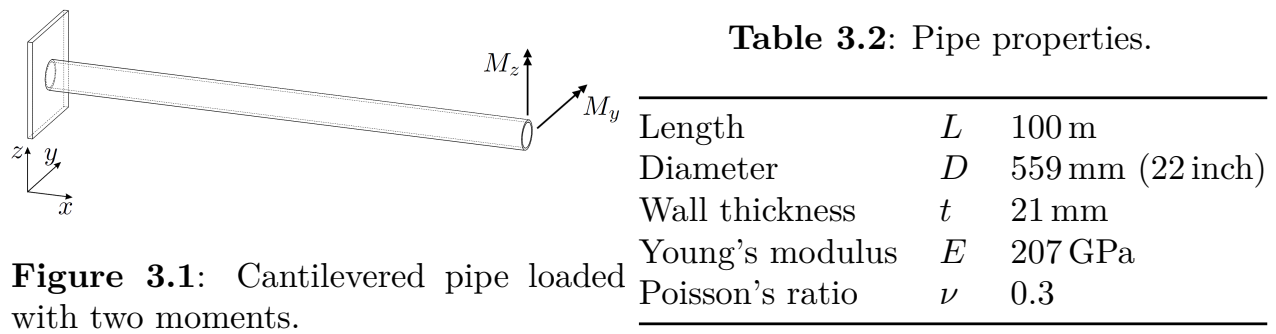

The three candidates are compared by investigating their numerical damping and efficiency. The Newmark- $\beta$ method is applied without numerical damping, such that it is second-order accurate. The other two candidates are second-order accurate independent of their parameters, which are chosen to include numerical damping as can be seen in Table 3.3.

To compare the numerical damping of the three candidates, the total displacement of the tip is determined in the frequency domain. Figure $3.2 \mathrm{a}$ shows the displacement of the pipe's tip in the time domain using the Newmark- $\beta$ method. This solution is transformed to the frequency domain using a fast Fourier transform algorithm [12]. The results of the three candidates are depicted in Figures 3.2b-3.2d.

The numerical damping has little influence on the first two natural frequencies, therefore these figures zoom in on the third and higher natural frequencies. The HHT- $\alpha$ method damps the third natural frequency (around $1 \mathrm{~Hz}$ ) and higher, while the Generalised- $\alpha$ method damps only the fifth natural frequency (around $2.5 \mathrm{~Hz}$ ) and higher. For both these methods, the controllable parameter can be adjusted such that the damping on the higher natural frequencies is sufficient while not affecting the low frequencies too much. The Newmark- $\beta$ method cannot have numerical damping without losing its second-order accuracy.

Table 3.3 shows the required number of iterations and computational costs of the cantilevered pipe example for all three candidates. The Newmark- $\beta$ method requires the most iterations because it has no numerical damping. It still has 


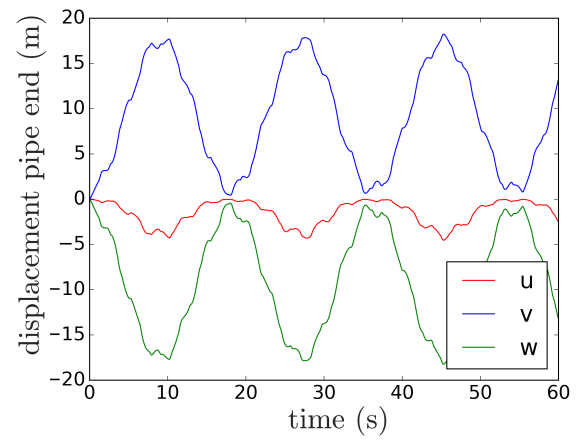

(a) Displacements of pipe tip in global $x$-, $y$ - and $z$-direction.

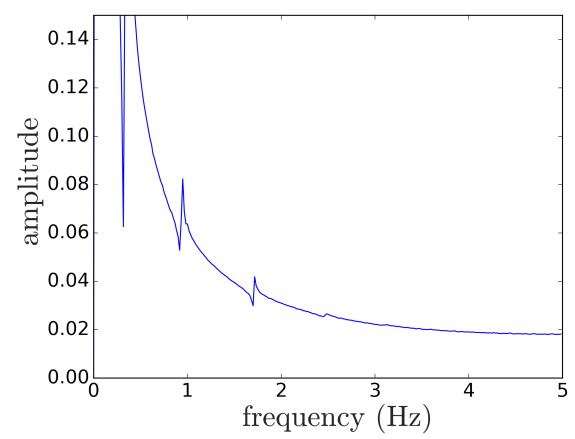

(c) FFT of total pipe tip displacement using the HHT- $\alpha$ method with $\alpha=0.03$.

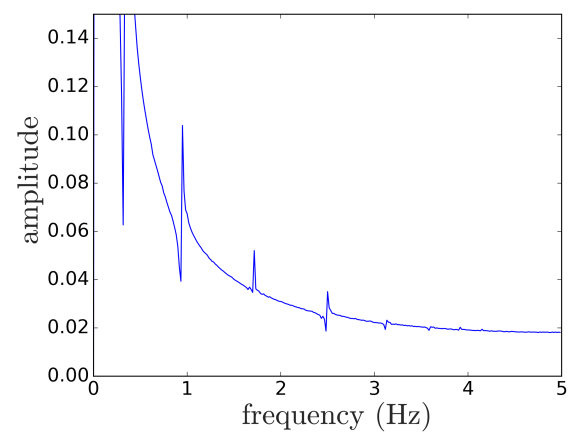

(b) FFT of total pipe tip displacement using the Newmark- $\beta$ method without numerical damping.

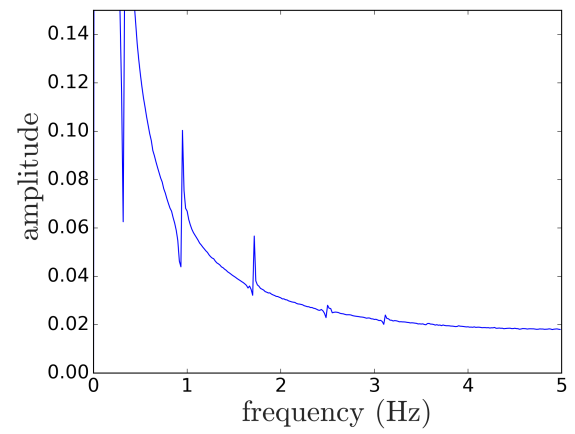

(d) FFT of total pipe tip displacement using the Generalised- $\alpha$ method with $\rho_{\infty}=0.9$.

Figure 3.2: Results of the cantilevered pipe example obtained with the HHT- $\alpha$ method and the Generalised- $\alpha$ method.

low computational costs due to the short computational time per iteration. The Generalised- $\alpha$ method requires fewest iterations but has the highest computational costs. This is a consequence of the extra computations in determining the residual and the effective stiffness matrix. The HHT- $\alpha$ method requires a low number of iterations and has the lowest computational costs.

The method best suited for efficient analysis of pipe-laying is selected by revisiting the requirements set at the beginning of Section 3.1. The Newmark- $\beta$ method is discarded because it cannot have numerical damping while remaining second-order accurate. The HHT- $\alpha$ method and Generalised- $\alpha$ method both are second-order accurate and both have controllable numerical damping. The HHT- $\alpha$ method is more efficient than the Generalised- $\alpha$ method, therefore it is selected as the integration method. At the start of a simulation this method can 
Table 3.3: Comparison of number of iterations and computational costs of the cantilevered pipe simulation using the Newmark- $\beta$ method, the HHT- $\alpha$ method and the Generalised- $\alpha$ method.

\begin{tabular}{llll}
\hline Method & Parameter & Number of iterations & Computational costs \\
\hline Newmark- $\beta$ & $\gamma=1 / 2, \beta=1 / 4$ & 1802 & $0.75 \mathrm{~s}$ \\
HHT- $\alpha$ & $\alpha=0.03$ & 1233 & $0.60 \mathrm{~s}$ \\
Generalised- $\alpha$ & $\rho_{\infty}=0.9$ & 1220 & $0.88 \mathrm{~s}$ \\
\hline
\end{tabular}

suffer from overshoot, which is high-frequency behaviour that is not quickly damped. A procedure for overcoming this issue is investigated in Section 3.3.

\subsection{Mass matrix}

To evaluate the equation of motion, a system mass matrix needs to be computed. This system mass matrix is assembled from element mass matrices. In a corotational frame of reference these element mass matrices are obtained by rotating a local mass matrix to the global coordinate system.

When the local mass matrix $\mathbf{M}_{\mathrm{L}}$ is derived from the same interpolation functions that are used in the derivation of the stiffness matrix, it is called consistent. In each Newton-Raphson iteration a consistent mass matrix is rotated to the global coordinate system by rotation matrix $\mathbf{R}$ :

$$
\mathbf{M}=\mathbf{R}^{\mathrm{T}} \mathbf{M}_{\mathrm{L}} \mathbf{R}
$$

To reduce computational cost it is beneficial to have a constant mass matrix. A constant matrix needs to be computed only once and does not require an additional term in the effective stiffness matrix, as shown in Equation (3.7). A constant mass matrix can be obtained by simplifying the consistent mass matrix to a diagonal matrix that has equal terms in all translational directions and equal terms in all rotational directions. This simplification can have an influence on the accuracy of the results, as investigated in this section.

\subsubsection{Consistent}

The derivation of the consistent mass matrix for a beam element can be found in many textbooks, such as $[11,48]$. In this derivation it is assumed that the mass of the element remains on its neutral line and the length of the element is assumed constant. The mass matrix is determined by an integral over the original length of the element:

$$
\mathbf{M}_{\mathrm{L}}=\rho A \int_{L_{0}} \mathbf{N}^{\mathrm{T}} \mathbf{N} d x \quad \text { with } \quad \mathbf{N}=\left[\begin{array}{lll}
\boldsymbol{N}_{\mathrm{u}} & \boldsymbol{N}_{\mathrm{v}} & \boldsymbol{N}_{\mathrm{w}}
\end{array}\right]^{\mathrm{T}}
$$


Here, $\rho$ is the density and $A$ is the cross-sectional area. $\boldsymbol{N}_{\mathrm{u}}, \boldsymbol{N}_{\mathrm{v}}$ and $\boldsymbol{N}_{\mathrm{w}}$ are vectors with the shape functions, as shown in Equation (2.27). The consistent mass matrix is found by solving the integral:

$$
\mathbf{M}_{\mathrm{L}}=\frac{m}{420}\left[\begin{array}{cccccccccccc}
140 & 0 & 0 & 0 & 0 & 0 & 70 & 0 & 0 & 0 & 0 & 0 \\
0 & 156 & 0 & 0 & 0 & 22 L_{0} & 0 & 54 & 0 & 0 & 0 & -13 L_{0} \\
0 & 0 & 156 & 0 & -22 L_{0} & 0 & 0 & 0 & 54 & 0 & 13 L_{0} & 0 \\
0 & 0 & 0 & 0 & 0 & 0 & 0 & 0 & 0 & 0 & 0 & 0 \\
0 & 0 & -22 L_{0} & 0 & 4 L_{0}^{2} & 0 & 0 & 0 & -13 L_{0} & 0 & -3 L_{0}^{2} & 0 \\
0 & 22 L_{0} & 0 & 0 & 0 & 4 L_{0}^{2} & 0 & 13 L_{0} & 0 & 0 & 0 & -3 L_{0}^{2} \\
70 & 0 & 0 & 0 & 0 & 0 & 140 & 0 & 0 & 0 & 0 & 0 \\
0 & 54 & 0 & 0 & 0 & 13 L_{0} & 0 & 156 & 0 & 0 & 0 & -22 L_{0} \\
0 & 0 & 54 & 0 & -13 L_{0} & 0 & 0 & 0 & 156 & 0 & 22 L_{0} & 0 \\
0 & 0 & 0 & 0 & 0 & 0 & 0 & 0 & 0 & 0 & 0 & 0 \\
0 & 0 & 13 L_{0} & 0 & -3 L_{0}^{2} & 0 & 0 & 0 & 22 L_{0} & 0 & 4 L_{0}^{2} & 0 \\
0 & -13 L_{0} & 0 & 0 & 0 & -3 L_{0}^{2} & 0 & -22 L_{0} & 0 & 0 & 0 & 4 L_{0}^{2}
\end{array}\right]
$$

Here $L_{0}$ is the original length of an element and $m=\rho A L_{0}$ is the mass of an element.

\subsubsection{Lumped}

A lumped mass matrix approximates the inertia of an element. In literature, different methods for creating a lumped mass matrix can be found, all of which result in a diagonal matrix.

In Row-sum lumping [59] the values in each row are summed and the sum is put on the diagonal. In this method, values on the diagonal can become negative, which can be seen when summing the fifth or the twelfth row of the consistent mass matrix in Equation (3.23).

In HRZ lumping, named after Hinton, Rock, and Zienkiewicz [30], the consistent mass matrix is approximated by removing all off-diagonal terms and scaling the diagonal terms in a way that preserves the total mass of the element. The diagonal terms are scaled in groups that correspond to a direction in the local coordinate system. In each group all diagonal terms are scaled with the sum of the diagonal terms corresponding to translational degrees of freedom. This results in a diagonal matrix where the terms corresponding to translational degrees of freedom are $m / 2$ and all terms corresponding to rotational degrees of freedom are positive.

In Particle lumping [11] a point mass of $m / 2$ is placed at each node. This makes the diagonal terms that corresponds to translational degrees of freedom equal to $m / 2$. Those corresponding to rotational inertia can be set to zero, or can be derived from the inertia of the element. By concentrating the mass of an element at its nodes, the rotational inertia is overestimated. Therefore, the most accurate representation of the element's inertia would have negative terms for the rotational inertia. 
Row-sum lumping and Particle lumping with rotational inertia result in matrices with negative terms on the diagonal, making the mass matrix indefinite. Numerical experiments with the HHT- $\alpha$ method showed that negative terms on the diagonal of the mass matrix give problems with convergence. Because positive rotational inertia terms, such as those resulting from HRZ lumping, worsen the overestimation of rotational inertia, the most accurate results are obtained from a lumped mass matrix with zero rotational inertia.

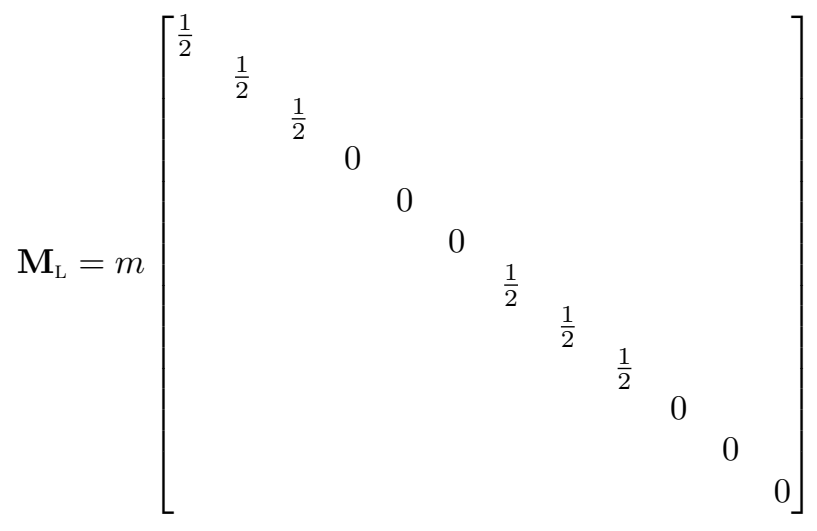

This lumped mass matrix has identical terms for the translational directions and zero terms for the rotational directions. When this matrix is rotated, the resulting matrix in the global coordinate system is identical to that in the local coordinate system.

\subsubsection{Comparison}

To analyse the difference between the consistent and the lumped mass matrix, the cantilevered pipe is revisited. Natural frequencies of this pipe are determined by two methods and the resulting frequencies are compared to an analytical solution. Furthermore, the effect of lumping the mass matrix on the required number of iterations and calculation time of the HHT- $\alpha$ method is investigated.

In the two methods to determine the natural frequencies the pipe is divided into ten elements. The properties of the pipe are given in Table 3.4. Both methods are applied using two mass matrices: a consistent mass matrix and a lumped mass matrix.

In the first method to determine the natural frequencies an eigenvalue problem is composed:

$$
\operatorname{det}\left(\mathbf{K}_{\mathrm{L}}^{\mathrm{sys}}-\omega^{2} \mathbf{M}_{\mathrm{L}}^{\mathrm{sys}}\right)=0
$$

This equation is solved for the eigenvalues $\omega$, from which the natural frequencies are found as $f=\omega / 2 \pi$. The superscript 'sys' indicates that these local matrices 
are assembled into a system matrix. Table 3.5 shows the pipe's natural frequencies; those indicated with 'eig' result from the eigenvalue problem.

In the second method to determine the natural frequencies, a fast Fourier transform algorithm [12] is applied to the total displacements of the pipe's free end. The displacements of the pipe in the time domain are obtained from a transient analysis using the HHT- $\alpha$ method with $\alpha=0.03$. A time interval of $7000 \mathrm{~s}$ is simulated using time steps of $\Delta t=1 \mathrm{~s}$. At the end of the first time step, two forces are applied to the pipe tip, as can be seen in Figure 3.3. These forces are equal to $F_{y}=5 \mathrm{kN}$ and $F_{z}=10 \mathrm{kN}$ and are removed at the end of the second time step. Results of simulations using the consistent and lumped mass matrices presented in Table 3.5, indicated with 'fft'.

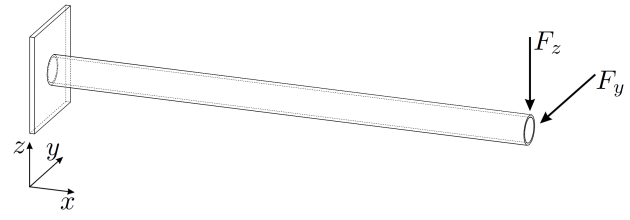

Figure 3.3: Cantilevered pipe loaded with two forces.
Table 3.4: Pipe properties.

\begin{tabular}{lll}
\hline Length & $L$ & $500 \mathrm{~m}$ \\
Diameter & $D$ & $559 \mathrm{~mm}(22$ inch $)$ \\
Wall thickness & $t$ & $21 \mathrm{~mm}$ \\
Young's modulus & $E$ & $207 \mathrm{GPa}$ \\
Poisson's ratio & $\nu$ & 0.3 \\
\hline
\end{tabular}

Table 3.5 also shows analytically calculated natural frequencies. These can be obtained in $\mathrm{Hz}$ from [38]:

$$
f_{i}=\frac{\left(B_{i} L_{0}\right)^{2}}{2 \pi L_{0}^{2}} \sqrt{\frac{E I}{\rho A}}
$$

Here $B_{i} L_{0}$ needs to be solved from a transcendental function, the first four solutions of which are $B_{1} L_{0}=1.875, B_{2} L_{0}=4.694, B_{3} L_{0}=7.855$ and $B_{4} L_{0}=10.996$.

Considering the consistent mass matrix, the natural frequencies obtained from the eigenvalue problem approximate the analytical solution very well. The natural frequencies obtained from the Fourier transform are a little lower than the analytically obtained frequencies, due to the numerical damping.

The lumped mass matrix overestimates the moment of inertia of an element that rotates around its centre, resulting in slightly lower natural frequencies than those obtained with the consistent mass matrix. The first natural frequencies obtained with the lumped mass matrix approximate very well the frequencies obtained with the consistent mass matrix.

Calculation times and the number of iterations using the HHT- $\alpha$ method with the different mass matrices are compared in Table 3.6. These are obtained using an effective stiffness matrix that does not include the derivative of the mass matrix. It can be seen that when the non-constant consistent mass matrix is used, the number of iterations is higher. When the derivative of the mass matrix is included, 
as shown in Equation (3.7), the number of iterations can be reduced. Computing this additional term would increase the computational costs per iteration.

The calculation time using the lumped mass matrix is approximately $10 \%$ shorter than the calculation time using the consistent mass matrix. This difference can be attributed partly to the difference in number of iterations and partly to lower computational costs per iteration.

It can be concluded that choosing the lumped mass matrix has a slight influence on the frequency of the result. The lumped mass matrix results in a constant system mass matrix, which only needs to be computed once. Using the consistent mass matrix requires an additional term in the effective stiffness matrix, increasing the computational time per iteration. Without this additional term the number of iterations increases. For efficiency the lumped mass matrix is selected for the pipe-lay model.

Table 3.5: Natural frequencies in $\mathrm{mHz}$; 'eig' indicates frequencies determined by solving an eigenvalue problem and ' $\mathrm{fft}$ ' indicates frequencies determined by applying a fast Fourier transform algorithm to the results of the HHT- $\alpha$ time integration method.

\begin{tabular}{|c|c|c|c|c|c|}
\hline & \multirow{2}{*}{ Analytical } & \multicolumn{2}{|c|}{ Consistent } & \multicolumn{2}{|c|}{ Lumped } \\
\hline & & eig & $\mathrm{fft}$ & eig & $\mathrm{fft}$ \\
\hline$\overline{f_{1}(\mathrm{r}}$ & 2.2 & 2.2 & 2.2 & 2.2 & 2.2 \\
\hline$f_{2}(\mathrm{mHz})$ & 13.8 & 13.8 & 13.8 & 13.6 & 13.6 \\
\hline$f_{3}(\mathrm{mHz})$ & 38.8 & 38.8 & 38.5 & 37.8 & 37.5 \\
\hline$f_{4}(\mathrm{mHz})$ & 76.0 & 76.0 & 74.5 & 73.3 & 71.9 \\
\hline
\end{tabular}

Table 3.6: Computational costs of the HHT- $\alpha$ method using consistent and lumped mass matrices.

\begin{tabular}{lll}
\hline & Number of iterations & Calculation time \\
\hline Consistent & 15221 & $8.1 \mathrm{~s}$ \\
Lumped & 14008 & $7.3 \mathrm{~s}$ \\
\hline
\end{tabular}

\subsection{Initialisation}

At the start of a dynamic pipe-lay simulation a stationary pipe is suddenly subject to a load. A sudden load can excite the high natural frequencies of the pipe. These vibrations are damped by numerical or structural damping after a period of time. When large time steps are chosen, the model cannot capture high frequency 
vibrations. This results in large changes in the state of the structure in each time increment, which can cause convergence problems.

This issue is illustrated by an example of a cantilevered pipe, which is given in Figure 3.4. This pipe is subject to an axial force of $N=30 \mathrm{kN}$; the properties of the pipe are given in Table 3.4. This example is simulated using the HHT- $\alpha$ method with $\alpha=0.03$ and time steps of $\Delta t=1 \mathrm{~s}$. At the end of the first time step, the axial force is suddenly applied, resulting in a high frequency vibration. The first axial natural frequency is equal to $2.6 \mathrm{~Hz}$; the period of this frequency is smaller than the time step. The result is a large change in reaction force at the end of each time increment, as can be seen in Figure 3.5. Due to numerical damping the high-frequency behaviour is damped over time, which shows that the high-frequency behaviour is a start-up problem. These large changes can cause convergence problems.

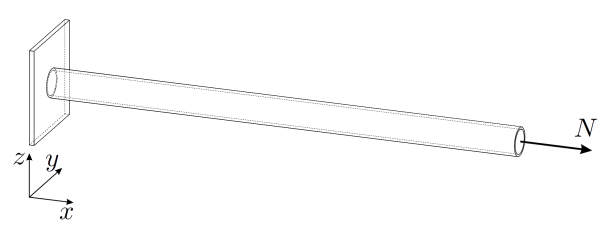

Figure 3.4: A cantilevered pipe with an axial load.

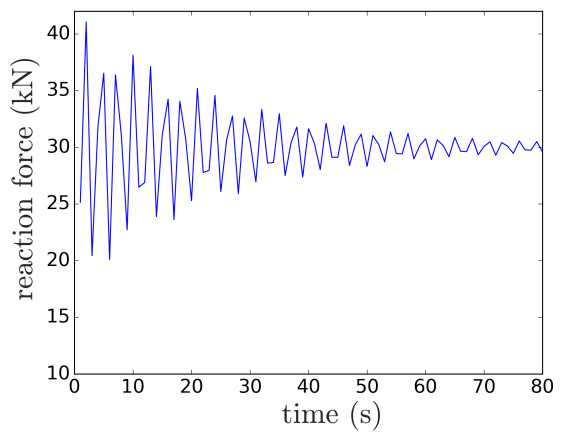

Figure 3.5: Reaction force of a cantilevered beam loaded with an axial force.

A simple initialisation procedure can solve this start-up issue. Using the HHT$\alpha$ method for numerical time integration, the parameter $\alpha$ can be used to tune the numerical damping in the model. This parameter determines the Newmark- $\beta$ parameters, which influence the nodal velocities and accelerations at the end of the time increment, as shown in Equation (3.3). In this initialisation procedure the Newmark- $\beta$ parameters are determined from initialisation parameter $\alpha_{\text {ini }}$ instead of $\alpha$ :

$$
\beta=\frac{\left(1+\alpha_{\text {ini }}\right)^{2}}{4} \quad \gamma=\frac{1}{2}+\alpha_{\text {ini }}
$$

At the start of a simulation the initialisation parameter can be set to $\alpha_{\text {ini }}=1$, such that the high-frequency behaviour is damped quickly. Hereafter, the parameter is linearly decreased to $\alpha$ during an initialisation period. The initial $\alpha_{\text {ini }}=1$ is not directly changed to $\alpha$, because this would also be a sudden change and can also excite a new high frequency vibration. Using this procedure, the initial high 
frequency vibration can be damped and there is no sudden change that causes a new high frequency vibration.

The example of the cantilevered pipe with an axial load is repeated with the initialisation procedure using an initialisation period of $20 \mathrm{~s}$. Figure 3.6 shows the value of $\alpha_{\text {ini }}$ during the simulated time. Figure 3.7 shows the reaction force of the cantilevered beam. Due to the initialisation procedure, the size of the reaction force is equal in size to the load at the start of the simulation. It can be concluded that the initialisation procedure is a solution to the start-up issue.

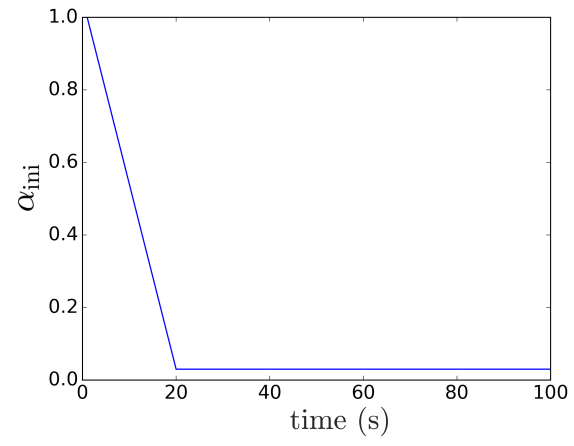

Figure 3.6: The initial $\alpha_{\text {ini }} \alpha_{\text {ini }}$ is decreased linearly from $\alpha_{\text {ini }}=1$ to $\alpha_{\text {ini }}=0.03$.

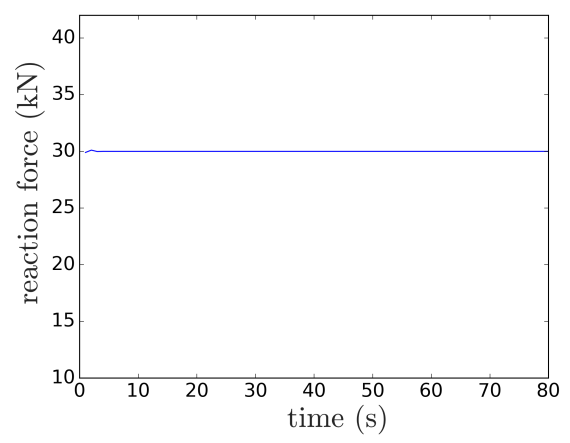

Figure 3.7: Reaction force of a cantilevered pipe loaded with an axial force using the initialisation procedure.

\subsection{Closure}

In this chapter the HHT- $\alpha$ method was selected as the time integration method best suited for the pipe-laying model. This method is second-order accurate and has controllable numerical damping that damps higher frequencies sufficiently without affecting the lower frequencies too much. The stability of this method is not dependent on the time step size. The HHT- $\alpha$ method can suffer from overshoot at the start of a simulation.

After comparing a consistent mass matrix and a lumped mass matrix, the lumped mass matrix was selected for the efficient pipe-lay mode. The lumped mass matrix is an approximation of a consistent mass matrix and is constant, therefore it needs to be computed only once. Using a lumped mass matrix also reduces computational costs because a non-constant mass matrix requires an additional term in the equivalent stiffness matrix. Results of the HHT- $\alpha$ method with a lumped mass matrix show slightly lower natural frequencies.

The number of natural frequencies in a model is equal to the number of degrees of freedom. A numerical model can include high frequencies that are physically 
not relevant. At the start of a numerical simulation a load is suddenly applied to the structure, which can excite frequencies that have a period shorter than the time step. This can result in incorrect predictions of displacements, velocities and accelerations of the structure. An initialisation procedure for the HHT- $\alpha$ method was presented that damps these high frequencies at the start of a simulation. 



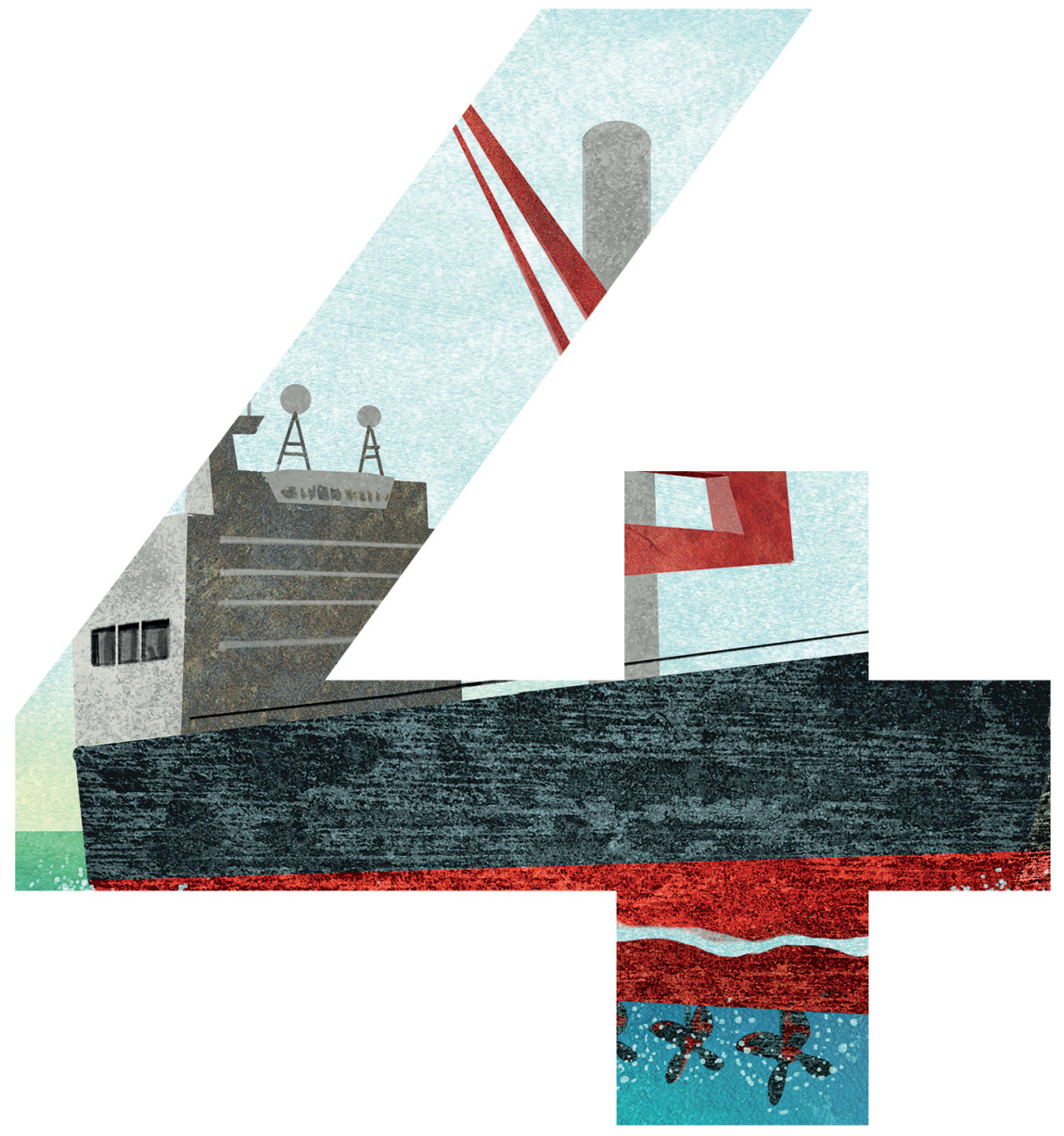




\section{4}

\section{Contact modelling}

During pipe-laying the pipe is in contact with the seabed and with roller-boxes on the stinger and on the vessel. These roller-boxes move due to the rotations and translations of the vessel. In this chapter a method for contact in finite element models is selected from literature and contact bodies for the seabed and rollerboxes are defined.

In Chapter 2 a formulation for co-rotational beam elements in static simulations was presented. In the contact method a contact force is exerted on a node of a co-rotational beam element. The contact method can also be applied in dynamic simulations, using the time integration method presented in Chapter 3. The contact method can be generally applied to beam elements, while the contact bodies are specifically defined for the pipe-lay model.

In finite element simulations contact is a boundary non-linearity. Different methods for boundary non-linearities in finite element simulations can be found in literature [59]. One of the most frequently used methods to account for boundary non-linearities is the Penalty method, which originates from constrained optimisation. Background and discussions on the Penalty method can be found in [43] for optimisation problems and in [34] for finite element simulations.

The Penalty method can be interpreted physically as a spring that is embedded in a contact body. In this method a binary contact-state is defined: the pipe is either in contact or it is not in contact with the body. When there is no contact there is no contact force. When there is contact a force is exerted on the pipe. The magnitude of this contact force is determined by a penalty parameter and by the distance the pipe has penetrated the interface of the contact body. When a linear penalty function is chosen the relation between the contact forces and the penetrated distance is linear, except when the contact-state changes.

Advantages of the Penalty method are its simplicity and the physical 
interpretation of the penalty function. Furthermore, this method is conservative, there is no artificial increase in energy. A drawback of the Penalty method is that convergence can be hindered because the contact force is not differentiable at the interface. However, changes in contact-state do not occur often in pipe-lay simulations. Another disadvantage is that a piercing distance is required to obtain a contact force. This is not a problem for the pipe-lay model, however, because the seabed and the roller-boxes are physically not rigid. Therefore, the Penalty method is an adequate contact method for the pipe-lay model.

The Penalty method is explained in more detail in Section 4.1. In the pipelay model this contact method needs to be applied at the seabed and at rollerboxes on the stinger and on the vessel. Modelling of the seabed is the subject of Section 4.2, modelling of the roller-boxes is the subject of Section 4.3. Because the contact force is not differentiable at the interface of a contact body, the required number of iterations can increase when the contact-state changes. Therefore, artificially alternating changes in contact-state need to be avoided. In Section 4.4 it is explained when artificially alternating changes in contact-state occur and a procedure to prevent these alternations is presented.

In the contact model a node is required at each point where the pipe is in contact with a roller-box. In the static simulation positions of the nodes can be chosen such that they coincide with the points of contact. In the dynamic simulation the pipe can move in axial direction over the roller-boxes due to the tensioner. In Section 4.5 a tensioner model is presented that models the paying-out and payingin of the pipe without moving the nodes of the pipe that are in contact with the roller-boxes. Paying-out and paying-in of the pipe when the axial tension at the tensioner exceeds a predefined upper or lower bound is accounted for in the pipelay model. When a new pipe section has been welded to the pipe in the firing line, the tensioner pays out the pipe and the vessel moves forward. This manoeuvre is not accounted for in this pipe-lay model.

\subsection{Penalty method}

In a finite element simulation the Penalty method can be interpreted physically as a non-linear spring element. This element is connected to a node of the pipe and will be referred to as a contact element. Because contact is modelled as an element, it is part of the internal forces in the equation of motion, Equation (3.10).

A contact force vector is defined at the node that the contact element has in common with the pipe. The direction of this contact force is determined by unit vector $\boldsymbol{n}$, which is normal to a contact surface. The size of this force is determined by a penalty factor $P$ and a gap $\Gamma-D / 2$. $\Gamma$ is the distance between the centre of the pipe and the interface and $D$ is the diameter of the pipe, as shown in Figure 4.1. The gap is the distance between the outer surface of the pipe and the interface of the contact body. There is contact when this distance is smaller than zero. The 
penalty factor can be seen as the stiffness of the spring that is embedded in the contact element. This defines the contact force vector as:

$$
\boldsymbol{F}^{\mathrm{c}}=P \boldsymbol{n} \max \left(\frac{D}{2}-\Gamma, 0\right)
$$

Equation (4.1) is not differentiable at the interface of the contact body, where the gap is equal to zero.

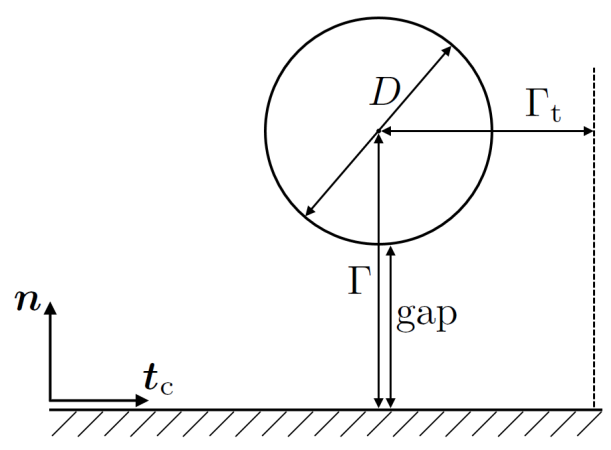

Figure 4.1: Schematic of a pipe near the interface of a contact body.

When the pipe is in contact with a body, displacements of the pipe in a plane tangentially to the contact surface are restricted by friction. Friction can be modelled using the Penalty method in combination with a stick-slip algorithm [18]. Stick means that the pipe does not move tangentially to the contact surface and slip means that the pipe does move in this plane.

At the seabed it is assumed that there is no slip when the pipe is in contact. At the roller-boxes it is assumed that friction can be disregarded. The basis of these assumptions are explained in Sections 4.2 and 4.3 respectively.

Friction without slip is implemented by applying an additional penalty force when the contact force is not zero. The direction of this friction force determined by $\boldsymbol{t}_{\mathrm{c}}$, which is a vector tangential to the contact body. The magnitude of the friction force is determined by a 'gap' $\Gamma_{\mathrm{t}}$ and a penalty factor $P_{\mathrm{t}} . \Gamma_{\mathrm{t}}$ is the distance from the centre of the pipe to a reference plane, as can be seen in Figure 4.1. $\Gamma_{\mathrm{t}}$ can be either positive or negative, resulting in a positive or negative friction force.

$$
\boldsymbol{F}_{\mathrm{t}}^{\mathrm{c}}=-P_{\mathrm{t}} \boldsymbol{t}_{\mathrm{c}} \Gamma_{\mathrm{t}}
$$

For all internal forces a contribution to the stiffness matrix is derived. The stiffness matrix is required for the Newton-Raphson algorithm, as presented in Section 2.3. Assuming that changes of $\boldsymbol{n}$ and $\boldsymbol{t}_{\mathrm{c}}$ can be disregarded in the derivation of the stiffness matrix, the contributions of the contact force and the friction force to the stiffness matrix are:

$$
\mathbf{K}^{\mathrm{c}}=P \boldsymbol{n} \boldsymbol{n}^{\mathrm{T}} \quad \mathbf{K}_{\mathrm{t}}^{\mathrm{c}}=-P_{\mathrm{t}} \boldsymbol{t}_{\mathrm{c}} \boldsymbol{t}_{\mathrm{c}}^{\mathrm{T}}
$$


In the following sections the contact surfaces and normal vectors $\boldsymbol{n}$ are defined at the seabed and at all roller-boxes. The tangential vector $\boldsymbol{t}_{\mathrm{c}}$ is defined at the seabed. It will be shown that the change of $\boldsymbol{n}$ and $\boldsymbol{t}_{\mathrm{c}}$ can indeed be disregarded in the stiffness matrix.

\subsection{Seabed}

The submerged end of the pipe is in contact with the seabed. This contact is modelled by the Penalty method, which accounts for the flexibility of the seabed. Reaction forces at the seabed are described by linear springs, the stiffness of these springs is set by the penalty factor.

The seabed can be seen as the interface of a contact body, thus the vector $\boldsymbol{n}$ is normal to the seabed. When the seabed is horizontal, $\boldsymbol{n}$ is directed in global $z$ direction. The seabed is stationary, thus the vector $\boldsymbol{n}$ is constant. The contact force is determined with Equation (4.1).

At the seabed friction is modelled using the Penalty method, assuming that there is no slip. In [18] contact of a pipe with a seabed was modelled with a stickslip algorithm for a J-lay simulation and it was shown that, at the seabed, the displacements tangential to the contact surface are small. Therefore, it is assumed that there is no slip when the pipe is in contact with the seabed.

When the pipe is in contact with the seabed, the friction force of Equation (4.2) is exerted on the pipe. The friction force in the axial direction of the pipe can be disregarded because the penalty factor $P_{\mathrm{t}}$ is smaller than the axial stiffness of the pipe and the displacements of the pipe's end that is in contact with the seabed are suppressed. The vector $\boldsymbol{t}_{\mathrm{c}}$ is in a direction tangential to the pipe and tangential to the seabed. The 'gap' $\Gamma_{\mathrm{t}}$ is defined as the distance between the centre of the pipe and the plane spanned by the global $x$ - and $z$-axis, such that the centre of the pipe lies on this plane when $\Gamma_{\mathrm{t}}$ is zero. Figure 4.2 shows the top view of a pipe on the seabed, with $\Gamma_{\mathrm{t}}$ indicated. Because the centre of the pipe lies approximately on the $x-z$ plane of the global coordinate system, $\boldsymbol{t}_{\mathrm{c}}$ can be set in a constant direction equal to the global $y$-direction.

At the seabed the local $x_{\mathrm{L}}$-axis of the pipe can still be at a small angle with the global $x$-axis because the 'gap' $\Gamma_{\mathrm{t}}$ is not exactly equal at each node. This results in a small friction force in axial direction of the pipe. This is not an issue because the component of $P_{\mathrm{t}} \boldsymbol{t}_{\mathrm{c}}$ in local $x_{\mathrm{L}}$-direction is much smaller than the axial stiffness.

\subsection{Roller-boxes}

On the stinger and on the vessel the pipe is supported by roller-boxes. A rollerbox contains several rows of parallel rollers. For different roller-boxes the shape 


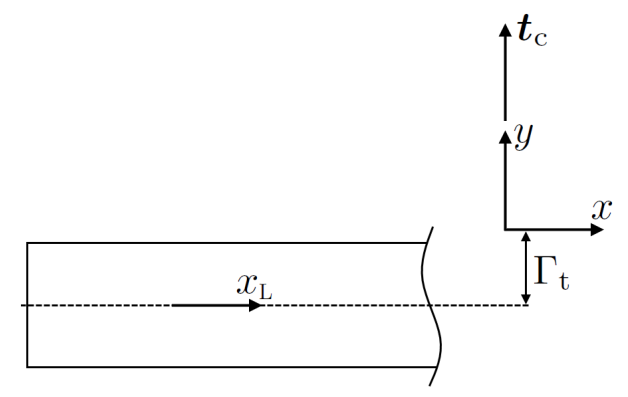

Figure 4.2: Top view of a pipe on the seabed.

of roller rows can be different. Figure 4.3 shows a pipe supported by V-shaped roller-boxes on a stinger, which are also used in the roller-box model.

For each roller-box contact is modelled with two contact elements that have one node in common with each other and the pipe. Hence, each roller-box exerts a single contact force at a predefined position on the pipe. In the discretisation of the pipe it must be ensured that the pipe model has a node at the position of each roller-box.

Figure 4.4 shows the V-shape of a roller-box. The pipe can be in contact with both sides of the $\mathrm{V}$-shape or with one of the sides. The distances between the contact areas and the centre of the pipe are $\Gamma_{1}$ and $\Gamma_{2}$. When $\Gamma_{i}$ is smaller than the radius of the pipe, there is contact.

At each roller-box the pipe is generally supported by two contact surfaces that are not parallel. Because the friction force is much smaller than the contact force, friction can be disregarded at the roller-boxes. Therefore, the tangential forces are equal to zero.

A roller-box translates and rotates due to displacements and rotations of the vessel. Its motions are determined relative to the centre of gravity of the vessel, assuming a rigid connection. Within a roller-box, the rollers can rotate such that the rollers are perpendicular to the pipe's axis. When rotated, the rollers are positioned in frame $\mathbf{S}$, as can be seen in Figures 4.4 and 4.5 .

Roller frame $\mathbf{S}$ is determined by three unit vectors. $\boldsymbol{s}_{1}$ is in the direction of the pipe's axis at the node that is in contact with the roller-box. This direction is determined by the first vector of nodal frame $\mathbf{T}$, as was defined in Section 2.1. The second vector of the roller-box frame $\boldsymbol{s}_{2}$ is perpendicular to the $z_{\mathrm{V}}$-direction of the vessel's coordinate system. It can be determined with vector $\boldsymbol{k}_{\mathrm{V}}$, which points in the $z_{\mathrm{V}}$-direction of the vessel's coordinate system, as shown in Figure 4.5. The third vector of the roller-box frame $s_{3}$ is simply perpendicular to the first 


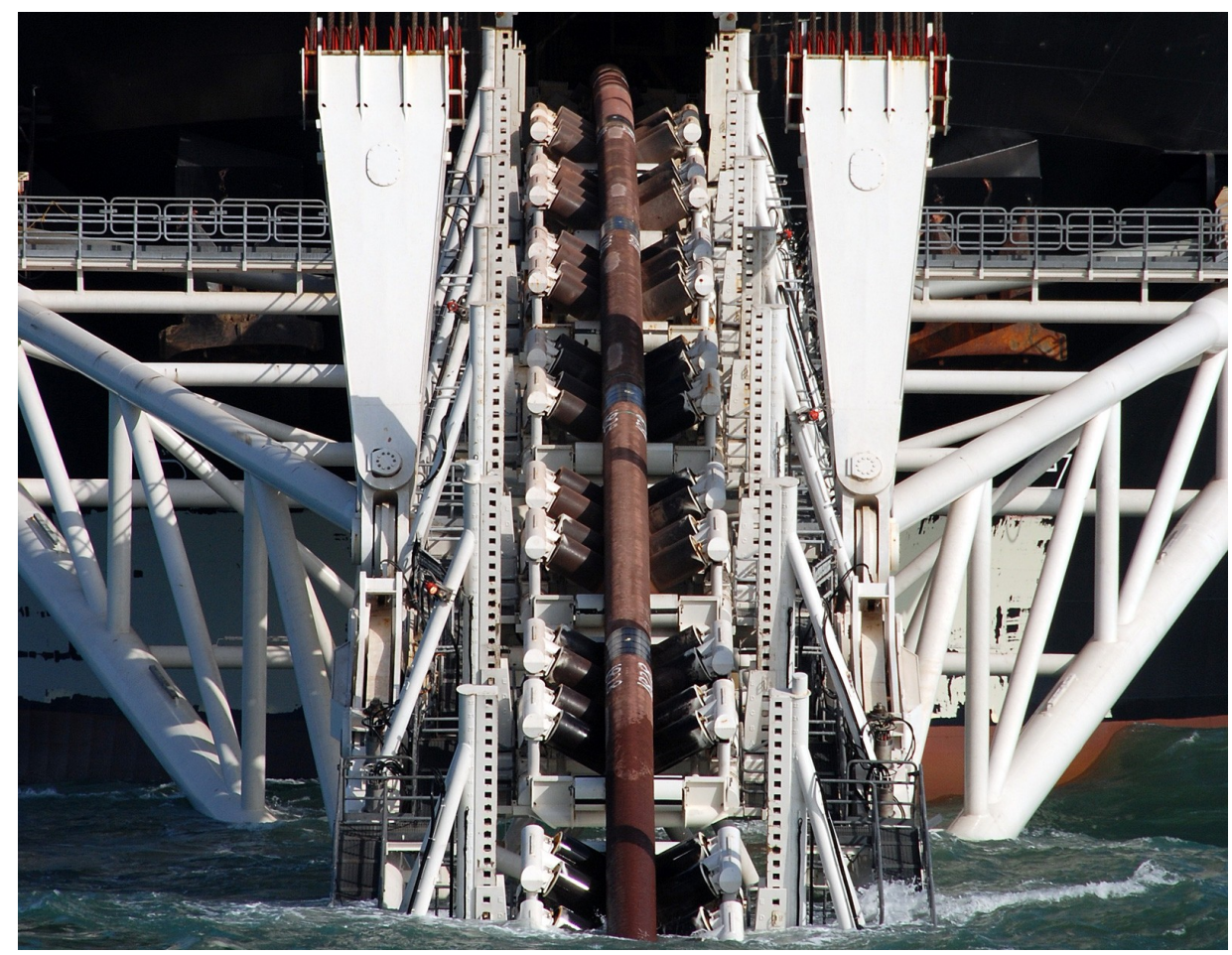

Figure 4.3: A pipe supported by roller-boxes on a stinger [2].

and the second vector. The three vectors of $\mathbf{S}$ are:

$$
\boldsymbol{s}_{1}=\boldsymbol{t}_{1} \quad \boldsymbol{s}_{2}=\frac{\boldsymbol{s}_{1} \times \boldsymbol{k}_{\mathrm{V}}}{\left\|\boldsymbol{s}_{1} \times \boldsymbol{k}_{\mathrm{V}}\right\|} \quad \boldsymbol{s}_{3}=\boldsymbol{s}_{1} \times \boldsymbol{s}_{2}
$$

The vectors $\boldsymbol{n}_{i}$, which determine the direction of the contact force, are defined in the $\boldsymbol{s}_{2}-\boldsymbol{s}_{3}$ plane of roller frame $\mathbf{S}$. Figure 4.4 shows two normal vectors $\boldsymbol{n}_{i}$ in this plane because there are two contact surfaces. These vectors can be determined from trigonometry:

$$
\begin{aligned}
& \boldsymbol{n}_{1}=\boldsymbol{s}_{2} \sin \varphi+\boldsymbol{s}_{3} \cos \varphi \\
& \boldsymbol{n}_{2}=-\boldsymbol{s}_{2} \sin \varphi+\boldsymbol{s}_{3} \cos \varphi
\end{aligned}
$$

In the derivation of the stiffness matrix due to the Penalty method, Equation (4.3), it is assumed that vectors $\boldsymbol{n}_{i}$ are constant.

Because the ship's movements are prescribed, the positions of roller-boxes do not vary over iterations. However, the roller-boxes can rotate due to the orientation of the pipe. In Equation (4.4) the orientation of the pipe is defined by vector $\boldsymbol{t}_{1}$. A stiffness matrix, which includes the variation of $\boldsymbol{t}_{1}$, was also determined. 


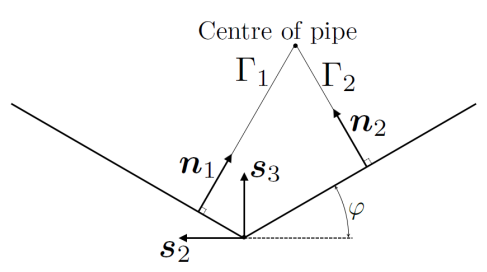

Figure 4.4: V-shape of a roller-box with normal vectors.

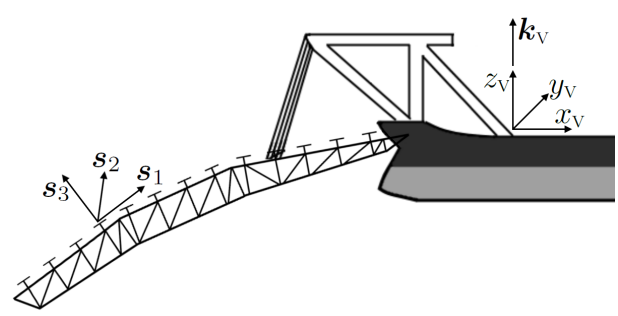

Figure 4.5: Roller-box frame and the vessel coordinate system.

Numerical experiments showed that this addition to the stiffness matrix had no influence on the convergence.

\subsection{Contact-state procedure}

At the end of a time step the contact-state is not necessarily equal to that at the beginning of the time step. During the iterative Newton-Raphson algorithm, which determines the configuration of the pipe at the end of the time step, artificially alternating changes of contact-state can occur. Because the contact force is not differentiable at the interface of the contact body, artificially alternating changes of contact-state increase the number of iterations and can even cause divergence. In this section is explained when artificially alternating changes of contact-state occur and a procedure to prevent these changes is presented.

Artificially alternating changes in contact-state occur when, for example, rollerboxes are moved downward by a prescribed displacement. In the first iteration of a time increment the roller-boxes have moved but the pipe has not. Therefore, the pipe is not in contact with the stinger in the first iteration. Due to gravity loads the pipe penetrates through the roller-boxes, which results in a very large gap in the second iteration. This results in a contact-state change from 'no contact' to 'contact' and a very large contact force. Hence, when roller-boxes are moved downward, the contact-state of nodes near the stinger changes back and forth in the first iterations.

Artificially alternating changes in contact state can also occur when roller-boxes are moved upward over a large distance. When the time steps are large the prescribed displacements of the roller-boxes can also be large. In the first iteration of a time increment the roller-boxes have moved but the pipe has not. Therefore, there is a large gap and a large contact force in the first iteration. Due to this large contact force the pipe moves upward. Because of the geometric non-linearity, the pipe can move too far upward. When this happens, the 
contact-state changes to 'no contact' in the second iteration and the pipe penetrates through the roller-boxes due to gravity loads. In the third iteration the contact-state changes to 'contact' and the contact force becomes large. Hence, when roller-boxes are moved over large prescribed displacements, the contact-state of nodes near the stinger changes back and forth.

Algorithm 4.1 shows an implementation of a contact-state check that is executed in every iteration before determining the contact forces. It shows that the contactstate can change every time the algorithm is executed. Using this algorithm, artificially alternating changes in contact-state occur.

A dedicated contact-state procedure can prevent these artificial alternations. The idea behind this procedure is that the change in contact-state from 'contact' to 'no contact' is delayed by one iteration. Changes of contact-state from 'no contact' to 'contact' are immediately enforced. As can be seen in Algorithm 4.2, this is implemented by allowing the contact-state to change to 'no contact' only when two consecutive iterations predict that the contact-state has to change to 'no contact'. A result of this procedure is that when the roller-boxes move downward, the nodes that were in contact with the stinger remain in contact. This can result in a negative contact force that pulls the pipe back to the roller-boxes. In the second iteration the pipe is back in contact with the roller-boxes. This procedure makes sure that there are no artificially alternating changes of contact-state.

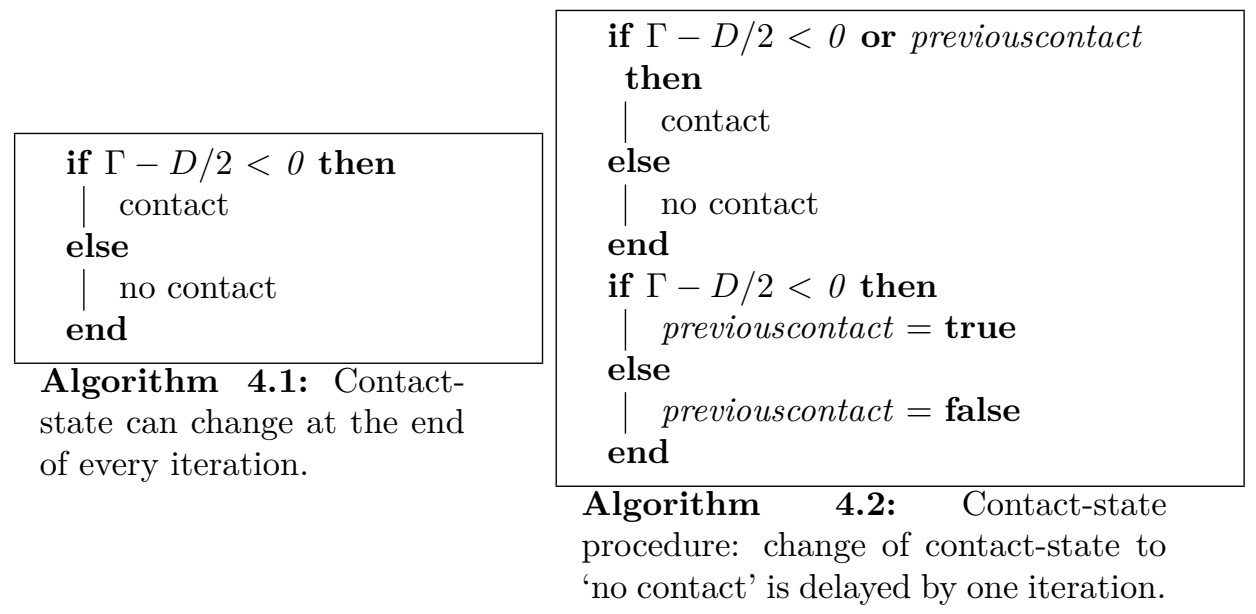

The necessity and effect of this contact-state procedure is shown with the convergence of a representative time increment from an S-lay simulation. In this time increment the roller-boxes move upward. Figure 4.6 shows the convergence of this increment with and without applying the contact-state procedure. This convergence is determined by a force-based error norm in each iteration, as shown in Equation (2.48).

Without the contact-state procedure the contact-state of most roller-boxes are 


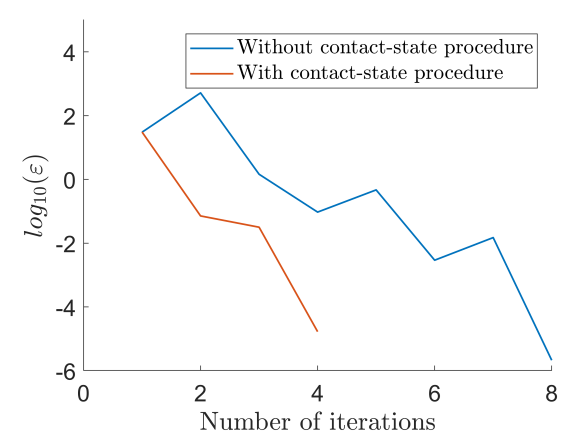

Figure 4.6: Convergence plot of a representative time increment of an S-lay simulation.

changed to 'no contact' in the first iteration. This results in a smaller error than that obtained with the contact-state procedure but this is hardly noticeable because the error is dominated by large axial forces due to the high axial stiffness. At the end of this iteration the pipe penetrates through the roller-boxes. In the second iteration the contact-state changes again, resulting in large contact forces and a large error in the second iteration. In the third iteration the error finally reduces. The remaining iterations still have some change of contact-state due to large displacements of the pipe, resulting in two additional error peaks. Without the contact-state procedure there are multiple error peaks from contact-state changes, resulting in slow convergence.

When the contact-state procedure shown in Algorithm 4.2 is applied, the number of iterations reduces from 8 to 4 . In the first iteration the error is large due to the large axial forces. In the second iteration the error immediately decreases. With this procedure there are no error peaks from artificially alternating changes of contact-state changes, resulting in good convergence.

\subsection{Tensioner}

On board an S-lay vessel one or more tensioners are present at the end of the firing line. They apply tension on the pipeline while it is being installed on the seabed. The tensioners can pay out or pay in the pipe when the axial tension exceeds predefined upper or lower bounds to prevent excessive deformations in the sagbend. The domain within these bounds is called the deadband. This section presents a basic model of a tensioner that can pay out and pay in the pipe when the axial force is not within the deadband. A tensioner can pay-out the pipe after a new pipe segment has been welded on, but this is not part of the pipe-lay model.

The tensioner measures the axial tension and controls the tension by paying the 
pipe in or out. In the tensioner model these two functions are separated. The measuring function is modelled on the first node of the pipe, which is on board the vessel. Tensioner forces are the forces at this node. Paying in and out is modelled by increasing or decreasing the length of a dedicated tensioner element. To make sure that the nodes on the stinger remain on the roller-boxes, the first element after the stinger is chosen as the dedicated tensioner element.

Paying in and out of the pipe is simplified to a continuous function instead of only paying in and out when the limit is exceeded. It is assumed that the change in axial tension can be linearly related to the displacement of the stinger tip. This is a valid approximation when the water is deep, in which case the change in axial tension is dominated by the inertia of the pipe. The accelerations of the pipe in axial direction are linearly dependent on the displacements in axial direction, as can be seen in Equation (3.3). When it is assumed that the displacements in axial direction of the pipe are approximately equal to the displacement of the stinger tip in the direction of the pipe, the change in axial force can be related to the displacement of the stinger tip.

In the tensioner model a static tension $T_{0}$ and a deadband tension $T_{\mathrm{d}}$ are defined. The upper and lower bounds of the tension are calculated by:

$$
T^{\text {upper }}=T_{0}+T_{\mathrm{d}} \quad T^{\text {lower }}=T_{0}-T_{\mathrm{d}}
$$

The increment of the dedicated tensioner element's increase or decrease, $\Delta u_{\mathrm{t}}$, is determined by the displacement increment of the stinger tip multiplied by a tensioner factor $f_{\mathrm{t}}$.

$$
\Delta u_{\mathrm{t}}=\Delta u_{\mathrm{st}} f_{\mathrm{t}}
$$

Here, $\Delta u_{\text {st }}$ is the displacement increment of the stinger tip in the direction of the pipe. Initially, the tensioner factor is zero. When the deadband is exceeded in an increment, the factor is increased.

$$
\Delta f_{\mathrm{t}}=f_{0} \frac{\left|R_{\mathrm{t}}-T_{0}\right|-T_{\mathrm{d}}}{\left|R_{\mathrm{t}}-T_{0}\right|}
$$

Here, $R_{\mathrm{t}}$ is the reaction force at the tensioner node and $f_{0}$ is a factor between zero and one that effectively damps the increase of the tensioner factor.

This tensioner model is demonstrated by an example of a tensioner in an S-lay simulation, where the vessel responds to a regular wave. In this example the static result shows an axial force at the tensioner of $T_{0}=2843 \mathrm{kN}$. The deadband is set to $15 \%$ of $T_{0}$. Figure 4.7 shows the reaction force at the tensioner resulting from a dynamic simulation with the tensioner deactivated. It can be seen that the tension exceeds the deadband when the tensioner is deactivated.

When the tensioner is activated the reaction force at the tensioner decreases during the dynamic simulation, until it is within the deadband limits. Figure 4.8a shows the reaction force at the tensioner when $f_{0}=1$. It can be seen that the reaction force is reduced more than required. Figure $4.8 \mathrm{~b}$ shows the reaction force at the 
tensioner when $f_{0}=0.1$. With this tensioner model setting, the reaction forces are outside the deadband for a longer period but they are not reduced too much.

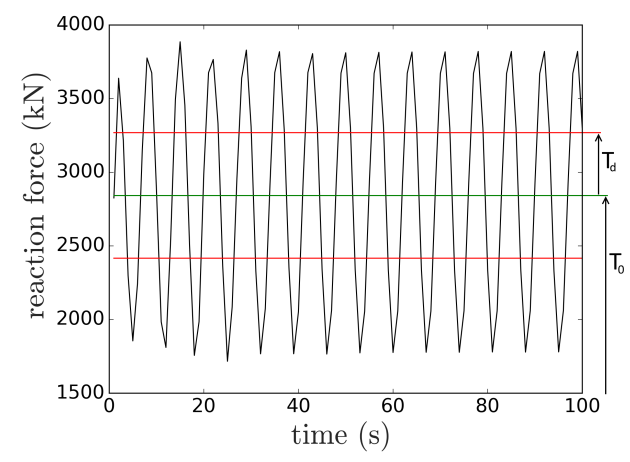

Figure 4.7: Total reaction force at tensioner, tensioner deactivated: $f_{0}=0$.

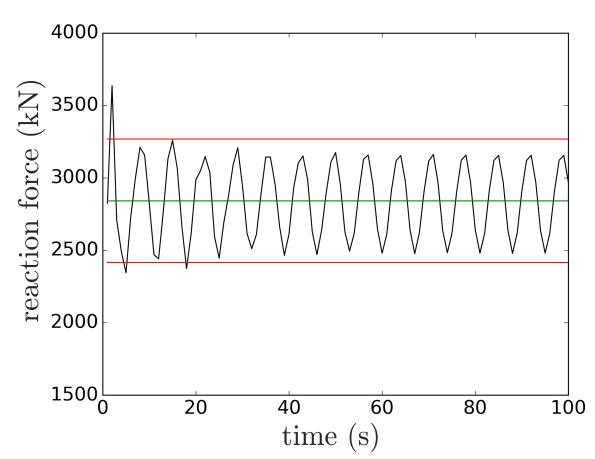

(a) $f_{0}=1$.

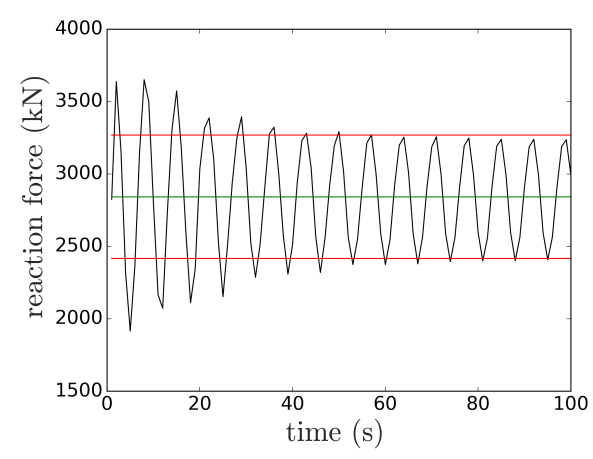

(b) $f_{0}=0.1$.

Figure 4.8: Total reaction forces at tensioner.

The tensioner model presented in this section is a basic tensioner model that has limited applicability. This tensioner model acts continuously, which means that when a generic wave is used the tensioner is paying in and out during small amplitude waves as well. In reality the tensioner acts only during the period where the deadband is exceeded. Furthermore, it is assumed that the reaction forces in the tensioner are dominated by the inertia of the pipe. By increasing and decreasing the length of the dedicated pipe element with respect to the displacements of the stinger tip, the accelerations of the pipe can be reduced. In shallow water, where the pipe leaves the ship at a small angle, the reaction forces are not dominated by the inertia of the pipe. Because of these limitations it is recommended that this tensioner model be replaced with a more accurate 
tensioner model in future research.

\subsection{Closure}

In the pipe-lay model the pipe can be in contact with the seabed and with rollerboxes on the stinger and on the vessel. In this chapter the Penalty method was presented to model contact. Additionally, contact bodies that model the seabed and roller-boxes were presented.

The direction of the contact force is defined by a vector normal to the interface of the contact bodies. On the seabed this normal vector is perpendicular to the seabed. On the stinger V-shaped roller-boxes are in contact with the pipe. Each roller-box has two normal vectors, one for each side of the V-shape. The roller-box translates and rotates relative to the centre of gravity of the vessel.

It was shown that contact-state can alternate between 'contact' and 'no contact' over several iterations, which can hinder convergence. To prevent this alternating behaviour, a delayed contact-state procedure was introduced.

The contact forces are applied to a node of the co-rotational beam element at a fixed position and the nodes cannot move in axial direction on the stinger. To account for the axial movement due to the tensioner, a dedicated tensioner element was defined after the stinger. A basic tensioner model was presented and it was shown that this model reduced axial force variations in the pipe during an Slay simulation. For future research it is recommended that this basic model be replaced with a more detailed one. 



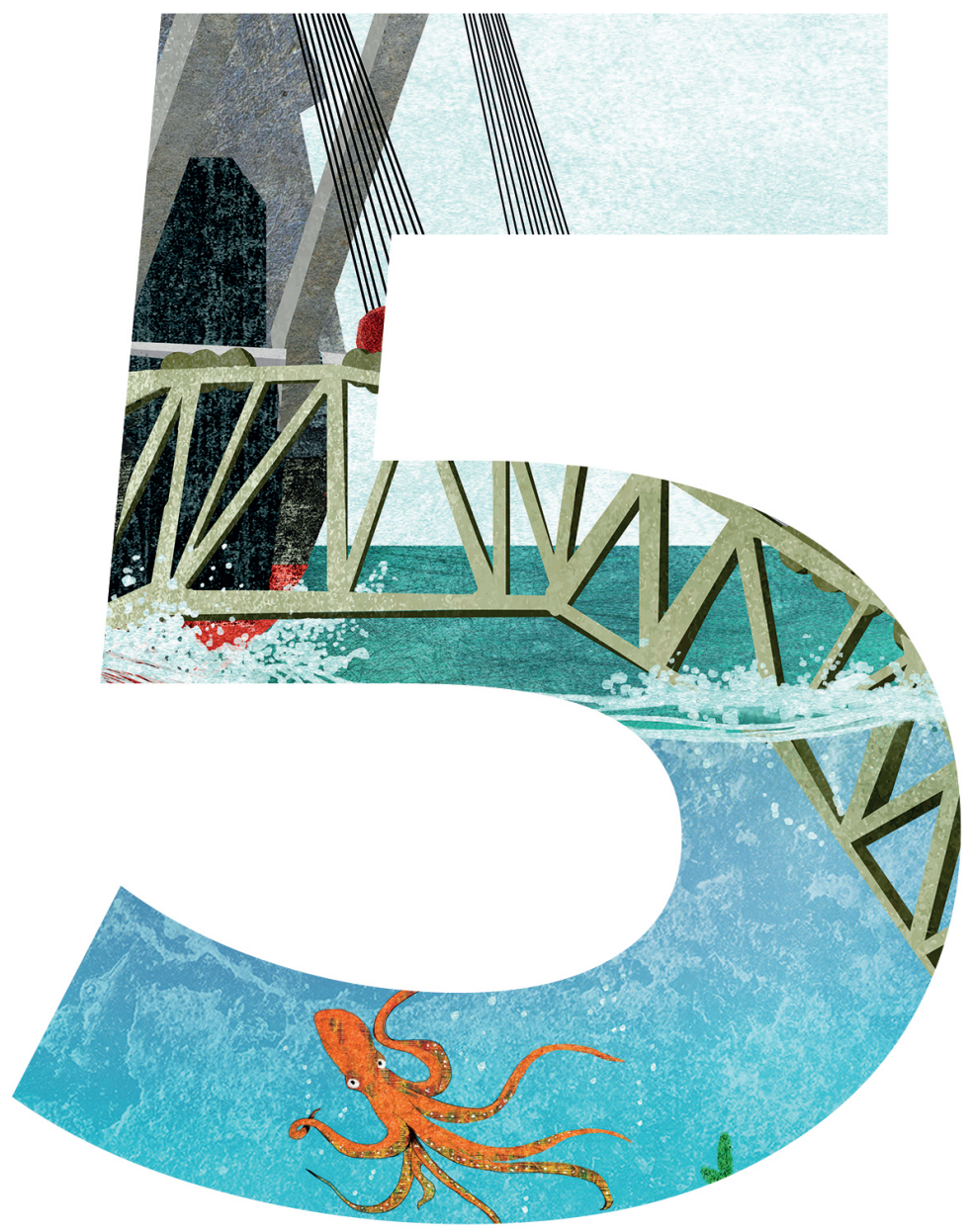




\section{5}

\section{Loads and prescribed displacements}

In the pipe-lay model displacements of the pipe are dependent on external loads, which include gravity, hydrostatic and hydrodynamic loads. Displacements of the pipe-laying vessel are prescribed by a response to waves at the water surface. It is assumed that the stinger is connected rigidly to the vessel, thus the displacements of the stinger and roller-boxes are also prescribed. Due to the contact between the pipe and the roller-boxes, motions of the pipe-laying vessel result directly in forces on the pipe. In this chapter external loads and prescribed displacements of the pipe-laying vessel are presented.

In Chapter 2 a basis for static simulations with co-rotational beam element, which account for geometric non-linearity, was established. In Section 2.4 distributed loads on these elements were presented, with gravity modelled as a distributed load. In Chapter 3 this basis was extended to dynamic simulations with a numerical time integration method. In this chapter the pipe-lay model is developed from this basis by adding sub-models for external loads. Additionally, the prescribed displacements of the pipe-laying vessel are described, resulting in prescribed displacement of the contact bodies for the roller-boxes, as presented in Chapter 4. At the end of this chapter a pipe-lay simulation with a linear-elastic material model can be performed. Plasticity will be dealt with in Chapter 6 .

Hydrostatic loads are a result of water pressure acting on the surface of the submerged pipe. These loads are commonly modelled by Archimedes' law, which calculates axial forces and axial strains incorrectly. In Section 5.1 a method to describe the correct buoyancy loads is presented. It is investigated when the model based on Archimedes' law is sufficient and when the detailed buoyancy model is required.

Hydrodynamic loads, which are presented in Section 5.2, are modelled by 
Morison's equation modified for a moving cylinder. This semi-empirical equation approximates hydrodynamic loads on cylindrical objects. The use of this equation is computationally inexpensive and, for this application, the approximation of the hydrodynamic loads is sufficiently accurate when using calibrated coefficients [52], unless vortex induced vibrations occur. The equation consists of three parts: loads due to drag, due to added mass and due to a pressure gradient. These loads, which are part of the external forces in the dynamic pipe-lay model, can be a function of the velocities and accelerations of the pipe.

In Section 5.3 surface waves and the vessel's response to these waves are presented. At sea the waves are influenced by environmental conditions such as the wind speed. These waves are often modelled by a superposition of waves within a frequency range, where the contribution of each frequency is determined by a wave spectrum. Different waves spectra exist, either for a specific sea or for a general application. The motions of the six degrees of freedom of a vessel's centre of gravity are related to the motion of the wave. When the amplitude of the wave is small in relation to its length and the angle at which the wave encounters the vessel is constant, this relation can be assumed linear. For each vessel and each encountering wave angle linear transfer parameters, which describe the motion of the vessel, can be determined. These linear parameters are used as an input in the pipe-lay model.

In Section 5.4 two considerations on the developed pipe-lay model are given. The first consideration on the pipe-lay model concerns the maximum allowed time step size. This time step is not dependent on stability or convergence, but on the frequencies of the vessel's response to the waves. The second consideration on the pipe-lay model concerns axial compression in submerged pipes. This axial compression, in combination with second-order terms in the calculation of the element's bending moments, can result in incorrect predictions of local rotations. An approach to getting correct predictions is presented.

\subsection{Hydrostatic loads}

When the pipe is submerged, a pressure acts on its outer surface resulting in hydrostatic loads. These loads cause an upthrust known as buoyancy. In pipe-lay models these loads are often approximated by determining a so-called submerged weight of each element. This submerged weight is determined by calculating an upthrust with Archimedes' law, which is subtracted from the gravity load on the pipe. This submerged weight model incorrectly assumes that the water pressure also acts on the cross-sectional surface at the ends of an element, resulting in an incorrect axial force and axial strain during computations of the pipe-lay model. A post-processing step can correct the axial forces and strains. This method is presented in Section 5.1.1. 
Yazdchi and Crisfield [66] presented a method for determining buoyancy loads on a submerged pipe that correctly determines the axial forces during computations of the pipe-lay model. In this method it is assumed that all elements are straight pipe sections that are connected to each other at an angle. The loads are determined by integrating the pressure distribution over the outer surface of the entire pipe string. In Section 5.1.2 a buoyancy model that augments the method of Yazdchi and Crisfield is presented. Equivalent moments have been added and a correction has been made to the cross-sectional surface at the connection of two elements.

In the submerged weight model the correct axial forces and strains are unknown during simulation. In the buoyancy model the correct forces are known. In Section 5.1.3 an investigation is made of when the submerged weight model gives sufficiently accurate results and when the more accurate buoyancy model must be used.

\subsubsection{Submerged weight}

In existing pipe-lay models a part of the hydrostatic loads are taken into account by determining a so-called submerged weight. The submerged weight $w$ is calculated by subtracting from a gravity load an upward force $q_{\mathrm{b}}$ resulting from Archimedes' law. The gravity load $q$ is simply a distributed load per unit length acting on the pipe due to gravity.

$$
\begin{aligned}
q & =\left(A_{\text {ext }}-A_{\text {in }}\right) \rho g \\
w & =q-q_{\mathrm{b}} \quad \text { where } \quad q_{\mathrm{b}}=\left(\rho_{\mathrm{w}} A_{\text {ext }}-\rho_{\text {in }} A_{\text {in }}\right) g
\end{aligned}
$$

Here, $A_{\text {ext }}$ and $A_{\text {in }}$ are the external and internal cross-sectional areas of the pipe. $\rho_{\mathrm{w}}$ is the density of the sea water, $\rho_{\text {in }}$ is the density of the fluid in the pipe and $g$ is the standard acceleration due to gravity.

On each submerged element $w$ is applied instead of the gravity load $q$, which can be interpreted as applying Archimedes' law on each individual element. This means that it is assumed that a pressure acts on all surfaces of the element, including the cross-sectional areas at the element's boundaries. This overestimates the axial force in submerged elements, which must be corrected in a post-processing step. A correct representation of the hydrostatic loads is obtained by subtracting an axial load from the results of Archimedes' law, as illustrated in Figure 5.1

The axial force resulting from the simulation is known as effective tension $N_{\text {eff }}$ [57]. The correct axial force $N_{\text {true }}$ is determined by correcting for the pressure loads on the cross-sectional surface:

$$
N_{\text {true }}=N_{\text {eff }}-p_{\text {ext }} A_{\text {ext }}+p_{\text {in }} A_{\text {in }}
$$

Here, $p_{\text {ext }}$ and $p_{\text {in }}$ are the external and internal pressure. These pressures are dependent on the water depth, thus the correction on the axial force is not constant over the length of an element. 

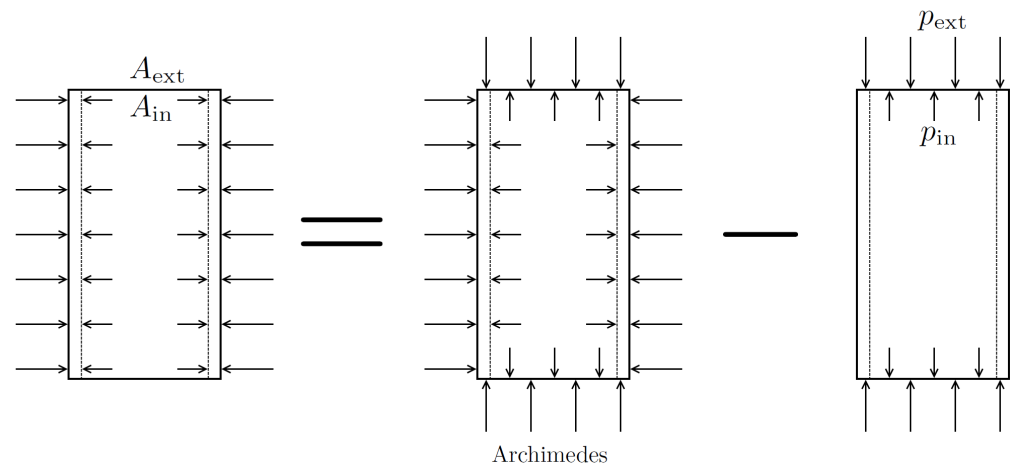

Figure 5.1: When the hydrostatic load on a pipe is represented by Archimedes' law, a correction on the axial force is required.

Because the correction on the axial forces is a post-processing step, the model is evaluated with an incorrect axial tension. In case of non-linear material behaviour the correct axial tension is required during processing and Equation (5.2) is insufficient.

\subsubsection{Buoyancy}

Yazdchi and Crisfield [66] presented a method for determining buoyancy loads on a submerged pipe. In this method a curved pipe is approximated by straight elements that are connected to each other at an angle, as can be seen in Figure 5.2 .
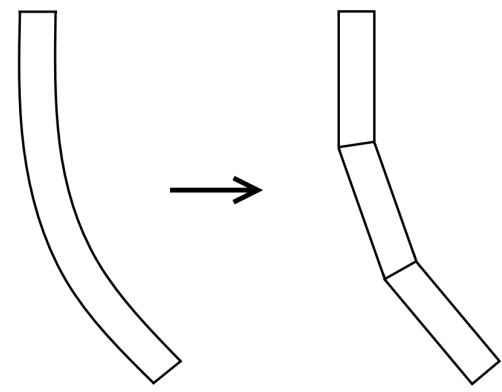

Figure 5.2: A curved pipe approximated by straight elements that are connected at an angle. 
By integrating the pressure analytically over the outer surface of the pipe, three contributions to the buoyancy forces are found. The first contribution is from the distributed pressure on the straight pipe element. The second contribution is from the surface area at the connection between two elements. At this connection, the surface area on the outer curve increases and the surface area on the inner curve decreases in relation to the straight element. This change in surface area represents the effect of the pipe's curvature. The third contribution is from a submerged pipe's closed end, which is referred to as a capped end.

The method presented here augments the method of Yazdchi and Crisfield on two aspects, see also De Vries et al. [18]. The first aspect is that the new method considers not only nodal forces but also nodal moments. These nodal forces and moments are equivalent to a distributed pressure load. The second aspect is that the cross-sectional areas at the nodes are not perpendicular to the pipe but at an angle determined by the local rotations of the element. In the new method the surfaces of these cross-sectional areas are determined exactly, while in the method of Yazdchi and Crisfield the cross-sectional areas are assumed perpendicular to the pipe.

The buoyancy method is presented in four sections. The first section presents the contribution due to the distributed pressure on the straight pipe, augmented with nodal moments. The second and third sections present the contributions of the pipe's curvature and capped ends as in the original method of Yazdchi and Crisfield. The fourth section presents the correction on the cross-sectional area.

The buoyancy load is an external force in the equation of motion, Equation (3.10) and contributes to the stiffness matrix. This contribution is derived in Appendix A.

\section{Distributed pressure}

A pressure field acts on the surface of the pipe. On a straight element the pressure can be integrated over the outer surface area of the pipe. This results in a distributed load over the length of the element. On a horizontal pipe section the distributed load due to the pressure distribution is equal to the upward load $q_{\mathrm{b}}$ shown in Equation (5.1). When the pipe is not horizontal, the pressure difference between the highest and lowest point of the pipe's cross-section is smaller than that used to calculate $q_{\mathrm{b}}$. The reduced distributed load can be calculated with:

$$
\bar{q}_{\mathrm{b}}=q_{\mathrm{b}} \boldsymbol{e}_{\mathrm{n}}^{T} \boldsymbol{k}
$$

Here, $\boldsymbol{k}$ is the unit vector in the global $z$-direction, as shown in Figure 5.3. $\boldsymbol{e}_{\mathrm{n}}$ is a unit vector normal to the local $x_{\mathrm{L}}$-axis and in the plane spanned by the local $x_{\mathrm{L}}$-axis and global $z$-direction:

$$
\boldsymbol{e}_{\mathrm{n}}=\frac{\left(\mathbf{I}-\boldsymbol{e}_{1} \boldsymbol{e}_{1}^{T}\right) \boldsymbol{k}}{\left\|\left(\mathbf{I}-\boldsymbol{e}_{1} \boldsymbol{e}_{1}^{T}\right) \boldsymbol{k}\right\|}
$$


Here, $\boldsymbol{e}_{1}$ is the vector in local $x_{\mathrm{L}}$-direction as shown in Section 2.1. The derivation of $\boldsymbol{e}_{\mathrm{n}}$ can be found in [66].

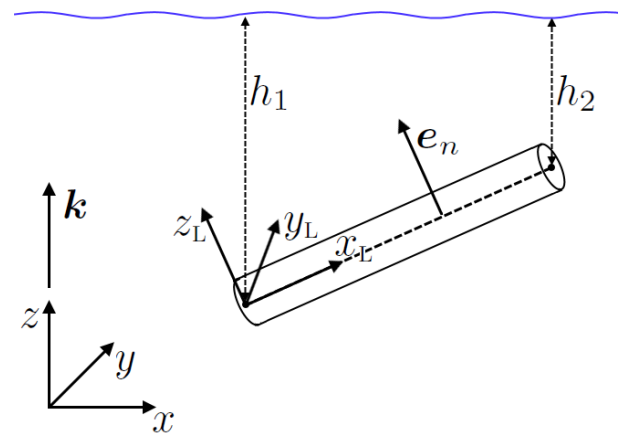

Figure 5.3: Schematic representation of a buoyancy element.

When the pipe is fully submerged, the equivalent nodal forces and moments can be determined from the distributed load, as described in Section 2.4. When an element is only partially submerged, a different approach is required. Equivalent forces and moments are scaled with a factor at each node. For the equivalent forces these factors are named $b_{1}$ and $b_{2}$ such that the magnitude of the nodal forces is equal to:

$$
F_{i}=b_{i} \bar{q}_{\mathrm{b}} L
$$

When the pipe is completely submerged, $b_{1}$ and $b_{2}$ are equal to $1 / 2$, which corresponds to the equivalent nodal forces described in Section 2.4. When only the first node is submerged, the parameters $b_{i}$ are determined as [66]:

$$
\begin{aligned}
b_{1} & =\bar{h}-b_{2} \\
b_{2} & =\frac{1}{2} \bar{h}^{2}
\end{aligned} \quad \text { where } \quad \bar{h}=\frac{h_{1}}{h_{1}-h_{2}}
$$

Here, distances $h_{1}$ and $h_{2}$ describe the positions of the first and second node with respect to the water surface, as can be seen in Figure 5.3. These distances are positive when the corresponding node is submerged and negative when the corresponding node is above water.

The distributed load in Equation (5.3) and the equivalent nodal forces in Equation (5.5) are according to the original buoyancy model of Yazdchi and Crisfield [66]. Here, equivalent moments are added, which are determined using factors $b_{\mathrm{m} 1}$ and $b_{\mathrm{m} 2}$ such that the magnitude of the nodal moments is equal to:

$$
M_{i}=b_{\mathrm{m} i} \bar{q}_{\mathrm{b}} L^{2}
$$

When the element is completely submerged, these factors are equal to $1 / 12$ and $-1 / 12$, which corresponds to the equivalent nodal moments described in Section 
2.4. This results in a nodal force vector that is statically and kinematically equivalent to the uniformly distributed load.

The nodal moments are determined from the work equivalence done by the distributed load. This work can be calculated by an integral over the submerged section of the pipe.

$$
W=\int_{0}^{a} \bar{q}_{\mathrm{b}} w_{\mathrm{L}} d x_{\mathrm{L}}
$$

Here $a$ is the distance between the first node and the water surface and $w_{\mathrm{L}}$ is the displacement in local $z_{\mathrm{L}}$-direction within the element. This displacement is determined by a cubic Hermite interpolation function, as shown in Section 2.1. The forces and moments corresponding to each degree of freedom can be determined by the derivative to these degrees of freedom. For the moment at the first node this results in:

$$
M_{1}=\int_{0}^{a} \bar{q}_{\mathrm{b}} \frac{\partial w_{\mathrm{L}}}{\partial \theta_{1}} d x_{\mathrm{L}}
$$

By solving this integral factors $b_{\mathrm{m} 1}$ and $b_{\mathrm{m} 2}$ are determined:

$$
\begin{aligned}
& b_{\mathrm{m} 1}=\frac{1}{4} \bar{h}^{4}-\frac{2}{3} \bar{h}^{3}+\frac{1}{2} \bar{h}^{2} \\
& b_{\mathrm{m} 2}=\frac{1}{4} \bar{h}^{4}-\frac{1}{3} \bar{h}^{3}
\end{aligned}
$$

With these factors the equivalent nodal moments can be determined. The buoyancy force vector due to distributed pressure on a straight element is determined by assembling the nodal forces and moments of an element in one vector:

$$
\boldsymbol{F}_{1}^{\mathrm{b}}=\bar{q}_{\mathrm{b}}\left[\begin{array}{c}
b_{1} L \boldsymbol{e}_{\mathrm{n}} \\
b_{\mathrm{m} 1} L^{2}\left(\boldsymbol{e}_{1} \times \boldsymbol{e}_{\mathrm{n}}\right) \\
b_{2} L \boldsymbol{e}_{\mathrm{n}} \\
b_{\mathrm{m} 2} L^{2}\left(\boldsymbol{e}_{1} \times \boldsymbol{e}_{\mathrm{n}}\right)
\end{array}\right]
$$

Here $\boldsymbol{e}_{\mathrm{n}}$ is the direction of the equivalent forces and $\left(\boldsymbol{e}_{1} \times \boldsymbol{e}_{\mathrm{n}}\right)$ is the direction of the equivalent moments.

\section{Curvature effects}

Hydrostatic loads due to the curvature of the pipe are implemented as given by Yazdchi and Crisfield [66]. When the pipe is curved, the elements are connected at an angle, as can be seen in Figure 5.2. This angle is determined by the local element rotations. Due to the angle the element has an increased surface area at the outer curve in comparison with that of a straight pipe. At the inner curve the element has a decreased surface area. This is illustrated in Figure 5.4.

Forces due to curvature effects are determined by integrating the water pressure over the additional surface area at the outer curve. The reduction in surface area at the inner curve is equal in size to the increase in surface at the outer curve. 


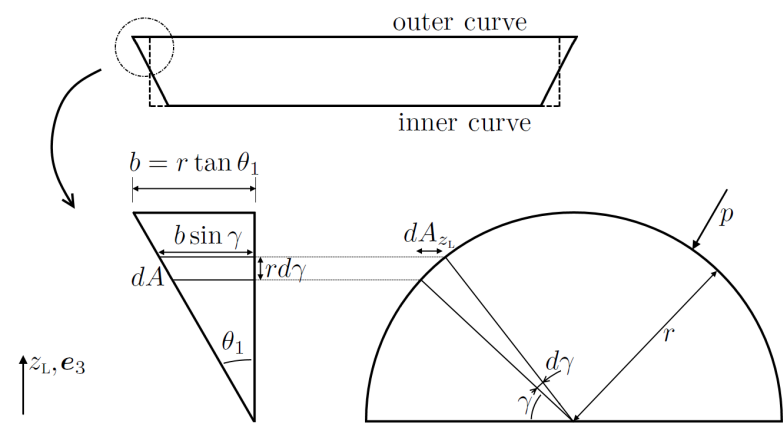

Figure 5.4: Increased surface area at the outer curve of the pipe and decreased surface area at the inner curve of the pipe.

The force due to this reduction is accounted for by multiplying the integral over the additional surface area by factor 2 .

In Figure 5.4 a side view and a front view of the additional surface are is given. In this derivation the pipe is bent about the $y_{\mathrm{L}}$-axis, such that the force is directed in $z_{\mathrm{L}}$ direction. At the first node the local rotation is $\theta_{1}$, as defined in Section 2.1. In the figure an area increment $d A$ is defined:

$$
d A=r^{2} \sin \gamma \tan \theta_{1} d \gamma
$$

From this area increment a projected area increment can be determined: $d A_{z_{\mathrm{L}}}=$ $\sin \gamma d A$. The force at the first node in $z_{\mathrm{L}}$-direction is determined by integrating the pressure over the projected area:

$$
F_{\text {curv }}=2 \int_{x_{\mathrm{L}}} \int_{0}^{\pi} p \sin \gamma d A=q_{\mathrm{b}} h_{1} \tan \theta_{1}
$$

The force in $y_{\mathrm{L}}$-direction and the forces at the second node can be determined similarly. Combining these forces yields the contribution of curvature effects to the buoyancy loads:

$$
\boldsymbol{F}_{2}^{\mathrm{b}}=q_{b}\left[\begin{array}{c}
h_{1} \tan \theta_{1} \boldsymbol{e}_{3}-h_{1} \tan \phi_{1} \boldsymbol{e}_{2} \\
\mathbf{0}_{3 \times 1} \\
-h_{2} \tan \theta_{2} \boldsymbol{e}_{3}+h_{2} \tan \phi_{2} \boldsymbol{e}_{2} \\
\mathbf{0}_{3 \times 1}
\end{array}\right]
$$

The forces due to curvature effects are dependent on $h_{1}$ and $h_{2}$, thus the buoyancy forces are dependent on the distance between the pipe and the surface of the water.

\section{Capped ends}

The end of a submerged pipe can be open or closed. When the pipe is closed with a cap, the water pressure acts on this cap. A buoyancy term for the capped end is 
also given by Yazdchi and Crisfield. It is derived by integrating the water pressure over the surface area of the capped end. The following equations show the capped end term on the first and second node, respectively:

$$
\boldsymbol{F}_{3}^{\mathrm{b}}=q_{b}\left[\begin{array}{c}
h_{1} \boldsymbol{e}_{1} \\
\mathbf{0}_{3 \times 1} \\
\mathbf{0}_{3 \times 1} \\
\mathbf{0}_{3 \times 1}
\end{array}\right] \quad \text { or } \quad \boldsymbol{F}_{3}^{\mathrm{b}}=q_{b}\left[\begin{array}{c}
\mathbf{0}_{3 \times 1} \\
\mathbf{0}_{3 \times 1} \\
-h_{2} \boldsymbol{e}_{1} \\
\mathbf{0}_{3 \times 1}
\end{array}\right]
$$

\section{Correction on cross-sectional area}

In the buoyancy calculation the elements are assumed straight. At the nodes the elements are connected at a certain angle, as illustrated in Figure 5.5. $\beta_{1}$ and $\beta_{2}$ indicate the local angles of rotation at the intersecting node. Yazdchi and Crisfield [66] assume that the cross-sectional area of both elements is equal at their common node, thus $\beta_{1}=\beta_{2}$. When, for example, two adjacent elements have different lengths, $\beta_{1} \neq \beta_{2}$. This results in an additional area perpendicular to $\boldsymbol{t}_{1}$, on which the water pressure acts. Therefore, a correctional force is derived based on the difference between the cross-sectional areas. The total cross-sectional
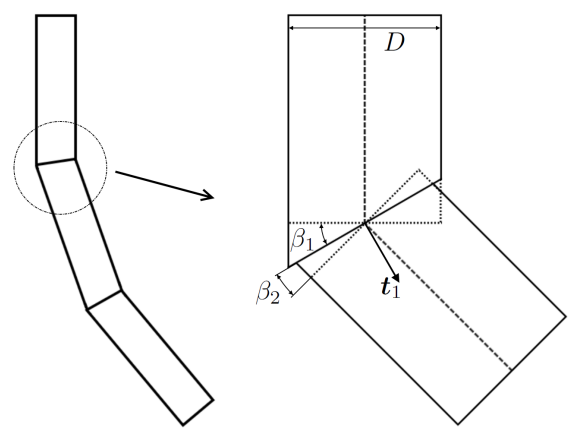

Figure 5.5: The connection of two buoyancy elements.

area when local rotation $\beta_{1}=0$ is the surface area of a circle. The total area when $\beta_{1} \neq 0$ is equal to:

$$
A=\frac{\pi D^{2}}{4 \cos \beta_{1}}
$$

When the local rotation of the adjacent element $\beta_{2}$ is unequal to the local rotation of the current element $\beta_{1}$, there is a difference in area equal to:

$$
\Delta A=\frac{\pi D^{2}}{4 \cos \beta_{1}}-\frac{\pi D^{2}}{4 \cos \beta_{2}}
$$

In the calculation of the forces of the current element, the local rotation of the adjacent element is unknown. Therefore, the difference with respect to $\beta_{2}=0$ is 
calculated.

$$
A=A_{\text {ext }}\left(\frac{1}{\cos \beta_{1}}-1\right)
$$

The force acting on this area is equal to the pressure multiplied by the area. To expand to three dimensions, $\cos \beta_{1}$ is substituted with $\boldsymbol{t}_{1}^{T} \boldsymbol{e}_{1}$. This results in the following force vector for one node:

$$
\boldsymbol{F}^{\text {node }}=q_{\mathrm{b}} h_{1}\left(\frac{1}{\boldsymbol{t}_{1}^{T} \boldsymbol{e}_{1}}-1\right) \boldsymbol{t}_{1}
$$

In context of an element vector $\boldsymbol{t}_{1}$ is renamed $\boldsymbol{u}_{1}$, as explained in Section 2.1. This results in the following force vector for the area mismatch of one element:

$$
\boldsymbol{F}_{4}^{\mathrm{b}}=q_{\mathrm{b}}\left[\begin{array}{c}
h_{1}\left(\frac{1}{\boldsymbol{t}_{1}^{T} \boldsymbol{e}_{1}}-1\right) \boldsymbol{t}_{1} \\
\mathbf{0}_{3 \times 1} \\
-h_{2}\left(\frac{1}{\boldsymbol{u}_{1}^{T} \boldsymbol{e}_{1}}-1\right) \boldsymbol{u}_{1} \\
\mathbf{0}_{3 \times 1}
\end{array}\right]
$$

The minus-one terms in this force vector could be removed, as they are cancelled out by the terms of the adjacent elements when constructing the system force vector. When it is left out, the capped end term needs to be removed to avoid calculating the capped end area twice. Here, it is chosen to keep the minus one term, in order to illustrate that the correctional force is zero for a straight pipe.

The importance of the new buoyancy term is demonstrated by an example of a curved pipe at a water depth of $2000 \mathrm{~m}$. Its left end is fixed and the right end is rotated to an angle of $\pi / 2$ by a prescribed rotation about the $y$-axis. Pipe and water properties are presented in Table 5.1. Three elements are used, two of which have length $L=25 \mathrm{~m}$ and one has length $L=50 \mathrm{~m}$. The node that connects two unequally sized elements has different local rotations for both elements, as can be seen in Figure 5.6.

The reaction forces at the fixed pipe end are shown in Table 5.2. The expected result from the summation of buoyancy forces in $x$-direction is zero. In $z$-direction, the expected result is the upthrust due to Archimedes' Law, which is $1 / 4 \pi D^{2} \rho g L=82.8 \mathrm{kN}$. From the results in the table, it can be concluded that the forces calculated without $\boldsymbol{F}_{4}^{\mathrm{b}}$ are incorrect. The results calculated including $\boldsymbol{F}_{4}^{\mathrm{b}}$, which is new in comparison with the buoyancy model of Yazdchi and Crisfield, are accurate. 


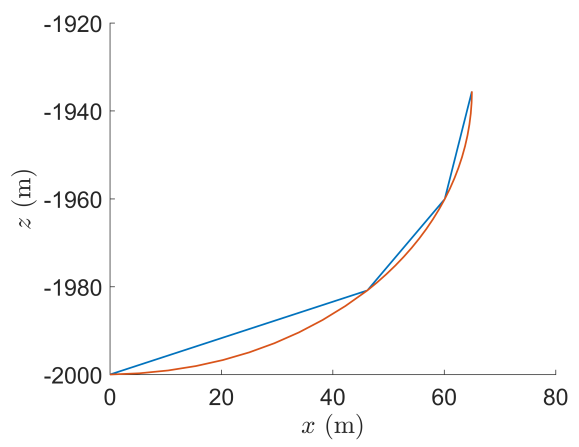

Table 5.1: Pipe and environmental properties of the vertical pipe example.

\begin{tabular}{lll}
\hline Length & $L$ & $100 \mathrm{~m}$ \\
Diameter & $D$ & $324 \mathrm{~mm}(13$ inch $)$ \\
Wall thickness & $t$ & $17.5 \mathrm{~mm}$ \\
Water density & $\rho_{\mathrm{w}}$ & $1025 \mathrm{~kg} / \mathrm{m}^{3}$ \\
\hline
\end{tabular}

Figure 5.6: Curved pipe at a water depth of $2000 \mathrm{~m}$.

Table 5.2: Reaction forces on the fixed pipe end.

\begin{tabular}{llll}
\hline & Theoretical & Without $\boldsymbol{F}_{4}^{\mathrm{b}}$ & With $\boldsymbol{F}_{4}^{\mathrm{b}}$ \\
\hline$F_{\mathrm{x}}$ & $0 \mathrm{kN}$ & $-63.6 \mathrm{kN}$ & $-1.9 \cdot 10^{-9} \mathrm{kN}$ \\
$F_{\mathrm{z}}$ & $82.8 \mathrm{kN}$ & $187.1 \mathrm{kN}$ & $82.8 \mathrm{kN}$ \\
\hline
\end{tabular}

\subsubsection{Comparison}

The Buoyancy model and the submerged weight model both approximate hydrostatic loads due to water pressure on the pipe's surface. The buoyancy model gives more accurate results because this model accounts for the actual load on the pipe. The submerged weight model uses an incorrect axial force and axial strain, which has to be corrected in a post-processing step.

When comparing computational costs, the submerged weight model is the least expensive. In this model the submerged weight load replaces the gravity load, thus no additional loads are applied. The buoyancy model applies additional loads, which require computation of additional stiffness matrix components. In this section an investigation is made of when the computationally inexpensive submerged weight model can be used and when the buoyancy model is required.

A difference between the results of the two models can be seen in an example of a pipe that is lowered vertically into the water. This happens for example during a free hanging start-up at the beginning of a pipe-laying operation. The buoyancy model and the submerged weight model apply different loads, as illustrated in Figure 5.7. Here, a schematic representation of two perfectly vertical submerged pipes is shown, each consisting of three elements. At the water surface all translations of the pipe's ends are constrained. At the first pipe loads resulting from the buoyancy model are shown, at the second pipe loads 
resulting from the submerged weight model are shown. These loads are defined in Equation (5.1). The buoyancy model applies a large force at the free end of the pipe due to a capped end. The submerged weight model reduces the gravity load equally over the length of the pipe and has no capped end force.

A difference in results from both models can also be seen in an example of a pipe that is lowered vertically in the water. Figure 5.8 shows a fully submerged vertical pipe. An additional horizontal force is applied to the free end of the pipe. The properties of the pipe and the water are shown in Table 5.3. The shapes of the pipe resulting from the buoyancy model and the submerged weight model are shown in Figure 5.9. It can be seen that the displacement of the free end is larger when using the buoyancy model. This is a result from the difference in applied loads, as illustrated in Figure 5.7.

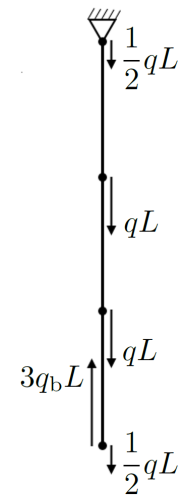

Buoyancy

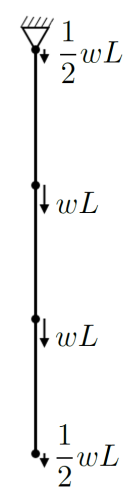

Submerged weight

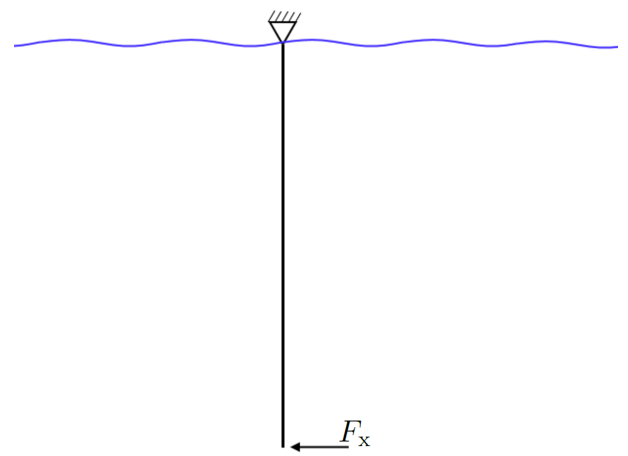

Figure 5.7: The loads on a vertical Figure 5.8: Submerged vertical pipe submerged pipe using two models. with a horizontal force at the free end.

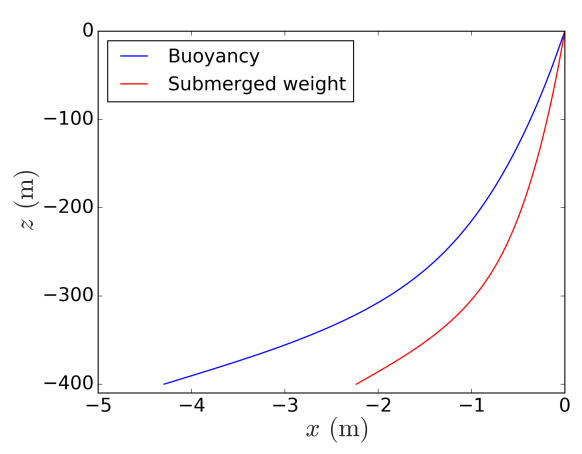

Figure 5.9: Resulting pipe shapes of submerged vertical pipe example.
Table 5.3: Pipe and environmental properties of the vertical pipe example.

\begin{tabular}{lll}
\hline Length & $L$ & $400 \mathrm{~m}$ \\
Diameter & $D$ & $457 \mathrm{~mm}(18$ inch $)$ \\
Wall Thickness & $t$ & $30.8 \mathrm{~mm}$ \\
Young's modulus & $E$ & $207 \mathrm{GPa}$ \\
Poisson's ratio & $\nu$ & 0.3 \\
Density & $\rho$ & $7850 \mathrm{~kg} / \mathrm{m}^{3}$ \\
Water density & $\rho_{\mathrm{w}}$ & $1025 \mathrm{~kg} / \mathrm{m}^{3}$
\end{tabular}

Horizontal force $F_{\mathrm{x}} \quad 1 \mathrm{kN}$ 
The buoyancy model and the submerged weight model are also compared in an S-lay example with a linear-elastic material model. Tables 5.4 and 5.5 show the properties of the pipe, the input variables and the environmental properties used in this example. Figure 5.10a compares the shapes of the pipe when using the buoyancy model and the submerged weight model. It can be seen that the results are in good agreement with each other. The computational time of this S-lay example with the submerged weight model is approximately $17 \%$ shorter than the computational time with the buoyancy model.

Axial strains resulting from the S-lay example are compared in Figure 5.10b. Here, three results are compared: from the buoyancy model, from the submerged weight model and from the submerged weight model without the correction on the axial force and strain. The post-processing step has no influence on the shape of the pipe. It can be seen that when the buoyancy model is applied without the correction in the post-processing step the axial strain is always positive. The results of the buoyancy model and the submerged weight model with the post-processing correction are negative near the seabed and are in good agreement with each other.

Table 5.4: Properties of the pipe in the S-lay example.

Table 5.5: Input variables and environmental properties of the S-lay

\begin{tabular}{ll}
\hline Length & $2200 \mathrm{~m}$ \\
Diameter & $273 \mathrm{~mm}$ (10.75 inch) \\
Wall Thickness & $20.6 \mathrm{~mm}$ \\
Young's modulus & $207 \mathrm{GPa}$ \\
Poisson's ratio & 0.3 \\
Density & $7850 \mathrm{~kg} / \mathrm{m}^{3}$ \\
\hline
\end{tabular}
example.

\begin{tabular}{ll}
\hline Bottom tension & $115 \mathrm{kN}$ \\
Water depth & $1710 \mathrm{~m}$ \\
Water density & $1025 \mathrm{~kg} / \mathrm{m}^{3}$ \\
Roller-box penalty & $70000 \mathrm{kN} / \mathrm{m}$ \\
Seabed penalty & $100 \mathrm{kN} / \mathrm{m}$ \\
\hline
\end{tabular}

It is recommended to use the submerged weight model in J-lay and S-lay simulation with linear-elastic material models. In more detailed simulations it is recommended to use the buoyancy model. An example of such an analysis is when plastic material deformation occurs in a submerged pipe segment. This will be investigated in Chapter 6. Another example is the detailed analysis of the torsional rotation of a pipe during laying, such as the study of O'Grady and Harte [45]. The example from Figure 5.9 shows that simulating a submerged pipe with a free end, such as a free hanging start-up in a pipe-lay project, also requires a detailed buoyancy model.

\subsection{Hydrodynamic loads}

Submerged pipes are subject to hydrodynamic loads, which are a result of the interaction between pipe and water. When the pipe moves through water, it is 


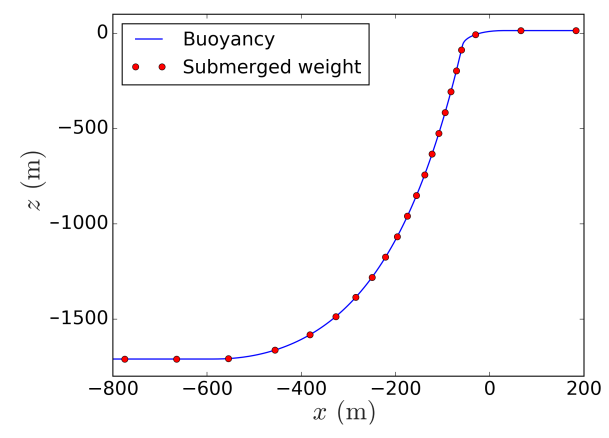

(a) Shape of the pipe.

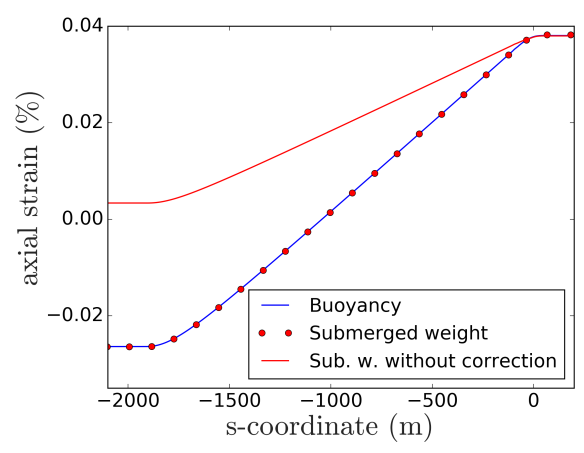

(b) Axial strain in the pipe.

Figure 5.10: Buoyancy and submerged weight compared in an S-lay example using linear-elastic material.

subject to fluid resistance. When the water moves and accelerates due to surface waves, it exerts a load on the pipe. In the equation of motion hydrodynamic loads are part of the external force vector.

In models of offshore structures hydrodynamic loads on cylindrical objects due to moving water are often estimated by Morison's equation [39], which can be modified for moving cylindrical objects [22]. The flow of the water is determined by the Airy wave theory, as explained in Section 5.3. In Morison's equation it is assumed that the velocities and accelerations of the water are not influenced by the presence of the pipe, thus that the pipe diameter is small in relation to the wavelength of the surface wave. Furthermore, loads resulting from flow parallel to the pipe are disregarded. Morison's equation is often used because it is easy to use and gives sufficiently accurate load predictions.

In the pipe-lay model Morison's equation is used to calculate a load is that is uniformly distributed over the length of the pipe. In the finite element implementation this distributed load is acting on submerged elements. The nodal equivalent forces are determined by assuming that the load is equally distributed over both nodes:

$$
\boldsymbol{F}^{\text {Morison }}=\frac{1}{2} L_{0} \boldsymbol{q}
$$

Modified for a moving pipe in moving water Morison's equation is given by [22]:

$$
\boldsymbol{q}^{\text {Morison }}=\frac{1}{2} \rho_{\mathrm{w}} C_{\mathrm{d}} D \dot{\boldsymbol{d}}_{\mathrm{rn}}\left\|\dot{\boldsymbol{d}}_{\mathrm{rn}}\right\|+\rho_{\mathrm{w}} C_{\mathrm{a}} \frac{\pi}{4} D^{2} \ddot{\boldsymbol{d}}_{\mathrm{rn}}+\rho_{\mathrm{w}} \frac{\pi}{4} D^{2} \ddot{\boldsymbol{d}}_{\mathrm{wn}}
$$

Here, $\rho_{\mathrm{w}}$ is the density of the water and $D$ is the pipe diameter. $C_{\mathrm{d}}$ and $C_{\mathrm{a}}$ are the drag and added mass coefficients, which can be determined empirically. $\dot{\boldsymbol{d}}_{\mathrm{rn}}$ and $\ddot{\boldsymbol{d}}_{\mathrm{rn}}$ are the relative velocity and relative acceleration of the water in normal 
direction to the pipe. $\ddot{\boldsymbol{d}}_{\mathrm{wn}}$ is the acceleration of the water in normal direction to the pipe.

The first term of Equation (5.22) is a drag force caused by viscous friction between the pipe and the water. The second term is an inertia term, often referred to as added mass. It results from the inertia of the water around the pipe, which is accelerated due to accelerations of the pipe. The third term, which is named the Froude-Krylov force, is the effect of the pressure gradient which causes acceleration of the water. Together, these three terms form Morison's equation.

The relative velocity and relative acceleration of the water are calculated with respect to those of the pipe:

$$
\begin{aligned}
& \dot{\boldsymbol{d}}_{\mathrm{r}}=\dot{\boldsymbol{d}}_{\mathrm{w}}-\dot{\boldsymbol{d}} \\
& \ddot{\boldsymbol{d}}_{\mathrm{r}}=\ddot{\boldsymbol{d}}_{\mathrm{w}}-\ddot{\boldsymbol{d}}
\end{aligned}
$$

The relative velocity and relative acceleration of the water in the direction normal to the pipe are calculated by subtracting their tangential parts:

$$
\begin{aligned}
& \dot{\boldsymbol{d}}_{\mathrm{rn}}=\left(\mathbf{I}-\boldsymbol{e}_{1} \boldsymbol{e}_{1}^{\mathrm{T}}\right) \dot{\boldsymbol{d}}_{\mathrm{r}} \\
& \ddot{\boldsymbol{d}}_{\mathrm{rn}}=\left(\mathbf{I}-\boldsymbol{e}_{1} \boldsymbol{e}_{1}^{\mathrm{T}}\right) \ddot{\boldsymbol{d}}_{\mathrm{r}}
\end{aligned}
$$

The velocity and acceleration of the pipe are determined by the time integration method, as shown in Equation (3.3). The velocity and acceleration of the water will be derived in Section 5.3.

The hydrodynamic forces resulting from Morison's equation are external forces. All external forces that depend on the nodal displacements or rotations lead to a contribution to the stiffness matrix when properly linearised. The contribution to the stiffness matrix of Morison's equation consists of three parts: stiffness due to the drag force, added mass and pressure gradient. The derivations of these three parts can be found in Appendix A.

\subsection{Waves and vessel response}

At the surface of the water, waves are created by an interaction between water and wind. When extreme weather conditions are expected, the pipe-laying vessel abandons the pipe by lowering the unfinished pipe onto the seabed. When the weather conditions have improved, the pipe-laying vessel recovers the abandoned pipe and continues pipe-laying. Extreme sea states, such as huge waves during a storm, are not required in the model. It is assumed that the amplitude of the surface wave is small in relation to the wavelength and water depth. Surface waves and the corresponding velocities and accelerations of the water beneath the water surface are modelled with linear theory. 
A regular wave such as a single cosine function is insufficient to model surface waves. They should be modelled as a superposition of regular waves. Beneath the water surface the Airy wave theory [19] is used to describe motions of the seawater. In this theory a constant water depth and irrotational flow is assumed. The waves have a constant frequency and their motions are described in two dimensions: in horizontal and vertical direction.

The motions of the vessel's centre of gravity are determined as a linear response to the surface waves using a transfer function [22]. It is assumed that the combination of the vessel and the stinger is rigid, such that the motions of the roller-boxes follow directly from the motions of the centre of gravity.

The response of the vessel's centre of gravity is determined in the vessel's coordinate system, which has its origin in the centre of gravity and is stationary. The sailing direction of the vessel during pipe-laying is in positive $x_{\mathrm{V}}$-direction, thus the stinger points in negative $x_{\mathrm{V}}$-direction. In this coordinate system six degrees of freedom are defined. Heave, sway and surge are translational degrees of freedom in $x_{\mathrm{V}}, y_{\mathrm{V}}$ and $z_{\mathrm{V}}$-directions and roll, pitch and yaw are rotational degrees of freedom about the $x_{\mathrm{V}}, y_{\mathrm{V}}$ and $z_{\mathrm{V}}$-axes. The vessel's coordinate system and its degrees of freedom are illustrated in Figure 5.11.

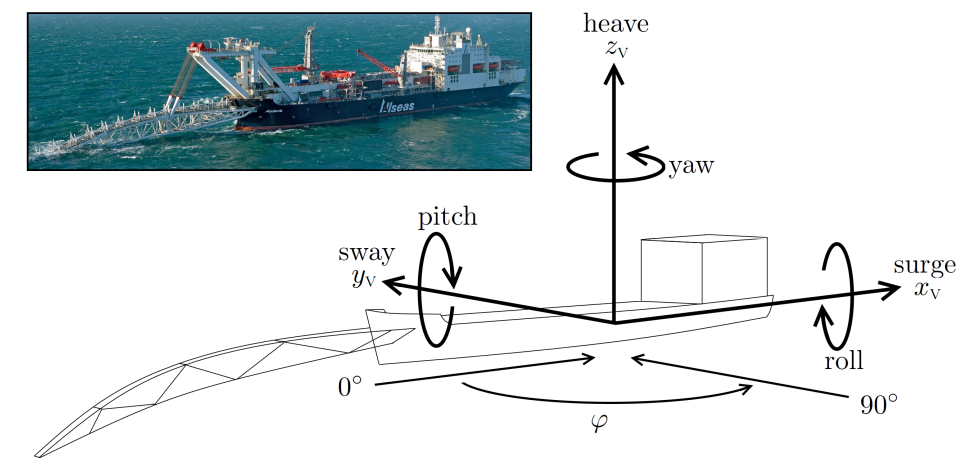

Figure 5.11: Pipe-laying vessel's coordinate system and degrees of freedom.

The vessel's motions are dependent on encountering waves on the water surface. The direction of the waves on this surface is determined by an encountering wave angle $\varphi$, which is the counter-clockwise angle between the vessel's $x_{\mathrm{V}}$-axis and the encountering wave direction. The encountering wave angle is zero when the wave is travelling in the vessel's $x_{\mathrm{V}}$-direction, as indicated in Figure 5.11.

A single regular wave is described in Section 5.3.1. A regular wave is not a good representation of a random sea state; a superposition of regular waves can be a good representation. In Section 5.3.2, generic waves are modelled by superposition of regular waves. Section 5.3.3 presents the response of the vessel's centre of gravity to regular and generic waves. 


\subsubsection{Regular wave}

A regular wave $\eta$ as a function of time and position can be parametrised with height $h$, frequency $\omega$ and wavenumber $k$ :

$$
\eta=\frac{h}{2} \cos (\omega t-k \bar{x})
$$

Position $\bar{x}$ is chosen relative to the centre of gravity of the vessel in direction of the wave, as indicated in Figure 5.12:

$$
\bar{x}=x_{\mathrm{V}} \cos \varphi+y_{\mathrm{V}} \sin \varphi
$$

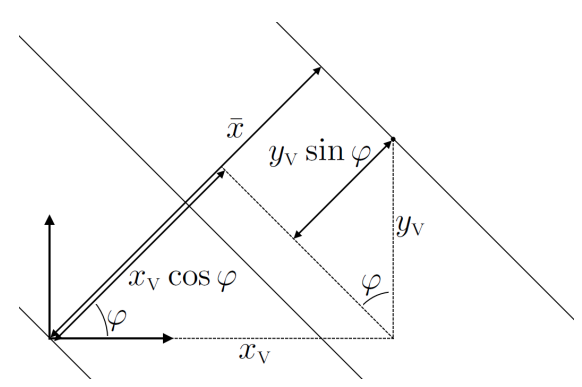

Figure 5.12: Encountering wave relative to the vessel's coordinate system, the contour lines indicate wave tops.

In the Airy wave theory a velocity potential is defined. The velocities of the water can be determined as spatial derivatives of the velocity potential. The Airy wave theory can be simplified when the water is deep. This simplification is valid when the water depth is greater than half the wavelength [19,22]. With this simplification, the velocity potential associated with Equation (5.25) is:

$$
\phi=-\frac{h g}{2 \omega} e^{k z} \sin (\omega t-k \bar{x})
$$

Here $g$ is the standard acceleration due to gravity and $z$ is a coordinate in the global coordinate system. The global coordinate system is chosen because $z=0$ at the water surface, but the vessel's coordinate $z_{\mathrm{V}}$ is not necessarily zero on the water surface.

The velocities of the water can be derived from the velocity potential, by taking the derivative with respect to the $x_{\mathrm{V}}, y_{\mathrm{v}}$ and $z$-directions. The accelerations of the water can be determined by taking the derivative of the velocities with respect to time.

$$
\dot{\boldsymbol{d}}_{\mathrm{w}}=\left[\begin{array}{c}
\cos \varphi \frac{h}{2} \omega e^{k z} \cos (\omega t-k \bar{x}) \\
\sin \varphi \frac{h}{2} \omega e^{k z} \cos (\omega t-k \bar{x}) \\
-\frac{h}{2} \omega e^{k z} \sin (\omega t-k \bar{x})
\end{array}\right] \quad \ddot{\boldsymbol{d}}_{\mathrm{w}}=\left[\begin{array}{c}
-\cos \varphi \frac{h}{2} \omega^{2} e^{k z} \sin (\omega t-k \bar{x}) \\
-\sin \varphi \frac{h}{2} \omega^{2} e^{k z} \sin (\omega t-k \bar{x}) \\
-\frac{h}{2} \omega^{2} e^{k z} \cos (\omega t-k \bar{x})
\end{array}\right]
$$




\subsubsection{Generic wave}

In reality, waves at the surface of the sea are not perfect regular waves. At sea, waves are created by the interaction between water and wind. A high wind velocity over a long period creates high waves. In ocean engineering these generic waves can be modelled by a collection of regular waves of different frequencies.

In this collection each regular wave has a particular amplitude and frequency. To determine the amplitude of a regular wave at a certain frequency, a wave spectrum is used. This wave spectrum gives the distribution of a 'wave spectral density' as a function of the wave frequency and has unit $\mathrm{m}^{2} \mathrm{~s}$. All the regular waves in the collection are given a certain frequency, the wave spectrum determines the corresponding amplitude.

Different wave spectra can be found in literature, such as the JONSWAP (JOint North Sea WAve Project) spectrum [26], the Pierson-Moskowitz spectrum [49] and the Bretschneider spectrum [9]. All these spectra assume that the wind has a constant velocity in a constant direction over a long time period, representing fully developed waves at sea.

A Pierson-Moskowitz spectrum has been implemented in the pipe-lay model. It is based on a spectrum density $S$ [3]:

$$
\begin{aligned}
S(\omega)=\frac{B}{\omega^{5}} e^{\frac{-C}{\omega^{4}}} & =4 \pi^{3} \frac{H_{\mathrm{s}}^{2}}{T_{\mathrm{z}}^{4}} \\
C & =\frac{16 \pi^{3}}{T_{\mathrm{z}}^{4}}
\end{aligned}
$$

Here, $H_{\mathrm{s}}$ is the significant wave height and $T_{\mathrm{z}}$ is the mean zero up-crossing period, which is the average period between crossing the average water surface in an upward direction. An example of a Pierson-Moskowitz spectrum is given in Figure 5.13 , which is determined with significant wave height $H_{\mathrm{s}}=3 \mathrm{~m}$ and mean zero up-crossing period $T_{\mathrm{z}}=7 \mathrm{~s}$.

The wave surface profile is determined by superposition of regular waves within a frequency range. The wave height of each of these waves is determined from the continuous wave spectrum density at discrete frequencies $\omega_{i}$, which are separated by frequency step $\Delta \omega$.

$$
h_{i}=2 \sqrt{2 S(\omega) \Delta \omega}
$$

In the summation of regular waves the phase angle is randomised by parameter $\epsilon_{i}$, which have values between 0 and $2 \pi$.

$$
\eta(\bar{x}, t)=\sum \frac{h_{i}}{2} \cos \left(\omega_{i} t-k_{i} \bar{x}+\epsilon_{i}\right)
$$




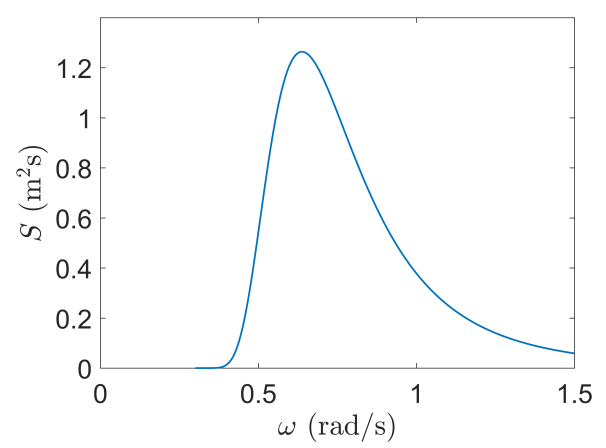

Figure 5.13: Pierson-Moskowitz wave spectrum with $H_{\mathrm{s}}=3 \mathrm{~m}$ and $T_{\mathrm{z}}=7 \mathrm{~s}$.

\subsubsection{Vessel response}

The vessel's motions are defined as a linear response to the wave surface profile at the centre of gravity [22]. This is done by using response amplitude operators, or RAOs, which are linear transfer functions that map the wave motions to the vessel's motions. At a certain frequency each of the vessel's degrees of freedom has two RAO parameters: $\Lambda$ for the amplitude and $\zeta$ for the phase. For a regular wave the response $\xi$ is calculated as:

$$
\xi(t)=\Lambda \frac{h}{2} \cos (\omega t+\zeta)
$$

For a generic wave the contribution of each frequency is summed:

$$
\xi(t)=\sum \Lambda_{i} \frac{h_{i}}{2} \cos \left(\omega_{i} t+\epsilon_{i}+\zeta_{i}\right)
$$

The difference between the vessel's response to a regular wave and a generic wave is shown in Figure 5.14. Figure 5.14a shows the displacements of the centre of gravity of a pipe-laying vessel in global $x, y$ and $z$-direction, in response to a regular wave with $h=3 \mathrm{~m}$ and $T=11 \mathrm{~s}$. Figure $5.14 \mathrm{~b}$ shows the displacements of this centre of gravity in response to a generic wave defined by the spectrum shown in Figure 5.13.

\subsection{Modelling considerations}

In the previous sections of this chapter equations and algorithms for external loads and prescribed displacements are presented. Combined with the basis for a pipe-lay model from the previous chapters, a pipe-lay analysis with a linear elastic material model can be performed. This section gives two considerations 


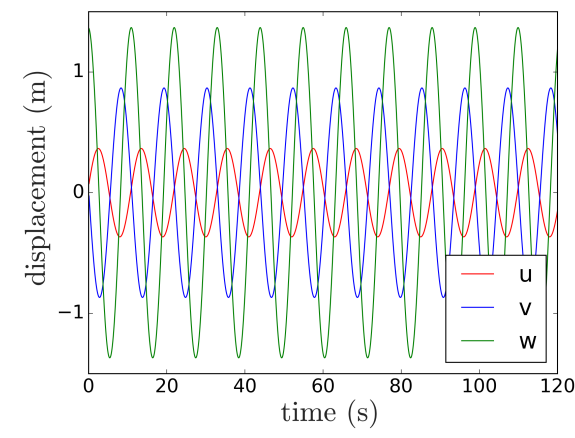

(a) Regular wave with $h=3 \mathrm{~m}$ and $T=$ $11 \mathrm{~s}$.

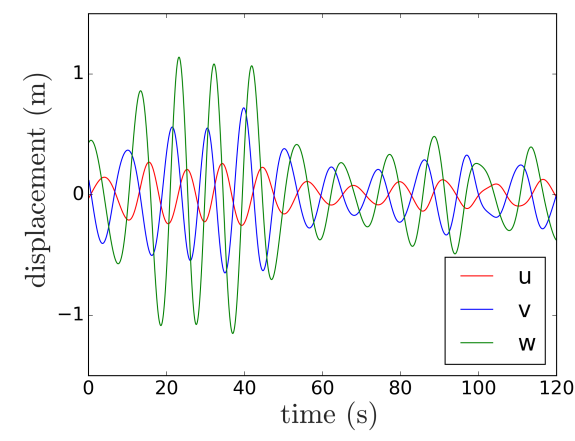

(b) Generic wave with $H_{s}=3 \mathrm{~m}$ and $T_{z}=$ $7 \mathrm{~s}$.

Figure 5.14: Response of the centre of gravity of a pipe-laying vessel to a regular and a generic wave.

on this pipe-lay model. The first consideration on the pipe-lay model is about the maximum time step size, which is determined from the frequencies of the vessel's motions. The second consideration on the pipe-lay model is about axial compression in submerged elements, which can give an issue with convergence. This issue is addressed and a solution is presented.

\subsubsection{Maximum time step size}

Stability of the HHT- $\alpha$ method for numerical time integration does not restrict the time step size. Convergence of non-linear sub-models of the pipe-lay model, such as contact or plasticity, can limit the time step size. In all presented submodels attention is given to preventing limitations on time step sizes, such as the implementations of the contact model in Section 4.4 and the plasticity model in Section 6.2. The remaining limitation on the time step size is the accuracy of the results from the pipe-lay model. This limitation is investigated in the present section.

The maximum time step size is related to the highest relevant frequency in the model. Assuming that the resulting displacements and rotations of the pipe do not contain frequencies of interest that are higher than the highest frequency in the motion of the pipe-lay vessel, the highest relevant frequency can be determined from the wave spectrum and the RAOs.

The response of a pipe-laying vessel to the wave spectrum shown in Figure 5.13 can be described by multiplying the amplitude RAO $\Lambda$ by the wave amplitude $h / 2$ for each degree of freedom. The result is shown in Figure 5.15, where it can be seen that the vessel responses are very small when $\omega>1 \mathrm{rad} / \mathrm{s}$. 


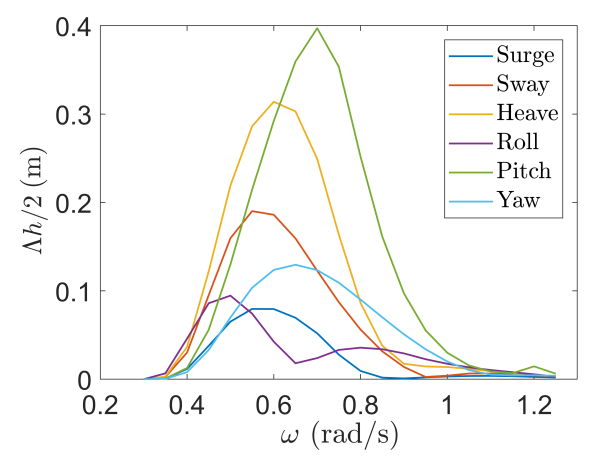

Figure 5.15: Response of a pipe-laying vessel to wave frequencies for all its degrees of freedom.

The equation of motion is evaluated at the end of each time increment, thus force balance is ensured at each discrete point in time. The numerical time integration scheme can predict an incorrect amplitude decay and period elongation [29]. It is assumed that these numerical errors are not governing in the selection of the time step size, because the amplitude decay and the period elongation are continuously corrected by the excitation of the pipe by the vessel. Additionally, hydrodynamic damping is much stronger than numerical damping.

For determining the maximum strains during pipe-laying, the maximum displacements of the pipe are relevant. When a time step is too large, this maximum might be too far away from a discrete time to be correctly modelled. The time step must be sufficiently small to predict the maximum displacements of the pipe.

From Figure 5.15 a maximum frequency of $\omega=1 \mathrm{rad} / \mathrm{s}$ was obtained. This maximum frequency is valid for this particular pipe-laying vessel with this particular wave spectrum. It is assessed whether or not a time step of $\Delta t=1 \mathrm{~s}$, which corresponds to approximately six time increments per wave period, is sufficiently small.

The error with this time step can be quantified by the difference between the peak value of a sine function and the value of this function at a discrete time. The error is zero when the discrete time results in the peak of the function. The error is maximal when the peak of the sine function is in the middle of a time interval. The maximum error can be quantified with:

$$
\epsilon_{\text {sine }}=1-\cos \left(\omega \frac{1}{2} \Delta t\right)
$$

Using a time step of $\Delta t=1 \mathrm{~s}$ and a frequency of $\omega=1 \mathrm{rad} / \mathrm{s}$ results in a maximum error of approximately $12 \%$. This might seem high, but it is applicable only to the contribution of the wave at maximum frequency. The contributions of all other 
frequencies, which have larger amplitudes, have a smaller error. In Figure 5.15 it can be seen that the maximum motion of the vessel occurs when $\omega=0.7 \mathrm{rad} / \mathrm{s}$. At this frequency the maximum error is approximately $6 \%$.

The maximum error of each frequency can be quantified by scaling the error $\epsilon_{\text {sine }}$ with the corresponding amplitude. This results in an absolute error measure; a relative maximum error is obtained by scaling the error with the maximum amplitude in the frequency range:

$$
\epsilon_{\max }=\frac{\Lambda S}{\max (\Lambda S)} \epsilon_{\text {sine }}
$$

For each degree of freedom of the vessel this maximum relative error is shown in Figure 5.16. The actual error is between zero and the maximum relative error, which is sufficiency small.

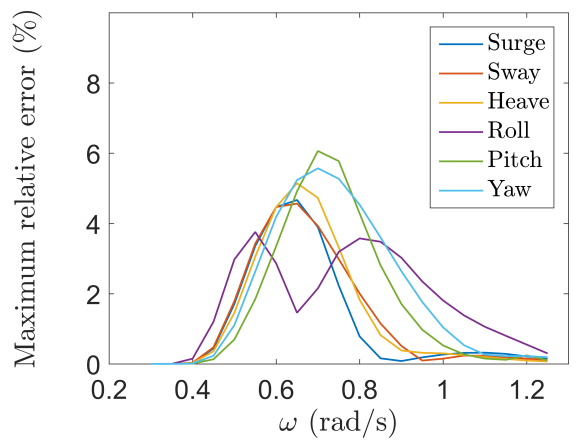

Figure 5.16: The maximum error at each frequency for all of a pipe-laying vessel's degrees of freedom.

\subsubsection{Axial compression in submerged elements}

During pipe-laying axial tension is applied to the pipe by the tensioner on board the S-lay vessel. In deep water the pipe can be in compression due to the hydrostatic forces acting on the pipe, as shown in Figure 5.10b. In numerical examples it is observed that when a negative axial strain occurs local rotations can be predicted incorrectly. In this section the cause of these incorrect predictions is explained and an approach to getting correct results is presented.

In Section 2.5 it was shown that co-rotational elements can predict incorrect rotations when the pipe's bending stiffness is much smaller than its axial stiffness. This prediction is corrected by including second-order terms for the axial strain due to the curvature of an element. When the axial force is positive, these second-order terms increase the bending stiffness, preventing the incorrect rotations. When the axial force is negative, the element's bending stiffness 
decreases, potentially making the incorrect predictions worse instead of correcting them.

This issue is illustrated with a J-lay example. In this example the part of the pipe near the seabed will be under axial compression. For this part a transition from elements of length $50 \mathrm{~m}$ to elements of length $25 \mathrm{~m}$ is modelled. Properties of this example are given in Table 5.6. Figure 5.17 shows the shape of the pipe resulting from this J-lay example, the element sizes are indicated in the figure.

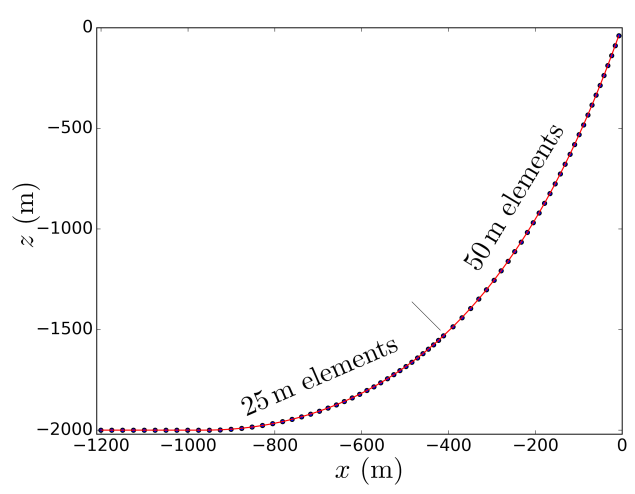

Table 5.6: Properties of the J-lay example.

\begin{tabular}{ll}
\hline Length & $2600 \mathrm{~m}$ \\
Diameter & $356 \mathrm{~mm}$ (14 inch) \\
Wall thickness & $29.3 \mathrm{~mm}$ \\
Bottom tension & $500 \mathrm{kN}$ \\
Water depth & $2000 \mathrm{~m}$ \\
\hline
\end{tabular}

Figure 5.17: Shape of the pipe resulting from the J-lay example.

Figure 5.18 shows the bending strain and axial strain resulting from this example as a function of an s-coordinate, which is a coordinate along the length of the pipe. In Figure 5.18a the results are determined without second-order terms for the element forces and moments, in Figure 5.18b with these second-order terms. When the second-order terms are disregarded, there is a peak in bending strain at the transition from the element with length $25 \mathrm{~m}$ to the element with length $50 \mathrm{~m}$. There is also a peak in bending strain at the pipe end that is connected to the laying vessel. When the second-order terms are included, the peak in strain at the pipe end, where the axial force is positive, disappears. Near the seabed, where the axial force is negative, new peaks arise and the peak in bending strain at the element size transition becomes larger.

When a beam element is in compression its bending stiffness is reduced. A long slender beam with a reduced bending stiffness cannot be loaded with a moment, analogical to a cable or wire. In the pipe-lay model distributed loads are modelled by equivalent nodal forces and moments. When a cable is loaded with a distributed load, it would not be modelled with an equivalent moment because a moment cannot be applied to a cable. Therefore, the distributed loads must be modelled with equivalent forces only. This can be done by setting all equivalent moments to zero where the axial force is negative. 


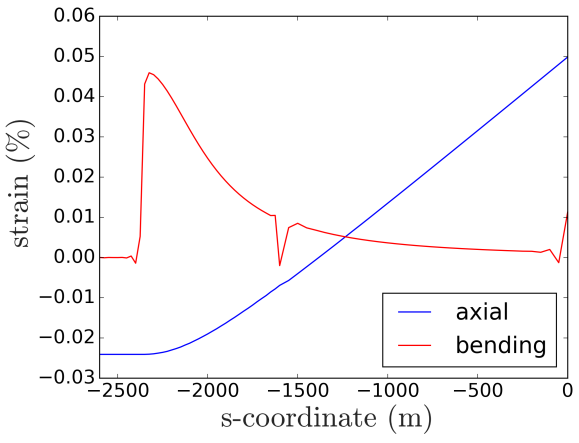

(a) Without second-order terms.

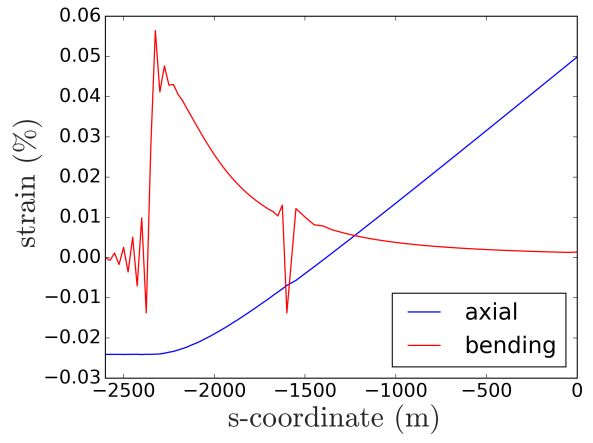

(b) Including second-order terms.

Figure 5.18: Axial and bending strains over the length of the pipe when considering the pipe as a beam.

The second-order terms in the calculation of the element moments cause peaks in the bending strain near and on the seabed. At the seabed contact forces are applied on the nodes. Between the nodes the pipe can penetrate the surface without resistance. In combination with the low bending stiffness, this results in incorrect large rotations, as in Figure 2.11a. This issue can be solved by setting the second-order terms in the calculation of the element moments to zero when the axial force is negative.

When the element is in compression, these second-order terms model buckling effects. By disregarding these terms, buckling within an element is suppressed. Due to the co-rotational approach, buckling is still predicted by the model when more than one element is involved. Disregarding the second-order terms removes the incorrect strain peaks and does not discard the possibility of buckling in the model.

The approach to preventing incorrect predictions consists of two parts. The first part sets all equivalent nodal moments to zero when the axial force is negative. The second part sets the second-order terms in the calculation of the element's bending moments to zero when the axial force is negative. Figure 5.19 shows the resulting bending strain and axial strain of the J-lay example when using this approach. It can be seen that the artificial peaks in bending strain have disappeared.

\subsection{Closure}

In this chapter the static and dynamic basis for the pipe-lay model from the previous chapters was expanded with loads and prescribed displacement to create the pipe-lay model. With this model pipe-lay analyses with a linear elastic material model can be performed. 


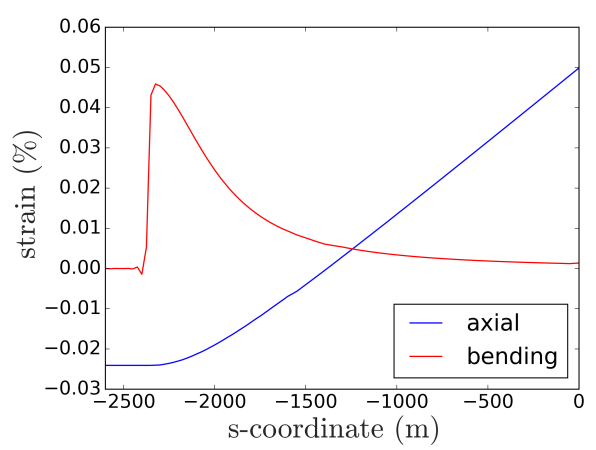

Figure 5.19: Strains over the pipe length with the approach to preventing incorrect predictions.

Buoyancy loads are commonly modelled by Archimedes' law, which requires a post-processing step in which the axial tension and strain are corrected. A method to describe buoyancy loads without this post-processing step was presented. Modelling buoyancy loads with Archimedes' law is insufficient when effects are involved that depend on the axial force or axial strain, such as plastic material behaviour and simulations with a free pipe end.

Hydrodynamic loads are modelled by Morison's equation, which consists of three parts: loads due to drag, due to added mass and due to a pressure gradient. The hydrodynamic loads result in damping.

Displacements of the roller-boxes on the stinger and the vessel are determined by surface waves and the vessel's response. Generic surface waves are determined from a superposition of regular waves. A wave spectrum determines the contribution to the amplitude of each frequency within a range. The motion of six degrees of freedom of the vessel's centre of gravity is linearly related to the motion of the wave. These responses are determined using RAOs.

At the end of this chapter two considerations of the developed pipe-lay model are given. The first consideration on the pipe-lay model concerns the maximum allowed time step size. This time step is not dependent on stability or convergence, but on the accuracy of the results. It is assumed that the highest frequency of the vessel's motion determines the highest frequency of the pipe's response, from which a maximum time step can be determined. The second consideration on the pipe-lay model concerns axial compression in submerged pipes in combination with second-order terms in the calculation of the element's bending moments. This combination can result in incorrect predictions of local rotations. An approach to getting correct predictions was presented. 


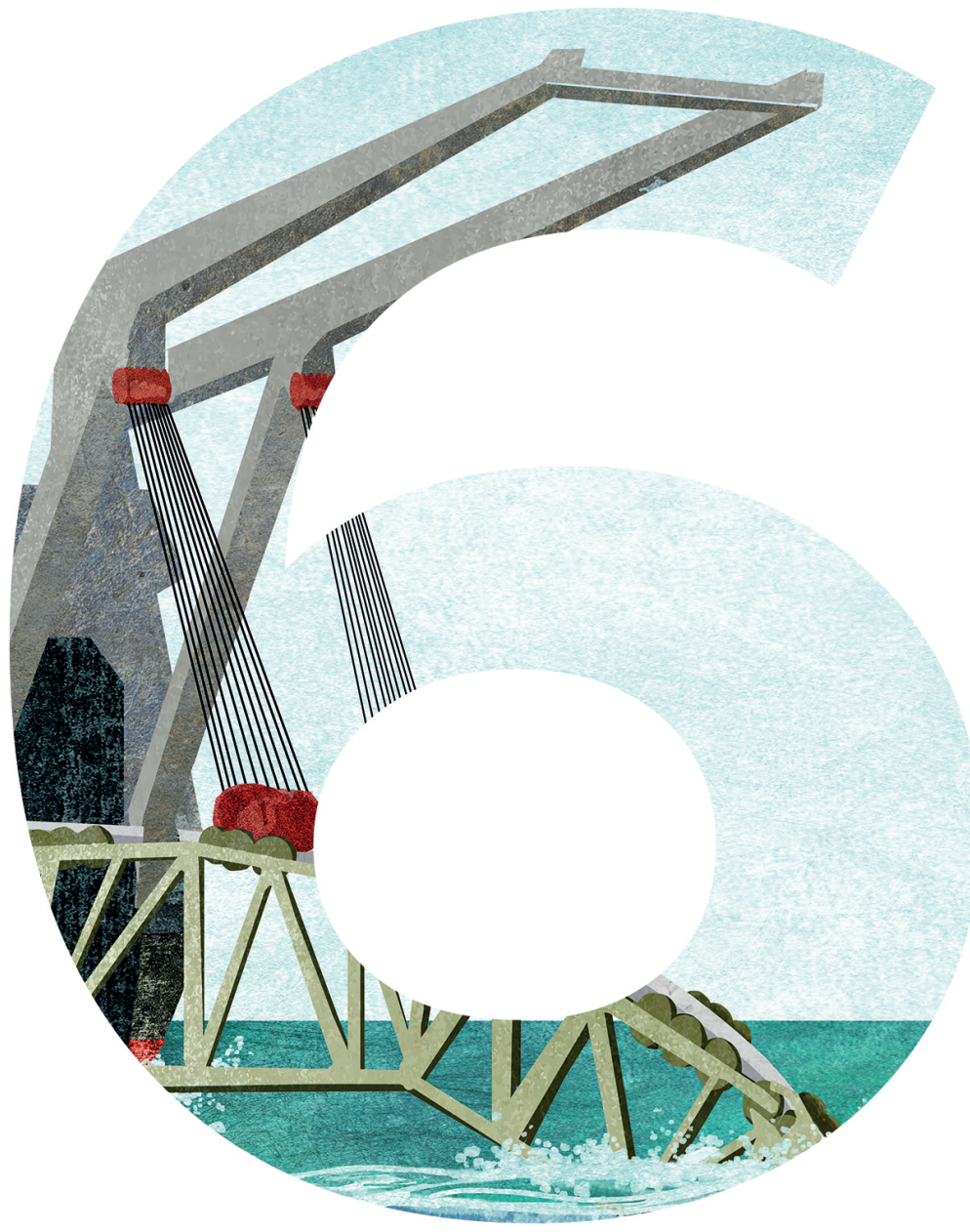




\section{6}

\section{Plasticity}

A pipe that is subject to a bending moment, normal force, torsion and pressure can deform plastically. When a pipe is installed with the S-lay method, the highest risk of plastic deformation is in the pipe section on the stinger. Especially when a stinger with a small radius is used in combination with a large diameter pipe. A stinger with a small radius is used in deep water, such that the pipe can leave the stinger almost vertically.

In this chapter a numerical approach to modelling plasticity in co-rotational pipe elements is presented. The resulting algorithm is implemented in the pipe-lay model presented in the preceding chapters.

In Section 6.1 a dedicated return mapping algorithm for elasto-plastic material deformation of thin-walled pipes is presented. In the derivation of the algorithm Von Mises plasticity and isotropic hardening is assumed. This section is based on De Vries et al. ${ }^{1}$

In Section 6.2 a numerical approach to applying the return mapping procedure in co-rotational pipe elements is presented. In each element stresses are evaluated at discrete integration points. These stresses are integrated over the volume of the pipe to obtain the internal element forces and moments.

\subsection{Dedicated algorithm for plasticity in pipes}

The radial return mapping algorithm is an efficient method for numerical modelling of elasto-plastic deformations. It was first described by Wilkins [62], restricted to a perfect plastic material model. Simo and Taylor [54] extended this algorithm with nonlinear hardening. Radial return mapping consists of two steps: an elastic

\footnotetext{
${ }^{1}$ F.H. de Vries, H.J.M. Geijselaers and A.H. van den Boogaard. Return mapping algorithm for elasto-plastic deformation of thin-walled pipes, Submitted, 2020
} 
predictor step, which can result in stresses outside the yield surface, and a plastic corrector step, to bring the stresses back on the yield surface. The term radial follows from the direction of the plastic corrector step, which is normal to the yield surface. When a von Mises yield surface is used, the direction of the plastic corrector step is radial.

Stress states in thin-walled structures are characterised by plane stress. Then, the return mapping procedure becomes more complicated because the direction of the plastic return mapping is no longer radial. In commercial simulation software, the plane stress condition is often enforced by imposing zero normal stress in the yield condition [41]. Without a dedicated 'local' plane stress algorithm this results in an extra 'global' iterative loop over the entire return mapping procedure, which is effective but time consuming.

Simo and Taylor [55] derived a plane stress return mapping algorithm in a plane stress subspace, which avoids this additional iteration loop. In the corrector step of this algorithm the stress state is mapped back on the yield surface by a consistency condition, which is a scalar nonlinear equation that is solved iteratively.

De Borst [16] introduced an alternative procedure for elasto-plastic plane stress plate and shell elements. In this procedure the stresses do not fulfil the plane stress condition exactly but converge to it during the equilibrium iterations at system level. An additional 'global' iterative loop is not required in this procedure.

When considering thin-walled pipes under a pressure load, such as submerged pipes, the radial stress can be disregarded in relation to the stress in circumferential direction. Additionally, the pressure is considered to be a prescribed load, hence the stress in circumferential direction, or hoop stress, is prescribed and should not vary during the return mapping algorithm. Only an axial stress increment and a shear stress increment vary. A return mapping algorithm for isotropic hardening material that exactly fulfils these constraints is presented in this chapter. New in this algorithm is the assumption that the hoop stress is prescribed and cannot vary.

\subsubsection{Return mapping algorithm}

A pipe can be subject to a torsional moment $T$, bending moments $M_{\mathrm{y}}$ and $M_{\mathrm{z}}$, and an axial force $N$. These loads are indicated in Figure 6.1. Additionally, it can be subject to external or internal pressure $p$. The pipe is considered long and slender, such that the cross-section remains perpendicular to the axial axis. Therefore, shear forces are not considered.

Figure 6.1 also shows the stress state of a material cube. These stresses are determined with the dedicated return mapping algorithm for thin-walled pipes. In this section the assumptions for the return mapping algorithm for thin-walled pipes are introduced. These assumptions do not directly lead to a simplification 

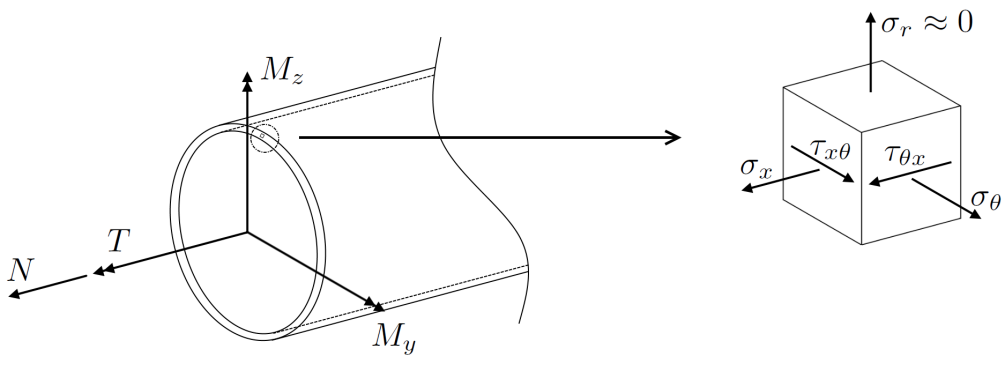

Figure 6.1: Loads on a pipe and resulting stresses.

of the return mapping procedure. This will be shown in the derivation of the return mapping algorithm.

Because the relation between the internal forces and the displacements is nonlinear, an iterative method is required to determine force equilibrium at an increment. When the Newton-Raphson procedure is used as iterative method, a consistently derived stiffness matrix ensures quadratic convergence near equilibrium. This requires an elasto-plastic tangent, which is the derivative of the stress increments to the strain increments. The consistent elasto-plastic tangent is derived at the end of this section.

\section{Assumptions for a thin-walled pipe}

Euler backward stress integration is applied, which means that the stresses are evaluated at the end of an increment. The stress state is known at the start of the increment and unknown at the end of the increment. The prescribed strain increment consists of an axial strain increment $\Delta \varepsilon_{\mathrm{x}}$ and a shear strain increment $\Delta \gamma_{x \theta}$.

$$
\Delta \varepsilon=\left[\begin{array}{ll}
\Delta \varepsilon_{\mathrm{x}} & \Delta \gamma_{x \theta}
\end{array}\right]^{\mathrm{T}}
$$

In the remainder of this chapter the subscript of the shear strain increment is omitted, since the shear strains on other planes are zero. The strain increments in circumferential direction $\Delta \varepsilon_{\theta}$ and radial direction $\Delta \varepsilon_{\mathrm{r}}$ are unknown.

It is assumed that the pipe wall is thin, from which it follows that the radial stress is much smaller than the hoop stress. This leads to a plane stress assumption where the radial stress is zero: $\sigma_{\mathrm{r}}=0$. The hoop stress for a thin-walled pipe is assumed constant over the wall thickness and is equal to:

$$
\sigma_{\theta}=\frac{\left(p^{\mathrm{int}}-p^{\mathrm{ext}}\right) D}{2 t}
$$

Here, $p^{\text {int }}$ and $p^{\text {ext }}$ are the internal and external pressure, $D$ is the pipe diameter and $t$ is the wall thickness. Equation (6.2) shows that the hoop stress at the end of 
the increment is determined by the pressure, which is considered to be a prescribed external load. The hoop stress will therefore not change when plastic deformation occurs, thus the hoop stress increment $\Delta \sigma_{\theta}$ is prescribed. The axial and shear stress increments are unknown:

$$
\Delta \boldsymbol{\sigma}=\left[\begin{array}{ll}
\Delta \sigma_{\mathrm{x}} & \Delta \tau_{x \theta}
\end{array}\right]^{\mathrm{T}}
$$

As for the shear strain, the subscript of the shear stress increment is omitted in the remainder of this chapter.

Isotropic plasticity with nonlinear hardening and plastic incompressibility is assumed, such that the hydrostatic stress increment is determined by the strain increments and bulk modulus $K$ :

$$
\Delta \sigma^{\mathrm{h}}=K\left(\Delta \varepsilon_{\mathrm{x}}+\Delta \varepsilon_{\theta}+\Delta \varepsilon_{\mathrm{r}}\right)
$$

The Von Mises yield criterion is assumed:

$$
\phi=\sigma_{\mathrm{vm}}^{2}-\sigma_{\mathrm{y}}^{2}\left(\epsilon^{\mathrm{P}}\right)=0
$$

Here, $\sigma_{\mathrm{y}}$ is the yield stress, which is a function of the equivalent plastic strain $\epsilon^{\mathrm{p}}$. $\sigma_{\mathrm{vm}}$ is the equivalent Von Mises stress, which is a function of the stress increments in Equations (6.3). These increments are functions of the increment of equivalent plastic strain. For plane stress, $\sigma_{\mathrm{vm}}$ is given by:

$$
\left(\sigma_{\mathrm{vm}}^{n+1}\right)^{2}=\left(\sigma_{\mathrm{x}}^{n}+\Delta \sigma_{\mathrm{x}}\right)^{2}-\left(\sigma_{\mathrm{x}}^{n}+\Delta \sigma_{\mathrm{x}}\right) \sigma_{\theta}^{n+1}+\left(\sigma_{\theta}^{n+1}\right)^{2}+3\left(\tau^{n}+\Delta \tau\right)^{2}
$$

The principle of normality states that the rate of plastic strain must be perpendicular to the yield surface, which leads to the Prandtl-Reuss equation:

$$
\dot{\varepsilon^{\mathrm{p}}}=\dot{\lambda} \frac{\partial \phi}{\partial \boldsymbol{\sigma}} \quad \rightarrow \quad \dot{\varepsilon^{\mathrm{p}}}=3 \dot{\lambda} s
$$

In the incremental algorithm Euler backward stress integration is used, such that the deviatoric stress $s$ is evaluated at the end of the increment. Furthermore, the plastic multiplier is shortened from $\Delta \lambda$ to $\lambda$. This leads to the incremental formulation of the Prandtl-Reuss equation:

$$
\Delta \varepsilon^{\mathrm{p}}=3 \lambda s^{n+1}
$$

Here, $\Delta \varepsilon^{\mathrm{p}}$ is the increment of plastic strain, $\lambda$ is the plastic multiplier and $\boldsymbol{s}^{n+1}$ is the deviatoric stress at the end of the increment.

\section{Derivation of return mapping algorithm}

In the predictor step a deviatoric trial stress $\boldsymbol{s}^{\text {tr }}$ is defined. For 3D plasticity this trial stress is determined from strains using elasticity theory. For plane stress $\boldsymbol{s}^{\operatorname{tr}}$ 
cannot be directly determined from known strain increments. Instead, $\boldsymbol{s}^{\text {tr }}$ depends on $\Delta \varepsilon_{\theta}$ and $\Delta \varepsilon_{\mathrm{r}}$, which are unknown and depend on the plastic strain increment. Therefore, the deviatoric stress at the end of the increment is implicitly defined as the yet unknown deviatoric trial stress scaled with a return mapping factor $\alpha$ :

$$
s^{n+1}=\alpha s^{\text {tr }}
$$

The components of the deviatoric trial stress are defined in an identical way to those in a $3 \mathrm{D}$ radial return mapping algorithm:

$$
\boldsymbol{s}^{\operatorname{tr}}=s^{n}+2 G \mathbf{P} \Delta \bar{\varepsilon} \quad \text { where } \quad \mathbf{P}=\frac{1}{3}\left[\begin{array}{cccc}
2 & -1 & -1 & 0 \\
0 & 0 & 0 & \frac{3}{2}
\end{array}\right]
$$

Here, $\mathbf{P} \Delta \bar{\varepsilon}$ is a deviatoric strain increment. $\Delta \bar{\varepsilon}$ is a strain increment that consists of four components, of which only $\Delta \varepsilon_{\mathrm{x}}$ and $\Delta \gamma$ are known:

$$
\Delta \bar{\varepsilon}=\left[\begin{array}{llll}
\Delta \varepsilon_{\mathrm{x}} & \Delta \varepsilon_{\theta} & \Delta \varepsilon_{\mathrm{r}} & \Delta \gamma
\end{array}\right]^{\mathrm{T}}
$$

In Equation (6.9) both $\alpha$ and $s^{\text {tr }}$ are dependent on the plastic strain increment. The total stress increment can be determined from the change in deviatoric stress and hydrostatic stress increment:

$$
\Delta \boldsymbol{\sigma}=\alpha \boldsymbol{s}^{\mathrm{tr}}-\boldsymbol{s}^{n}+\Delta \boldsymbol{\sigma}^{\mathrm{h}} \quad \text { where } \quad \Delta \boldsymbol{\sigma}^{\mathrm{h}}=\left[\begin{array}{c}
\Delta \sigma^{\mathrm{h}} \\
0
\end{array}\right]
$$

The stress increments in this equation consist of two components: axial stress and shear stress. The shear stress increment is not directly dependent on the strain increments in $\mathrm{x}-, \theta$ - and r-directions. It can be determined by substituting the deviatoric trial shear stress, the second line of Equation (6.10), in Equation (6.12):

$$
\Delta \tau=\alpha\left(\tau^{n}+G \Delta \gamma\right)-\tau^{n}
$$

The deviatoric trial axial stress depends on the unknown strain increments $\Delta \varepsilon_{\theta}$ and $\Delta \varepsilon_{\mathrm{r}}$, as can be seen in the first line of Equation (6.10). These unknowns can be eliminated using the hydrostatic stress increment $\Delta \sigma^{\mathrm{h}}$, which can be calculated in two ways. Firstly, it can be determined using the bulk modulus as shown in Equation (6.4). Secondly, it can be calculated from the stress increments. Since the stress increment in r-direction is zero, $\Delta \sigma^{\mathrm{h}}$ only depends on the stress increments in $\mathrm{x}$ - and $\theta$-direction, of which $\Delta \sigma_{\theta}$ is known:

$$
\Delta \sigma^{\mathrm{h}}=\frac{1}{3}\left(\Delta \sigma_{\mathrm{x}}+\Delta \sigma_{\theta}\right)
$$

With Equations (6.4) and (6.14), $\Delta \varepsilon_{\theta}$ and $\Delta \varepsilon_{\mathrm{r}}$ can be eliminated from the first line of Equation (6.10):

$$
s_{\mathrm{x}}^{\mathrm{tr}}=s_{\mathrm{x}}^{n}+\frac{2}{3} G\left(3 \Delta \varepsilon_{\mathrm{x}}-\frac{\Delta \sigma_{\mathrm{x}}+\Delta \sigma_{\theta}}{3 K}\right)
$$


Substituting $s_{\mathrm{x}}^{\mathrm{tr}}$ in Equation (6.12) and solving for the axial stress increment yields:

$$
\Delta \sigma_{\mathrm{x}}=\frac{\alpha 3 G \Delta \varepsilon_{\mathrm{x}}+\frac{3}{2}(\alpha-1) s_{\mathrm{x}}^{n}}{1+\alpha \frac{G}{3 K}}+\Delta \sigma_{\theta} \frac{\frac{1}{2}-\alpha \frac{G}{3 K}}{1+\alpha \frac{G}{3 K}}
$$

Next the parameter $\alpha$ is eliminated from Equations (6.13) and (6.16) using the increment of equivalent plastic strain $\Delta \epsilon^{\mathrm{p}}$, which is related to the plastic multiplier $\lambda$. This elimination requires the definition of the increment of equivalent plastic strain in terms of the plastic strain increments:

$$
\left(\Delta \epsilon^{\mathrm{p}}\right)^{2}=\frac{2}{3}\left(\left(\Delta \varepsilon_{\mathrm{x}}^{\mathrm{p}}\right)^{2}+\left(\Delta \varepsilon_{\theta}^{\mathrm{p}}\right)^{2}+\left(\Delta \varepsilon_{\mathrm{r}}^{\mathrm{p}}\right)^{2}+2\left(\Delta \gamma^{\mathrm{p}}\right)^{2}\right)
$$

and the definition of the Von Mises yield stress in terms of deviatoric stresses:

$$
\sigma_{\mathrm{vm}}^{2}=\frac{3}{2}\left(s_{\mathrm{x}}^{2}+s_{\theta}^{2}+s_{\mathrm{r}}^{2}+2 \tau^{2}\right)
$$

The relation between $\alpha$ and $\lambda$ can be determined by substituting Equations (6.17) and (6.18) in Equation (6.8):

$$
\Delta \epsilon^{\mathrm{p}}=2 \lambda \sigma_{\mathrm{vm}}^{n+1}
$$

The corrector step can be written as:

$$
\boldsymbol{s}^{n+1}=\boldsymbol{s}^{\mathrm{tr}}-2 G \Delta \varepsilon^{\mathrm{p}}
$$

Substituting Equations (6.8), (6.9) and (6.19) yields:

$$
\alpha=\frac{1}{1+6 \lambda G}
$$

Equation (6.21) can be used to eliminate return mapping factor $\alpha$ from the stress increments. This means that in this return mapping procedure the predictor and corrector step are interwoven. Substituting Equation (6.21) and Young's modulus $E=9 K G /(3 K+G)$ in Equations (6.13) and (6.16) yields:

$$
\begin{aligned}
\Delta \sigma_{\mathrm{x}} & =E \frac{\Delta \varepsilon_{\mathrm{x}}-3 \lambda s_{\mathrm{x}}^{n}}{1+2 \lambda E}+\Delta \sigma_{\theta} \frac{\nu+\lambda E}{1+2 \lambda E} \\
\Delta \tau & =G \frac{\Delta \gamma-6 \lambda \tau^{n}}{1+6 \lambda G}
\end{aligned}
$$

The only unknown in these equations is $\lambda$, which is a function of $\Delta \epsilon^{\mathrm{p}}$. When there is no plasticity, $\lambda=0$ and Equation (6.22) reduces to:

$$
\Delta \boldsymbol{\sigma}=\left[\begin{array}{cc}
E & 0 \\
0 & G
\end{array}\right] \Delta \varepsilon+\left[\begin{array}{c}
\nu \Delta \sigma_{\theta} \\
0
\end{array}\right]
$$


When plastic deformation occurs, the increment of equivalent plastic strain is solved iteratively from Equations (6.5), (6.6), (6.19) and (6.22) with the NewtonRaphson algorithm. This requires the derivative of $\phi$ to $\Delta \epsilon^{\mathrm{p}}$, which is determined as:

$$
\frac{\partial \phi}{\partial \Delta \epsilon^{\mathrm{p}}}=\boldsymbol{q}^{\mathrm{T}} \frac{\partial \Delta \boldsymbol{\sigma}}{\partial \Delta \epsilon^{\mathrm{p}}}-2 \sigma_{\mathrm{y}} \frac{\partial \sigma_{\mathrm{y}}}{\partial \Delta \epsilon^{\mathrm{p}}}
$$

with

$$
\boldsymbol{q}^{\mathrm{T}}=\left[\begin{array}{ll}
2\left(\sigma_{\mathrm{x}}^{n}+\Delta \sigma_{\mathrm{x}}\right)-\sigma_{\theta}^{n+1} & 6\left(\tau^{n}+\Delta \tau\right)
\end{array}\right]
$$

The stress increments, Equation (6.22), are functions of $\lambda$, which is dependent on the equivalent plastic strain increment. The derivatives of the stress increments to $\Delta \epsilon^{\mathrm{P}}$ can be determined as:

$$
\frac{\partial \Delta \boldsymbol{\sigma}}{\partial \Delta \epsilon^{\mathrm{p}}}=\frac{\partial \Delta \boldsymbol{\sigma}}{\partial \lambda} \frac{\partial \lambda}{\partial \Delta \epsilon^{\mathrm{p}}}=\left[\begin{array}{c}
-E \frac{2 E \Delta \varepsilon_{\mathrm{x}}+3 s_{\mathrm{x}}^{n}-\Delta \sigma_{\theta}(1-2 \nu)}{(1+2 E \lambda)^{2}} \\
-G \frac{6 G \Delta \gamma+6 \tau^{n}}{(1+6 G \lambda)^{2}}
\end{array}\right] \frac{\partial \lambda}{\partial \Delta \epsilon^{\mathrm{P}}}
$$

And the derivative of $\lambda$ to the increment of equivalent plastic strain can be determined from Equation (6.19), using $\sigma_{\mathrm{vm}}=\sigma_{\mathrm{y}}\left(\Delta \epsilon^{\mathrm{P}}\right)$ :

$$
\frac{\partial \lambda}{\partial \Delta \epsilon^{\mathrm{p}}}=\frac{1}{2 \sigma_{\mathrm{y}}}-\frac{\lambda}{\sigma_{\mathrm{y}}} \frac{\partial \sigma_{\mathrm{y}}}{\partial \Delta \epsilon^{\mathrm{P}}}
$$

Any isotropic hardening model can be used to determine the stress-strain curve. Hardening with Ludwik's equation will be used in the examples in this chapter:

$$
\sigma_{\mathrm{y}}=\sigma_{\mathrm{y}}^{0}+H\left(\epsilon^{\mathrm{p}}\right)^{k}
$$

Here, $\sigma_{\mathrm{y}}^{0}$ is an initial yield stress; $H$ and $k$ are Ludwik parameters. The derivative of this equation to $\Delta \epsilon^{\mathrm{p}}$ is:

$$
\frac{\partial \sigma_{\mathrm{y}}}{\partial \Delta \epsilon^{\mathrm{p}}}=k H\left(\epsilon^{\mathrm{p}}\right)^{k-1}
$$

The return mapping algorithm for elasto-plastic deformation of thin-walled pipes is summarised in Algorithm 6.1. In this algorithm a check is made of whether or not the elastically determined stress remains within the yield surface by determining the equivalent Von Mises stress from the elastic stress increment. When the Von Mises stress exceeds the yield stress, plastic deformation occurs. 
Otherwise, deformations remain elastic.

Initialize $\lambda=0$

Determine $\Delta \boldsymbol{\sigma}$ with Equation (6.22)

Determine $\phi(\Delta \boldsymbol{\sigma})$ with Equations (6.5) and (6.6)

if $\phi>0$ then

Plastic deformation

Solve $\Delta \epsilon^{\mathrm{P}}$ iteratively from Equations (6.5), (6.6), (6.19) and (6.22)

Update $\lambda=\frac{\Delta \epsilon^{\mathrm{p}}}{2 \sigma_{\mathrm{y}}}$

Determine $\Delta \boldsymbol{\sigma}$ from Equation (6.22)

else

Elastic deformation

$\Delta \epsilon^{\mathrm{P}}=0$

end

Algorithm 6.1: Return mapping algorithm for thin-walled pipes.

\section{Elasto-plastic tangent}

A consistent stiffness matrix is required to get good convergence in finite element simulations. For the return mapping algorithm, the consistent stiffness matrix is called the elasto-plastic tangent and is derived from the derivative of the stress increments, Equation (6.22), to the strain increments in Equation (6.1):

$$
\mathbf{D}=\frac{\partial \Delta \boldsymbol{\sigma}}{\partial \Delta \varepsilon}=\left.\frac{\partial \Delta \boldsymbol{\sigma}}{\partial \Delta \varepsilon}\right|_{\Delta \epsilon^{\mathrm{p}}=\mathrm{const}}+\frac{\partial \Delta \boldsymbol{\sigma}}{\partial \Delta \epsilon^{\mathrm{p}}} \frac{\partial \Delta \epsilon^{\mathrm{p}}}{\partial \Delta \varepsilon}
$$

When the increment of equivalent plastic strain is constant, $\lambda$ is also constant. This results in a diagonal matrix for the first term of Equation (6.30):

$$
\left.\frac{\partial \Delta \boldsymbol{\sigma}}{\partial \Delta \varepsilon}\right|_{\Delta \epsilon^{\mathrm{p}}=\mathrm{const}}=\left[\begin{array}{cc}
\frac{E}{1+2 \lambda E} & 0 \\
0 & \frac{G}{1+6 \lambda G}
\end{array}\right]
$$

The derivative of the stress increments to the equivalent plastic strain increment is given in Equation (6.26). The derivative of the equivalent plastic strain increment to the strain increment is derived using the derivative of Equation (6.5):

$$
\boldsymbol{q}^{\mathrm{T}} \frac{\partial \Delta \boldsymbol{\sigma}}{\partial \Delta \varepsilon}-2 \sigma_{\mathrm{y}} \frac{\partial \sigma_{\mathrm{y}}}{\partial \Delta \epsilon^{\mathrm{P}}} \frac{\partial \Delta \epsilon^{\mathrm{p}}}{\partial \Delta \varepsilon}=0
$$

Substituting Equation (6.30) and solving for $\partial \Delta \epsilon^{\mathrm{p}} / \partial \Delta \varepsilon$ yields:

$$
\frac{\partial \Delta \epsilon^{\mathrm{p}}}{\partial \Delta \boldsymbol{\varepsilon}}=\frac{\left.\boldsymbol{q}^{\mathrm{T}} \frac{\partial \Delta \boldsymbol{\sigma}}{\partial \Delta \boldsymbol{\varepsilon}}\right|_{\Delta \epsilon^{\mathrm{P}}=\mathrm{const}}}{2 \sigma_{\mathrm{y}} \frac{\partial \sigma_{\mathrm{y}}}{\partial \Delta \epsilon^{\mathrm{P}}}-\boldsymbol{q}^{\mathrm{T}} \frac{\partial \Delta \boldsymbol{\sigma}}{\partial \Delta \epsilon^{\mathrm{P}}}}
$$


The entire derivation results in:

$$
\mathbf{D}=\left(\mathbf{I}+\frac{\frac{\partial \Delta \boldsymbol{\sigma}}{\partial \Delta \epsilon^{\mathrm{p}}} \boldsymbol{q}^{\mathrm{T}}}{2 \sigma_{\mathrm{y}} \frac{\partial \sigma_{\mathrm{y}}}{\partial \Delta \epsilon^{\mathrm{p}}}-\boldsymbol{q}^{\mathrm{T}} \frac{\partial \Delta \boldsymbol{\sigma}}{\partial \Delta \epsilon^{\mathrm{p}}}}\right)\left[\begin{array}{cc}
\frac{E}{1+2 \lambda E} & 0 \\
0 & \frac{G}{1+6 \lambda G}
\end{array}\right]
$$

When there is no plastic deformation, the equivalent plastic strain increment is zero, which leads to $\lambda=0$. Because this increment is also constant, the second term of Equation (6.34) is zero. This reduces the elasto-plastic tangent to the elastic matrix:

$$
\mathbf{D}^{\mathrm{el}}=\left[\begin{array}{cc}
E & 0 \\
0 & G
\end{array}\right]
$$

\subsubsection{Validation}

Eight examples are created to demonstrate and validate the dedicated return mapping algorithm for thin-walled pipes. The validation is done by comparing results of the new algorithm with those of a general radial return mapping algorithm [53, 59], in which the zero stress increments in radial and circumferential direction are enforced by an iterative method.

Both algorithms are implemented in Matlab [37]. In these implementations, stress states are evaluated from a strain input at 24 integration points on a single cross-section of a pipe. Moments and axial forces are determined by integrating numerically over these integration points. Curvature, axial force, torsion and external pressure are prescribed in the examples. Because the algorithms require a strain input, an iterative Matlab function is used to determine the corresponding strains.

In all eight examples a pipe is bent to a curvature of $\kappa=0.02$. Properties of the pipe are shown in Table 2.1. Depending on the example, a pressure, axial force and/or torsion are also applied. Table 6.2 shows which of these additional loads are applied in each example.

Table 6.1: Pipe properties.

\begin{tabular}{lll}
\hline Pipe diameter & $D$ & $559 \mathrm{~mm}$ \\
Wall thickness & $t$ & $21 \mathrm{~mm}$ \\
Young's modulus & $E$ & $207 \mathrm{GPa}$ \\
Poisson's ratio & $\nu$ & 0.3 \\
Initial yield stress & $\sigma_{\mathrm{y}}^{0}$ & $410 \mathrm{MPa}$ \\
Ludwik parameter & $H$ & $800 \mathrm{MPa}$ \\
Ludwik parameter & $k$ & 0.5 \\
\hline
\end{tabular}

For both the new and the general algorithm the results of the eight examples can be seen in Figure 6.2. It is clear from this figure that results obtained with 
the new algorithm are identical to those obtained with the general algorithm.

Table 6.2: Loads: each column represents an example; $\mathrm{x}$ indicates which loads are applied in addition to the prescribed curvature.

\begin{tabular}{lllllllllll}
\hline & & & 1 & 2 & 3 & 4 & 5 & 6 & 7 & 8 \\
\hline Pressure & $p$ & $15 \mathrm{MPa}$ & - & $\mathrm{x}$ & - & - & - & $\mathrm{x}$ & $\mathrm{x}$ & $\mathrm{x}$ \\
Axial force & $N$ & $1000 \mathrm{kN}$ & - & - & $\mathrm{x}$ & - & $\mathrm{x}$ & - & $\mathrm{x}$ & $\mathrm{x}$ \\
Torsion & $T$ & $1000 \mathrm{kNm}$ & - & - & - & $\mathrm{x}$ & $\mathrm{x}$ & $\mathrm{x}$ & - & $\mathrm{x}$ \\
\hline
\end{tabular}

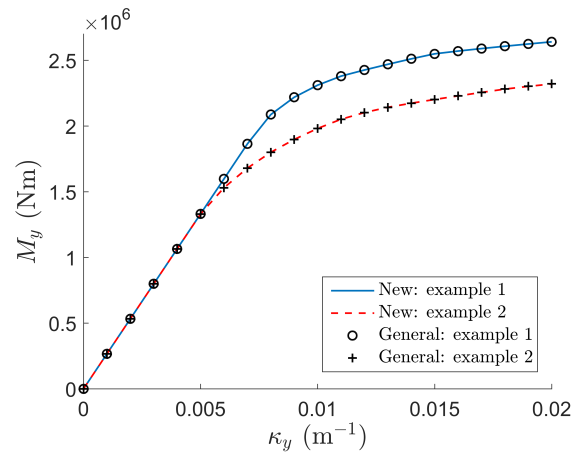

(a) 1: no additional load, 2: pressure.

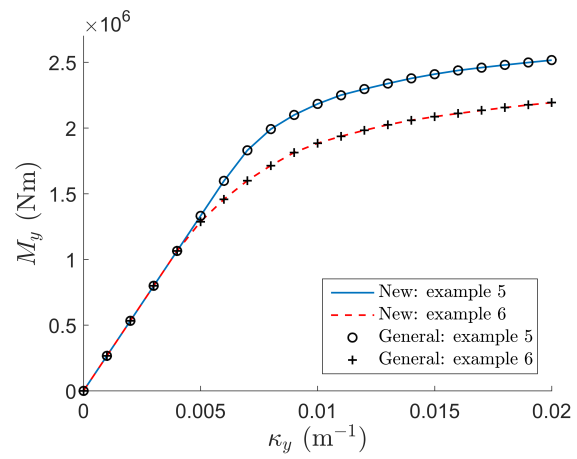

(c) 5: axial force and torsion, 6:pressure and (d) torsion.

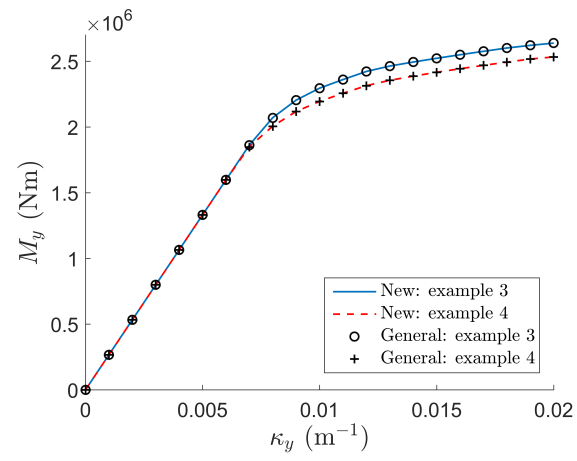

(b) 3: axial force, 4: torsion.

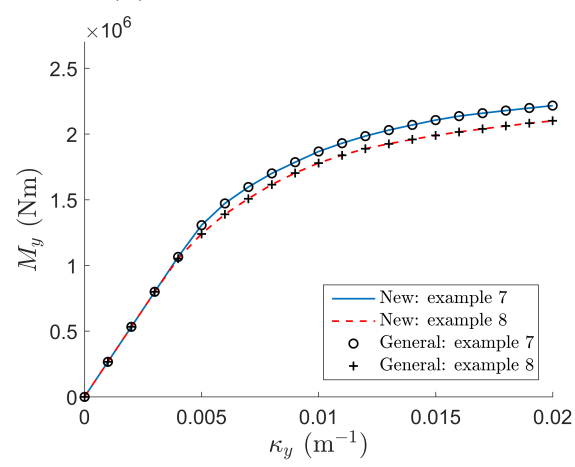

(d) 7: pressure and normal force, 8: pressure, axial force and torsion.

Figure 6.2: Eight examples with varying loads.

Table 6.3 shows the calculation time for both algorithms, subdivided for each example. For Example 1, where no additional loads are applied, the new algorithm requires approximately one third of the computational time of the general procedure. When additional loads are applied, the new algorithm 
requires less than a quarter of the computational time of the general algorithm. Especially when torsion is applied, the number of 'global' iteration loops of the general algorithm increases. It can be concluded that the new algorithm is significantly more efficient than the general algorithm.

Table 6.3: Computational time of the eight examples in seconds.

\begin{tabular}{lllllllll}
\hline & 1 & 2 & 3 & 4 & 5 & 6 & 7 & 8 \\
\hline General (s) & 2.4 & 5.1 & 5.5 & 6.4 & 6.3 & 7.0 & 6.7 & 7.4 \\
New (s) & 0.8 & 1.7 & 1.6 & 2.1 & 1.6 & 1.8 & 1.4 & 1.8 \\
\hline
\end{tabular}

\subsubsection{Discussion}

A return mapping algorithm is radial when the corrector step is in a direction radial to the yield surface. In the new algorithm the trial stress, Equation (6.10), is not directly known because the strain increments $\Delta \varepsilon_{\theta}$ and $\Delta \varepsilon_{\mathrm{r}}$ are unknown. In Equation (6.12) the deviatoric trial stress is scaled with return mapping factor $\alpha$. This defines the corrector step, thus the new return mapping algorithm is radial. This is different from the plane stress return mapping algorithm of Simo and Taylor [55], which is not radial in the plane-stress subspace.

The principle of normality states that the direction of plastic strain must be perpendicular to the yield surface. Equations (6.19) and (6.21) are derived from the principle of normality. This makes sure that the Prandtl-Reuss is enforced and thus that the increment of plastic strain is perpendicular to the yield surface. This algorithm therefore upholds the principle of normality.

Table 6.4: The logarithm of the error norm in a representative increment.

\begin{tabular}{llll}
\hline Iteration number & 1 & 2 & 3 \\
\hline Linear hardening & -1.99 & -5.05 & -11.18 \\
Ludwik hardening & -1.67 & -4.42 & -9.93 \\
\hline
\end{tabular}

In the new algorithm the equivalent plastic strain increment is solved iteratively from the yield criterion, Equation (6.5). In the numerical examples it is observed that when the Newton-Raphson algorithm is used only 1-3 iterations are required. Table 6.4 shows by the logarithm of the error norm in a representative increment that the convergence is quadratic. Because Equation (6.5) is a scalar equation and because the number of iterations is low, the computational time is not significantly influenced by the iterative method. The new algorithm requires significantly less computational time than a general radial return mapping algorithm, in which the zero stress increments in radial and circumferential direction are enforced by a 'global' iterative algorithm. 
In the presented results power hardening with Ludwik's equation was assumed. The new return mapping algorithm can be used for any stress-strain curve, only the derivative of the yield stress to the equivalent plastic strain increment needs to be changed.

\subsection{Implementation in co-rotational elements}

In the previous section stress states were determined at integration points by a return mapping algorithm. In Section 6.2.1, local element forces are determined by integrating stresses resulting from the return mapping algorithm over the volume of a co-rotational pipe element. This integration is split in an integration over the length of the pipe, for which a two point Gauss quadrature rule is used, and an integration over the circumference of the pipe.

Due to the high axial stiffness in comparison wit the bending stiffness, incorrect large stresses can occur during the iterative process. An algorithm that prevents incorrect predictions of plastic deformations is presented in Section 6.2.2.

In Section 2.5 second-order terms resulting from an axial strain component due to the element's curvature were presented for a linear elastic material model. In Section 6.2.3 it is shown that these second-order terms can be adjusted such that they can be used with an elasto-plastic material model.

\subsubsection{Integration over the volume of an element}

The local element forces are determined from stresses $\boldsymbol{\sigma}$ at material points by integrating over the volume of an element:

$$
\boldsymbol{f}_{\mathrm{L}}^{\mathrm{rm}}=\int_{V} \mathbf{Q}^{\mathrm{T}} \mathbf{B}^{\mathrm{T}} \boldsymbol{\sigma} d V
$$

Here, superscript 'rm' indicates that the stresses are determined with the return mapping algorithm. The volume integral can be split into two parts: an integral over the length of the element and an integral over the cross-sectional area. The two-point Gauss quadrature rule [1] is be used to integrate over the length of a pipe element. Matrix $\mathbf{Q}$ relates the local degrees of freedom to the strains at these two Gauss points and matrix $\mathbf{B}$ relates the strains at the Gauss points to strains at integration points on the circumference of the pipe, such that:

$$
\boldsymbol{f}_{\mathrm{L}}^{\mathrm{rm}}=L_{0} \int_{\xi} \mathbf{Q}^{\mathrm{T}} \int_{A} \mathbf{B}^{\mathrm{T}} \boldsymbol{\sigma} d A d \xi
$$

The two-point Gauss quadrature rule involves evaluating the integrand at two gauss points within the integrated domain [1]. The integrated domain is defined by local coordinate $\xi$, which is zero at the first node and one at the second node. 
The positions of the Gauss points $\xi_{1}$ and $\xi_{2}$ are determined by setting the Gauss quadrature equal to an analytical integral of a third order polynomial $p$ :

$$
\int_{0}^{1} p(\xi) d \xi=c_{1} p\left(\xi_{1}\right)+c_{2} p\left(\xi_{2}\right)
$$

Here, $c_{1}$ and $c_{1}$ are constants that are solved from Equation (6.38) together with the Gauss points. This makes the Gauss quadrature approximation of a function $f$ equal to:

$$
\int_{0}^{1} f(\xi) d \xi \approx \frac{1}{2} f\left(\frac{1}{2}-\frac{1}{2 \sqrt{3}}\right)+\frac{1}{2} f\left(\frac{1}{2}+\frac{1}{2 \sqrt{3}}\right)
$$

For each element the local degrees of freedom $\boldsymbol{p}_{\mathrm{L}}$ are known, as shown in Section 2.1.1. The six rotational degrees of freedom are non-commutative, thus it is not possible to determine a rotational-increment. Therefore, increments are determined for the element curvatures, axial strain and the angle of torsional twist per unit length:

$$
\mathcal{E}^{n+1}=\mathbf{Q}_{\mathrm{A}} \boldsymbol{p}_{\mathrm{L}} \rightarrow\left[\begin{array}{c}
\kappa_{\mathrm{y} 1} \\
\kappa_{\mathrm{z} 1} \\
\varepsilon \\
\varphi \\
\kappa_{\mathrm{y} 2} \\
\kappa_{\mathrm{z} 2}
\end{array}\right]^{n+1}=\frac{1}{L_{0}}\left[\begin{array}{ccccccc}
0 & -4 & 0 & 0 & 0 & -2 & 0 \\
0 & 0 & -4 & 0 & 0 & 0 & -2 \\
0 & 0 & 0 & 1 & 0 & 0 & 0 \\
-1 & 0 & 0 & 0 & 1 & 0 & 0 \\
0 & 2 & 0 & 0 & 0 & 4 & 0 \\
0 & 0 & 2 & 0 & 0 & 0 & 4
\end{array}\right]\left[\begin{array}{c}
\psi_{1} \\
\theta_{1} \\
\phi_{1} \\
u_{\mathrm{L}} \\
\psi_{2} \\
\theta_{2} \\
\phi_{2}
\end{array}\right]^{n+1}
$$

Here, $\mathbf{Q}_{\mathrm{A}}$ is determined from the first and second derivatives of the interpolation functions in Equation (2.29). The increment of $\mathcal{E}$ can simply be determined by subtracting the curvatures and strains of the previous increment.

$$
\Delta \mathcal{E}=\mathrm{Q}_{\mathrm{A}} \boldsymbol{p}_{\mathrm{L}}-\mathcal{E}^{n}
$$

The increments at each Gauss point can be calculated by:

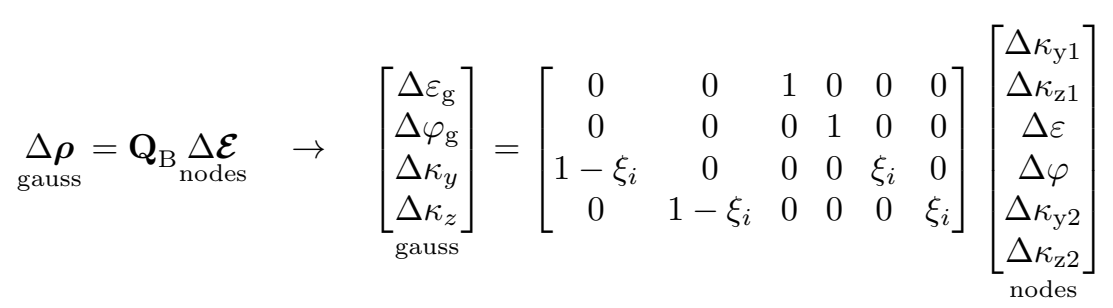

Here, $\xi_{i}$ indicates the position of the first or the second Gauss point. The increments at each Gauss point can be determined directly from the local degrees of freedom:

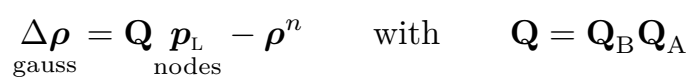


At each Gauss point a number of integration points are defined along the circumference of the pipe, as shown in Figure 6.3. At each integration point stresses are determined with the dedicated return mapping algorithm for thin-walled pipes.

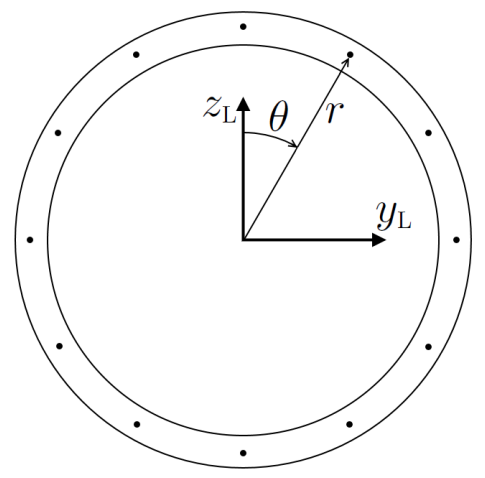

Figure 6.3: Integration points on the cross-section of a pipe.

The local $y_{\mathrm{L}^{-}}$and $z_{\mathrm{L}}$-coordinates of the integration points are defined as:

$$
\begin{aligned}
& y_{j}=r \cos \theta_{j} \\
& z_{j}=r \sin \theta_{j}
\end{aligned}
$$

Here, $j$ indicates the integration point number, $\theta_{j}$ are angles from zero to $2 \pi$ and $r$ is the radius of the circle on which the integration points are positioned. This radius is determined such that the bending moments that result from the numerical integration over the cross-section is identical to the analytically determined bending moments. This can be achieved by determining the moment of inertia by integrating over the cross-section:

$$
I=\sum_{j=1}^{n} z_{j}^{2} \Delta A_{j}=\sum_{j=1}^{n} r^{2} \sin ^{2}\left(\theta_{j}\right) \frac{A}{n}
$$

Here, $n$ is the number of integration points along the circumference of the pipe and $\Delta A_{j}$ is the area per integration point: $A / n$. When $\theta_{j}$ is zero at the top of the pipe and $n \leq 3$, the summation is $\sum_{j=1}^{n} \sin ^{2}\left(\theta_{j}\right) / n$ is equal to $1 / 2$, which leads to:

$$
I=\frac{A}{2} r^{2}
$$

The moment of inertia $I$ and cross-sectional area $A$ are known analytically. Radius $r$ can be solved from Equation (6.46). Substituting the analytical expressions for 
$I$ and $A$ shows that radius $r$ is between inner radius $r_{\text {in }}$ and outer radius $r_{\text {out }}$ :

$$
r=\sqrt{\frac{2 I}{A}}=\sqrt{\frac{1}{2}\left(r_{\mathrm{out}}^{2}+r_{\mathrm{in}}^{2}\right)}
$$

For each integration point the strain increment can be determined:

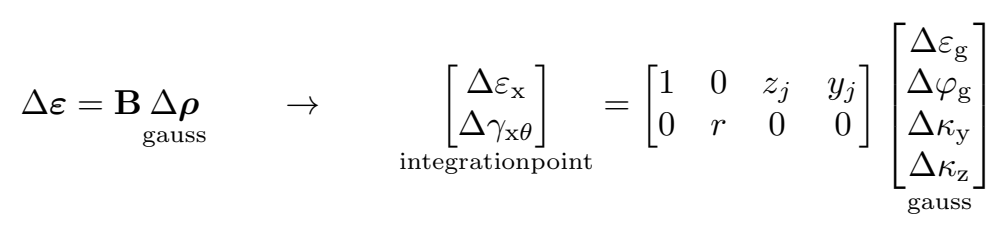

The stresses are determined from this strain increment using the return mapping procedure described in Section 6.1. The element forces are determined by Equation (6.37). In this equation the integral over the area can be calculated numerically as a sum over all integration points on the circumference:

$$
\int_{A} \mathbf{B}^{\mathrm{T}} \boldsymbol{\sigma} d A \approx \sum_{j=1}^{n} \mathbf{B}^{\mathrm{T}} \boldsymbol{\sigma} \Delta A_{j}=\sum_{j=1}^{n}\left[\begin{array}{cc}
1 & 0 \\
0 & r \\
z_{j} & 0 \\
y_{j} & 0
\end{array}\right]\left[\begin{array}{c}
\sigma_{\mathrm{x}} \\
\tau
\end{array}\right] \Delta A_{j}
$$

The integral over the length of the element can be determined using the Gauss quadrature rule in Equation (6.39):

$$
\boldsymbol{f}_{\mathrm{L}}^{\mathrm{rm}}=\frac{L_{0}}{2}\left[\mathbf{Q}^{\mathrm{T}} \sum_{j=1}^{n} \mathbf{B}^{\mathrm{T}} \boldsymbol{\sigma} \Delta A\right]_{\xi_{1}}+\frac{L_{0}}{2}\left[\mathbf{Q}^{\mathrm{T}} \sum_{j=1}^{n} \mathbf{B}^{\mathrm{T}} \boldsymbol{\sigma} \Delta A\right]_{\xi_{2}}
$$

These local forces are a function of the element's degrees of freedom, thus the Newton-Raphson algorithm requires a local stiffness matrix. This stiffness matrix is derived by the variation of $\boldsymbol{f}_{\mathrm{L}}$. In Equation (6.50), only $\boldsymbol{\sigma}$ is dependent on the element's degrees of freedom. All other terms remain constant. The variation $\delta \boldsymbol{\sigma}$ is determined in Section 6.1:

$$
\delta \boldsymbol{\sigma}=\mathbf{D} \delta \varepsilon
$$

The variation $\delta \varepsilon$ can be determined from Equations (6.43) and (6.48):

$$
\delta \varepsilon=\mathbf{B Q} \delta \boldsymbol{p}_{\mathrm{L}}
$$

The local stiffness matrix is determined by substituting Equations (6.51) and (6.52) in the variation $\boldsymbol{f}_{\mathrm{L}}$ :

$$
\mathbf{K}_{\mathrm{L}}^{\mathrm{rm}}=\frac{L_{0}}{2}\left[\mathbf{Q}^{\mathrm{T}} \sum_{j=1}^{n}\left(\mathbf{B}^{\mathrm{T}} \mathbf{D B}\right) \mathbf{Q} \Delta A\right]_{\xi_{1}}+\frac{L_{0}}{2}\left[\mathbf{Q}^{\mathrm{T}} \sum_{j=1}^{n}\left(\mathbf{B}^{\mathrm{T}} \mathbf{D} \mathbf{B}\right) \mathbf{Q} \Delta A\right]_{\xi_{2}}
$$


In Chapter 2 the local force vector had size 12 and the local stiffness matrix had size $12 \times 7$. For readability it is chosen to use local force vectors of size 7 and local stiffness matrices of size $7 \times 7$ in this chapter. These force vectors and stiffness matrices can be changed in size by:

$$
\boldsymbol{f}^{12 \times 1}=\mathbf{S}^{\mathrm{T}} \boldsymbol{f}^{7 \times 1}
$$

where

$$
\mathbf{S}=\left[\begin{array}{cccccccccccc}
0 & 0 & 0 & 1 & 0 & 0 & 0 & 0 & 0 & 0 & 0 & 0 \\
0 & 0 & -\frac{1}{L_{0}} & 0 & 1 & 0 & 0 & 0 & \frac{1}{L_{0}} & 0 & 0 & 0 \\
0 & \frac{1}{L_{0}} & 0 & 0 & 0 & 1 & 0 & -\frac{1}{L_{0}} & 0 & 0 & 0 & 0 \\
-1 & 0 & 0 & 0 & 0 & 0 & 1 & 0 & 0 & 0 & 0 & 0 \\
0 & 0 & 0 & 0 & 0 & 0 & 0 & 0 & 0 & 1 & 0 & 0 \\
0 & 0 & -\frac{1}{L_{0}} & 0 & 0 & 0 & 0 & 0 & \frac{1}{L_{0}} & 0 & 1 & 0 \\
0 & \frac{1}{L_{0}} & 0 & 0 & 0 & 0 & 0 & -\frac{1}{L_{0}} & 0 & 0 & 0 & 1
\end{array}\right]
$$

A stiffness matrix of size $12 \times 7$ can be obtained with:

$$
\mathbf{K}_{\mathrm{L}}^{12 \times 7}=\mathbf{S}^{\mathrm{T}} \mathbf{K}_{\mathrm{L}}^{7 \times 7}
$$

The effect of element rotation on the forces and the stiffness matrix in global coordinates is exactly the same as already derived for the elastic element in Chapter 2 .

\subsubsection{Algorithm for co-rotational elements}

All algorithms for plastic material behaviour are more time-consuming than those for linear elastic material behaviour. In a dynamic pipe-lay simulation plastic material deformation can occur at the start of the simulation or occasionally during the simulation. In most time steps the material behaviour is linear elastic. Therefore, an algorithm is required that uses the return mapping algorithm only when plastic deformation occurs and uses linear elastic relations when elastic deformation occurs.

In a step where material behaves elastically the local element forces can be determined linearly from the elements strains $\mathcal{E}$ in Equation (6.40):

$$
\boldsymbol{f}_{\mathrm{L}}=\mathcal{K}^{\mathrm{e}}\left(\mathcal{E}-\mathcal{E}^{\mathrm{p}}\right) \quad \text { with } \quad \mathcal{K}^{\mathrm{e}}=\left[\begin{array}{cccccc}
0 & 0 & 0 & -G J & 0 & 0 \\
E I & 0 & 0 & 0 & 0 & 0 \\
0 & E I & 0 & 0 & 0 & 0 \\
0 & 0 & E A & 0 & 0 & 0 \\
0 & 0 & 0 & G J & 0 & 0 \\
0 & 0 & 0 & 0 & E I & 0 \\
0 & 0 & 0 & 0 & 0 & E I
\end{array}\right]
$$


Here, $\mathcal{E}^{\mathrm{p}}$ is a vector with plastic element strains, which represent the residual strains and curvatures after removal of forces and moments:

$$
\mathcal{E}^{\mathrm{p}}=\left[\begin{array}{llllll}
\kappa_{\mathrm{y} 1}^{\mathrm{p}} & \kappa_{\mathrm{z} 1}^{\mathrm{p}} & \varepsilon^{\mathrm{p}} & \varphi^{\mathrm{p}} & \kappa_{\mathrm{y} 2}^{\mathrm{p}} & \kappa_{\mathrm{z} 2}^{\mathrm{p}}
\end{array}\right]^{\mathrm{T}}
$$

When elastic deformation occurs, the plastic element strains remain constant. When plastic deformation occurs, the plastic element strains are recalculated from the element forces obtained with Equation (6.50):

$$
\mathcal{E}^{\mathrm{p}}=\mathcal{E}-\left(\mathcal{K}^{\mathrm{e}}\right)^{-1} \boldsymbol{f}_{\mathrm{L}}^{\mathrm{rm}}
$$

In Section 2.3 it was shown that the Newton-Raphson algorithm is used to find force balance. During this 'global' iterative procedure, axial stresses can become very large in the first iterations. This is caused by the large difference in axial and bending stiffness. These large stresses can incorrectly predict plastic deformation.

To prevent these incorrect predictions, an algorithm is proposed that first assumes linear elastic material behaviour, which is shown in Algorithm 6.2. With this assumption, force balance is determined in the 'global' iterative algorithm. When convergence is reached, a check is made to find out whether or not plastic deformation occurs and $\mathcal{E}^{\text {conv }}$ is set to the result of Equation (6.40). When plastic deformation occurs, the 'global' iterative algorithm continuous and the local forces are computed including plasticity:

$$
\boldsymbol{f}_{\mathrm{L}}=\mathcal{K}^{\text {elastic }}\left(\mathcal{E}^{\text {conv }}-\mathcal{E}^{\mathrm{p}}\right)+\mathcal{K}^{\mathrm{rm}} \Delta \mathcal{E}
$$

Here, $\Delta \mathcal{E}$ is the increment with respect to $\mathcal{E}^{\text {conv }}$ and $\mathcal{K}^{\text {rm }}$ is a stiffness matrix determined in a similar way to that in Equation (6.61):

$$
\mathcal{K}^{\mathrm{rm}}=\frac{L_{0}}{2}\left[\mathbf{Q}^{\mathrm{T}} \sum_{j=1}^{n}\left(\mathbf{B}^{\mathrm{T}} \mathbf{D B}\right) \mathbf{Q}_{\mathrm{B}} \Delta A\right]_{\xi_{1}}+\frac{L_{0}}{2}\left[\mathbf{Q}^{\mathrm{T}} \sum_{j=1}^{n}\left(\mathbf{B}^{\mathrm{T}} \mathbf{D} \mathbf{B}\right) \mathbf{Q}_{\mathrm{B}} \Delta A\right]_{\xi_{2}}
$$

\subsubsection{Second-order terms due to bending and plasticity}

In Section 2.5 the additional axial strain due to the curvature of an element was included in the derivation of the local element forces, restricted to linear elastic deformations. In this section, an investigation is made of how these second-order terms can be applied when plastic material deformation occurs.

In the local element forces, lengthening due to curvature result in second-order terms in the axial force, as shown in Equation (2.68). In this equation $\lambda$ is the 


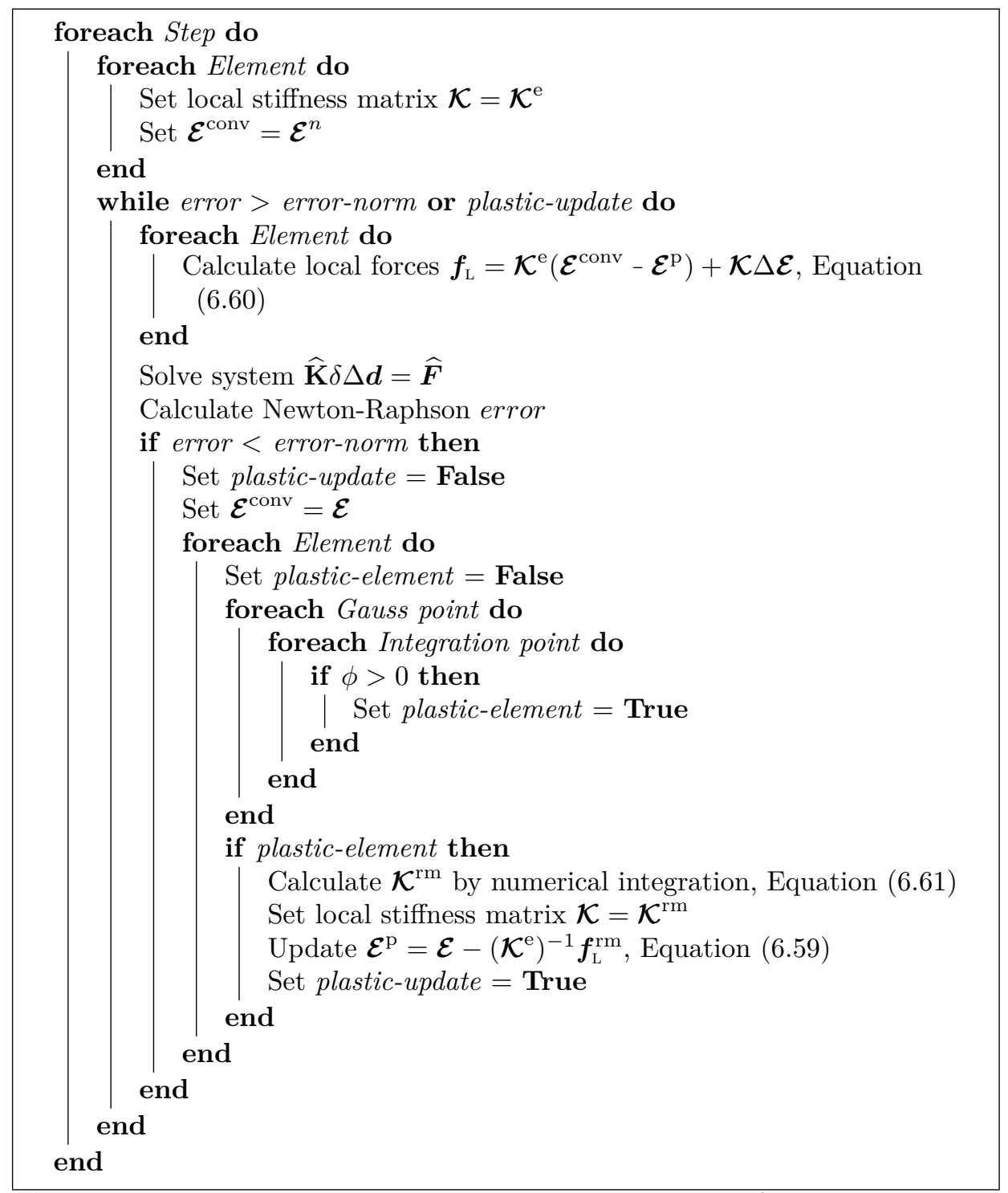

Algorithm 6.2: Plasticity implementation in co-rotational formulation.

additional axial strain, which can be included in $\mathcal{E}$ and in $\boldsymbol{p}_{\mathrm{L}}$ :

$$
\overline{\mathcal{E}}=\left[\begin{array}{c}
\gamma_{1} \\
\kappa_{\mathrm{y} 1} \\
\kappa_{\mathrm{z} 1} \\
\varepsilon+\lambda \\
\gamma_{2} \\
\kappa_{\mathrm{y} 2} \\
\kappa_{\mathrm{z} 2}
\end{array}\right]_{n+1} \quad \overline{\boldsymbol{p}}_{\mathrm{L}}=\left[\begin{array}{c}
\psi_{1} \\
\theta_{1} \\
\phi_{1} \\
u_{\mathrm{L}}+\lambda L_{0} \\
\psi_{2} \\
\theta_{2} \\
\phi_{2}
\end{array}\right]
$$


The local element forces resulting from the return mapping algorithm, $\boldsymbol{f}_{\mathrm{L}}^{\mathrm{rm}}$ from Equation (6.50), can be obtained with $\overline{\boldsymbol{p}}_{\mathrm{L}}$ instead of $\boldsymbol{p}_{\mathrm{L}}$. The plastic element strain can be determined from these element forces:

$$
\overline{\mathcal{E}}^{\mathrm{p}}=\overline{\mathcal{E}}-\mathcal{K}^{-1} \overline{\boldsymbol{f}}_{\mathrm{L}}^{\mathrm{rm}}
$$

With the new plastic strain increment Equation (6.57) can be updated:

$$
\bar{f}_{\mathrm{L}}=\mathcal{K}\left(\overline{\mathcal{E}}-\overline{\mathcal{E}}^{\mathrm{p}}\right)
$$

Lengthening due to curvature also results in second-order terms in the bending moments, as shown in Equation (2.69). In these equations a positive axial force increases the bending stiffness, by terms such as:

$$
N \frac{L_{0}}{30}\left(4 \theta_{1}-\theta_{2}\right)
$$

These terms are geometrical terms, which can be added after the forces are calculated using plasticity:

$$
f_{\mathrm{L}}^{O 2}=\bar{f}_{\mathrm{L}}+N \boldsymbol{b}
$$

where

$$
\boldsymbol{b}=\frac{L_{0}}{30}\left[\begin{array}{lllllll}
0 & 4 \theta_{1}-\theta_{2} & 4 \phi_{1}-\phi_{2} & 0 & 0 & 4 \theta_{2}-\theta_{1} & 4 \phi_{2}-\phi_{1}
\end{array}\right]^{\mathrm{T}}
$$

The stiffness matrix contribution associated with Equation (6.66) is derived by the variation $\delta f_{\mathrm{L}}^{O 2}$ :

$$
\delta \boldsymbol{f}_{\mathrm{L}}^{O 2}=\delta \overline{\boldsymbol{f}}_{\mathrm{L}}+\delta N \boldsymbol{b}+N \delta \boldsymbol{b}
$$

The first term of Equation (6.68) contains the local stiffness matrix. When plastic deformation occurs, this is the local stiffness matrix resulting from the return mapping procedure, as shown in Equation (6.53).

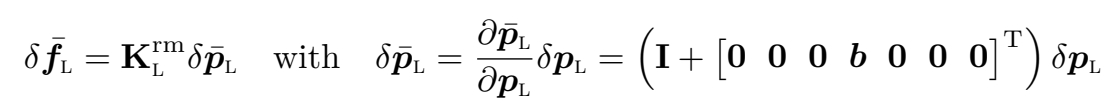

The derivations of the second and third term of Equation (6.68) is straightforward; the resulting stiffness matrix is:

$$
\mathbf{K}_{\mathrm{L}}^{O 2}=\mathbf{K}_{\mathrm{L}}^{\mathrm{rm}}+\mathbf{K}_{\mathrm{L}}^{\mathrm{rm}}\left[\begin{array}{c}
\mathbf{0} \\
\mathbf{0} \\
\mathbf{0} \\
\boldsymbol{b}^{\mathrm{T}} \\
\mathbf{0} \\
\mathbf{0} \\
\mathbf{0}
\end{array}\right]+\boldsymbol{b} \frac{\partial N}{\partial \boldsymbol{p}_{\mathrm{L}}}+N \frac{L_{0}}{30}\left[\begin{array}{ccccccc}
0 & 0 & 0 & 0 & 0 & 0 & 0 \\
0 & 4 & 0 & 0 & 0 & -1 & 0 \\
0 & 0 & 4 & 0 & 0 & 0 & -1 \\
0 & 0 & 0 & 0 & 0 & 0 & 0 \\
0 & 0 & 0 & 0 & 0 & 0 & 0 \\
0 & -1 & 0 & 0 & 0 & 4 & 0 \\
0 & 0 & -1 & 0 & 0 & 0 & 4
\end{array}\right]
$$

Here, $\frac{\partial N}{\partial \boldsymbol{p}_{\mathrm{L}}}$ is the fourth row of $\mathbf{K}_{\mathrm{L}}^{\mathrm{rm}} \frac{\partial \overline{\boldsymbol{p}}_{\mathrm{L}}}{\partial \boldsymbol{p}_{\mathrm{L}}}$. 


\subsection{Closure}

During pipe-laying plastic deformations occur mostly in the pipe sections on the stinger, where the bending radius is the smallest. In this chapter a numerical approach to modelling plastic deformations that can be used with co-rotational pipe elements was presented.

An efficient return mapping procedure for elasto-plastic material behaviour with isotropic hardening in thin-walled pipes was presented. In a thin-walled pipe plane stress can be assumed, with zero stress in radial direction. The hoop stress is determined by the pressure load, and does not change due to plastic deformation. Thus the stress increments in circumferential direction are prescribed and those in radial direction are zero; only the axial and shear stress increments are not zero. A dedicated return mapping procedure with a prescribed hoop stress increment and a zero radial stress increment was derived. In the algorithm, a nonlinear scalar equation is solved iteratively. The new procedure can be used with arbitrary isotropic hardening.

The corrector step of the return mapping procedure is interwoven with the predictor step and the corrector step is radial. At the end of an increment the direction of plastic strain is perpendicular to the yield surface, upholding the principle of normality.

The results of the new procedure are identical to those of a general return mapping algorithm and the computational time is significantly less. The elasto-plastic tangent was presented, such that the procedure can be applied in a finite beam element.

The return mapping approach is implemented in the pipe-lay model with co-rotational elements. When plastic material behaviour occurs, the local element forces depend nonlinearly on the displacements and are determined by numerical integration over the volume of the co-rotational element. At each integration point the return mapping algorithm is applied.

At system level, force balance is determined with the Newton-Raphson iterative algorithm. During this iterative process large axial stresses can occur due to the combination of a high axial stiffness and a low bending stiffness. These large stresses can result in incorrect predictions of plastic deformation. An algorithm that prevents these incorrect predictions was presented.

An element is not straight between its nodes. In Chapter 2 it was shown that taking the curved length into account results in second-order terms in the local element forces. It was shown how these second-order terms can be accounted for when plastic deformation occurs. 



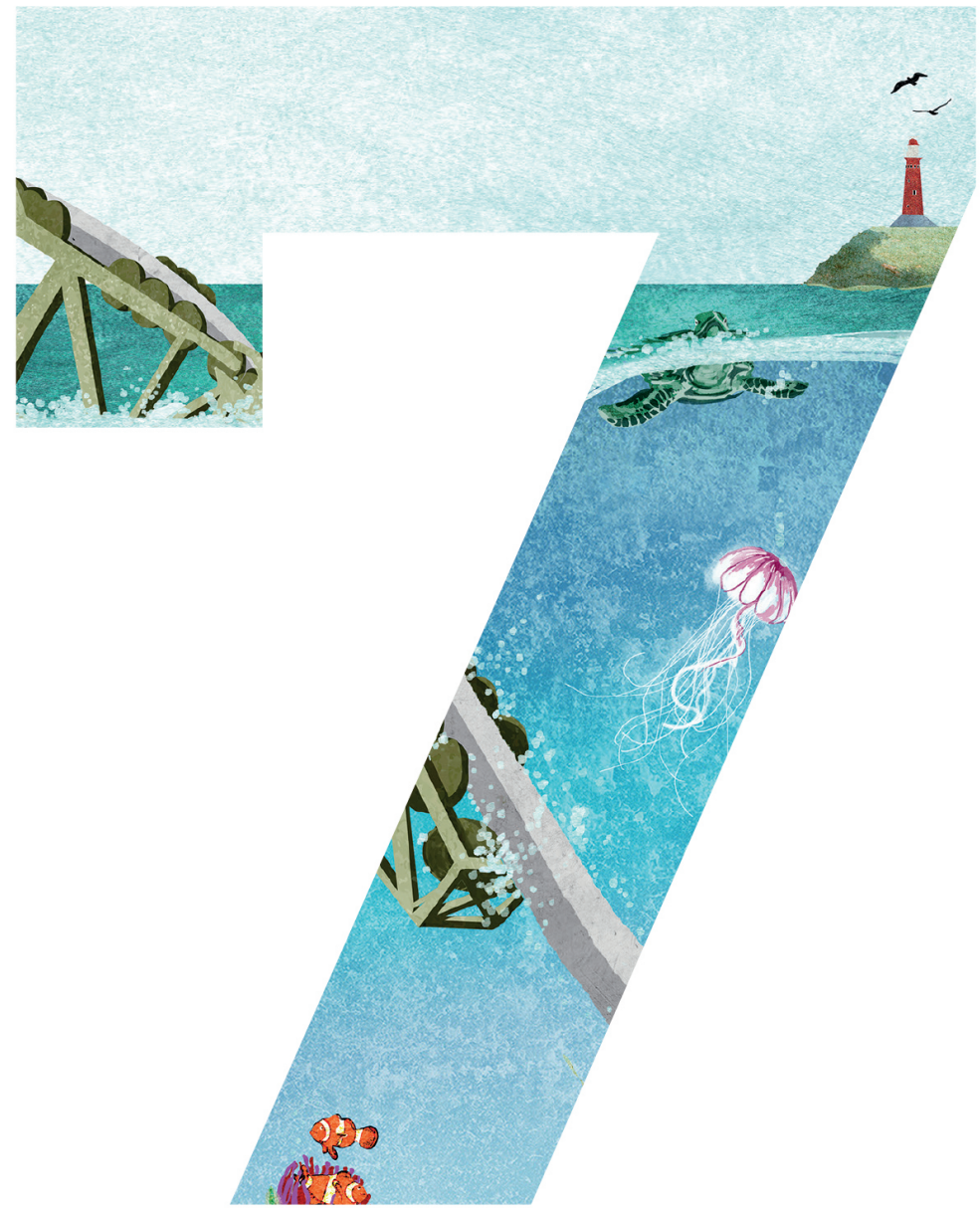




\section{Pipe-lay examples}

In the previous chapters a complete model for numerical analyses of pipe-laying was presented. This pipe-lay model can be used to simulate J-lay and S-lay installation procedures. In this chapter results obtained with the new pipe-lay model are compared with those obtained with Offpipe [36].

Offpipe is widely used standard software, the results of which are considered reliable in industry. The comparison in this chapter gives an indication of the validity of the results obtained with the new pipe-lay model. Because of limitations in the simulations with Offpipe, difference in results does not necessarily mean that results obtained with the new pipe-lay model are incorrect.

In Offpipe buoyancy is modelled with Archimedes' law, resulting in the submerged weight model. This approach disregards the influence of the pipe's curvature and the pressure acting on the pipe's submerged end. The axial forces and strains are computed incorrectly and corrected only in a post-processing step. This approach leads to incorrect results when material behaviour is elasto-plastic or when a free pipe end is involved.

Another limitation of Offpipe is its model for nonlinear material behaviour. In this model a single moment-curvature relation is defined for the entire pipe. This incorrectly assumes a constant axial tension and disregards the effects of torsion and pressure. A residual curvature when unloading is disregarded, resulting in nonlinear elastic behaviour.

In Offpipe the maximum time step size is limited by convergence of the Newton-Raphson algorithm. When the time step is increased, the displacement increment also increases. In nonlinear aspects of the model, such as contact and plastic material behaviour, a large displacement increment can cause problems with convergence.

In the new pipe-lay model a detailed buoyancy model describes the hydrostatic loads on the pipe. This approach includes the influence of the pipe's curvature 
and the pressure acting on the pipe's submerged end, such that axial forces and strains are computed correctly. When possible, the submerged weight model can also be applied.

The dedicated plasticity model of the new pipe-lay model includes a varying axial tension and the effects of torsion and pressure. Additionally, an equivalent plastic strain at material point level ensures a residual curvature when loads are removed. Due to the implementation with residual curvatures, the material behaviour is nonlinear only when plastic deformation occurs. Because the material behaviour is linear in most time steps, the additional computational costs due to the plasticity model are low.

In this work the maximum time step size is not restricted by convergence of the Newton-Raphson algorithm. The contact-state procedure ensures that large time steps do not result in alternating contact states, which would hinder convergence. Incorrect predictions of plastic deformation due to the high axial stiffness are prevented by the implementation of plasticity. The new pipe-lay model is therefore efficient and robust.

With the limitations of Offpipe described above, the comparison with the new pipe-lay model is expected to show similar results for simple examples, such as a J-lay installation with linear elastic material behaviour. The new pipe-lay model overcomes the described limitations. Therefore, differences in results are expected for S-lay simulations with elasto-plastic material behaviour.

In Section 7.1 simulations of pipe-laying with the J-lay method are presented. In these simulations the pipe is in contact with the seabed and is connected to the vessel with a hinge at the end of the pipe. In these simulations the tensioner is not included and the material behaviour is linear elastic.

In Section 7.2 simulations of pipe-laying with the S-lay method are presented. In these simulations the pipe is in contact with the seabed and with roller-boxes on the stinger. The tensioner is included and material behaviour is elasto-plastic.

In Section 7.3 a simulation of the start-up of the execution of a pipe-laying operation with a free hanging pipe end is presented. This simulation is not possible with Offpipe.

\subsection{J-lay procedure}

In this section two linear elastic J-lay examples-J1 and J2-are presented. In the new pipe-lay model buoyancy is applied as described in Section 5.1.2. In Offpipe submerged weight is applied as described in Section 5.1.1. Because there is no plastic material behaviour and no free pipe end, results are expected to be comparable.

The properties of the pipe that is considered in both examples are shown in Table 7.1. There is no fluid inside the pipe, the inner pressure is disregarded. The 
examples differ in water depth, pipe length and bottom tension, as can be seen in Table 7.2. The bottom tension is an external force acting in horizontal direction on the submerged end of the pipe. The magnitude of the bottom tension is equal to that of the thrust of the vessel. The reaction force in the pipe's axial direction at the J-lay hinge is referred to as the top tension.

In these J-lay simulations there is contact between the pipe and the seabed, which is modelled with the Penalty method described in Chapter 4 . The contact-state procedure is not required here, because there are no roller-boxes involved.

Table 7.1: Properties of the pipe used in the J-lay examples.

\begin{tabular}{lll}
\hline Diameter & $D$ & $356 \mathrm{~mm}(14$ inch $)$ \\
Wall thickness & $t$ & $29.3 \mathrm{~mm}$ \\
Young's modulus & $E$ & $207 \mathrm{GPa}$ \\
Poisson's ratio & $\nu$ & 0.3 \\
Density & $\rho$ & $7700 \mathrm{~kg} / \mathrm{m}^{3}$ \\
\hline
\end{tabular}

Table 7.2: Overview of J-lay examples.

\begin{tabular}{lll}
\hline & Example J1 & Example J2 \\
\hline Water depth & $1000 \mathrm{~m}$ & $2000 \mathrm{~m}$ \\
Length & $2000 \mathrm{~m}$ & $3000 \mathrm{~m}$ \\
Bottom tension & $500 \mathrm{kN}$ & $400 \mathrm{kN}$ \\
Number of elements & 100 & 100 \\
\hline
\end{tabular}

\subsubsection{Static simulation}

At the start of a static J-lay simulation the pipe is straight and horizontal. The deformed shape of the pipe is obtained by applying two load steps. In the first load step the bottom tension is applied to the end of the pipe that will be on the seabed. In the second load step gravity and buoyancy loads are applied in 20 substeps. These steps are required for convergence of the Newton-Raphson algorithm. They do not represent physical load steps but can be applied since the material behaviour is linear elastic.

In Figures 7.1a and 7.2a the deformed shape of the pipe resulting from the new pipe-lay model is compared with that resulting from Offpipe for both examples. In both models an equal number of elements is used. Results of the new pipelay model are depicted by a continuous line, results of Offpipe are depicted by a discrete point for every five elements. It can be seen that results of the new pipe-lay model and Offpipe are in excellent agreement. More detailed results can be seen in Table 7.3. The difference between the top tension, departure angle and pipe strains are negligible. 
Figures $7.1 \mathrm{~b}$ and $7.2 \mathrm{~b}$ show strains in a material point of the pipe versus an scoordinate, which is the arc length of the undeformed pipe. In the figures a distinction is made between an axial and a bending strain. The axial strain is determined from the elongation of an element, including second-order terms due to its curvature, as shown in Equation (2.55). The bending strain is determined by multiplying the curvature with the pipe's radius:

$$
\varepsilon_{\mathrm{b}}=\kappa \frac{D}{2}
$$

The resulting axial strains from Offpipe are determined with the post-processing step that is required when using the submerged weight model, as described in Section 5.1.1.

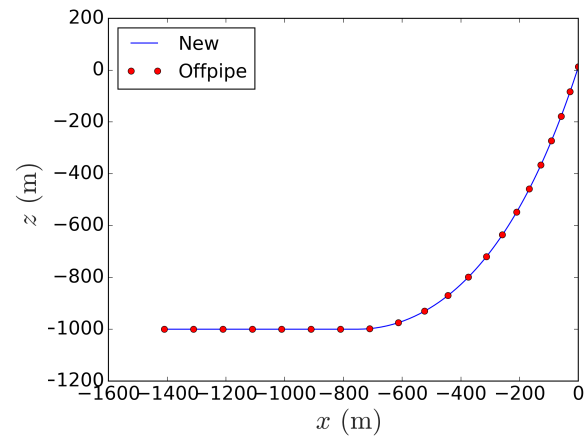

(a) Deformed shape of the pipe.

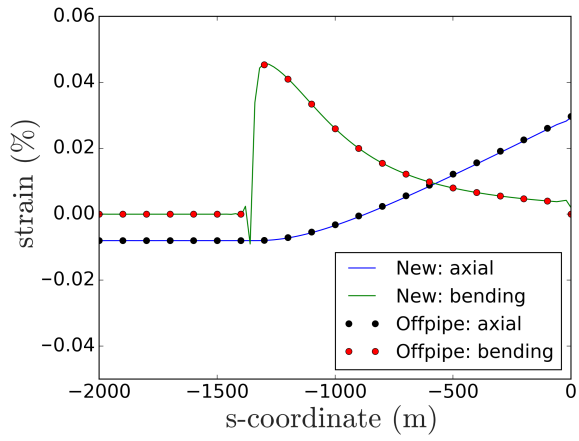

(b) Strains in the pipe.

Figure 7.1: Example J1.

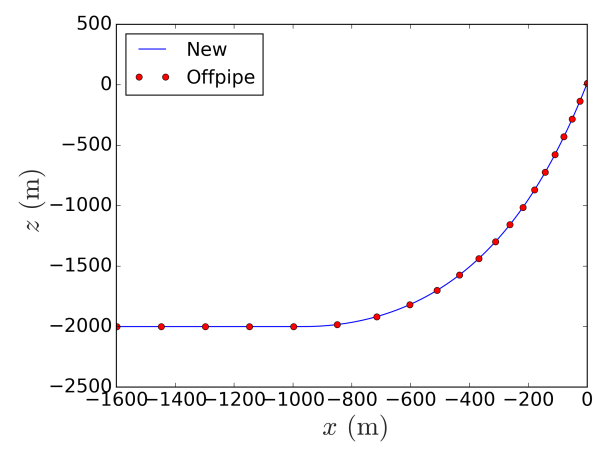

(a) Deformed shape of the pipe.

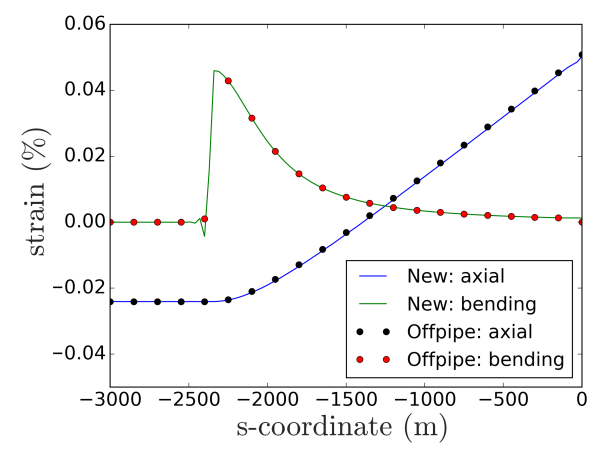

(b) Strains in the pipe.

Figure 7.2: Example J2.

In the results of both models the maximum bending strain is near the seabed. Near 
Table 7.3: Results of static J-lay simulations compared with those of Offpipe.

\begin{tabular}{llllll}
\hline & \multicolumn{2}{c}{ Example J1 } & \multicolumn{2}{c}{ Example J2 } \\
& New & Offpipe & & New & Offpipe \\
\cline { 2 - 3 } \cline { 5 - 6 } Top tension & $1844 \mathrm{kN}$ & $1845 \mathrm{kN}$ & & $3158 \mathrm{kN}$ & $3158 \mathrm{kN}$ \\
Departure angle & $74.1 \mathrm{deg}$ & $74.1 \mathrm{deg}$ & & $80.9 \mathrm{deg}$ & $80.9 \mathrm{deg}$ \\
Maximum bending strain & $0.046 \%$ & $0.045 \%$ & & $0.046 \%$ & $0.045 \%$ \\
Maximum axial strain & $0.029 \%$ & $0.030 \%$ & & $0.050 \%$ & $0.051 \%$ \\
\hline
\end{tabular}

the J-lay hinge the bending strain decreases. A difference in results is noticeable at the touch down point. The new pipe-lay model shows a small peak in bending strain, which is not noticeable in the results from Offpipe. This small peak is caused by the discretisation of the seabed and completely disappears when the stiffness of the seabed decreases.

\subsubsection{Dynamic simulation}

In this section results from dynamic simulations of the two J-lay examples are presented. These are the same examples as for the static simulation.

In the new pipe-lay model results of dynamic simulations are obtained with numerical time integration by the HHT- $\alpha$ method, as described in Chapter 3 . The initialisation procedure described in Section 3.3 is used to damp high-frequency behaviour at the start of the simulation. Properties of the numerical time integration are shown in Table 7.4.

In the dynamic J-lay simulations displacements of the J-lay hinge are prescribed by the response of the pipe-lay vessel to a regular wave, as described in Section 5.3.1. For both examples properties of this wave are shown in Table 7.4. The resulting displacements of the J-lay hinge are shown in Figures 7.3a and 7.4a for respectively examples $\mathrm{J} 1$ and $\mathrm{J} 2$.

Table 7.4: Simulation and environmental properties of the dynamic J-lay examples.

\begin{tabular}{lll}
\hline Time step & $\Delta t$ & $1 \mathrm{~s}$ \\
Simulation time & $T_{\text {total }}$ & $100 \mathrm{~s}$ \\
Initial period & $T_{\mathrm{ini}}$ & $20 \mathrm{~s}$ \\
Numerical damping & $\alpha$ & 0.03 \\
Water density & $\rho_{\mathrm{w}}$ & $1025 \mathrm{~kg} / \mathrm{m}^{3}$ \\
Wave height & $h$ & $1 \mathrm{~m}$ \\
Wave period & $T$ & $7 \mathrm{~s}$ \\
\hline
\end{tabular}

The deformed shape of the pipe at time zero is determined from the result of the 
corresponding static simulations of Section 7.1.1. In the result of the shape of the pipe, the node of the pipe's end that is in contact with the seabed is fixed in its place. At time zero the position of the J-lay hinge is not the same as its position in the static simulation. The position of the J-lay hinge is determined by the displacements and rotations of the vessel, which are determined by the encountering wave and the RAO's. The J-lay hinge is moved to the correct position in an additional static load step.

An overview of the results from the simulations of both examples with the new pipe-lay model and Offpipe is presented in Table 7.5. This overview includes the minimum and maximum top tension and maximum strains in the pipe. The differences between these results are negligible small.

Table 7.5: Results of dynamic J-lay simulations compared with those of Offpipe.

\begin{tabular}{llllll}
\hline & \multicolumn{2}{c}{ Example J1 } & & \multicolumn{2}{c}{ Example J2 } \\
& New & Offpipe & & New & Offpipe \\
\cline { 2 - 3 } \cline { 5 - 6 } Max top tension & $1893 \mathrm{kN}$ & $1900 \mathrm{kN}$ & & $3162 \mathrm{kN}$ & $3164 \mathrm{kN}$ \\
Min top tension & $1793 \mathrm{kN}$ & $1785 \mathrm{kN}$ & & $2962 \mathrm{kN}$ & $2954 \mathrm{kN}$ \\
Max bending strain & $0.049 \%$ & $0.048 \%$ & & $0.059 \%$ & $0.058 \%$ \\
Max axial strain & $0.030 \%$ & $0.031 \%$ & & $0.050 \%$ & $0.051 \%$ \\
\hline
\end{tabular}

Figures 7.3 and 7.4 show more detailed results of examples J1 and J2. In Figures $7.3 \mathrm{~b}$ and $7.4 \mathrm{~b}$, the minimum and maximum axial and bending strain resulting from the new pipe-lay model are compared with those resulting from Offpipe. It can be seen that the results are in excellent agreement.

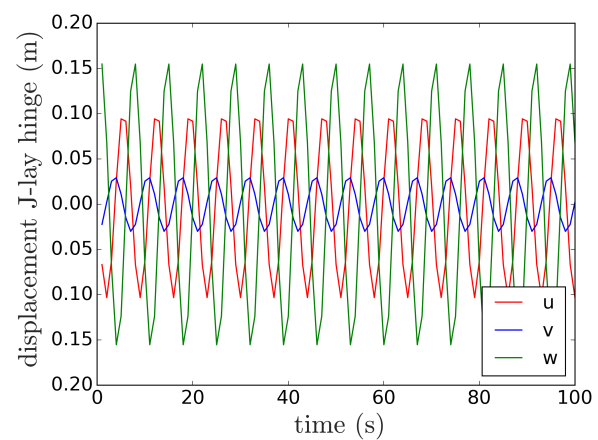

(a) Prescribed displacements of the J-lay (b) Maximum bending and axial strains in hinge.

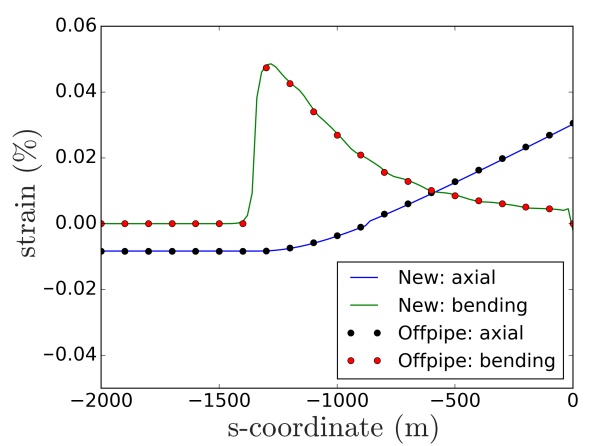

the pipe.

Figure 7.3: Example J1.

In the new pipe-lay model buoyancy is applied as described in Section 5.1.2. In Offpipe submerged weight is applied as described in Section 5.1.1. Since the results 


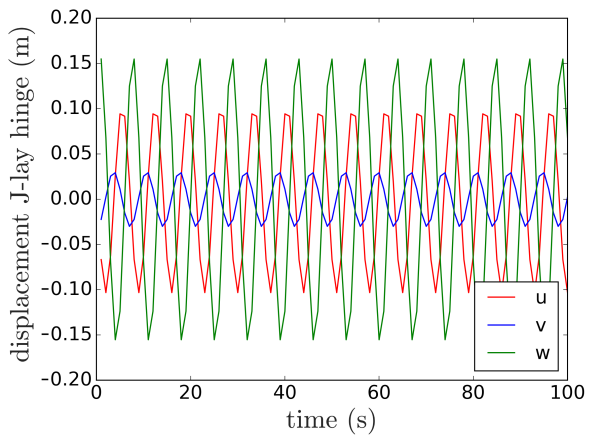

(a) Prescribed displacements of the J-lay hinge.

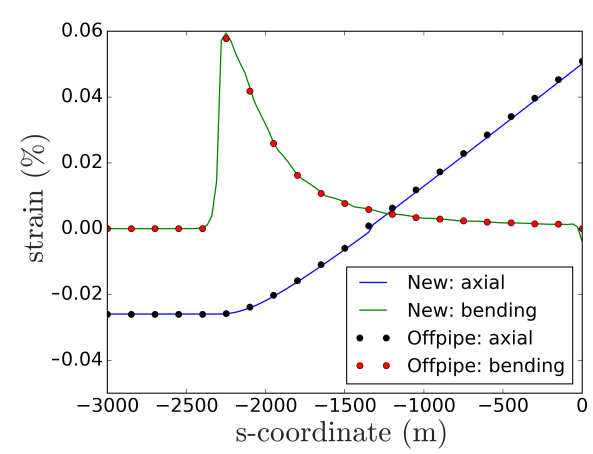

(b) Maximum bending and axial strains in the pipe.

Figure 7.4: Example J2.

of both models are in agreement, it can be concluded that the computationally less expensive submerged weight model can be applied for J-lay simulations with linear elastic material behaviour. Applying submerged weight in the new pipe-lay model reduces computational costs by approximately $15 \%$.

In the new pipe-lay model and in Offpipe the time step is set to $\Delta t=1 \mathrm{~s}$, which is the maximum time step size determined in Section 5.4.1. When an equal time step is used, computational costs of both model are approximately equal. It can be concluded that Offpipe and the new pipe-lay model are equally accurate and efficient for J-lay simulations with linear elastic material behaviour.

\subsection{S-lay procedure}

In this section two S-lay examples named S1 and S2 are presented. In example $\mathrm{S} 1$ material behaviour is linear elastic and in example S2 material behaviour is elasto-plastic. Both examples are based on actual pipe-lay projects of Allseas [2].

Properties of the pipes that are considered in the examples are shown in Table 7.6. The examples also differ in water depth, pipe length, bottom tension and number of elements, as can be seen in Table 7.7.

In these S-lay simulations there is contact between the pipe and the seabed and between the pipe and the roller-boxes. The contact-state procedure described in Section 4.4 is applied. The pipe is connected to the vessel at the tensioner, as described in Section 4.5. The reaction force in axial direction at the tensioner is referred to as the top tension.

In example $\mathrm{S} 2$ material behaviour is elasto-plastic. The material of the pipe is API-5L-X65, where 'API-5L' refers to a material specification for pipes that are 
Table 7.6: Properties of the pipes used in the S-lay examples.

\begin{tabular}{llll}
\hline & & Example S1 & Example S2 \\
\hline Diameter & $D$ & $273 \mathrm{~mm}(10 \mathrm{inch})$ & $813 \mathrm{~mm}(32$ inch $)$ \\
Wall thickness & $t$ & $20.6 \mathrm{~mm}$ & $39 \mathrm{~mm}$ \\
Young's modulus & $E$ & $207 \mathrm{GPa}$ & $207 \mathrm{GPa}$ \\
Poisson's ratio & $\nu$ & 0.3 & 0.3 \\
Density & $\rho$ & $7850 \mathrm{~kg} / \mathrm{m}^{3}$ & $7850 \mathrm{~kg} / \mathrm{m}^{3}$ \\
Yield stress & $\sigma_{\mathrm{y}}^{0}$ & & $415 \mathrm{MPa}$ \\
Hardening modulus & $H$ & & $12 \mathrm{GPa}$ \\
\hline
\end{tabular}

Table 7.7: Overview of S-lay examples.

\begin{tabular}{lll}
\hline & Example S1 & Example S2 \\
\hline Water depth & $1710 \mathrm{~m}$ & $2200 \mathrm{~m}$ \\
Length & $2500 \mathrm{~m}$ & $3800 \mathrm{~m}$ \\
Stinger radius & $80 \mathrm{~m}$ & $140 \mathrm{~m}$ \\
Bottom tension & $115 \mathrm{kN}$ & $2470 \mathrm{kN}$ \\
Number of elements & 250 & 215 \\
\hline
\end{tabular}

suitable for conveying gas, water and oil. 'X65' refers to a material with a yield strength of $450 \mathrm{MPa}$.

In Offpipe material behaviour is modelled by a nonlinear relation between bending moment and curvature, known as the Ramberg-Osgood equation. In Offpipe [36] the corresponding Ramberg-Osgood equation is defined by:

$$
\frac{\kappa}{\bar{\kappa}}=\frac{M}{\bar{M}}+A\left(\frac{M}{\bar{M}}\right)^{B} \quad \text { with } \quad \bar{\kappa}=\frac{2 \sigma_{\mathrm{y}}}{E D}, \quad \bar{M}=\frac{2 \sigma_{\mathrm{y}} I}{D}
$$

The input of Offpipe are the parameters of the Ramberg-Osgood equation. These can be obtained by a curve fit of a numerically determined moment-curvature relation in a pre-processing step, using the specified material and an axial force of $7230 \mathrm{kN}$. This axial force is equal to the maximum axial force in the static simulation.

For the pipe in example S2 the resulting Ramberg-Osgood parameters are $A=$ 0.2107 and $B=9.4414$. The corresponding curve is shown in Figure $7.5 \mathrm{a}$. In Offpipe this curve does not change when the load is removed.

In the new pipe-lay model plastic material behaviour is modelled by the dedicated plasticity model with linear hardening, as described in Chapter 6. To obtain the best comparable results, the hardening parameters $\sigma_{\mathrm{y}}^{0}$ and $H$ chosen are such that the moment-curvature graph obtained in the pre-processing step is approximately equal to that obtained in the dedicated plasticity model. 


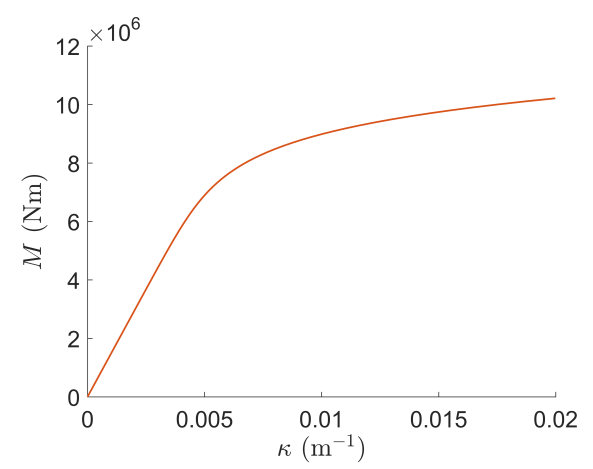

(a) Ramberg-Osgood.

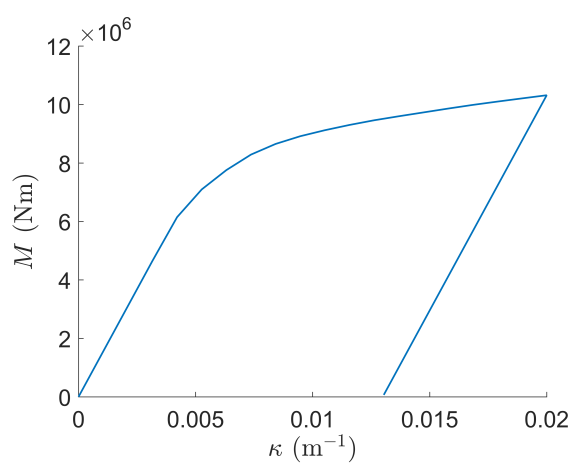

(b) Numerical integration.

Figure 7.5: Moment curvature graph.

In Figure 7.5b a graph obtained with hardening parameters from Table 7.6 and an axial force of $7230 \mathrm{kN}$ is shown. When the load is removed, the material behaves elastically.

In Offpipe the moment-curvature relation remains constant in the entire simulation. In reality and in the new pipe-lay model the moment-curvature relation changes when, for example, the axial force increases. In Figure 7.5 it can also be seen that the new pipe-lay model includes a residual curvature when unloading, which is not included in Offpipe.

\subsubsection{Static simulation}

The deformed shape of the pipe is obtained by the same load steps as for the static J-lay simulations. A relevant difference is that plastic deformations can occur in these S-lay simulations. In reality, the pipe is bent over the stinger while subject to an axial tension. The pipe sections that have passed the stinger are bent back and can have a residual curvature. In the simulation the pipe is bent over the stinger after it is loaded with an axial force. The residual curvature in the pipe can be taken into account, as will be shown in Section 7.3. It is not considered in examples S1 and S2.

Results of example S1 are compared in Table 7.8 and in Figure 7.6. The values compared in the table include departure angle and tip separation. The departure angle is the angle between the horizon and the local $x_{\mathrm{L}}$-axis of the pipe section at the stinger tip. Tip separation is the distance between this pipe section and the stinger tip.

In Figure 7.6a it can be seen that the deformed shape of the pipe resulting from the new pipe-lay model is approximately equal to that from Offpipe. In Figure 7.6b it can be seen that the bending strains at the stinger predicted by Offpipe are higher 


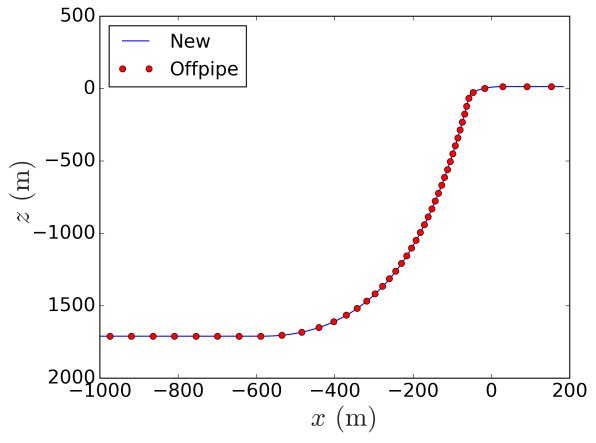

(a) Deformed shape of the pipe.

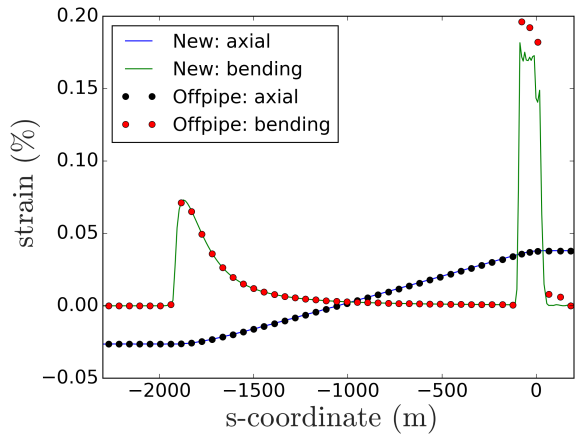

(b) Strains in the pipe.

Figure 7.6: Example S1.

than those predicted by the new pipe-lay model. This is unexpected, because these bending strains are defined by the geometry of the pipe at the stinger, which is identical in both cases.

The differences in bending strains at the stinger can be explained. In the new pipelay model the bending strains are computed directly from the pipe's curvature, as shown in Equation (7.1). In Offpipe the bending strain is computed from the bending moment [36]:

$$
\varepsilon_{\mathrm{b}}=\frac{M D}{2 E I}
$$

Equation (7.3) can be derived from the classic formula for determining the maximum stress in a beam under simple bending. In Offpipe the bending moments are computed including second-order terms, as in Equation (2.69). Therefore, the formula for stress in a beam under simple bending cannot be used and Equation (7.3) is not valid. In case of a positive axial force Offpipe overestimates the bending strain. This is clearly noticeable when the second-order terms of the bending moment are large and the pipe's curvature is large. The second-order terms of the bending moment are large when the pipe's bending stiffness is low, thus when its diameter is small.

Results of example S2 are compared in Figure 7.7 and in Table 7.8. In Figure 7.7a it can be seen that the resulting deformed shapes are approximately equal. In Figure $7.7 \mathrm{~b}$ it can be seen that the bending strains resulting from the new pipe-lay model and Offpipe are virtually equal. The overestimation of the bending strains is insignificant, because the pipe's diameter is larger and its bending stiffness is higher than that of the pipe in example S1.

The axial strains resulting from the two models differ significantly; those resulting from the new model are larger on the stinger. In the new pipe-lay model the axial strains in the pipe on the stinger are higher due to plastic material behaviour. In 


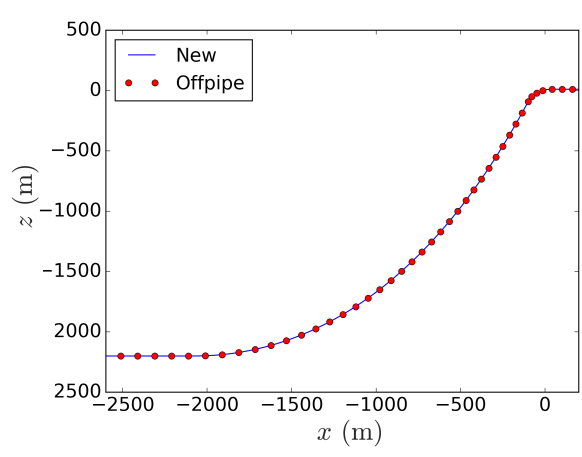

(a) Deformed shape of the pipe.

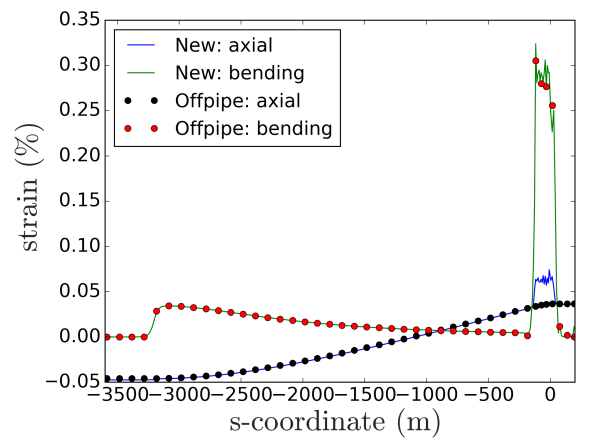

(b) Strains in the pipe.

Figure 7.7: Example S2.

Offpipe the effect of nonlinear material behaviour on the axial strain is not taken into account. It can be concluded that Offpipe's simplification leads to incorrect results when plastic deformation occurs.

Table 7.8: Results of static S-lay simulations compared with those of Offpipe.

\begin{tabular}{llllll}
\hline & \multicolumn{2}{c}{ Example S1 } & & \multicolumn{2}{c}{ Example S2 } \\
& New & Offpipe & & New & Offpipe \\
\cline { 2 - 3 } \cline { 5 - 6 } Top tension & $1288 \mathrm{kN}$ & $1280 \mathrm{kN}$ & & $7245 \mathrm{kN}$ & $7230 \mathrm{kN}$ \\
Departure angle & $84.2 \mathrm{deg}$ & $84.2 \mathrm{deg}$ & & $68.2 \mathrm{deg}$ & $68.2 \mathrm{deg}$ \\
Tip separation & $0.43 \mathrm{~m}$ & $0.43 \mathrm{~m}$ & & $0.90 \mathrm{~m}$ & $0.90 \mathrm{~m}$ \\
Maximum bending strain & $0.18 \%$ & $0.20 \%$ & & $0.32 \%$ & $0.31 \%$ \\
Maximum axial strain & $0.038 \%$ & $0.038 \%$ & & $0.054 \%$ & $0.037 \%$ \\
\hline
\end{tabular}

\subsubsection{Dynamic simulation}

In the new pipe-lay model, results of dynamic simulations are obtained in a similar way to those of the J-lay simulations. Properties of the numerical time integration are shown in Table 7.9. The initialisation procedure from Section 3.3 is used to damp high-frequency behaviour at the start of the simulation.

In dynamic simulations displacements of the tensioner and roller-boxes are prescribed by the response of the pipe-lay vessel to a generic wave, as described in Section 5.3.2. Properties of this wave are shown in Table 7.9. The resulting displacements of the stinger tip are shown in Figures 7.8a and 7.9a for examples $\mathrm{S} 1$ and S2 respectively.

The deformed shape of the pipe at time zero is determined from the result of the 
Table 7.9: Overview of S-lay examples.

\begin{tabular}{llll}
\hline & & Example S1 & Example S2 \\
\hline Time step & $\Delta t$ & $1 \mathrm{~s}$ & $1 \mathrm{~s}$ \\
Simulation time & $T_{\text {total }}$ & $600 \mathrm{~s}$ & $600 \mathrm{~s}$ \\
Initial period & $T_{\text {ini }}$ & $20 \mathrm{~s}$ & $20 \mathrm{~s}$ \\
Numerical damping & $\alpha$ & 0.03 & 0.03 \\
Water density & $\rho_{\mathrm{w}}$ & $1025 \mathrm{~kg} / \mathrm{m}^{3}$ & $1025 \mathrm{~kg} / \mathrm{m}^{3}$ \\
Significant wave height & $H_{\mathrm{s}}$ & $3 \mathrm{~m}$ & $4 \mathrm{~m}$ \\
Mean zero up-crossing period & $T_{\mathrm{z}}$ & $7 \mathrm{~s}$ & $9 \mathrm{~s}$ \\
\hline
\end{tabular}

corresponding static simulations of Section 7.2.1. In the result of the shape of the pipe, the node of the pipe's end that is in contact with the seabed is fixed in its place. At time zero the position of the stinger is not equal to its position in the static simulation. The position of the stinger is determined similar to that of the J-lay hinge in examples J1 and J2.

In Figure 7.8 and Table 7.10 results of example S1 are presented. In Figure 7.8b it can be seen that the maximum strains in the sagbend are smaller in the results from the new pipe-lay model. The sagbend is the section of the pipe between the stinger and the seabed. These smaller maximum strains can be explained by the simplified tensioner model, which continuously pays the pipe in and out. This overactive tensioner behaviour results in smaller displacements of the pipe in the sagbend and thus in smaller strains. When the tensioner model is disabled the pipe's displacements and the maximum strains are larger. In Offpipe, the tensioner pays the pipe in or out only when the tensioner limits are exceeded, which is a better approximation of reality. It can be concluded that the tensioner model described in Section 4.5 is insufficient.

Table 7.10: Results of dynamic S-lay simulations compared with those of Offpipe.

\begin{tabular}{llllll}
\hline & \multicolumn{2}{c}{ Example S1 } & & \multicolumn{2}{c}{ Example S2 } \\
& New & Offpipe & & New & Offpipe \\
\cline { 2 - 3 } \cline { 5 - 6 } Max top tension & $1517 \mathrm{kN}$ & $1476 \mathrm{kN}$ & & $8587 \mathrm{kN}$ & $8735 \mathrm{kN}$ \\
Min top tension & $1050 \mathrm{kN}$ & $1085 \mathrm{kN}$ & & $5513 \mathrm{kN}$ & $5746 \mathrm{kN}$ \\
Max tip separation & $0.77 \mathrm{~m}$ & $1.12 \mathrm{~m}$ & & $2.42 \mathrm{~m}$ & $3.10 \mathrm{~m}$ \\
Min tip separation & $0.21 \mathrm{~m}$ & $0.10 \mathrm{~m}$ & & $0.32 \mathrm{~m}$ & $0.11 \mathrm{~m}$ \\
Max bending strain & $0.21 \%$ & $0.22 \%$ & & $0.32 \%$ & $0.32 \%$ \\
Max axial strain & $0.045 \%$ & $0.045 \%$ & & $0.062 \%$ & $0.047 \%$ \\
\hline
\end{tabular}

In Figure 7.9 and Table 7.10 results of example $\mathrm{S} 2$ are presented. In Figure 7.9b it can be seen that the bending strains on the stinger resulting from the two models are similar. Their respective maximum bending strains are equal, as can be seen in Table 7.10. The maximum axial strain and bending strain in the sagbend are 

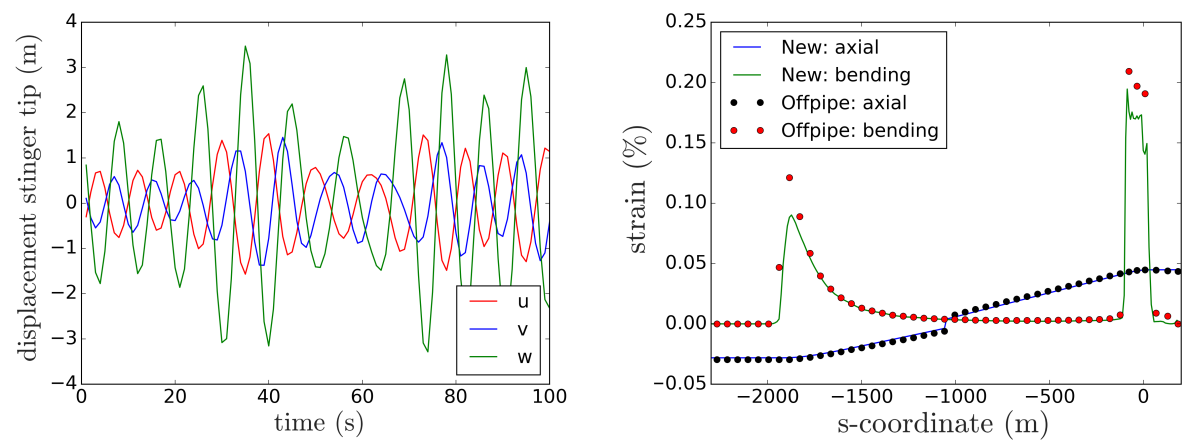

(a) Prescribed displacements of the stinger (b) Maximum axial and bending strains in tip. the pipe.

Figure 7.8: Example S1.

larger in the results from Offpipe, comparable with the results of example S1. On the stinger a difference in axial strain can be seen, similar to that in the static results of example $\mathrm{S} 2$.

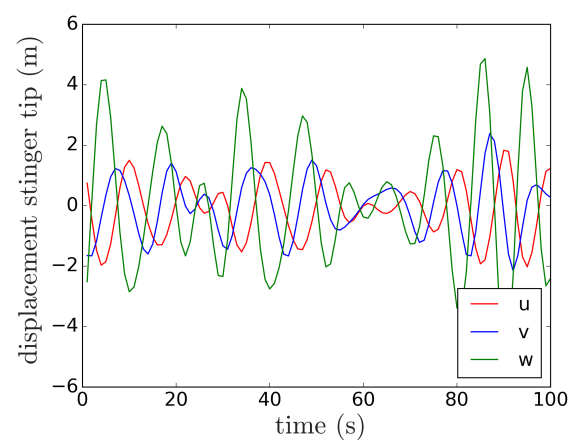

(a) Prescribed displacements of the stinger tip.

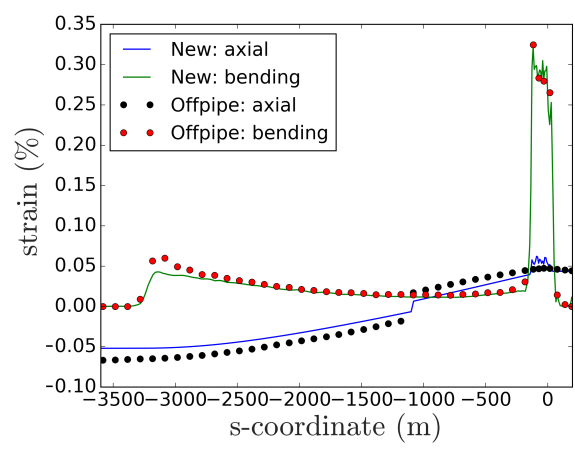

(b) Maximum axial and bending strains in the pipe.

Figure 7.9: Example S2.

In the new pipe-lay model the time step was set to $\Delta t=1 \mathrm{~s}$ for both S-lay examples. In Offpipe the time step has to be set to $\Delta t=0.1 \mathrm{~s}$ for example S1 and to $\Delta t=0.01 \mathrm{~s}$ for example S2. For both these examples larger time steps were not possible in Offpipe because of problems with convergence of the Newton-Raphson algorithm. It can be concluded that the new pipe-lay model is robust, while Offpipe shows convergence issues with large time steps.

In section 7.1 the time step sizes were equal and the computational time was 
approximately equal for both models. Because the time steps can be significantly smaller in the new pipe-lay model for S-lay simulations, it can be concluded that the new pipe-lay model is significantly more efficient.

\subsection{Start-up with a free pipe end}

In this section the results of a static simulation of a pipe-laying start-up with a free pipe end are presented. This example illustrates the effect of residual curvature on a free pipe end. Furthermore, this example shows a limitation of the submerged weight model.

In this start-up procedure the free pipe end is lowered to the seabed by paying the pipe out over the stinger. When the free end is near the seabed, it is hooked to a cable. The cable is used to apply an axial tension to the pipe, such that the characteristic S-shape is obtained.

In the simulation the deformed shape of the pipe is obtained by applying loads in three steps. In the first load step gravity and buoyancy loads are applied to the pipe. This results in a pipe that hangs perfectly vertical from the tip of the stinger. In the second load step a residual curvature is applied to all elements between the stinger and the free pipe end. In the third load step a horizontal tension is applied to the free pipe end to simulate the axial tension applied by the cable.

Residual curvature is defined by the plastic strain $\overline{\mathcal{E}}^{\mathrm{p}}$ from Equation (6.63) and can be seen as the curvature of the pipe after unloading. In this example the residual curvature that is set to all elements is equal to the maximum residual curvature that occurs in one of the elements in the first load step. This overestimates the residual curvature in the pipe sections near the seabed because these sections passed the stinger while subject to a lower axial tension. A constant residual curvature over the length of the pipe is sufficient for this illustrative example. The accuracy of the result can be increased by determining the residual curvature for each element separately.

The example is simulated twice, once using the detailed buoyancy model and once using the submerged weight model. The resulting shapes of the pipe after the second load step is shown in Figure 7.10. In this step the residual curvature is included but the horizontal load has not been applied yet. It can be seen that the pipe bends due to the residual curvature, such that the free pipe end moves to a position under the stinger.

In Figure 7.10 a difference between the two simulations can be seen. When the detailed buoyancy model is used instead of the submerged weight model, the pipe's curvature and the displacement of the free pipe end are larger. This difference is a result of the hydrostatic loads due to the pipe's curvature and due to the capped pipe end; these are included in the detailed buoyancy model, but disregarded in the submerged weight model. 


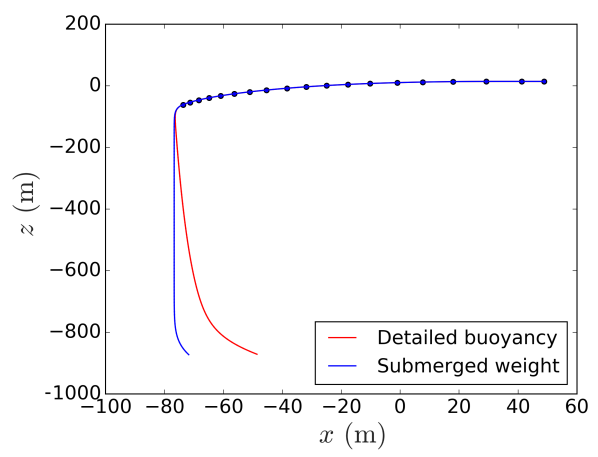

Figure 7.10: Shape of the pipe in free hanging start-up simulation.

When the free pipe end reaches the seabed, it is connected to a cable. This is simulated in the third load step by applying a horizontal load of $270 \mathrm{kN}$ to the free end of the pipe. The resulting shape of the pipe is shown in Figure 7.11a.

In this example the pipe rotates about its local $x_{\mathrm{L}}$ axis. The free pipe end rotates approximately 110 degrees about this axis, as can be seen in Figure 7.11b. In the model, this rotation is only obtained when a small force is applied in the $y$-direction.
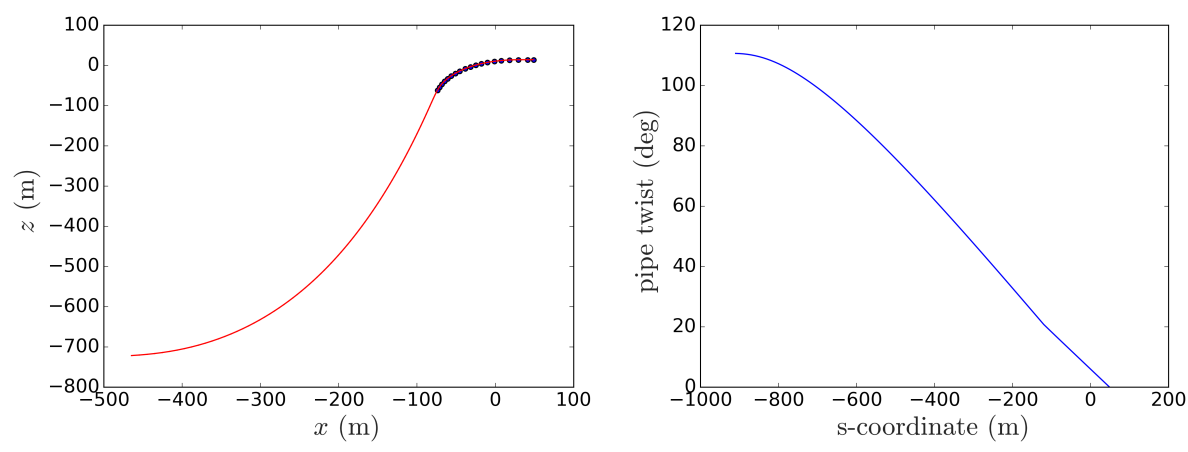

(a) Shape of the pipe after applying (b) Rotation about the local $x_{\mathrm{L}}$ axis. horizontal load.

Figure 7.11: Example S2.

It can be concluded that a simulation with a free pipe end requires the detailed buoyancy model. Additionally, pipe twist can be simulated with the new pipe-lay model. 


\subsection{Closure}

In this chapter results obtained with the new pipe-lay model are compared with results obtained with Offpipe. Two J-lay examples and two S-lay examples were analysed statically and dynamically. Additionally, an example of a pipe-lay startup with a free pipe end was presented.

The results of linear elastic J-lay simulations with the new pipe-lay model are approximately equal to those of Offpipe. Since the new pipe-lay model contains a detailed buoyancy model and Offpipe contains a submerged weight model, it can be concluded that the computationally inexpensive submerged weight model is sufficiently accurate for J-lay simulations with linear elastic material behaviour.

From the results of S-lay simulations it can be concluded that for pipes with a small diameter bending strains are overestimated in Offpipe. When plastic deformation occurs, Offpipe underestimates the axial strain.

From the results of dynamic S-lay simulations it can be concluded that the new pipe-lay model underestimates the maximum bending and axial strains in the sagbend. This is caused by the simplified tensioner model. For future research it is recommended that a more detailed tensioner model be implemented.

Computational costs for J-lay simulations were approximately equal for the new pipe-lay model and Offpipe. For these simulations the time step was equal. For the S-lay examples Offpipe requires time steps that are up to 100 times smaller than those for the new pipe-lay model. This makes the new pipe-lay model significantly more computationally efficient.

The new pipe-lay model can be used to simulate a pipe-lay start-up with a free pipe end. This is not possible in Offpipe because the detailed buoyancy model is required to obtain accurate results. Additionally, the new pipe-lay model can be used to assess pipe twist in S-lay simulations by setting a residual curvature to the pipe sections that have passed the stinger. 



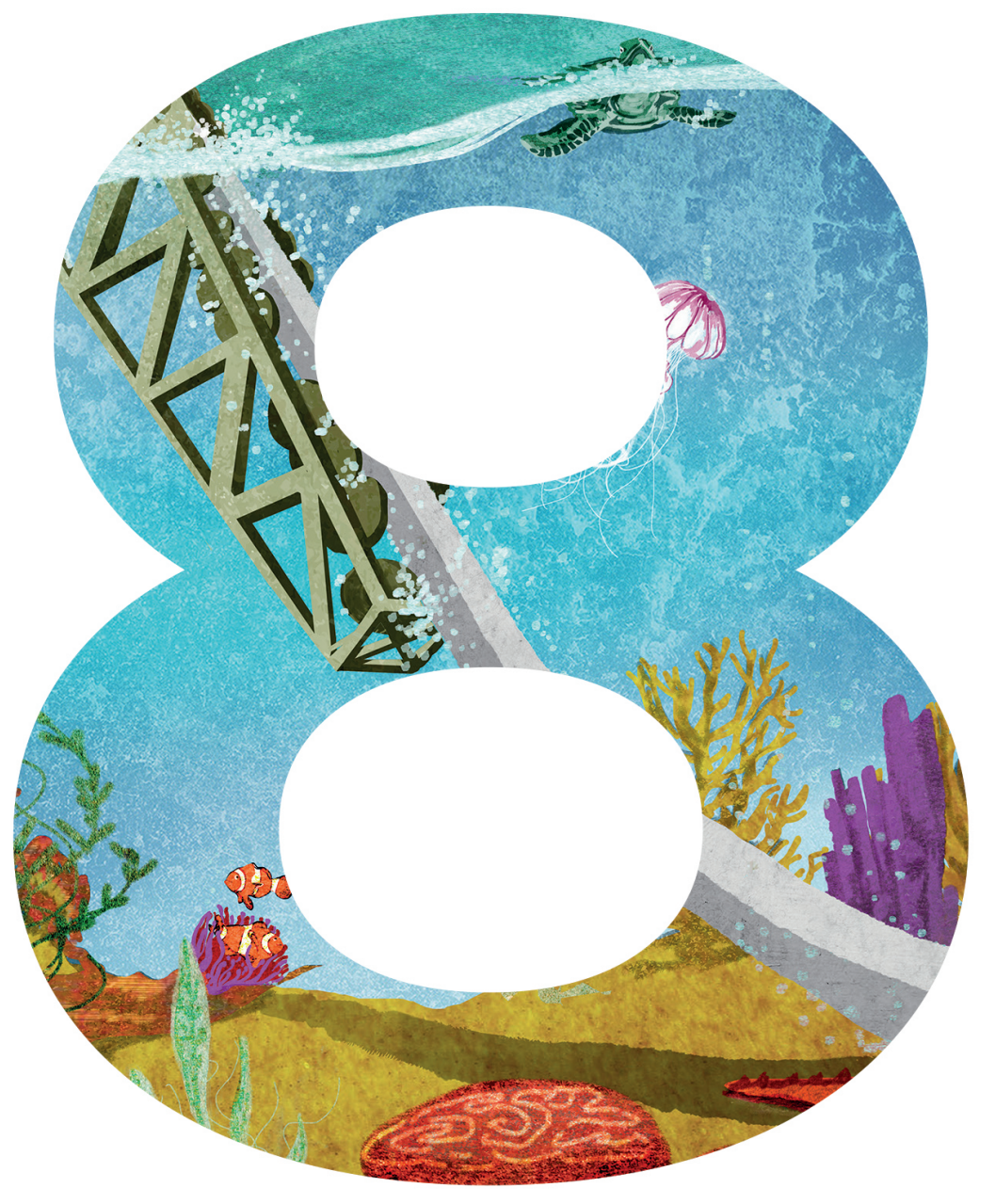




\section{8 \\ Conclusions and recommendations}

In this thesis an accurate numerical model for the simulation of offshore pipelaying has been developed with a focus on improving efficiency and accuracy in comparison with existing numerical models. The developed model can be used to assess the integrity of the pipe before executing the offshore laying process.

For the realisation of this numerical model a broad range of topics was studied in this work, varying from ship motions to plastic material behaviour. Combined, these topics form a complete model for the simulation of offshore pipe-laying as demonstrated in the examples of Chapter 7. The major conclusions of this research are summarised in Section 8.1 and recommendations for future improvements of the developed pipe-lay model are presented in Section 8.2.

\subsection{Conclusions}

\section{New geometric non-linear beam element}

In the pipe-lay model the pipe is modelled with beam finite elements. These elements can be subject to large displacements and rotations, resulting in geometric non-linear behaviour. A popular approach to modelling this nonlinearity is the corotational formulation, in which a local coordinate system moves and rotates with the element. In Chapter 2 the co-rotational beam element formulation of Crisfield is improved by changing the matrix that rotates the local element forces to the global coordinate system. Using the new rotation matrix, a new geometric stiffness matrix is derived. In comparison with the geometric stiffness matrix of the original formulation, this new matrix is computationally inexpensive. 
With the new rotation matrix nodal moments are rotated from the local coordinate system to the global coordinate system, such that each torsional and bending moment is in equilibrium with the corresponding moment in the adjacent element. The new formulation is able to predict rotations and displacements correctly when large torsional moments are involved. This is in contrast to the original formulation of Crisfield. The new formulation has similar or more accurate results and lower computational cost than the formulations of Crisfield and Battini-Pacoste, from which it can be concluded that the new formulation is the most efficient of the three.

\section{Initialisation procedure for numerical time integration}

In a numerical model the number of natural frequencies is equal to the number of degrees of freedom. High frequencies can occur that are physically not relevant and possibly numerically inaccurate. These high frequencies can be excited by a sudden load, which can occur at the start of a numerical simulation. The initialisation procedure for the HHT- $\alpha$ method, which is presented in Chapter 3, effectively damps these high frequencies at the start of a simulation.

\section{Contact-state procedure}

In an S-lay simulation nodes of the pipe can be in contact with the seabed and with roller-boxes on the stinger and on the vessel. This contact is modelled with the Penalty method, in which the contact state is binary: there is either 'contact' or 'no contact'.

In the 'global' iterative algorithm to determine force balance the contact-state of a node can alternate between 'contact' and 'no contact' over consecutive iterations, thereby hindering convergence. The delayed contact-state procedure that is described in Chapter 4 prevents this alternating behaviour.

\section{Detailed buoyancy model compared with submerged weight}

The submerged section of the pipe is subject to hydrostatic buoyancy forces. For a fully submerged body an 'upthrust' resulting from these hydrostatic forces can be determined with Archimedes' law. When buoyancy loads on a pipe element are modelled by Archimedes' law, the axial tension and strain must be corrected in a post-processing step.

This computationally inexpensive submerged weight model is sufficient for J-lay and S-lay simulations with linear elastic material behaviour. It is insufficient when local effects are involved that depend on the axial force or axial strain, such as 
plastic material behaviour and simulations with a free pipe end. The buoyancy method described in Chapter 5 should then be used instead.

\section{Dedicated plasticity algorithm}

The pipe stresses in radial direction are much smaller than the stresses in circumferential and axial direction, such that a plane stress state can be assumed. Stresses in circumferential direction are defined by the internal and external pressure acting on the pipe. The dedicated return mapping algorithm, which is derived based on these assumptions in Chapter 6, predicts stresses with arbitrary isotropic hardening that are identical to those predicted by a 3D return mapping algorithm. The dedicated return mapping algorithm is significantly more efficient.

In the pipe-lay model force balance is determined with a 'global' iterative algorithm. In the iteration process relatively large axial strains can occur that lead to an incorrect prediction of plastic deformations and bad convergence. The implementation of the dedicated return mapping algorithm in the co-rotational element, described in Chapter 6, prevents these incorrect predictions.

\section{New pipe-lay model compared with Offpipe}

Results from linear elastic J-lay simulations of the new pipe-lay model are approximately equal to those of Offpipe. These simulations include geometric nonlinearity, contact with the seabed, vessel motion and buoyancy.

Results from linear elastic S-lay simulations of the new pipe-lay model are different from those of Offpipe. From these results it is concluded that Offpipe overestimates bending strains for pipes with a small diameter that are subject to a positive axial load. This overestimation is caused by an improper calculation of the bending strains. In the new pipe-lay model bending strains are correctly calculated from the curvature of the pipe, resulting in lower bending strains than those resulting from Offpipe.

When plastic deformation occurs, Offpipe underestimates the axial strain. This underestimation is cause by a simplification in the calculation of the non-linear material behaviour. Offpipe assumes a non-linear relation between bending moment and curvature and disregards the effects of plastic material behaviour on the axial strain. In the new pipe-lay model plasticity is modelled properly, resulting in a higher axial strain than those resulting from Offpipe.

The new pipe-lay model can be used to simulate a start-up procedure with a free pipe end, which requires the detailed buoyancy model. The new pipe-lay model can also be used to model residual curvature in the pipe, which results from the plastic deformation on the stinger. These simulations are not possible in Offpipe. 
Computational costs for J-lay simulations are approximately equal for the new pipe-lay model and Offpipe. For these simulations the time step is equal in size. For S-lay simulations the new pipe-lay model is computationally significantly cheaper than Offpipe. The difference in computational costs can be attributed to the difference in maximum allowable time step size. In the new pipe-lay model numerical time integration can be applied with time steps that are up to 100 times larger than those in Offpipe. These larger time steps are possible due the algorithms described in this thesis, such as the contact-state procedure and the implemented plasticity model.

\subsection{Recommendations}

\section{Initialisation of static simulation}

To start the dynamic simulations the result of a static simulation is required. In the static simulation an initially horizontal, undeformed pipe is loaded with gravity and buoyancy loads. Several load steps are required for the 'global' iterative procedure to converge. Computational costs of the static simulation can be decreased by decreasing the number of load steps. When an initial guess for the shape of the pipe is used that is close to the actual solution, the number of load steps can be reduced to one.

In the overbend the displacements and rotations of the nodes are determined by the geometry of the stinger and the roller-boxes. The initial guess for the shape of the pipe can be resolved accordingly. In the sagbend the initial guess for the displacements and rotations of the nodes can be determined by an analytical approximation, such as the stiffened catenary method [40]. It is recommended to use this initial guess for the shape of the pipe instead of the initial horizontal pipe.

\section{Tensioner model}

The tensioner measures the axial tension and controls the tension by paying the pipe in or out. In the tensioner model these two functions are separated. This separation successfully ensures that the pipe nodes that are in contact with the roller-boxes remain in a correct position when the pipe moves axially.

Paying the pipe in and out is modelled as a continuous process. From the examples in Chapter 7 it is concluded that, with the current tensioner model, the strains in the sagbend are underestimated in the sagbend.

A better approximation of the tensioner behaviour can be obtained by a tensioner model that pays the pipe in or out only when the deadband is exceeded, instead of being a continuous process. A challenge in the development of this detailed model is to retain the large time step size, which should not be limited for efficiency. 
For future research it is recommended that a more detailed tensioner model be developed and implemented.

\section{Random sea state}

In the pipe-lay model generic surface waves are implemented as a superposition of regular waves with random shifts in phase angle. Each regular wave is determined for a certain frequency within the range of the wave spectrum. The resulting wave is periodic, thus it is not truly a random sea state.

It is recommended that the generic wave be modelled without periodicity within the maximum simulated time of three hours. A possible approach is to implement an equal area discretisation method, in which the wave spectrum is discretised into frequency intervals that have equal spectral energy.

\section{Validation of numerical results}

In this work results of the new pipe-lay model are compared with those of industry standard software Offpipe. From this comparison it was concluded that results of Offpipe can be inaccurate for certain simulations. Therefore, the comparison cannot be seen as a true validation of the new pipe-lay model. The comparison gives only an indication of the accuracy of the results when the same assumptions are made.

It is recommended that results of the new pipe-lay model be validated with results of other simulation software, such as Orcaflex [46] and Pipelay [65]. The results of the new pipe-lay model should preferably be validated with on-board measurements.

\section{Further reduction of computational costs}

Computational costs can be further reduced by efficient programming, such as parallel processing. The current pipe-lay model uses only one processor, while multiple are available on an average modern computer.

Parallel processing is often used to reduce computational costs of the solver for the linear system. In the pipe-lay model this system consists of a stiffness matrix that is relatively small and has a narrow band width. The system is solved with LUdecomposition implemented in FeaTure [61], which is very efficient for this simple matrix structure. Thus, a reduction in total calculation time is not expected when the computational time of the solver is further reduced.

In each iteration the assembly of the global stiffness matrix and force vector are the most computational expensive operations. This can be made more efficient by using parallel processing, which will have some overhead costs. Alternatively, 
parallel processing can be used to execute several pipe-lay simulations simultaneously. In practice, installation engineers often run several simulations, for example for different water depths and encountering wave angles. Running simultaneous simulations does not have overhead costs and is therefore expected to be the most efficient choice.

\section{On-board simulation tool}

The simulation time of the pipe-lay model is much smaller that the simulated time period. The model is a good basis for a real-time on-board simulation tool. Such a simulation tool can give a good insight into the integrity of the pipe during the laying process, in addition to the simulation results that are obtained before the laying process. It is recommended to investigate the implementation of the pipe-lay model in an on-board simulation and monitoring tool. 



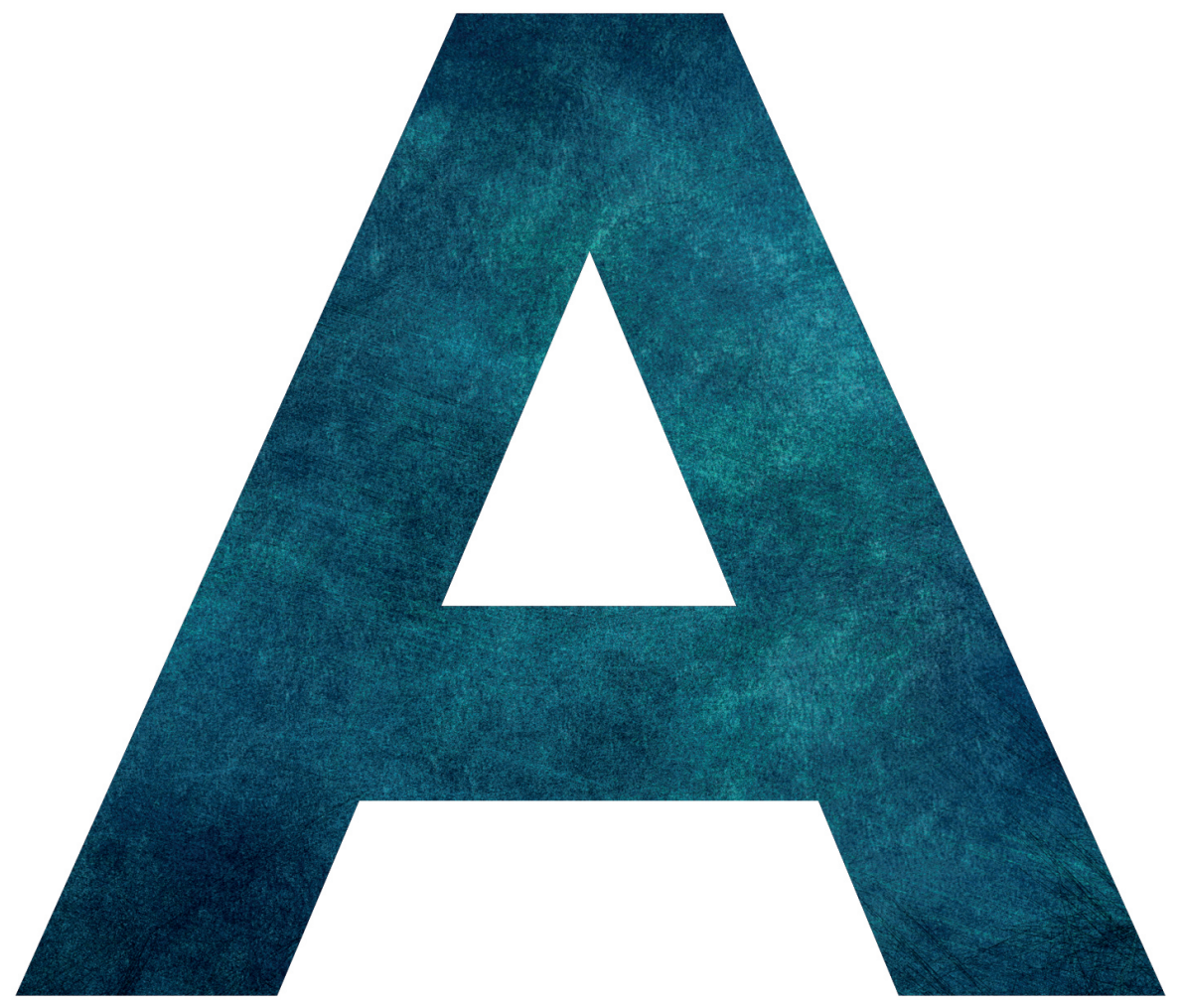




\section{Contributions from external loads to the stiffness matrix}

In this appendix the derivation of several contributions to the stiffness matrix of the co-rotational beam element is presented. The stiffness matrix is required for the Newton-Raphson algorithm, as presented in Section 2.3. By using a consistently derived stiffness matrix, quadratic convergence is achieved.

In the procedure that is applied in this appendix, a stiffness matrix $\mathbf{K}$ is derived by determining the variation of a force vector $\delta \boldsymbol{F}$ for a variation in the global degrees of freedom vector $\delta \boldsymbol{p}$ :

$$
\delta \boldsymbol{F}=\frac{\partial \boldsymbol{F}}{\partial \boldsymbol{p}} \delta \boldsymbol{p}=\mathbf{K} \delta \boldsymbol{p}
$$

At element level the global degrees of freedom vector $\boldsymbol{p}$ is given in Equation (2.6). The variation $\delta \boldsymbol{p}$ consist of the variations of translations and rotations:

$$
\delta \boldsymbol{p}=\left[\begin{array}{llll}
\delta \boldsymbol{d}_{1}^{\mathrm{T}} & \delta \Delta \boldsymbol{\alpha}_{1}^{\mathrm{T}} & \delta \boldsymbol{d}_{2}^{\mathrm{T}} & \delta \Delta \boldsymbol{\alpha}_{2}^{\mathrm{T}}
\end{array}\right]^{\mathrm{T}}
$$

In some derivations the variation of the difference in displacement between the first and the second node is used:

$$
\delta \boldsymbol{d}_{21}=\delta \boldsymbol{d}_{2}-\delta \boldsymbol{d}_{1}
$$

\section{A.1 Buoyancy}

The buoyancy loads are external forces in the static force balance and in the dynamic equation of motion. The buoyancy loads contribute to the stiffness matrix in three parts: distributed pressure, curvature effects and capped ends. 


\section{A.1.1 Distributed pressure}

Buoyancy forces and moments due to a distributed pressure on a straight element are given in Equation (5.11):

$$
\boldsymbol{F}_{1}^{\mathrm{b}}=\bar{q}_{\mathrm{b}}\left[\begin{array}{c}
b_{1} L \boldsymbol{e}_{\mathrm{n}} \\
b_{\mathrm{m} 1} L^{2}\left(\boldsymbol{e}_{1} \times \boldsymbol{e}_{\mathrm{n}}\right) \\
b_{2} L \boldsymbol{e}_{\mathrm{n}} \\
b_{\mathrm{m} 2} L^{2}\left(\boldsymbol{e}_{1} \times \boldsymbol{e}_{\mathrm{n}}\right)
\end{array}\right]
$$

To determine the variation of this force term, the variations of several sub-terms are derived. The variation $\delta L$ results in the fourth row of the transformation matrix, Equation (2.14), while the variation $\delta \boldsymbol{e}_{1}$ is known from Equation (2.16). The variation of $\bar{q}_{\mathrm{b}}$, Equation (5.3) is:

$$
\delta \bar{q}_{\mathrm{b}}=q_{\mathrm{b}} \delta \boldsymbol{e}_{\mathrm{n}}^{\mathrm{T}} \boldsymbol{k}
$$

Vector $\boldsymbol{e}_{\mathrm{n}}$ is given in Equation (5.4); its variation is determined to be:

$$
\delta \boldsymbol{e}_{\mathrm{n}}=\mathbf{Z} \delta \boldsymbol{d}_{21}
$$

with

$$
\mathbf{Z}=-\frac{\left(\boldsymbol{e}_{1} \boldsymbol{k}^{\mathrm{T}}+\boldsymbol{e}_{1}^{\mathrm{T}} \boldsymbol{k}\right) \mathbf{A}}{\left\|\boldsymbol{k}-\boldsymbol{e}_{1}^{T} \boldsymbol{k} \boldsymbol{e}_{1}\right\|}-\frac{\left(\boldsymbol{k}-\boldsymbol{e}_{1}^{T} \boldsymbol{k} \boldsymbol{e}_{1}\right)^{\mathrm{T}}\left(\boldsymbol{e}_{1} \boldsymbol{k}^{\mathrm{T}}+\boldsymbol{e}_{1}^{\mathrm{T}} \boldsymbol{k}\right) \mathbf{A}}{\left\|\boldsymbol{k}-\boldsymbol{e}_{1}^{T} \boldsymbol{k} \boldsymbol{e}_{1}\right\|^{3}}
$$

Here, $\mathbf{A}$ is the $3 \times 3$ matrix resulting from the variation of $\boldsymbol{e}_{1}$, as shown in Equation (2.16).

The factors $b_{1}$ and $b_{2}$ are shown in Equation (5.6); their variation is determined as functions of $\delta \bar{h}$ :

$$
\begin{aligned}
\delta b_{1} & =(1-\bar{h}) \delta \bar{h} \\
\delta b_{2} & =\bar{h} \delta \bar{h}
\end{aligned}
$$

$\bar{h}$ is shown in Equation (5.6); its variation is determined using the product rule:

$$
\delta \bar{h}=\frac{\bar{h}^{2}-\bar{h}}{h_{1}} \boldsymbol{k}^{\mathrm{T}} \delta \boldsymbol{d}_{1}-\frac{\bar{h}^{2}}{h_{1}} \boldsymbol{k}^{\mathrm{T}} \delta \boldsymbol{d}_{2}
$$

Using the equations for the variation of $b_{1}$ and $b_{2}$, the scalars $\lambda_{1}, \lambda_{12}$ and $\lambda_{2}$ are defined. $\lambda_{1}$ follows from terms with $\boldsymbol{k}^{\mathrm{T}} \delta \boldsymbol{d}_{1}$ in $\delta b_{1}, \lambda_{2}$ follows from terms with $\boldsymbol{k}^{\mathrm{T}} \delta \boldsymbol{d}_{2}$ in $\delta b_{2}$ and $\lambda_{12}$ follows from terms with $\boldsymbol{k}^{\mathrm{T}} \delta \boldsymbol{d}_{1}$ in $\delta b_{2}$. A scalar $\lambda_{12}$ can also be derived, resulting in $\lambda_{21}=\lambda_{12}$.

$$
\lambda_{1}=-(1-\bar{h})^{2} \frac{\bar{h}}{h_{1}} \quad \lambda_{2}=-\frac{\bar{h}^{3}}{h_{1}} \quad \lambda_{12}=-\frac{\bar{h}^{2}}{h_{1}}+\frac{\bar{h}^{3}}{h_{1}}
$$


The same procedure can be done for factors $b_{m 1}$ and $b_{m 2}$ from Equation (5.10). This yields the following scalars:

$$
\begin{array}{ll}
\lambda_{\mathrm{m} 1}=\left(\bar{h}^{3}-2 \bar{h}^{2}+\bar{h}\right) \frac{\bar{h}^{2}-\bar{h}}{h_{1}} & \lambda_{\mathrm{m} 2}=\left(\bar{h}^{3}-\bar{h}^{2}\right) \frac{-\bar{h}^{2}}{h_{1}} \\
\lambda_{\mathrm{m} 12}=\left(\bar{h}^{3}-2 \bar{h}^{2}+\bar{h}\right) \frac{-\bar{h}^{2}}{h_{1}} & \lambda_{\mathrm{m} 21}=-\lambda_{\mathrm{m} 12}
\end{array}
$$

With these variations the stiffness matrix resulting from the variation of Equation (5.11) is:

$$
\mathbf{K}_{1}^{\mathrm{b}}=q_{\mathrm{b}}\left[\begin{array}{cccc}
-b_{1} \mathbf{K}_{\mathrm{F}}+\lambda_{1} \mathbf{Q}_{\mathrm{F}} & \mathbf{0} & b_{1} \mathbf{K}_{\mathrm{F}}+\lambda_{12} \mathbf{Q}_{\mathrm{F}} & \mathbf{0} \\
-b_{\mathrm{m} 1} \mathbf{K}_{\mathrm{M}}+\lambda_{\mathrm{m} 1} \mathbf{Q}_{\mathrm{M}} & \mathbf{0} & b_{\mathrm{m} 1} \mathbf{K}_{\mathrm{M}}+\lambda_{\mathrm{m} 12} \mathbf{Q}_{\mathrm{M}} & \mathbf{0} \\
-b_{2} \mathbf{K}_{\mathrm{F}}+\lambda_{12} \mathbf{Q}_{\mathrm{F}} & \mathbf{0} & b_{2} \mathbf{K}_{\mathrm{F}}+\lambda_{2} \mathbf{Q}_{\mathrm{F}} & \mathbf{0} \\
-b_{\mathrm{m} 2} \mathbf{K}_{M}+\lambda_{\mathrm{m} 21} \mathbf{Q}_{\mathrm{M}} & \mathbf{0} & b_{\mathrm{m} 2} \mathbf{K}_{\mathrm{M}}+\lambda_{\mathrm{m} 2} \mathbf{Q}_{\mathrm{M}} & \mathbf{0}
\end{array}\right]
$$

Here, $\mathbf{K}_{\mathrm{F}}$ and $\mathbf{K}_{\mathrm{M}}$ are $3 \mathrm{x} 3$ matrices:

$$
\begin{aligned}
\mathbf{K}_{\mathrm{F}} & =\boldsymbol{e}_{\mathrm{n}}^{\mathrm{T}} \boldsymbol{k} \boldsymbol{e}_{\mathrm{n}} \boldsymbol{e}_{1}^{\mathrm{T}}+L \boldsymbol{e}_{\mathrm{n}} \boldsymbol{k}^{\mathrm{T}} \mathbf{Z}+L \boldsymbol{e}_{\mathrm{n}}^{\mathrm{T}} \boldsymbol{k} \mathbf{Z} \\
\mathbf{K}_{\mathrm{M}} & =L^{2}\left(\boldsymbol{e}_{1} \times \boldsymbol{e}_{\mathrm{n}}\right) \boldsymbol{k}^{\mathrm{T}} \mathbf{Z}+2 \boldsymbol{e}_{\mathrm{n}}^{\mathrm{T}} \boldsymbol{k}\left(\boldsymbol{e}_{1} \times \boldsymbol{e}_{\mathrm{n}}\right) L \boldsymbol{e}_{1}^{\mathrm{T}}-\boldsymbol{e}_{\mathrm{n}}^{\mathrm{T}} \boldsymbol{k} L^{2} \tilde{\boldsymbol{e}}_{\mathrm{n}} A+\boldsymbol{e}_{\mathrm{n}}^{\mathrm{T}} \boldsymbol{k} L^{2} \tilde{\boldsymbol{e}}_{1} \mathbf{Z}
\end{aligned}
$$

And $\mathbf{Q}_{\mathrm{F}}$ and $\mathbf{Q}_{\mathrm{M}}$ are also $3 \times 3$ matrices:

$$
\begin{aligned}
& \mathbf{Q}_{\mathrm{F}}=L \boldsymbol{e}_{\mathrm{n}}^{\mathrm{T}} \boldsymbol{k} \boldsymbol{e}_{\mathrm{n}} \boldsymbol{k}^{\mathrm{T}} \\
& \mathbf{Q}_{\mathrm{M}}=\boldsymbol{e}_{\mathrm{n}}^{\mathrm{T}} \boldsymbol{k} L^{2}\left(\boldsymbol{e}_{1} \times \boldsymbol{e}_{\mathrm{n}}\right) \boldsymbol{k}^{\mathrm{T}}
\end{aligned}
$$

\section{A.1.2 Curvature effects}

The buoyancy forces due to curvature effects are given in Equation (5.14):

$$
\boldsymbol{F}_{2}^{\mathrm{b}}=q_{b}\left[\begin{array}{c}
h_{1} \tan \theta_{1} \boldsymbol{e}_{3}-h_{1} \tan \phi_{1} \boldsymbol{e}_{2} \\
\mathbf{0}_{3 \times 1} \\
-h_{2} \tan \theta_{2} \boldsymbol{e}_{3}+h_{2} \tan \phi_{2} \boldsymbol{e}_{2} \\
\mathbf{0}_{3 \times 1}
\end{array}\right]
$$

The variation of the first term in Equation (5.14) is found using the product rule.

$$
\begin{aligned}
\delta\left(h_{1} \tan \theta_{1} \boldsymbol{e}_{3}\right) & =\delta h_{1} \tan \theta_{1} \boldsymbol{e}_{3}+h_{1} \delta \tan \theta_{1} \boldsymbol{e}_{3}+h_{1} \tan \theta_{1} \delta \boldsymbol{e}_{3} \\
& =-\tan \theta_{1} \boldsymbol{e}_{3} \boldsymbol{k}^{\mathrm{T}} \delta \boldsymbol{d}_{1}+\frac{h_{1} \boldsymbol{e}_{3}}{\cos ^{2} \theta_{1}} \delta \theta_{1}+h_{1} \tan \theta_{1} \mathbf{Q}_{3} \delta \boldsymbol{p}
\end{aligned}
$$

Here, $\mathbf{Q}_{2}$ and $\mathbf{Q}_{3}$ are matrices that follows from the variation $\delta \boldsymbol{e}_{i}$ as shown in Equation (2.17). The variation of the other three terms from Equation (5.14) are 
found in a similar way to the first term:

$$
\begin{aligned}
& \delta\left(-h_{1} \tan \phi_{1} \boldsymbol{e}_{2}\right)=\tan \phi_{1} \boldsymbol{e}_{2} \boldsymbol{k}^{\mathrm{T}} \delta \boldsymbol{d}_{1}-\frac{h_{1} \boldsymbol{e}_{2}}{\cos ^{2} \phi_{1}} \delta \phi_{1}-h_{1} \tan \phi_{1} \mathbf{Q}_{2} \delta \boldsymbol{p} \\
& \delta\left(-h_{2} \tan \theta_{2} \boldsymbol{e}_{3}\right)=\tan \theta_{2} \boldsymbol{e}_{3} \boldsymbol{k}^{\mathrm{T}} \delta \boldsymbol{d}_{2}-\frac{h_{2} \boldsymbol{e}_{3}}{\cos ^{2} \theta_{2}} \delta \theta_{2}-h_{2} \tan \theta_{2} \mathbf{Q}_{3} \delta \boldsymbol{p} \\
& \delta\left(h_{2} \tan \phi_{2} \boldsymbol{e}_{2}\right)=-\tan \phi_{2} \boldsymbol{e}_{2} \boldsymbol{k}^{\mathrm{T}} \delta \boldsymbol{d}_{2}+\frac{h_{2} \boldsymbol{e}_{2}}{\cos ^{2} \phi_{2}} \delta \phi_{2}+h_{2} \tan \phi_{2} \mathbf{Q}_{2} \delta \boldsymbol{p}
\end{aligned}
$$

The variations $\delta \theta_{1}, \delta \phi_{1}, \delta \theta_{2}$ and $\delta \phi_{2}$ result in the second, third, sixth and seventh row of the transformation matrix, that is presented in Section 2.1.2. Here, these rows are displayed by row-vectors $\boldsymbol{b}_{2}, \boldsymbol{b}_{3}, \boldsymbol{b}_{6}$ and $\boldsymbol{b}_{7}$. Substituting these terms in the equations above yields the buoyancy stiffness matrix due to curvature:

$$
\begin{aligned}
\mathbf{K}_{2}^{\mathrm{b}}=q_{\mathrm{b}}\left[\begin{array}{cccc}
-\tan \theta_{1} \boldsymbol{e}_{3} \boldsymbol{k}^{\mathrm{T}}+\tan \phi_{1} \boldsymbol{e}_{2} \boldsymbol{k}^{\mathrm{T}} & \mathbf{0} & \mathbf{0} & \mathbf{0} \\
\mathbf{0} & \mathbf{0} & \mathbf{0} & \mathbf{0} \\
\mathbf{0} & \mathbf{0} & \tan \theta_{2} \boldsymbol{e}_{3} \boldsymbol{k}^{\mathrm{T}}-\tan \phi_{2} \boldsymbol{e}_{2} \boldsymbol{k}^{\mathrm{T}} & \mathbf{0} \\
\mathbf{0} & \mathbf{0} & \mathbf{0} & \mathbf{0}
\end{array}\right] \\
+q_{\mathrm{b}}\left[\begin{array}{c}
\frac{h_{1} \boldsymbol{e}_{3} \boldsymbol{b}_{2}^{\mathrm{T}}}{\cos ^{2} \theta_{1}}+h_{1} \tan \theta_{1} \mathbf{Q}_{3}-\frac{h_{1} \boldsymbol{e}_{2} \boldsymbol{b}_{3}^{\mathrm{T}}}{\cos ^{2} \phi_{1}}-h_{1} \tan \phi_{1} \mathbf{Q}_{2} \\
\frac{h_{2} \boldsymbol{e}_{3} \boldsymbol{b}_{6}^{\mathrm{T}}}{\cos ^{2} \theta_{2}}-h_{2} \tan \theta_{2} \mathbf{Q}_{3}+\frac{h_{2} \boldsymbol{e}_{2} \boldsymbol{b}_{7}^{\mathrm{T}}}{\cos ^{2} \phi_{2}}+h_{2} \tan \phi_{2} \mathbf{Q}_{2} \\
\mathbf{0}_{3 \times 12}
\end{array}\right]
\end{aligned}
$$

\section{A.1.3 Capped ends}

The buoyancy forces due to capped ends are given in Equation (5.15):

$$
\boldsymbol{F}_{3}^{\mathrm{b}}=q_{b}\left[\begin{array}{c}
h_{1} \boldsymbol{e}_{1} \\
\mathbf{0}_{3 \times 1} \\
\mathbf{0}_{3 \times 1} \\
\mathbf{0}_{3 \times 1}
\end{array}\right] \quad \text { or } \quad \boldsymbol{F}_{3}^{\mathrm{b}}=q_{b}\left[\begin{array}{c}
\mathbf{0}_{3 \times 1} \\
\mathbf{0}_{3 \times 1} \\
-h_{2} \boldsymbol{e}_{1} \\
\mathbf{0}_{3 \times 1}
\end{array}\right]
$$

The stiffness matrix corresponding to these forces is determined by taking the variation of Equation (5.15), which results in:

$$
\mathbf{K}_{3}^{\mathrm{b}}=q_{\mathrm{b}}\left[\begin{array}{cccc}
-\boldsymbol{e}_{1} \boldsymbol{k}^{\mathrm{T}}-h_{1} \mathbf{A} & \mathbf{0} & h_{1} \mathbf{A} & \mathbf{0} \\
\mathbf{0} & \mathbf{0} & \mathbf{0} & \mathbf{0} \\
\mathbf{0} & \mathbf{0} & \mathbf{0} & \mathbf{0} \\
\mathbf{0} & \mathbf{0} & \mathbf{0} & \mathbf{0}
\end{array}\right]
$$

Or when the capped end is at the second node:

$$
\mathbf{K}_{3}^{\mathrm{b}}=q_{\mathrm{b}}\left[\begin{array}{cccc}
\mathbf{0} & \mathbf{0} & \mathbf{0} & \mathbf{0} \\
\mathbf{0} & \mathbf{0} & \mathbf{0} & \mathbf{0} \\
-h_{2} \mathbf{A} & \mathbf{0} & \boldsymbol{e}_{1} \boldsymbol{k}^{\mathrm{T}}-h_{2} \mathbf{A} & \mathbf{0} \\
\mathbf{0} & \mathbf{0} & \mathbf{0} & \mathbf{0}
\end{array}\right]
$$




\section{A.1.4 Correction on cross-sectional area}

The fourth buoyancy force is given in Equation (5.20):

$$
\boldsymbol{F}_{4}^{\mathrm{b}}=q_{\mathrm{b}}\left[\begin{array}{c}
h_{1}\left(\frac{1}{\boldsymbol{t}_{1}^{T} \boldsymbol{e}_{1}}-1\right) \boldsymbol{t}_{1} \\
\mathbf{0}_{3 \times 1} \\
-h_{2}\left(\frac{1}{\boldsymbol{u}_{1}^{T} \boldsymbol{e}_{1}}-1\right) \boldsymbol{u}_{1} \\
\mathbf{0}_{3 \times 1}
\end{array}\right]
$$

In this force vector, the minus one term can be omitted, as explained in Section 5.1.2. The variation $\delta \boldsymbol{t}_{1}$ and $\delta \boldsymbol{u}_{1}$ can be found in Equation (2.36), while the variation of the other terms are used earlier in this appendix. Using the product rule the stiffness contribution becomes:

$$
\mathbf{K}_{4}^{\mathrm{b}}=q_{\mathrm{b}}\left[\begin{array}{ccccc}
\frac{h_{1} \boldsymbol{t}_{1} \boldsymbol{t}_{1}^{\mathrm{T}} \mathbf{A}}{\left(\boldsymbol{t}_{1}^{\mathrm{T}} \boldsymbol{e}_{1}\right)^{2}}-\frac{\boldsymbol{t}_{1} \boldsymbol{k}^{\mathrm{T}}}{\boldsymbol{t}_{1}^{T} \boldsymbol{e}_{1}} & \frac{h_{1} \tilde{\boldsymbol{t}}_{1}}{\boldsymbol{t}_{1}^{T} \boldsymbol{e}_{1}}-\frac{\boldsymbol{e}_{1}^{\mathrm{T}} \tilde{\boldsymbol{t}}_{1}}{\left(\boldsymbol{t}_{1}^{\mathrm{T}} \boldsymbol{e}_{1}\right)^{2}} & \mathbf{0} & \mathbf{0} \\
\mathbf{0} & \mathbf{0} & \mathbf{0} & \mathbf{0} \\
\mathbf{0} & \mathbf{0} & -\frac{\boldsymbol{u}_{1} \boldsymbol{k}^{\mathrm{T}}}{\boldsymbol{u}_{1}^{T} \boldsymbol{e}_{1}}-\frac{h_{2} \boldsymbol{u}_{1} \boldsymbol{u}_{1}^{\mathrm{T}} \mathbf{A}}{\left(\boldsymbol{u}_{1}^{\mathrm{T}} \boldsymbol{e}_{1}\right)^{2}} & \frac{h_{2} \tilde{\boldsymbol{u}}_{1}}{\boldsymbol{u}_{1}^{T} \boldsymbol{e}_{1}}-\frac{\boldsymbol{e}_{1}^{\mathrm{T}} \tilde{\boldsymbol{u}}_{1}}{\left(\boldsymbol{u}_{1}^{\mathrm{T}} \boldsymbol{e}_{1}\right)^{2}} \\
\mathbf{0} & \mathbf{0} & \mathbf{0} & \mathbf{0}
\end{array}\right]
$$

\section{A.2 Hydrodynamic loads}

The hydrodynamic loads resulting from Morison's equation are external forces in the equation of motion. Morison's equation consists of three parts: loads due to drag, added mass and pressure gradient. Each part contributes to the stiffness matrix. Before these three parts are derived, the variation of the water velocities and accelerations are determined.

\section{A.2.1 Water velocities}

The velocities of the water are given in Equation (5.28) for a regular wave:

$$
\dot{\boldsymbol{d}}_{\mathrm{w}}=\left[\begin{array}{c}
\cos \varphi \frac{h}{2} \omega e^{k z} \cos (\omega t-k \bar{x}) \\
\sin \varphi \frac{h}{2} \omega e^{k z} \cos (\omega t-k \bar{x}) \\
-\frac{h}{2} \omega e^{k z} \sin (\omega t-k \bar{x})
\end{array}\right]
$$

The variation of the water velocity in $x$-direction is calculated as:

$$
\begin{aligned}
\delta \dot{d}_{\mathrm{w}, x} & =\cos \varphi \frac{h k}{2} \omega e^{k z} \cos (\omega t-k \bar{x}) \delta z \\
& +\cos \varphi \frac{h k}{2} \omega e^{k z} \sin (\omega t-k \bar{x})(\cos \varphi \delta x+\sin \varphi \delta y)
\end{aligned}
$$


The same can be done for the variation of the water velocity in $y$ and $z$-direction:

$$
\delta \dot{\boldsymbol{d}}_{\mathrm{w}}=C\left[\begin{array}{ccc}
\cos ^{2} \varphi \sin \Theta & \cos \varphi \sin \varphi \sin \Theta & \cos \varphi \cos \Theta \\
\cos \varphi \sin \varphi \sin \Theta & \sin ^{2} \varphi \sin \Theta & \sin \varphi \cos \Theta \\
\cos \varphi \cos \Theta & \sin \varphi \cos \Theta & -\sin \Theta
\end{array}\right] \delta \boldsymbol{d}_{i}=\mathbf{G}_{i} \delta \boldsymbol{d}_{i}
$$

Here, subscript $i$ refers to the first or the second node of an element. $C$ and $\Theta$ are scalars:

$$
\begin{gathered}
C=\frac{1}{2} h k \omega e^{k z} \\
\Theta=\omega t-k \bar{x}
\end{gathered}
$$

\section{A.2.2 Water accelerations}

The accelerations of the water are given in Equation (5.28) for a regular wave:

$$
\ddot{\boldsymbol{d}}_{\mathrm{w}}=\left[\begin{array}{c}
-\cos \varphi \frac{h}{2} \omega^{2} e^{k z} \sin (\omega t-k \bar{x}) \\
-\sin \varphi \frac{h}{2} \omega^{2} e^{k z} \sin (\omega t-k \bar{x}) \\
-\frac{h}{2} \omega^{2} e^{k z} \cos (\omega t-k \bar{x})
\end{array}\right]
$$

The variation of the water acceleration in $x$-direction is calculated as:

$$
\begin{aligned}
\delta \ddot{d}_{\mathrm{w}, x} & =-\cos \varphi \frac{h k}{2} \omega^{2} e^{k z} \sin (\omega t-k \bar{x}) \delta z \\
& +\cos \varphi \frac{h k}{2} \omega^{2} e^{k z} \cos (\omega t-k \bar{x})(\delta x \cos \varphi+\delta y \sin \varphi)
\end{aligned}
$$

The same can be done for the variation of the acceleration in $y$ - and $z$-direction:

$$
\delta \ddot{\boldsymbol{d}}_{\mathrm{w}}=C \omega\left[\begin{array}{ccc}
\cos ^{2} \varphi \cos \Theta & \cos \varphi \sin \varphi \cos \Theta & -\cos \varphi \sin \Theta \\
\cos \varphi \sin \varphi \cos \Theta & \sin ^{2} \varphi \cos \Theta & -\sin \varphi \sin \Theta \\
-\cos \varphi \sin \Theta & -\sin \varphi \sin \Theta & -\cos \Theta
\end{array}\right] \delta \boldsymbol{d}_{i}=\mathbf{H}_{i} \delta \boldsymbol{d}_{i}
$$

Where $C$ and $\Theta$ are scalars given in Equation (A.27).

\section{A.2.3 Drag force}

In Equation (5.22) the drag force is given by:

$$
\boldsymbol{F}^{\mathrm{drag}}=\frac{1}{4} L_{0} \rho_{\mathrm{w}} C_{\mathrm{d}} D \dot{\boldsymbol{d}}_{\mathrm{rn}}\left\|\dot{\boldsymbol{d}}_{\mathrm{rn}}\right\|
$$

The variation of the drag force is calculated as:

$$
\delta \boldsymbol{F}^{\mathrm{drag}}=\frac{1}{2} L_{0} \rho C_{\mathrm{d}} D\left\|\dot{\boldsymbol{d}}_{\mathrm{rn}}\right\| \delta \dot{\boldsymbol{d}}_{\mathrm{rn}}
$$


The variation of the relative velocity is:

$$
\delta \dot{\boldsymbol{d}}_{\mathrm{r}}=\delta \dot{\boldsymbol{d}}_{\mathrm{w}}-\delta \dot{\boldsymbol{d}}
$$

The variation of the velocity of the pipe can be found from Equation (3.3):

$$
\delta \dot{\boldsymbol{d}}=a_{1} \delta \boldsymbol{d}
$$

The variation of the water velocity is given in Equation (A.26). The variation of the relative normal velocity is determined by the variation of Equation (5.24). Substituting Equation (A.33) and (A.34) yields:

$$
\begin{aligned}
\delta \dot{\boldsymbol{d}}_{\mathrm{rn}} & =\delta \dot{\boldsymbol{d}}_{\mathrm{r}}-\delta \dot{\boldsymbol{d}}_{\mathrm{r}}^{\mathrm{T}} \boldsymbol{e}_{1} \boldsymbol{e}_{1}-\dot{\boldsymbol{d}}_{\mathrm{r}}^{\mathrm{T}} \delta \boldsymbol{e}_{1} \boldsymbol{e}_{1}-\dot{\boldsymbol{d}}_{\mathrm{r}}^{\mathrm{T}} \boldsymbol{e}_{1} \delta \boldsymbol{e}_{1} \\
& =\left(\mathbf{I}-\boldsymbol{e}_{1} \boldsymbol{e}_{1}^{\mathrm{T}}\right)\left(\mathbf{G}_{i}-a_{1} \mathbf{I}\right) \delta \boldsymbol{d}_{i}-\left(\boldsymbol{e}_{1} \dot{\boldsymbol{d}}_{\mathrm{r}}^{\mathrm{T}}+\dot{\boldsymbol{d}}_{\mathrm{r}}^{\mathrm{T}} \boldsymbol{e}_{1} \mathbf{I}\right) \mathbf{A} \delta \boldsymbol{d}_{21}
\end{aligned}
$$

Substituting the variation of the relative normal velocity into Equation (A.32) yields the tangential stiffness matrix due to the drag force.

$$
\mathbf{K}^{\text {drag }}=\frac{1}{2} L_{0} \rho C_{\mathrm{d}} D\left\|\dot{\boldsymbol{d}}_{\mathrm{rn}}\right\|\left[\begin{array}{cccc}
\mathbf{K}_{\mathrm{a}} & \mathbf{0} & \mathbf{K}_{\mathrm{b}} & \mathbf{0} \\
\mathbf{0} & \mathbf{0} & \mathbf{0} & \mathbf{0} \\
\mathbf{K}_{\mathrm{c}} & \mathbf{0} & \mathbf{K}_{\mathrm{d}} & \mathbf{0} \\
\mathbf{0} & \mathbf{0} & \mathbf{0} & \mathbf{0}
\end{array}\right]
$$

With sub-matrices $\mathbf{K}_{\mathrm{a}}$ to $\mathbf{K}_{\mathrm{d}}$ :

$$
\begin{aligned}
& \mathbf{K}_{\mathrm{a}}=\left(\mathbf{I}-\boldsymbol{e}_{1} \boldsymbol{e}_{1}^{\mathrm{T}}\right)\left(\mathbf{G}_{1}-a_{1} \mathbf{I}\right)+\left(\boldsymbol{e}_{1} \dot{\boldsymbol{d}}_{\mathrm{r} 1}^{\mathrm{T}}+\dot{\boldsymbol{d}}_{\mathrm{r} 1}^{\mathrm{T}} \boldsymbol{e}_{1} \mathbf{I}\right) \mathbf{A} \\
& \mathbf{K}_{\mathrm{b}}=-\left(\boldsymbol{e}_{1} \dot{\boldsymbol{d}}_{\mathrm{r} 1}^{\mathrm{T}}+\dot{\boldsymbol{d}}_{\mathrm{r} 1}^{\mathrm{T}} \boldsymbol{e}_{1} \mathbf{I}\right) \mathbf{A} \\
& \mathbf{K}_{\mathrm{c}}=\left(\boldsymbol{e}_{1} \dot{\boldsymbol{d}}_{\mathrm{r} 2}^{\mathrm{T}}+\dot{\boldsymbol{d}}_{\mathrm{r} 2}^{\mathrm{T}} \boldsymbol{e}_{1} \mathbf{I}\right) \mathbf{A} \\
& \mathbf{K}_{\mathrm{d}}=\left(\mathbf{I}-\boldsymbol{e}_{1} \boldsymbol{e}_{1}^{\mathrm{T}}\right)\left(\mathbf{G}_{2}-a_{1} \mathbf{I}\right)-\left(\boldsymbol{e}_{1} \dot{\boldsymbol{d}}_{\mathrm{r} 2}^{\mathrm{T}}+\dot{\boldsymbol{d}}_{\mathrm{r} 2}^{\mathrm{T}} \boldsymbol{e}_{1} \mathbf{I}\right) \mathbf{A}
\end{aligned}
$$

Here, $\mathbf{I}$ is the identity matrix of size $3 \times 3$ and $\mathbf{G}_{i}$ is the $3 \times 3$ matrix from Equation (A.26).

\section{A.2.4 Added mass}

In Equation (5.22) the added mass term is given by:

$$
\boldsymbol{F}^{\text {addedmass }}=\frac{1}{8} L_{0} \rho_{\mathrm{w}} C_{\mathrm{a}} \pi D^{2} \ddot{\boldsymbol{d}}_{\mathrm{rn}}
$$

The variation of the added mass term is:

$$
\delta \boldsymbol{F}^{\text {addedmass }}=\frac{1}{8} L_{0} \rho_{\mathrm{w}} C_{\mathrm{a}} \pi D^{2} \delta \ddot{\boldsymbol{d}}_{\mathrm{rn}}
$$


The procedure to find the variation of the relative normal acceleration is similar to the procedure to find the variation of the relative normal velocity, which results in:

$$
\mathbf{K}^{\text {addedmass }}=\frac{1}{8} L_{0} \rho_{\mathrm{w}} C_{\mathrm{a}} \pi D^{2}\left[\begin{array}{cccc}
\mathbf{K}_{\mathrm{e}} & \mathbf{0} & \mathbf{K}_{\mathrm{f}} & \mathbf{0} \\
\mathbf{0} & \mathbf{0} & \mathbf{0} & \mathbf{0} \\
\mathbf{K}_{\mathrm{g}} & \mathbf{0} & \mathbf{K}_{\mathrm{h}} & \mathbf{0} \\
\mathbf{0} & \mathbf{0} & \mathbf{0} & \mathbf{0}
\end{array}\right]
$$

With sub-matrices $\mathbf{K}_{\mathrm{e}}$ to $\mathbf{K}_{\mathrm{h}}$ :

$$
\begin{aligned}
& \mathbf{K}_{\mathrm{a}}=\left(\mathbf{I}-\boldsymbol{e}_{1} \boldsymbol{e}_{1}^{\mathrm{T}}\right)\left(\mathbf{H}_{1}-a_{0} \mathbf{I}\right)+\left(\boldsymbol{e}_{1} \ddot{\boldsymbol{d}}_{\mathrm{r} 1}^{\mathrm{T}}+\ddot{\boldsymbol{d}}_{\mathrm{r} 1}^{\mathrm{T}} \boldsymbol{e}_{1} \mathbf{I}\right) \mathbf{A} \\
& \mathbf{K}_{\mathrm{b}}=-\left(\boldsymbol{e}_{1} \ddot{\boldsymbol{d}}_{\mathrm{r} 1}^{\mathrm{T}}+\ddot{\boldsymbol{d}}_{\mathrm{r} 1}^{\mathrm{T}} \boldsymbol{e}_{1} \mathbf{I}\right) \mathbf{A} \\
& \mathbf{K}_{\mathrm{c}}=\left(\boldsymbol{e}_{1} \ddot{\boldsymbol{d}}_{\mathrm{r} 2}^{\mathrm{T}}+\ddot{\boldsymbol{d}}_{\mathrm{r} 2}^{\mathrm{T}} \boldsymbol{e}_{1} \mathbf{I}\right) \mathbf{A} \\
& \mathbf{K}_{\mathrm{d}}=\left(\mathbf{I}-\boldsymbol{e}_{1} \boldsymbol{e}_{1}^{\mathrm{T}}\right)\left(\mathbf{H}_{2}-a_{0} \mathbf{I}\right)-\left(\boldsymbol{e}_{1} \ddot{\boldsymbol{d}}_{\mathrm{r} 2}^{\mathrm{T}}+\ddot{\boldsymbol{d}}_{\mathrm{r} 2}^{\mathrm{T}} \boldsymbol{e}_{1} \mathbf{I}\right) \mathbf{A}
\end{aligned}
$$

Here, $\mathbf{H}_{i}$ is the $3 \times 3$ matrix from Equation (A.30).

\section{A.2.5 Pressure gradient}

In Equation (5.22) the pressure gradient term is given by:

$$
\boldsymbol{F}^{\text {pressuregradient }}=\frac{1}{8} L_{0} \rho_{\mathrm{w}} \pi D^{2} \ddot{\boldsymbol{d}}_{\mathrm{wn}}
$$

Th variation of the pressure gradient term is:

$$
\delta \boldsymbol{F}^{\text {pressuregradient }}=\frac{1}{8} L_{0} \rho_{\mathrm{w}} \pi D^{2} \delta \ddot{\boldsymbol{d}}_{\mathrm{wn}}
$$

The variation of the water acceleration is found in Equation (A.30). The stiffness matrix due to the pressure gradient is:

$$
\mathbf{K}^{\text {pressuregradient }}=\frac{1}{8} L_{0} \rho_{\mathrm{w}} \pi D^{2}\left[\begin{array}{cccc}
\mathbf{K}_{\mathrm{i}} & \mathbf{0} & \mathbf{K}_{\mathrm{j}} & \mathbf{0} \\
\mathbf{0} & \mathbf{0} & \mathbf{0} & \mathbf{0} \\
\mathbf{K}_{\mathrm{k}} & \mathbf{0} & \mathbf{K}_{\mathrm{l}} & \mathbf{0} \\
\mathbf{0} & \mathbf{0} & \mathbf{0} & \mathbf{0}
\end{array}\right]
$$

With sub-matrices $\mathbf{K}_{\mathrm{i}}$ to $\mathbf{K}_{\mathrm{l}}$ : 


$$
\begin{aligned}
& \mathbf{K}_{\mathrm{i}}=\left(\mathbf{I}-\boldsymbol{e}_{1} \boldsymbol{e}_{1}^{\mathrm{T}}\right) \mathbf{H}_{1}+\left(\boldsymbol{e}_{1} \ddot{\boldsymbol{d}}_{\mathrm{w} 1}^{\mathrm{T}}+\ddot{\boldsymbol{d}}_{\mathrm{w} 1}^{\mathrm{T}} \boldsymbol{e}_{1}\right) \mathbf{A} \\
& \mathbf{K}_{\mathrm{j}}=-\left(\boldsymbol{e}_{1} \ddot{\boldsymbol{d}}_{\mathrm{w} 1}^{\mathrm{T}}+\ddot{\boldsymbol{d}}_{\mathrm{w} 1}^{\mathrm{T}} \boldsymbol{e}_{1}\right) \mathbf{A} \\
& \mathbf{K}_{\mathrm{k}}=\left(\boldsymbol{e}_{1} \ddot{\boldsymbol{d}}_{\mathrm{w} 2}^{\mathrm{T}}+\ddot{\boldsymbol{d}}_{\mathrm{w} 2}^{\mathrm{T}} \boldsymbol{e}_{1}\right) \mathbf{A} \\
& \mathbf{K}_{\mathrm{l}}=\left(\mathbf{I}-\boldsymbol{e}_{1} \boldsymbol{e}_{1}^{\mathrm{T}}\right) \mathbf{H}_{2}-\left(\boldsymbol{e}_{1} \ddot{\boldsymbol{d}}_{\mathrm{w} 2}^{\mathrm{T}}+\ddot{\boldsymbol{d}}_{\mathrm{w} 2}^{\mathrm{T}} \boldsymbol{e}_{1}\right) \mathbf{A}
\end{aligned}
$$




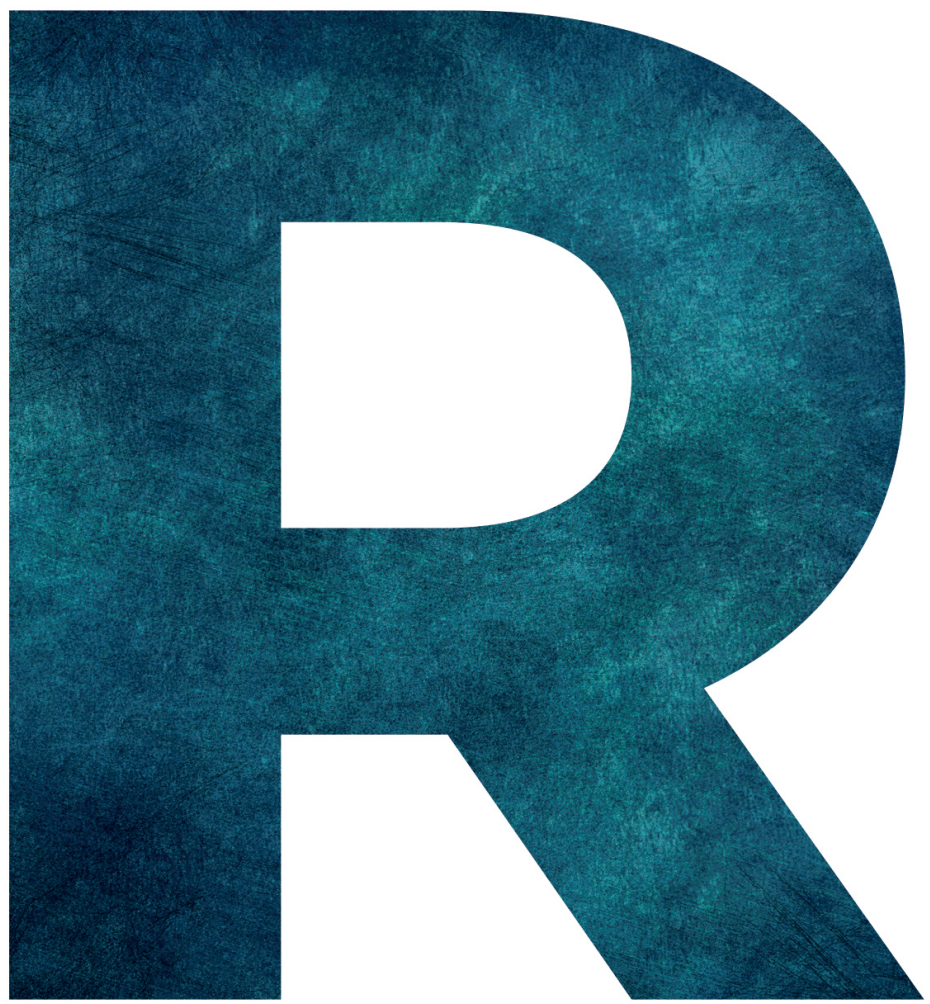




\section{References}

[1] M. Abramowitz and I. A. Stegun. Handbook of mathematical functions. Dover Publications, 9th edition, 1970.

[2] Allseas Engineering. Allseas Group S.A. https://allseas.com/. Accessed on 12-12-2019.

[3] N. D. P. Barltrop and A. J. Adams. Dynamics of Fixed Marine Structures. Butterworth-Heinemann, 3th edition, 1991.

[4] K.-J. Bathe and M. M. I. Baig. On a composite implicit time integration procedure for nonlinear dynamics. Computers $\&$ Structures, 83(31-32):25132524, Dec 2005.

[5] J.-M. Battini. Co-rotational beam elements in instability problems. $\mathrm{PhD}$ thesis, KTH Royal Institute of Technology, 2002.

[6] J.-M. Battini and C. Pacoste. Co-rotational beam elements with warping effects in instability problems. Computer Methods in Applied Mechanics and Engineering, 191(17-18):1755-1789, 2002.

[7] T. Belytschko and B. J. Hsieh. Non-linear transient finite element analysis with convected co-ordinates. International Journal for Numerical Methods in Engineering, 7(3):255-271, 1973.

[8] BP. BP Energy Outlook, 2019.

[9] C. L. Bretschneider. Wave variability and wave spectra for wind-generated gravity waves. U.S. Beach erosion board technical memorandum 118, 1959.

[10] J. Chung and G. M. Hulbert. A time integration algorithm for structural dynamics with improved numerical dissipation: The Generalized- $\alpha$ method. Journal of Applied Mechanics, 60(2):371-375, 1993.

[11] R. D. Cook. Concepts and applications of finite element analysis. John Wiley \& Sons, 4th edition, 2002. 
[12] J. W. Cooley and J. W. Tukey. An algorithm for the machine calculation of complex Fourier series. Mathematics of Computation, 19(90):297-301, 1965.

[13] M. A. Crisfield. A consistent co-rotational formulation for non-linear, threedimensional, beam-elements. Computer Methods in Applied Mechanics and Engineering, 81(2):131-150, 1990.

[14] M. A. Crisfield. Non-linear finite element analysis of solids and structures, Volume 1. John Wiley \& Sons, 1991.

[15] M. A. Crisfield. Non-linear finite element analysis of solids and structures, Volume 2. John Wiley \& Sons, 1997.

[16] R. de Borst. Zero-normal-stress condition in plane-stress and shell elastoplasticity. Communications in Applied Numerical Methods, 7(1):29-33, 1991.

[17] F. H. de Vries, H. J. M. Geijselaers, A. H. van den Boogaard, and A. Huisman. A nonlinear dynamic corotational finite element model for submerged pipes. In IOP Conference Series: Materials Science and Engineering, volume 276, 2017.

[18] F. H. de Vries, H. J. M. Geijselaers, A. H. van den Boogaard, and A. Huisman. Finite element modeling of pipe-laying dynamics using corotational elements. International Journal for Computational Methods in Engineering Science and Mechanics, 20(4):293-307, 2019.

[19] R. G. Dean and R. A. Dalrymple. Water wave mechanics for engineers and scientists (Vol. 2). World Scientific Publishing Co Inc, 1991.

[20] DNV GL. DNVGL-ST-F101: Submarine pipeline systems, 2017.

[21] M. A. Dokainish and K. Subbaraj. A survey of direct time-integration methods in computational structural dynamics-I. Explicit methods. Computers \& Structures, 32(6):1371-1386, Jan 1989.

[22] O. M. Faltinsen. Sea loads on ships and offshore structures. Cambridge University Press, 1993.

[23] C. A. Felippa and B. Haugen. A unified formulation of small-strain corotational finite elements: I. Theory. Computer Methods in Applied Mechanics and Engineering, 194(21-24 SPEC. ISS.):2285-2335, 2005.

[24] G. L. Goudreau and R. L. Taylor. Evaluation of numerical integration methods in elastodynamics. Computer Methods in Applied Mechanics and Engineering, 2(1):69-97, 1973.

[25] B. Guo, S. Song, J. Chacko, and A. Ghalambor. Offshore Pipelines. Elsevier, 2005. 
[26] K. Hasselmann, T. P. Barnett, E. Bouws, H. Carlson, D. E. Cartwright, K. Enke, J. A. Ewing, H. Gienapp, D. E. Hasselmann, P. Kruseman, A. Meerburg, and P. Muller. Measurements of wind-wave growth and swell decay during the Joint North Sea Wave Project (JONSWAP). Deutsche Hydrographischen Zeitschrift, 8(12), 1973.

[27] R. C. Hibbeler. Mechanics of Materials, SI Second Edition. Pearson Prentice Hall, Singapore, 2005.

[28] H. M. Hilber and T. J. R. Hughes. Collocation, dissipation and overshoot for time integration schemes in structural dynamics. Earthquake Engineering 83 Structural Dynamics, 6(1):99-117, 1978.

[29] H. M. Hilber, T. J. R. Hughes, and R. L. Taylor. Improved numerical dissipation for time integration algorithms in structural dynamics. Earthquake Engineering \&3 Structural Dynamics, 5(3):283-292, 1977.

[30] E. Hinton, T. Rock, and O. C. Zienkiewicz. A note on mass lumping and related processes in the finite element method. Earthquake Engineering \&6 Structural Dynamics, 4(3):245-249, 1976.

[31] J. C. Houbolt. A recurrence matrix solution for the dynamic response of elastic aircraft. Journal of the Aeronautical Sciences, 17(9):540-550, 1950.

[32] A. Ibrahimbegović. On the choice of finite rotation parameters. Computer Methods in Applied Mechanics and Engineering, 149(1-4):49-71, 1997.

[33] A. Ibrahimbegović, F. Frey, and I. Kožar. Computational aspects of vector-like parametrization of three-dimensional finite rotations. International Journal for Numerical Methods in Engineering, 38(21):3653-3673, 1995.

[34] G. Kloosterman. Contact methods in finite element simulations. PhD thesis, Universiteit Twente, 2002.

[35] R. C. Malahy. A nonlinear finite element method for the analysis of the offshore pipelaying problem. $\mathrm{PhD}$ thesis, Rice University, Houston, Texas, 1985.

[36] R. C. Malahy. Offpipe user's guide, 1996.

[37] MATLAB. version 9.3.0 (R2017b). The MathWorks Inc., Natick, Massachusetts, 2017.

[38] L. Meirovitch. Fundamentals of vibrations. McGraw-Hill, New York, 2001.

[39] J. R. Morison, J. W. Johnson, and S. A. Schaaf. The force exerted by surface waves on piles. Journal of Petroleum Technology, 2(5):149-154, 1950.

[40] A. H. Mousselli. Offshore Pipeline Design, Analysis and methods. PennWell Books, 1981. 
[41] MSC Software Corporation. MSC Marc Volume A: Theory and user information, 2013.

[42] N. M. Newmark. A method of computation for structural dynamics. Journal of the Engineering Mechanics Division, 85(3):67-94, 1959.

[43] J. Nocedal and S. J. Wright. Numerical optimization. Springer, 1999.

[44] B. Nour-Omid and C. C. Rankin. Finite rotation analysis and consistent linearization using projectors. Computer Methods in Applied Mechanics and Engineering, 93(3):353-384, 1991.

[45] R. O'Grady and A. Harte. Localised assessment of pipeline integrity during ultra-deep S-lay installation. Ocean Engineering, 68:27-37, 2013.

[46] Orcina Ltd. OrcaFlex Manual.

[47] C. Pacoste and A. Eriksson. Beam elements in instability problems. Computer Methods in Applied Mechanics and Engineering, 144(1-2):163-197, 1997.

[48] M. Petyt. Introduction to finite element vibration analysis. Cambridge University Press, 2010.

[49] W. J. Pierson and L. Moskowitz. A proposed spectral form for fully developed wind seas based on the similarity theory of S.A. Kitaigorodskii. Journal of Geophysical Research, 69(24):5181-5190, 1964.

[50] C. C. Rankin and F. A. Brogan. An element independent corotational procedure for the treatment of large rotations. Journal of Pressure Vessel Technology, 108(2):165, 1986.

[51] O. Rodrigues. Des lois géometriques qui régissent les déplacements d'un systéme solide dans l'espace, et de la variation des coordonnées provenant de ces déplacements considérés indépendamment des causes qui peuvent les produire. Journal de Mathématiques Pures et Appliquées, 5:380-440, 1840.

[52] T. Sarpkaya. Wave forces on offshore structures. Cambridge University Press, 2010.

[53] J. C. Simo and T. J. R. Hughes. Computational Inelasticity. Springer, New York, 1998.

[54] J. C. Simo and R. L. Taylor. Consistent tangent operators for rateindependent elastoplasticity. Computer Methods in Applied Mechanics and Engineering, 48(1):101-118, 1985.

[55] J. C. Simo and R. L. Taylor. A return mapping algorithm for plane stress elastoplasticity. International Journal for Numerical Methods in Engineering, 22(3):649-670, 1986. 
[56] G. Slee. PLUTO. http://www.combinedops.com/pluto.htm. Accessed on 0503-2019.

[57] C. P. Sparks. The Influence of Tension, Pressure and Weight on Pipe and Riser Deformations and Stresses. Journal of Energy Resources Technology, 106(1):46, 1984.

[58] R. A. Spurrier. Comment on "Singularity-free extraction of a quaternion from a direction-cosine matrix". Journal of Spacecraft and Rockets, 15(4):255, 1978.

[59] E. Stein, R. de Borst, and T. J. R. Hughes. Encyclopedia of computational mechanics. John Wiley \& Sons, 2004.

[60] K. Subbaraj and M. A. Dokainish. A survey of direct time-integration methods in computational structural dynamics-II. Implicit methods. Computers \& Structures, 32(6):1387-1401, Jan 1989.

[61] A. H. van den Boogaard. FeaTure: A finite element analysis toolkit for use in a research environment. https://www.utwente.nl/en/et/ms3/researchchairs/nsm/facilities/software/feature_/. Accessed on 16-08-2017.

[62] M. L. Wilkins. Calculation of elastic-plastic flow. Technical Report UCRL7322, Lawrence Radiation Laboratory, Livermore, 1963.

[63] E. L. Wilson, I. Farhoomand, and K. J. Bathe. Nonlinear dynamic analysis of complex structures. Earthquake Engineering \& Structural Dynamics, 1(3):241-252, 1972.

[64] W. L. Wood, M. Bossak, and O. C. Zienkiewicz. An alpha modification of Newmark's method. International Journal for Numerical Methods in Engineering, 15(10):1562-1566, 1980.

[65] Wood Group Kenny Ltd. PipeLay User Manual, 2016.

[66] M. Yazdchi and M. A. Crisfield. Non-linear dynamic behaviour of flexible marine pipes and risers. International Journal for Numerical Methods in Engineering, 54(9):1265-1308, 2002.

[67] M. S. Zaremba. Le principe des mouvements relatifs et les equations de la mecanique physique. Bulletin international de l'Académie des Sciences de Cracovie, pages 614-621, 1903.

[68] M. S. Zaremba. Sur une forme perfectionée de la théorie de la relaxation. Bulletin international de l'Académie des Sciences de Cracovie, pages 594$614,1903$. 


\section{W}

H.

(1)

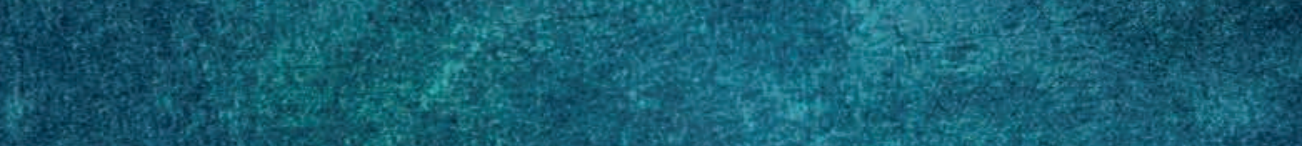

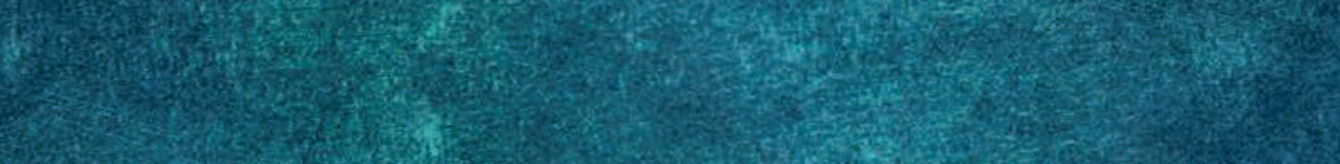
(2) W.

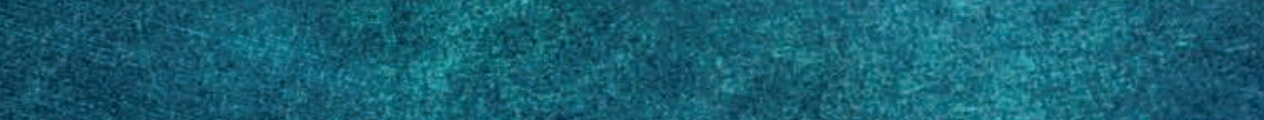
3.

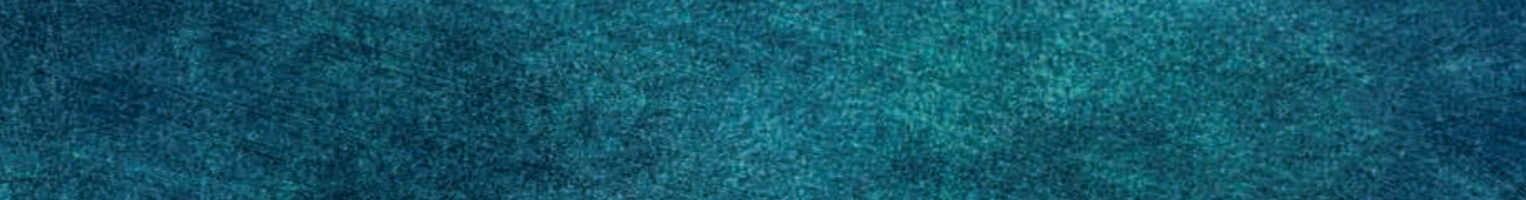
(1)

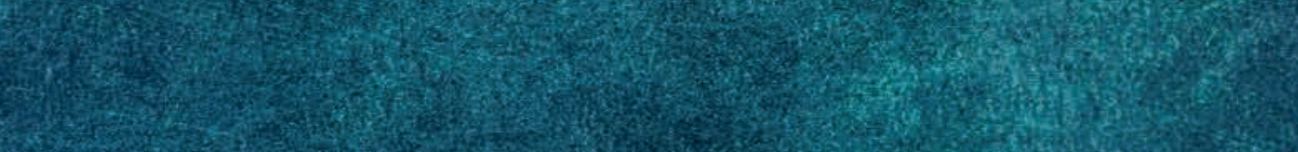

W.1.

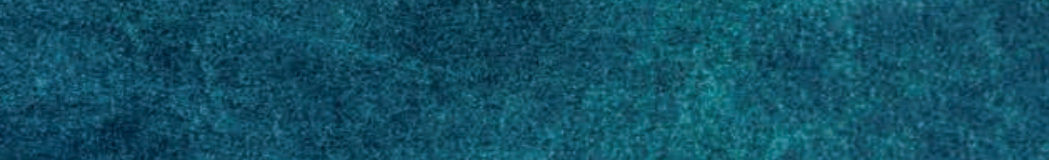
2.7.

3. (3)

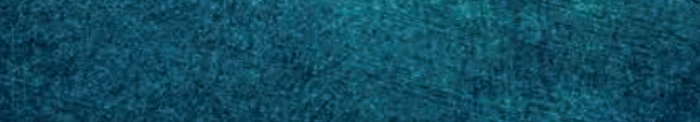



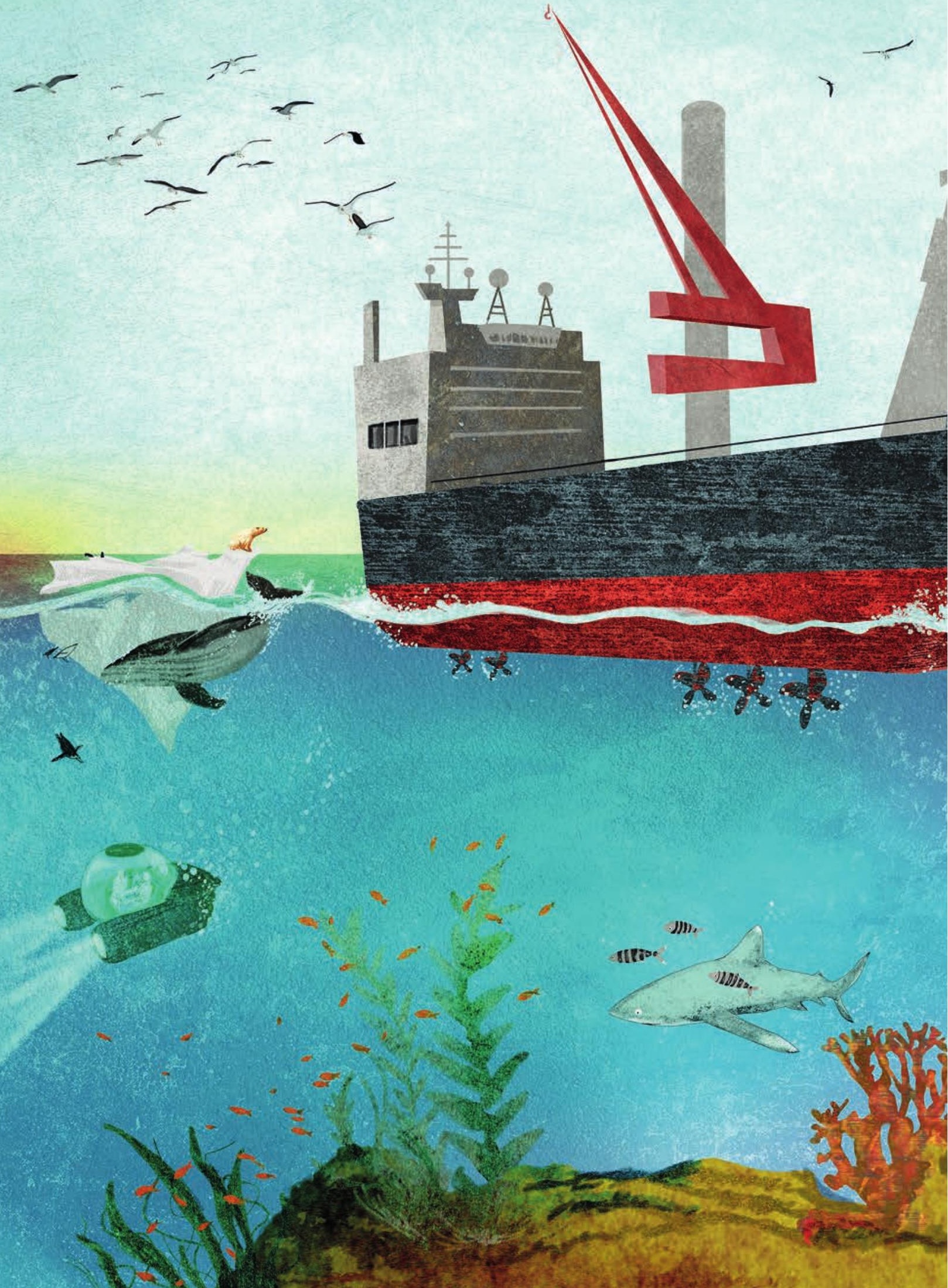